\title{
Pulvinar-cortical interactions for spatial perception and goal-directed actions in non-human primates
}

Dissertation for the award of the degree Doctor rerum naturalium

submitted by

Lydia Gibson

from Ilmenau

\begin{abstract}
Faculty of Biology
Georg-August-Universität Göttingen

Doctoral program Systems Neuroscience

of the Göttingen Graduate School for Neurosciences, Biophysics, and Molecular Biosciences
\end{abstract}

Göttingen, 2018 


\section{Thesis committee}

Dr. Igor Kagan (Supervisor)

Decision and Awareness Group, Cognitive Neuroscience Laboratory, German Primate Center, Göttingen

Prof. Dr. Melanie Wilke

Institute for Cognitive Neurology, University Medical Center, Göttingen

Prof. Dr. Annekathrin Schacht

Institute of Psychology, University of Göttingen, Göttingen

\section{Examination board}

Referee: Dr. Igor Kagan (Supervisor)

Decision and Awareness Group, Cognitive Neuroscience Laboratory, German Primate Center, Göttingen

Co-referee: Prof. Dr. Melanie Wilke

Institute for Cognitive Neurology, University Medical Center, Göttingen

Further members of the examination board:

Prof. Dr. Annekathrin Schacht

Affective Neuroscience and Psychophysiology, University of Göttingen, Göttingen

Dr. Arezoo Pooresmaeili

AG Perception and Cognition, European Neurosciene Institute, Göttingen

PD Dr. Peter Dechent

Institute for Cognitive Neurology, University Medical Center, Göttingen

Prof. Dr. Andrea Antal

Department of Clinical Neurophysiology, Göttingen University Medical School, Göttingen

Date of oral examination: 21st of December, 2017 


\begin{abstract}
The pulvinar nucleus, the largest thalamic nucleus in primates, has been shown to be involved in goal-directed visuomotor behavior related to the contralateral side of visual space. However, there is conflicting evidence on the pulvinar's involvement in either the facilitation of visuomotor responses towards contralateral visual stimuli or the filtering of contralateral visual distractors. Using unilateral electrical microstimulation of the dorsal pulvinar in one male rhesus macaque performing a visuomotor response selection task including choices between visual targets and distractors we found that pulvinar stimulation led to changes in response selection in a time-dependent manner: stimulation starting before the onset of the visual stimuli was associated with a decreased number of saccade choices towards contralateral visual stimuli whereas stimulation starting after stimulus onset led to an increased number of contralateral saccade choices. Both effects, however, may be explained by the pulvinar's involvement in facilitating visuomotor responses towards contralateral stimuli. Moreover, by including eye fixation as an additional response option, we could show that the pulvinar plays a general role in resolving competition between multiple response options. The combination of functional magnetic resonance imaging and microstimulation of the dorsal pulvinar further revealed that the pulvinar plays a functional role in the brain network dedicated to visual attention and visuomotor processing with its effective connectivity strongly overlapping with the connectivity pattern found for the lateral intraparietal area (LIP), a node of the same brain network but located in posterior parietal cortex. However, the magnitude of both pulvinar and LIP stimulation effects on neuronal activity in activated brain regions depended on the current cognitive task and the spatial tuning of the activated areas suggesting that stimulation-induced activity might be modulated depending on the extent of task responsiveness in both the stimulated region and other activated areas. This may result in changes in space representations in the activated brain network which might be neuronal correlates of stimulation-induced changes in visuomotor behavior.
\end{abstract}





\section{Table of contents}

1 General introduction 1

1.1 Anatomical and functional properties of the pulvinar nucleus . . . . . . . 1

1.2 The role of the pulvinar in visuomotor behavior . . . . . . . . . 3

1.3 Aim of the current work . . . . . . . . . . . . . . . 4

2 Effects of pulvinar microstimulation on visuomotor response selection $\quad 7$

2.1 Introduction . . . . . . . . . . . . . . . . . . . . 7

2.2 Methods . . . . . . . . . . . . . . . . . . . . . 14

2.2 .1 Procedures . . . . . . . . . . . . . . . . . 14

2.2.2 Animal preparation . . . . . . . . . . . . . . . . . 14

2.2 .3 MR imaging . . . . . . . . . . . . . . . . . . . 14

2.2.4 Pulvinar targeting . . . . . . . . . . . . . . 15

2.2.5 Electrical microstimulation . . . . . . . . . . . . . . 15

2.2.6 Behavioral paradigm . . . . . . . . . . . . . 16

2.2.7 Distractor color determination . . . . . . . . . . . . . . . 19

2.2 .8 Data analysis . . . . . . . . . . . . . . . . 20

2.3 Results . . . . . . . . . . . . . . . . . . . 23

2.3.1 Response selection . . . . . . . . . . . . . . . 23

2.3.2 Saccade reaction times . . . . . . . . . . . . . 32

2.3 .3 Error rates . . . . . . . . . . . . . . . . 36

2.4 Discussion . . . . . . . . . . . . . . . . . . . . . 39

2.4.1 Pulvinar facilitates visuomotor responses towards contralateral stimuli 39

2.4.2 Pulvinar plays a general role in resolving visuomotor response competition ...................... 41

2.4.3 Pulvinar microstimulation effects on response selection do not only reflect changes in motor execution . . . . . . . . . . . . . 44

2.4.4 Alternative explanations and limitations . . . . . . . . . . . . . 45

2.4 .5 Conclusions . . . . . . . . . . . . . . . . . . 47 
3 Effects of pulvinar and LIP microstimulation on whole-brain space representations $\quad 49$

3.1 Introduction . . . . . . . . . . . . . . . . . . . 49

3.2 Methods ............................ 51

3.2 .1 Procedures . . . . . . . . . . . . . . . 51

3.2 .2 Animal preparation . . . . . . . . . . . . . . 52

3.2.3 Electrical microstimulation . . . . . . . . . . . . . . 52

3.2 .4 MR imaging . . . . . . . . . . . . . . . . . . 53

3.2.5 Pulvinar and LIP targeting . . . . . . . . . . . . . . . . 54

3.2 .6 Behavioral paradigm . . . . . . . . . . . . . . 57

3.2 .7 Data analysis . . . . . . . . . . . . . . . . . 59

3.3 Results . . . . . . . . . . . . . . . . . . . 65

3.3.1 Task performance and eye movements . . . . . . . . . . . 65

3.3.2 BOLD activity induced by $\mathrm{dPul}$ and LIP microstimulation . . . . 75

3.3.3 Effects of dPul and LIP microstimulation on BOLD responses during different cognitive tasks . . . . . . . . . . . . 85

3.3.4 Additive effects of microstimulation on BOLD responses . . . . . . 88

3.3.5 Changes in contraversive selectivity during dPul and LIP microstimulation . . . . . . . . . . . . . . . . 90

3.3.6 Relationship between contraversive selectivity and the magnitude of stimulation effects on BOLD responses . . . . . . . . . . 93

3.4 Discussion . . . . . . . . . . . . . . . . . . . . . . . 114

3.4.1 Effective connectivity of dPul and LIP . . . . . . . . . . . . . . . . 114

3.4.2 Task-dependent effects of microstimulation of dPul and LIP on BOLD activity . . . . . . . . . . . . . . . 117

3.4.3 Effects of microstimulation of $\mathrm{dPul}$ and LIP on spatial tuning . . . 119

3.4.4 Limitations and future directions . . . . . . . . . . . . . . . . 120

3.4 .5 Conclusions . . . . . . . . . . . . . . . . 121

4 General discussion $\quad 123$

$\begin{array}{ll}\text { References } & 127\end{array}$

$\begin{array}{lll}\text { Appendix } & 139\end{array}$ 


\section{Chapter 1}

\section{General introduction}

\subsection{Anatomical and functional properties of the pulvinar nucleus}

The pulvinar nucleus is the largest nucleus of the thalamus in primates and has greatly expanded in size and complexity during mammalian evolution (Harting et al., 1972). It is located posterior, medial, and dorsal to the lateral geniculate nucleus (LGN) covering the superior colliculus (SC) and forming a mass around the brachium of the $\mathrm{SC}$, the axonal tract arising from the SC (Grieve et al., 2000). Anatomically, the pulvinar consists of four major cytoarchitectonic divisions: the inferior pulvinar (PI), the lateral pulvinar (PL), the medial pulvinar (PM), and the anterior or oral pulvinar (PA). PI, PL, and PM have strong anatomical connections to multiple brain regions involved in visuomotor processing and are thus considered visual divisions of the pulvinar (Grieve et al., 2000; Kaas \& Lyon, 2007). PA, by contrast, is most strongly connected to somatosensory brain areas (DarianSmith \& Darian-Smith, 1993; Pons \& Kaas, 1985). However, this traditional anatomical framework often fails to reflect the physiological and connectional properties of the pulvinar subnuclei requiring a more global model of pulvinar connectivity as proposed by Shipp (2003). According to this model, the pulvinar can be divided into two main domains, the dorsal pulvinar (dPul) and the ventral pulvinar (vPul). dPul incorporates most of PM and PA and the dorsal part of PL whereas vPul comprises PI and the ventral part of PL. This model is supported by anatomical studies on the primate pulvinar showing that PI and the ventral part of PL receive their main cortical inputs from early extrastriate visual areas whereas PM and the dorsal part of PL receive inputs from higher association cortices such as parietal cortex (Asanuma et al., 1985; Grieve et al., 2000; Kaas \& Lyon, 2007). Moreover, PM is the only pulvinar subnucleus having strong anatomical connections with areas in frontal lobe (Kievit 
\& Kuypers, 1977) while PI is the subnucleus receiving input from SC (Benevento \& Fallon, 1975; Benevento \& Rezak, 1976; Berman \& Wurtz, 2010; Stepniewska et al., 2000) and the retina (O'Brien et al., 2001). The segregation between a dorsal and a ventral part of PL is further supported by work showing that each part has connectivity that is more similar to the adjacent pulvinar subnucleus (PM or PI, respectively) than to the other part of PL suggesting functional differences between the dorsal and ventral halves of PL (Hardy \& Lynch, 1992). Considering PA as a part of dPul is also anatomically justified since it was shown to be connected to parietal (Acuna et al., 1990; Cappe et al., 2007; Schmahmann \& Pandya, 1990; Yeterian \& Pandya, 1985) and frontal areas (Kuenzle \& Akert, 1977; Morecraft et al., 1992), as well.

dPul and vPul do not only differ in their anatomical networks but also show differences in their functional properties of visual processing. Although neurons in both vPul and dPul respond to visual stimuli (Petersen et al., 1985; Robinson et al., 1986), the number of visually responsive cells is higher in vPul than in dPul (Petersen et al., 1985; Robinson et al., 1986). In addition, vPul is retinotopically organized (Bender, 1981; Petersen et al., 1985; Shipp, 2003) and vPul neurons have well-defined visual receptive fields ranging from $1^{\circ}$ to $5^{\circ}$ in diameter and confined to the contralateral hemifield. vPul cells also show sensitivity to basic characteristics of visual stimuli in the receptive field such as orientation or movement direction (Bender, 1982; Petersen et al., 1985). By contrast, dPul shows a poor retinotopic arrangement (Benevento \& Miller, 1981; Petersen et al., 1985) with neurons having large receptive fields (Petersen et al., 1987) ranging from $10^{\circ}$ to $60^{\circ}$ in diameter and located entirely in the contralateral or the ipsilateral hemifield or even spanning both hemifields. Although some dPul neurons have the centers of their receptive fields on the vertical meridian or well within the ipsilateral hemifield, the centers are usually located in the contralateral hemifield (Benevento \& Miller, 1981) and visual responses to contralateral visual stimuli are stronger than responses to ipsilateral stimuli (Dominguez-Vargas et al., 2017). In addition, dPul neurons show less (Benevento \& Miller, 1981) and only very crude selectivity for basic properties of visual stimuli (Petersen et al., 1987). Instead, dPul cells can be selective for more complex features of visual stimuli such as color (Benevento \& Port, 1995) and they exhibit enhanced responses to complex visual stimuli representing faces or snakes (Van Le et al., 2013). Both vPul and dPul neurons discharge during and after saccadic eye movements towards the contralateral hemifield (Acuna et al., 1983; Dominguez-Vargas et al., 2017; Petersen et al., 1987; Robinson et al., 1986). Neuronal signals in vPul have also been found to be related to saccadic suppression (Berman et al., 2016). Furthermore, attentional modulation of neuronal responses has been found in both domains of the pulvinar (Bender \& Youakim, 2001; Petersen et al., 1985, 1987; Saalmann et al., 2012; Zhou et al., 2016). 


\subsection{The role of the pulvinar in visuomotor behavior}

The functions of the primate pulvinar in visuomotor behavior have been studied using multiple causal interference techniques such as lesioning (Bender \& Baizer, 1990; Bender $\&$ Butter, 1987; Chalupa et al., 1976; Leiby III et al., 1982), pharmacological perturbation (Petersen et al., 1987; Wilke et al., 2013, 2010), and electrical microstimulation (Blum, 1984, 1985; Dominguez-Vargas et al., 2017; Vanni et al., 2015). Moreover, the pulvinar's role in visuomotor processing and its underlying neuronal correlates have been studied on patients with pulvinar lesions following stroke (Michael \& Buron, 2005; Rafal \& Posner, 1987; Snow et al., 2009; Van der Stigchel et al., 2010; Ward \& Danziger, 2005) and healthy human subjects using different non-invasive imaging techniques (Fischer \& Whitney, 2012; LaBerge $\&$ Buchsbaum, 1990; Strumpf et al., 2013; Villeneuve et al., 2005).

Pulvinar lesion studies on non-human primates showed that both $\mathrm{dPul}$ and vPul do not seem to play a critical role in basic visuomotor behavior such as the detection of brief visual stimuli (Leiby III et al., 1982), visual search (Bender \& Butter, 1987), or size constancy (Ungerleider et al., 1977). Instead, vPul seems to relay retinal information to cortical areas of the dorsal visual stream supporting visually-guided behavior early in life (Bourne \& Morrone, 2017). Moreover, pharmacological inactivation of the ventral part of PL can almost extinguish visual responses of neurons in the primary visual cortex (Purushothaman et al., 2012) and leads to an increase or decrease of spontaneous activity and visual responses of V2 neurons including changes in orientation and/or direction selectivity (Soares et al., 2004). At the behavioral level, ventrolateral pulvinar inactivation causes severe impairment in an attentional saccade task confined to the visual hemifield contralateral to the side of injection reflecting either sensory deficits or even profound neglect (Zhou et al., 2016). Similarly, inactivation of posterior PL leads to impaired performance in an attentional color discrimination task when a target is shown in the affected hemifield together with a distractor in the intact hemifield. However, performance was not affected when no distractor was presented or when both target and distractor are shown in the affected hemifield suggesting that PL plays a critical role in attention when stimuli in opposite hemifields compete for attentional resources (Desimone et al., 1990). Pharmacological inactivation or activation of the macaque $\mathrm{dPul}$ results in impaired or facilitated shifting of visual attention towards the side of visual space that lies contralateral to the side of perturbation, respectively (Petersen et al., 1987). Furthermore, dPul inactivation leads to a bias in saccade target selection and spontaneous visual exploration against the hemifield contralateral to the side of inactivation resembling signs of visual extinction (Wilke et al., 2010). Interestingly, this bias in saccade target selection can be diminished by higher visual salience, i.e. higher luminance, of the 
stimulus presented in the affected hemifield. However, a higher reward associated with that stimulus is even more effective in alleviating this target selection bias (Wilke et al., 2013).

In humans, pulvinar lesions can cause deficits in stereoacuity (Takayama et al., 1994) and slowed reactions to visual targets presented in the affected hemifield (Rafal \& Posner, 1987). The results of a series of selective attention studies on a group of patients with pulvinar lesions suggest that dPul is involved in the activation of visuomotor responses towards visual stimuli in the contralateral hemifield, especially under conditions of competition between responses related to stimuli presented in opposite hemifields, and in binding a stimulus representation to a corresponding visuomotor response (Ward \& Danziger, 2005). A study using electrical microstimulation of dPul in monkeys supports these conclusions (Dominguez-Vargas et al., 2017). In contrast, unilateral damage in the human vPul leads to impairments in the spatial localization and the binding of visual features to coherent representations of visual objects in the visual field contralateral to the lesion in a spatiotopic manner (Ward et al., 2002). Another study on a patient with a complete loss of the left pulvinar showed that pulvinar is also involved in the rapid processing of threatening visual stimuli (Ward et al., 2005). Studies on healthy human subjects further suggest that the pulvinar encodes attended objects with high precision while ignored objects are barely represented (Fischer \& Whitney, 2012) and that the pulvinar is involved in higher-order motion processing (Villeneuve et al., 2005).

\subsection{Aim of the current work}

In summary, previous work on the macaque and human pulvinar showed that pulvinar perturbation affects visuomotor behavior related to the contralateral side of space. However, there is an ongoing debate about the cognitive mechanisms underlying these changes in behavior, especially when visual stimuli in opposite hemifields signal competing visuomotor responses. The first chapter of this $\mathrm{PhD}$ thesis presents a behavioral study conducted on one male rhesus monkey showing evidence that dPul is involved in the facilitation of visuomotor responses towards visual stimuli in the contralateral hemifield contributing to the scientific discourse on the pulvinar's cognitive function in visuomotor behavior. Furthermore, anatomical studies have revealed the diverse anatomical connectivity of both dPul and vPul. However, the functional connectivity of $\mathrm{dPul}$ and vPul and the pulvinar's role in the frontotemporo-parietal cortical network involved in visual attention and visuomotor behavior is still unknown. Moreover, the neuronal mechanisms underlying changes in visuomotor behavior due to pulvinar perturbation are not understood. The second chapter of this $\mathrm{PhD}$ thesis presents an fMRI study on two male rhesus monkeys performing a fixation and a memory-guided saccade task with and without electrical microstimulation of dPul or the 
lateral intraparietal area (LIP), an important hub of the fronto-temporo-parietal network sharing strong anatomical connections with $\mathrm{dPul}$. This technique allowed for the direct comparison of the functional connectivity of dPul and LIP identifying dPul as part of the attention and saccade brain network. In addition, the superior temporal sulcus (STS) and the dorsolateral prefrontal cortex (dlPFC) were identified as the common nodes of the functional networks of dPul and LIP. Importantly, the effects of electrical microstimulation of both $\mathrm{dPul}$ and LIP on brain activity were found to be dependent on the current visuomotor task providing insight into the neuronal mechanisms related to behavioral changes induced by pulvinar perturbation. 



\section{Chapter 2}

\section{Effects of pulvinar microstimulation on visuomotor response selection}

\subsection{Introduction}

Visual scenes often contain multiple spatial locations that can be selected for gaze redirection and thus serve as potential targets for saccades. The pulvinar's role in visual target selection has been under debate considering two opposing functions the pulvinar may perform. LaBerge \& Buchsbaum (1990) investigated the pulvinar's role in visual distractor processing using positron emission tomography. In this study healthy subjects were either shown a target letter surrounded by eight other letters (filtering task) or only one larger target letter (nonfiltering task) presented in one hemifield, respectively. Subjects were to respond via button press only when the target letter matched the letter "O". Greater activity was found in the pulvinar contralateral to the display of the filtering task than in the pulvinar that was contralateral to the display of the nonfiltering task. The authors concluded that the identification of an object in a cluttered visual scene may involve the pulvinar as a filtering mechanism operating on the information flow between cortical areas involved in early feature processing and cortical areas responsible for object recognition. The hypothesis that the pulvinar filters distracting visuospatial signals was further supported by findings of Van der Stigchel et al. (2010) who had human patients with a unilateral pulvinar lesion perform an oculomotor task that required subjects to inhibit reflexive saccadic eye movements towards a visual distractor with abrupt onset in order to make a correct saccade to the target stimulus. In this oculomotor capture paradigm the interference of the distractor was stronger when it was presented contralateral to the side of the lesion (contralesional) compared to when it was presented in the ipsilesional visual field, as reflected in a higher number of erroneous reflexive 
saccades. These results may be explained by an impaired ability to filter visual distractors presented in the contralesional hemifield following pulvinar lesions. Furthermore, in an fMRI study using a visual search paradigm in healthy human subjects Strumpf et al. (2013) identified distractor filtering as the main attentional operation subserved by the pulvinar.

However, there is evidence supporting the hypothesis that the pulvinar's function may be the facilitation of visuomotor responses towards potential targets on the contralateral side of visual space rather than active filtering of contralateral distractors. Ward \& Danziger (2005) reviewed multiple behavioral studies on human patients with unilateral pulvinar lesions performing an adapted version of the flanker task developed by Eriksen \& Eriksen (1974). In this adapted paradigm only a single flanker is presented either contralesional or ipsilesional to a visual target that appears at a known location. Patients are required to report the color of the target stimulus by vocal report or by pressing a button with the ipsilesional hand while ignoring the irrelevant flanker, which can be congruent (same color as the target) and thus indicating the same response as the target stimulus or incongruent (different color than the target), i.e. indicating a response different from that indicated by the target stimulus (Cohen et al., 1995; Rafal et al., 1996; Ro et al., 1998). Consistent across studies, ipsilesional distractors led to greater interference than contralesional distractors, as reflected in slower reactions to contralesional targets presented with ipsilesional flankers (Ward \& Danziger, 2005). In contrast to Rafal \& Posner (1987) who found substantial slowing of reactions to contralesional targets in thalamic stroke patients performing a spatial attention task, when averaging across conditions with congruent and incongruent flankers patients with pulvinar damage showed equivalent reaction times to targets in both hemifields arguing against a general slowing of contralesional processing (Danziger et al., 2002). Interestingly, neutral flankers that did not interfere with the response indicated by the target stimulus barely affected reaction times suggesting that pulvinar damage only impairs the activation of responses to contralesional targets under conditions of response competition, that is, when a response to a contralesional object must be activated in preference to a conflicting response indicated by an ipsilesional object (Danziger et al., 2004). Moreover, when asked to make a saccade to the visual target that appeared first in a temporal-order judgment task pulvinar patients exhibit a bias against making saccades towards contralesional targets although the perceptual decision may have been correct. Similarly, when performing an antisaccade task that requires subjects to make a saccade to the hemifield opposite to the location of the target stimulus pulvinar patients show longer latencies to initiate antisaccades away from contralesional targets compared to ipsilesional targets (Arend et al., 2008) suggesting an impaired activation of saccadic responses indicated by contralesional visual targets. These results are also in line 
with Ward \& Danziger's (2005) general conclusion that the pulvinar does not filter distractors but is involved in contralateral response selection and activation.

Several animal studies investigated the role of the pulvinar in visuomotor behavior using causal interference techniques. First, reversible, unilateral pharmacological inactivation of the macaque dPul was found to bias spontaneous, exploratory eye movements as well as saccade choices in favor of responses towards the ipsilesional side of space. In addition, although pulvinar inactivation did not affect the execution of saccades to single contralesional targets, saccades to ipsilesional targets were faster suggesting that the dorsal aspect of the pulvinar may play a critical role in spatial attention and visual target selection (Wilke et al., 2013, 2010). Moreover, similar to the paradigm used in studies on pulvinar patients (Ward \& Danziger, 2005), Desimone et al. (1990) had monkeys perform a color-discrimination task with a spatial attentional cue with simultaneous unilateral, pharmacological inactivation of the PL using muscimol, a GABA agonist. First, a briefly flashed spatial cue was presented in the left or right hemifield. In distractor trials this cue was followed by two briefly presented colored bars. By definition, the target stimulus was the bar that appeared at the cued location. The animal had to indicate the target's color with a lever press, ignoring the distractor. The distractor could either have the same or a different color than the target. As a control, monkeys performed the same task without distractors. In this control task pulvinar inactivation only slightly impaired color discrimination. However, similar to what Ward \& Danziger (2005) found in pulvinar patients, when a contralesional target was presented with an ipsilesional distractor the animal was severely impaired as reflected in a higher number of color discrimination errors whereas an improvement in performance was observed in the opposite condition with an ipsilesional target and a contralesional distractor. Interestingly, when both target and distractor were placed in the same visual field there was very little effect of pulvinar inactivation on color discrimination. These findings suggest that the pulvinar plays a critical role when attention is directed to a visual target in the presence of a distractor in the opposite hemifield, i.e. only under conditions of response competition between the two hemifields. Petersen et al. (1987) investigated the effects of unilateral reversible pharmacological manipulation of the activity of the dorsomedial part of the PL on performance in a different visuospatial cueing task in macaque monkeys. In this task a spatial cue presented either in the left or the right hemifield was followed by a peripheral target stimulus which could either appear on the same side as the cue (valid condition) or on the opposite side (invalid condition). The animals were to fixate a central fixation spot and press a bar as soon as the target stimulus appeared on the screen. While the animals were peforming the task the pulvinar was either inactivated by injection of the GABA agonist muscimol or activated by injecting bicuculline, a GABA antagonist. In valid trials when cue 
and target were presented in the hemifield ipsilateral to the injection site, neither pulvinar inactivation nor activation had an effect on the animals' reaction times. In contrast, when both cue and target were presented contralateral to the injection site pulvinar inactivation led to a slowing of reaction times whereas activation of the pulvinar slightly shortened response times. Similarly, in invalid trials responses to contralateral targets preceded by ipsilateral cues were slowed after pulvinar inactivation whereas pulvinar activation led to faster responses. However, in invalid trials with ipsilateral targets preceded by contralateral cues the effects of injection were reversed. In this condition, pulvinar inactivation resulted in faster responses to the target stimulus and pulvinar activation was associated with slower reaction times. Taken together, these results suggest that the pulvinar is involved in facilitating shifts of visuospatial attention towards the contralateral visual field.

However, the findings of the studies described above also provide evidence for the pulvinar being involved in the activation of visuomotor responses towards contralateral visual stimuli rather than filtering contralateral distractors and may resemble the findings of the human patient studies mentioned above. In Wilke et al. (2010) when monkeys were free to choose between two visual targets presented in opposite hemifields dPul inactivation may have diminished the visuomotor response activated by contralesional targets leading to a relatively stronger response activation for ipsilesional targets and thus an ipsilesional bias in saccade choices. In Desimone et al.'s (1990) study pulvinar inactivation impaired color discrimination for contralesional targets presented with an ipsilesional distractor. In this condition contralesional targets may activate a weaker visuomotor response than under control conditions resulting in a relatively stronger response activated by the ipsilesional distractor. This may result in a higher number of incorrect responses towards ipsilesional distractors. Conversely, when presenting an ipsilesional target together with a contralesional distractor the animals' performance improved. Again, this may be explained by a weaker response activated by the contralesional stimulus, in this condition the distractor, making the distractor less effective in interfering with the processing of the ipsilesional stimulus, in this case the target. Similarly, in Petersen et al.'s (1987) study unilateral pulvinar inactivation led to a general slowing of responses to targets presented contralateral to the side of inactivation but it also diminished the effectiveness of contralateral cues in automatic shifting of attention as indicated by faster responses in invalid trials with contralateral cues and ipsilateral targets. In this condition, the response activated by the contralateral cue might be weaker after pulvinar inactivation leading to a relatively stronger response activation by the ipsilateral target and hence faster reaction times. Conversely, pulvinar activation generally speeded up responses to contralateral targets and slowed responses to ipsilateral targets preceded by contralateral cues suggesting that pulvinar activation might have an opposite effect. In particular, after 
pulvinar activation contralateral targets may activate a stronger response than in control sessions generally speeding up reactions to contralateral targets. Similarly, contralateral cues might also activate a stronger response and the animal may have to counteract the response activated by the contralateral cue before being able to respond to the ipsilateral target stimulus resulting in slower reaction times.

This interpretation is further supported by recent findings obtained with electrical microstimulation of the $\mathrm{dPul}$ in macaque monkeys performing a two-alternative forced choice saccade task (Dominguez-Vargas et al., 2017). In this task monkeys were either instructed to make a saccade to a peripheral stimulus presented in the left or the right hemifield or the animals were presented with two peripheral stimuli in opposite hemifields. In the latter condition, animals could freely choose either one of the stimuli as the saccade target. Unilateral electrical microstimulation was applied to the dPul either before or after visual target onset. Stimulation starting before target onset shortened reaction times to ipsiversive (ipsilateral to the side of stimulation) targets whereas stimulation starting at or after target onset delayed saccades to both ipsiversive and contraversive (contralateral to the side of stimulation) targets. Moreover, in saccade choice trials dPul stimulation starting before target onset led to an increased proportion of ipsiversive target choices whereas stimulation at or after target onset was associated with a higher number of contraversive choices. The authors concluded that dPul stimulation may induce a contraversive drive, i.e. activation of visuomotor responses to the side contralateral to the side of stimulation which has to be counteracted by the animals when being stimulated before target onset as in this stage of the task the animals were still required to maintain central eye fixation. This ipsiversive compensatory mechanism may be engaged until the motor planning or execution stage, i.e. after target onset, resulting in the observed increase in ipsiversive choices for stimulation starting before target onset. However, when stimulation is applied at or after target onset the stimulation-induced contraversive drive facilitates responses to contraversive visual targets leading to a higher proportion of contraversive target choices. Interestingly, in a memory-guided saccade task where the visual presentation of the targets and movement execution are temporally separated from each other pulvinar microstimulation did not affect saccadic choice behavior indicating that the pulvinar mostly influences spatial choices when visual stimulus and action are temporally close to each other. This conclusion is in line with evidence provided by pulvinar patient studies suggesting that the pulvinar may play a critical role in integrating visual information with action systems by enabling direct linkages between stimuli and actions (Arend et al., 2008).

However, the study by Dominguez-Vargas et al. (2017) - which, to our knowledge, is the only study available investigating the role of pulvinar in visual target selection using electrical microstimulation - did not address the question whether the pulvinar is responsible 
for contralateral response activation or filtering of contralateral distracting visual information as the two-alternative forced choice paradigm did not include conditions with visual distractors that would engage a filtering mechanism. Moreover, previous research has shown that the behavioral relevance of target choices such as a higher reward outcome for one of two potential saccade options can modulate the effects of pulvinar inactivation on saccade choices (Wilke et al., 2013). In the free-choice task used by Dominguez-Vargas et al. (2017), however, both saccade options were associated with equal outcomes indicating little behavioral relevance of the choice. In the present study we used unilateral electrical microstimulation of the dPul to investigate whether the pulvinar's main function is the facilitation of visuomotor responses to contralateral visual targets or the filtering of contralateral visual distractors using a forced-choice saccade task including choices between correct targets and incorrect distractors associated with different behavioral outcomes (reward vs. no reward). More specifically, similar to Dominguez-Vargas et al. (2017), we electrically stimulated the dPul before, at, or after the onset of one or two visual stimuli presented in one or in opposite hemifields. In all conditions, the animal had to identify the target stimulus, if present, and make a saccade to that stimulus. When two targets were presented the animal could make a saccade to either one of them whereas the animal was to maintain central eye fixation when only one or two distractors were presented.

We view the two potential mechanisms of pulvinar function as two opposing mechanisms as they lead to different behavioral predictions in our paradigm, especially when stimulation starts at or after stimulus onset (see Fig. 2.1). If the pulvinar's main function was a general facilitation of visuomotor responses to contralateral stimuli, one would expect unilateral microstimulation to increase the proportion of saccade choices towards contraversive stimuli including incorrect responses towards contraversive distractors accompanied by a decrease in ipsiversive choices. In contrast, if the pulvinar mainly serves to filter visual distractors, unilateral microstimulation should lead to a stronger filtering of contraversive distractors resulting in a decrease in incorrect choices towards contraversive distractors and a higher proportion of ipsiversive choices. However, if the pulvinar's major role was the filtering of visual distractors, it remains unclear how pulvinar microstimulation would affect choices between two equally rewarded saccade options or when a target stimulus is presented in the contraversive hemifield. In these cases stimulation might not have an effect on response selection at all as there is no information present in the contraversive hemifield that needs to be filtered out. On the other hand, stimulation might still activate the filtering mechanism resulting in an erroneous filtering of contraversive targets and thus a decreased number of saccades towards these targets. 


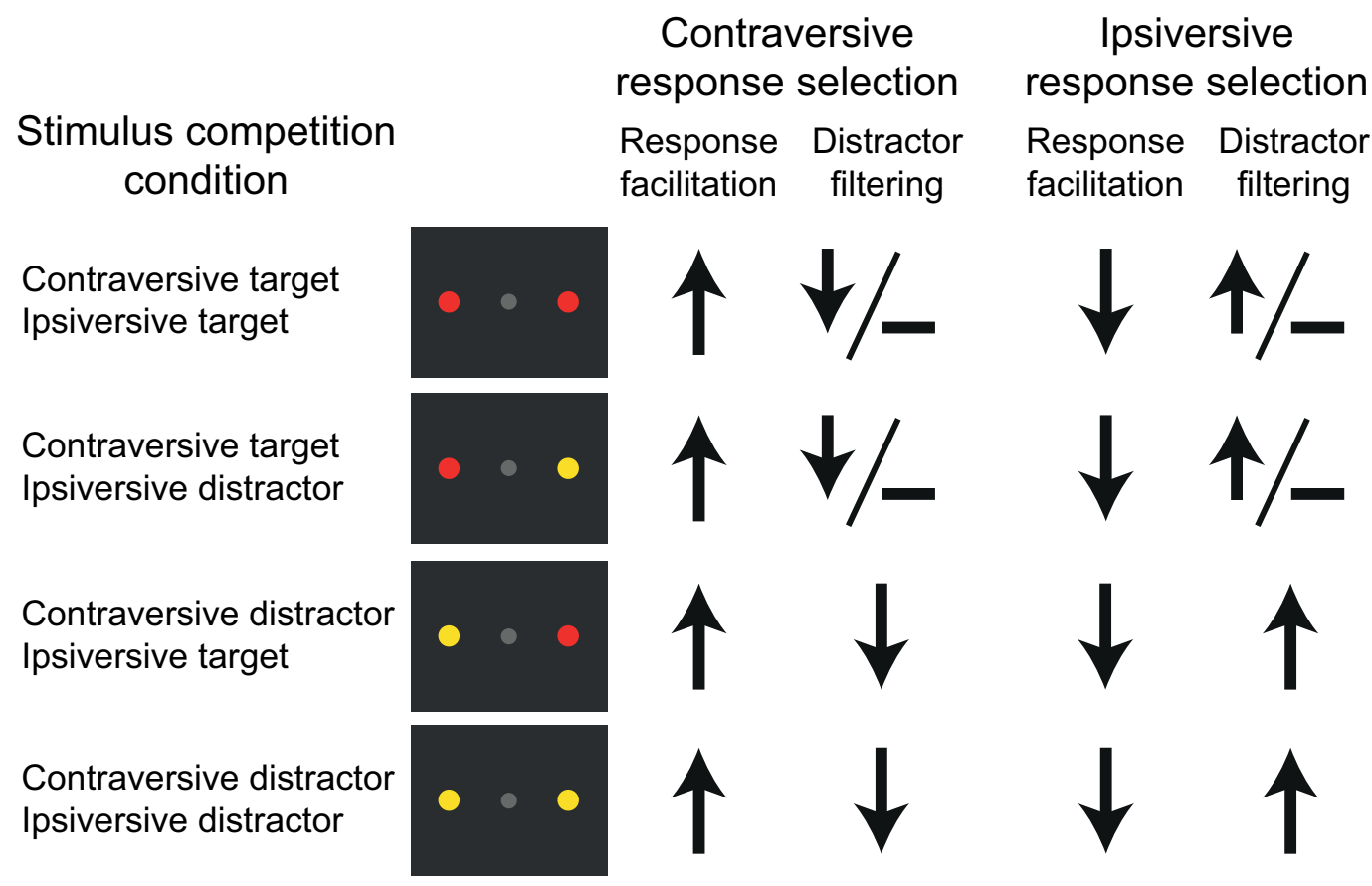

Fig. 2.1 Predicted effects of unilateral dPul stimulation after stimulus onset on contraversive and ipsiversive response selection for stimulus conditions with competition between two potential saccade options. Note the opposing expected effects on response selection for the two potential functions of the pulvinar, contraversive response facilitation ("Response facilitation") vs. contraversive distractor filtering ("Distractor filtering"), in stimulus conditions with a contraversive distractor. In conditions with a contraversive target pulvinar stimulation might either have similar effects as in conditions with a contraversive distractor or stimulation might have no effect on response selection. Red dots: targets, yellow dots: distractors, grey dots: fixation spot. Contraversive: contralateral to the side of stimulation (left), ipsiversive: ipsilateral to the side of stimulation (right), upward arrows: expected increase in response selection, downward arrows: expected decrease in response selection, hyphen: no expected effect on response selection.

As mentioned above, previous research suggests that causal manipulation of pulvinar activity only affects visuomotor behavior under conditions of response competition between the two hemifields (Danziger et al., 2004; Desimone et al., 1990). Importantly, in all studies mentioned above there was only response competition between peripheral visual stimuli indicating a certain action. In contrast, in the present study maintaining central eye fixation instead of making a saccade to a peripheral stimulus always served as an alternative response option introducing response competition between potential peripheral saccade targets and the fixation spot, i.e. withholding a saccade response. This allowed us to further investigate whether the pulvinar is most strongly involved in resolving competition between peripheral stimuli linked to a certain action or whether the pulvinar plays a more general role in 
resolving competition between different potential visuomotor response options including a non-saccadic response. Moreover, we used two levels of perceptual difficulty with distractors that were either easily distinguishable from target stimuli or perceptually similar to targets to further study if perceptual similarity between saccade targets and distractors is a prerequisite to drive response competition between potential saccade targets.

\subsection{Methods}

\subsubsection{Procedures}

All experimental procedures were conducted in accordance with the European Directive 2010/63/EU, the corresponding German law governing animal welfare, and German Primate Center institutional guidelines. The procedures were approved by the responsible government agency (Niedersaechsisches Landesamt fuer Verbraucherschutz und Lebensmittelsicherheit (LAVES), Oldenburg, Germany).

\subsubsection{Animal preparation}

One male rhesus monkey (Macaca mulatta), weighing $9 \mathrm{~kg}$ served as a subject. In an initial surgery, the animal was implanted with an MRI-compatible polyetheretherketone (PEEK) head post embedded in a bone cement head cap (Palacos with gentamicin; BioMet) anchored by ceramic screws (Rogue Research) under general anesthesia and aseptic conditions. MRvisible markers were embedded in the head cap to aid the planning of the chamber in stereotaxic space (right hemisphere: center at $0.5 \mathrm{~A} / 14.5 \mathrm{~L} \mathrm{~mm}$, tilted $-11 \mathrm{P} / 27 \mathrm{~L}$ degrees) with the MR-guided stereotaxic navigation software Planner (Ohayon \& Tsao, 2012). A separate surgery was performed to implant a PEEK MRI-compatible chamber (inside diameter $22 \mathrm{~mm}$ ) allowing access to the right pulvinar. After confirming chamber positioning with a postsurgical MRI, a partial craniotomy was made inside the chamber.

\subsubsection{MR imaging}

The monkey was scanned in a 3 T MRI scanner (Magnetom TIM Trio; Siemens). Full-head T1-weighted (3D magnetization-prepared rapid gradient-echo, MPRAGE, 0.5mm isometric) and additional T2-weighted (rapid acquisition with relaxation enhancement, RARE, 0.25 $\mathrm{mm}$ in plane, $1 \mathrm{~mm}$ slice thickness) images with the slice package aligned to the chamber vertical axis were acquired before and after chamber implantation in an awake state using the built-in gradient body transmit coil and a custom single-loop receive coil (Windmiller Kolster 
Scientific). T1- and T2-weighted scans were coregistered and transformed into "chamber normal" orientation (aligned to the chamber vertical axis) and into AC-PC space for electrode targeting and visualization. These images were acquired with the chamber and the grid filled with gadolinium (Magnevist; Bayer)/saline solution (proportion 1:200) with tungsten rods inserted in predefined grid locations for alignment purposes.

\subsubsection{Pulvinar targeting}

For every stimulation site the electrode location was estimated based on anatomical MRI. A custom-made MR-compatible polyetherimide (Ultem) grid (0.8 $\mathrm{mm}$ hole spacing, 0.45 $\mathrm{mm}$ hole diameter) and a custom-made plastic XYZ manipulator drive (design courtesy of Dr. Sebastian Moeller (Moeller et al., 2008)) were used to position platinum-iridium electrodes (FHC, see section 3.2.3 for detailed specifications) in the corresponding grid hole and estimated depth. Grid hole determination was based on anatomical MRI using Planner (Ohayon \& Tsao, 2012) and BrainVoyager (Version 2.4.2.2070, 64-bit; Brain Innovation $\mathrm{BV})$. During penetration, the electrode was protected by a custom-made stainless steel guide tube (450 $\mu \mathrm{m}$ outer diameter, 27 gauge Spinocan, Braun Melsungen). A stopper (530 $\mu \mathrm{m}$ inner diameter, $665 \mu \mathrm{m}$ outer diameter, 23 gauge MicroFil; World Precision Instruments) ensured that the guide tube only penetrated the dura and minimally the cortex below. Before penetration, the electrode tip was aligned to the guide tube tip and was held in place by a drop of melted petroleum jelly.

We stimulated two different sites in the right $\mathrm{dPul}$, twelve sessions at a more anterior site and four sessions at a more posterior site. For localization of dPul stimulation sites we used the traditional segregation of the pulvinar nucleus into PM, PL, and PI as used in currently online available and downloadable atlases (Calabrese et al., 2015; Rohlfing et al., 2012). As shown in Fig. 2.2, the stimulation sites corresponded mostly to the PM and the dorsal part of the PL. The brachium of the superior colliculus (bsc) and other neighboring structures such as the reticular thalamic nucleus and the tail of the caudate nucleus were avoided.

\subsubsection{Electrical microstimulation}

An S88X dual output square pulse stimulator (Grass Products, Natus Neurology, USA) triggered by a MATLAB-based task controller generated $200 \mathrm{~ms}$ trains of twin pulses at $300 \mathrm{~Hz}$, which in turn triggered a constant current stimulus isolator A365 (World Precision Instruments, USA) to produce 60 biphasic pulses. The current (200-250 $\mu \mathrm{A})$ was delivered to the target structure using single monopolar electrodes (platinum-iridium, $100 \mathrm{~mm}$ length, $125 \mu \mathrm{m}$ thick core, initial $2 \mathrm{~cm}$ glass coating with an exposed tip of $40 \mu \mathrm{m}$, total thickness 
of $230 \mu \mathrm{m}$ including polyamide tubing coating, customer part ID: UEIK1, FHC Inc., USA). A return (reference) tungsten rod was placed in the chamber filled with saline. Voltage drop at a $10 \mathrm{k} \Omega$ resistor in series with the electrode was monitored using a 4 channel $1 \mathrm{GS} / \mathrm{s}$ Tektronix TDS2004C oscilloscope. The manufacturer-specified impedance of the electrodes was $300-336 \mathrm{k} \Omega$. The initial impedance measured at $1000 \mathrm{~Hz}$ before the experiment was $200-650 \mathrm{k} \Omega$. Since the impedance dropped dramatically after a few stimulation trains were applied, before each session 20-30 pulse trains were delivered to the electrode immersed in saline using $250 \mu \mathrm{A}$ current, in order to bring the electrode impedance to a more stable regime. Following this procedure, the impedance ranged from $20 \mathrm{k} \Omega$ to $70 \mathrm{k} \Omega$.

\subsubsection{Behavioral paradigm}

The monkey was sitting in a dark room in a custom-made primate chair with the head restrained $30 \mathrm{~cm}$ away from a 27" LED display ( $60 \mathrm{~Hz}$ refresh rate, model HN274H, Acer Inc. USA). The gaze position of the right eye was monitored at $220 \mathrm{~Hz}$ using an MCU02 ViewPoint infrared eyetracker (Arrington Research Inc. USA). The monkey's face and body

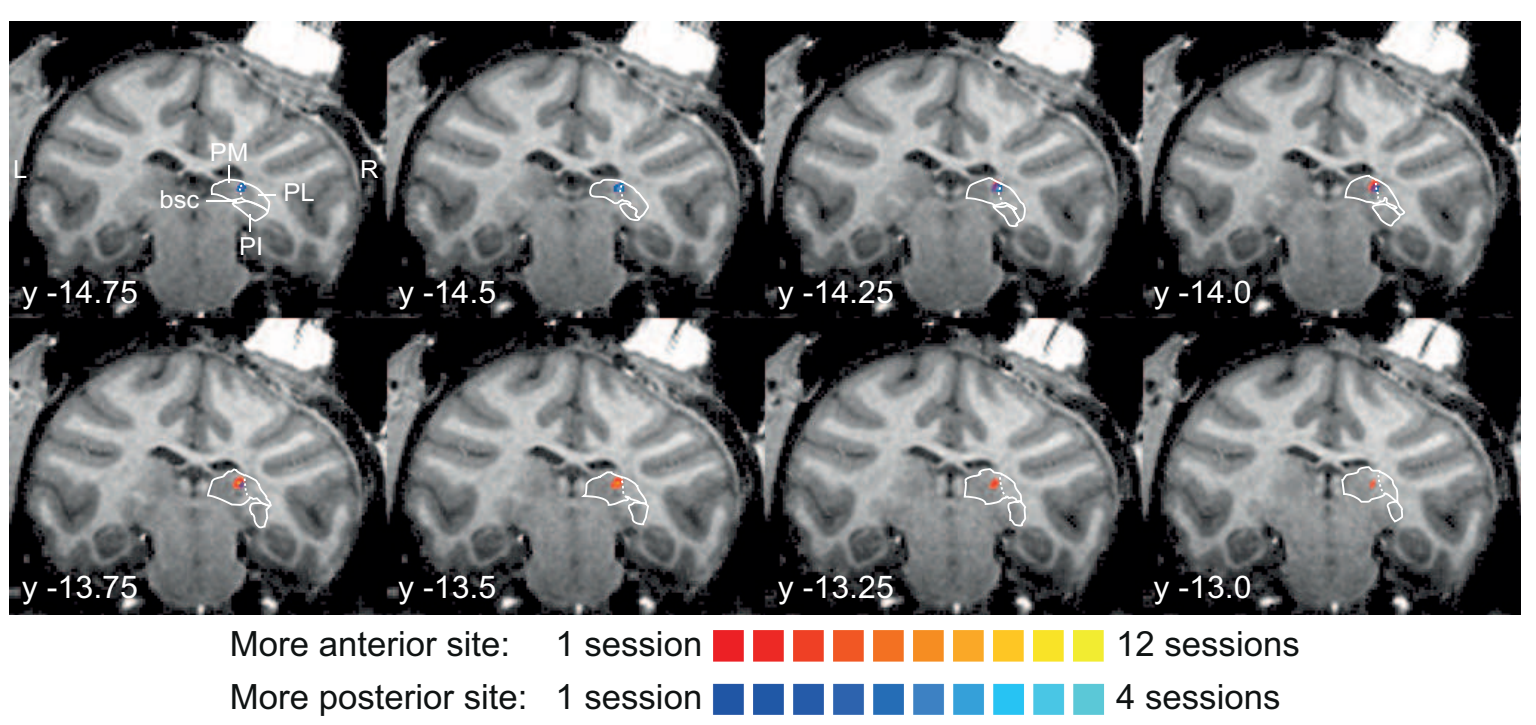

Fig. 2.2 Probability map of electrode positions across sessions projected onto a highresolution MR image of the animal's brain in AC-PC space. Pulvinar nucleus outlines (PM, PL, PI) were adapted from the NeuroMaps atlas (Rohlfing et al., 2012). Red/yellow map: overlap of the estimated electrode positions between 12 sessions with stimulation at a more anterior dPul stimulation site. Blue map: overlap of the estimated electrode positions between 4 sessions with stimulation at a more posterior dPul stimulation site. Y: distance from AC-PC origin in the anterior/posterior plane in millimeters, L: left, R: right, PM: medial pulvinar, PL: lateral pulvinar, PI: inferior pulvinar, bsc: brachium of the superior colliculus. 
were monitored with infrared cameras to ensure that microstimulation did not elicit abrupt movements or signs of discomfort. A MATLAB-based task controller (version R2012b, The MathWorks, Inc., USA) and the Psychophysics Toolbox (Brainard, 1997) were used to control stimulus presentation.

\section{Fixation task}

At the beginning of each stimulation session the monkey performed an eye fixation task in order to determine the optimal current strength and electrode position for subthreshold microstimulation that did not evoke saccades. To this end, we first placed the electrode at the estimated location in the center of the dPul. Then, in several blocks consisting of 20 trials each the animal was presented with a dark grey central fixation spot $\left(0.5^{\circ}\right.$ diameter $)$ which turned light grey as soon as the animal acquired eye fixation. The monkey was required to maintain eye fixation for $2000 \mathrm{~ms}$ within a radial window of $5^{\circ}$ around the fixation spot. The inter-trial interval (ITI) was $2000 \mathrm{~ms}$ and $1000 \mathrm{~ms}$ long for successful and aborted trials, respectively. In half of the trials, $500 \mathrm{~ms}$ after eye fixation was acquired one current-pulse train (see section 3.2.3 for stimulation parameters) was delivered and the online, MATLAB-based representation of the eye position and the infrared camera images were carefully monitored to detect eye movements including eye blinks. The current strength started at $50 \mu \mathrm{A}$ and was increased in steps of $50 \mu \mathrm{A}$ after each block without obvious stimulation-induced eye movements until the final current strength of 200 or $250 \mu \mathrm{A}$ was reached. As soon as obvious eye movements time-locked to the delivery of the pulse train were observed the electrode was retrieved by approximately $0.25 \mathrm{~mm}$ and the respective block was repeated. This procedure was repeated until an electrode position was found that allowed stimulation with a current strength of at least $200 \mu \mathrm{A}$ without evoking eye movements.

\section{Distractor task}

Fig. 2.3 shows a schematic of the distractor task. Each trial started with the presentation of a dark grey fixation spot. The animal initiated each trial by acquiring eye fixation within 500 $\mathrm{ms}$ after the onset of the fixation spot. As soon as the animal's eye position entered the $5^{\circ}$ radial window around the fixation spot, the fixation spot turned bright grey. After maintaining fixation for 500-900 ms the fixation spot turned dark again and the animal was presented with one or two peripheral dots representing potential options for an upcoming saccade. This luminance change of the fixation spot with the simultaneous onset of the peripheral visual stimuli represented the time the animal was allowed to give a response (go signal). Red 
dots represented target stimuli whereas yellow and orange dots represented distractors. In conditions with a single peripheral stimulus either one target or one distractor was presented in the left or the right hemifield and the monkey was required to make saccades to targets while ignoring distractors by maintaining central fixation. In conditions with two peripheral stimuli the monkey was shown two dots in opposite hemifields. In double-target trials two equally-rewarded targets were presented and the animal could freely choose either one of them as a saccade target. In double-distractor trials two distractors were presented which had to be ignored by maintaining central fixation. In target-distractor trials a target was presented with a distractor and the animal was required to make a saccade towards the target while ignoring the distractor. Targets and distractors could be presented contralateral to the side of stimulation (left, contraversive side) or ipsilateral to the side of stimulation (right, ipsiversive side). The animal had to make his choice within $500 \mathrm{~ms}$ (target acquisition epoch). As soon as the eye position entered the $5^{\circ}$ radial window around one of the peripheral stimuli the stimulus was considered to be selected and the monkey was not allowed to reverse his decision. Note that in each condition the animal had to choose between making a saccade to one of the peripheral dots and ignoring the peripheral stimuli by maintaining eye fixation resulting in two response options for conditions with one peripheral stimulus (saccade, fixation) and three response options for conditions with two peripheral stimuli (contraversive saccade, ipsiversive saccade, fixation). The selected stimulus, either the selected peripheral dot for saccade responses or the fixation spot for maintaining eye fixation, turned bright to confirm the animal's selection. After fixating the selected stimulus for another $500 \mathrm{~ms}$ (target hold epoch) correct responses were followed by a feedback sound, a fluid reward, and an ITI of $2000 \mathrm{~ms}$ whereas after incorrect responses the next trial started after an ITI of $1000 \mathrm{~ms}$.

All stimuli were matched in luminance (dim stimuli: $11 \mathrm{~cd} / \mathrm{m}^{2}$, bright stimuli: $35 \mathrm{~cd} / \mathrm{m}^{2}$ ) and size $\left(1^{\circ}\right.$ diameter). Targets and distractors were displayed at one of three locations per hemifield (six locations in total) with an eccentricity of $20^{\circ}$ of visual angle. Stimulus locations were arranged concentrically around the fixation spot at $0^{\circ}$ (mid left), $20^{\circ}$ (up left), $160^{\circ}$ (up right), $180^{\circ}$ (mid right), $200^{\circ}$ (down right), and $340^{\circ}$ (down left). In conditions with two peripheral dots the two stimuli were presented either on a horizontal or a diagonal axis. All experimental conditions were pseudorandomly interleaved in such a way that trials that were aborted before the monkey selected a stimulus returned to the pool of trials from which the next trial was chosen randomly. Distractor colors were determined in six initial sessions of psychophysical assessment (see section 2.2.7). In a total number of 16 stimulation sessions in half of the trials electrical microstimulation of the right dPul started either $80 \mathrm{~ms}$ before the go signal (early stimulation condition), simultaneously with the go signal (stimulation at 


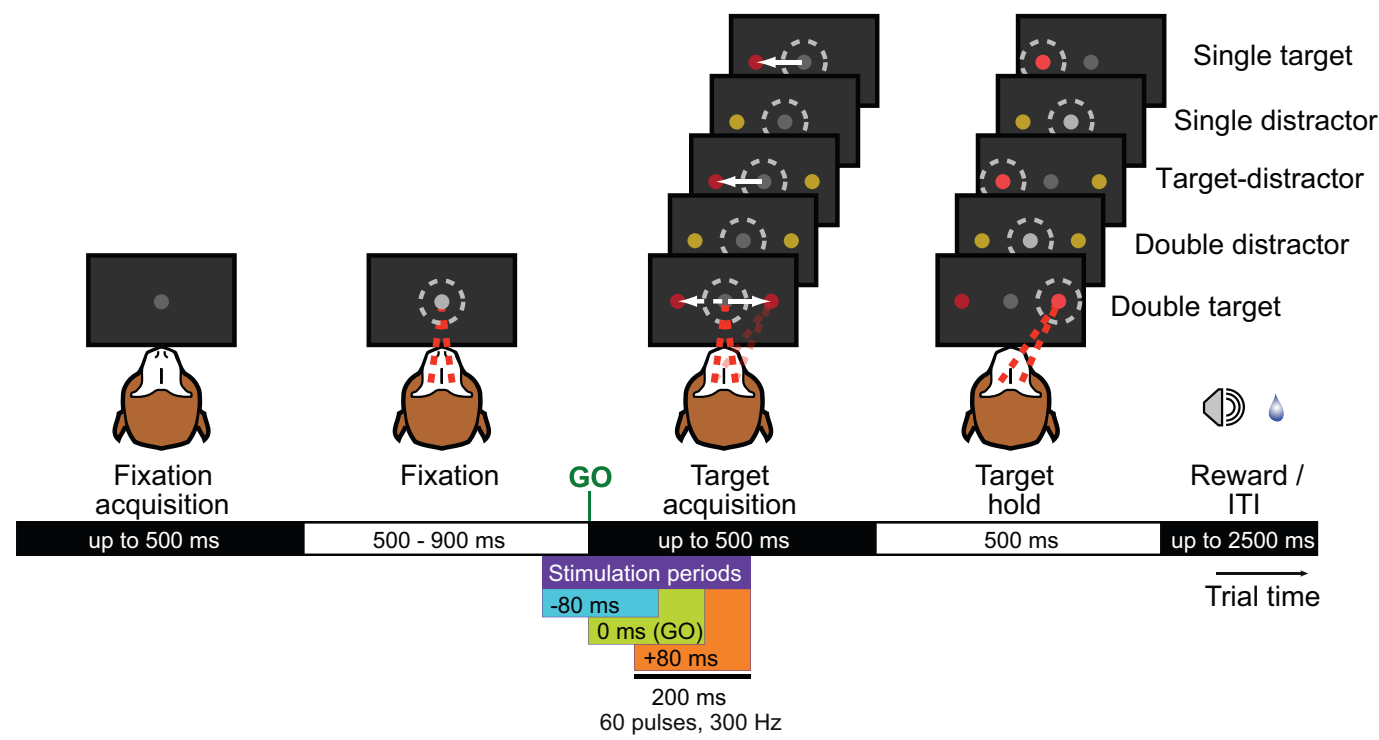

Fig. 2.3 Time course of one trial of the distractor task paradigm. After an initial period of central eye fixation the animal was presented with one or two peripheral stimuli. Stimulus onset together with the dimming of the fixation spot (grey dot) was the go signal for the animal to give the response. Dashed circles represent the animal's eye position in a successful trial for each condition with the radial tolerance window around the stimulus that is currently being looked at. Red dots represent targets, yellow dots represent distractors. Electrical microstimulation was applied either $80 \mathrm{~ms}$ before the go signal $(-80 \mathrm{~ms})$, simultaneously with the go signal $(0 \mathrm{~ms}$, onset of peripheral stimuli), or $80 \mathrm{~ms}$ after the go signal (+80 ms).

go), or $80 \mathrm{~ms}$ after the go signal (late stimulation condition) (see section 3.2.3 for stimulation parameters).

\subsubsection{Distractor color determination}

We determined the two colors used in stimulation sessions based on the results of six initial sessions of psychophysical assessment. In these sessions the animal was performing targetdistractor and double-distractor trials of the distractor task with five distractor colors of different perceptual difficulty ranging from yellow to dark orange. All trial conditions were presented in a randomized order. Perceptual difficulty was defined as the ratio between the proportions of green $(\mathrm{G})$ and red $(\mathrm{R})$ in the color of the peripheral stimuli in RGB color code with the proportion of blue (B) always being fixed at 0 . A stimulus with a $G / R$ ratio of 1 reflects a yellow distractor which is perceptually very different from the target color and thus easy to identify as a distractor whereas a $\mathrm{G} / \mathrm{R}$ ratio of 0 represents the color of the target stimuli. Hence, the lower the $\mathrm{G} / \mathrm{R}$ ratio of a distractor stimulus was the more difficult was the identification of the distractor. Fig. 2.4 shows the psychometric curves for 
target-distractor trials with contraversive targets, ipsiversive targets, and all target positions separately. Psychometric curves were obtained by fitting the hit rate, i.e. the proportion of trials with correct target identification among all trials with a valid response selection, with the cumulative normal function using the Palamedes toolbox (Prins \& Kingdom, 2009) for MATLAB (version R2014b, The MathWorks, Inc., USA). In order to test whether perceptual difficulty had an influence on how pulvinar stimulation affected visual target selection we used the yellow color (distractor $\mathrm{G} / \mathrm{R}$ ratio $=1$ ) as the easy level of perceptual difficulty and a dark orange $(\mathrm{G} / \mathrm{R}$ ratio $=0.18)$ as the difficult level. We used this color for the perceptually difficult condition because with this level of perceptual difficulty the animal still performed above chance level (hit rate above 0.5 ) while ceiling effects were avoided, leaving enough room for behavioral modulation by pulvinar stimulation.

\subsubsection{Data analysis}

All eye movements with a minimum velocity of $200 \% \mathrm{~s}$ and a minimum duration of $30 \mathrm{~ms}$ were considered as saccades. The point in time when eye velocity passed the minimum velocity criterion determined saccade onset. Saccade offset was defined as the point in time

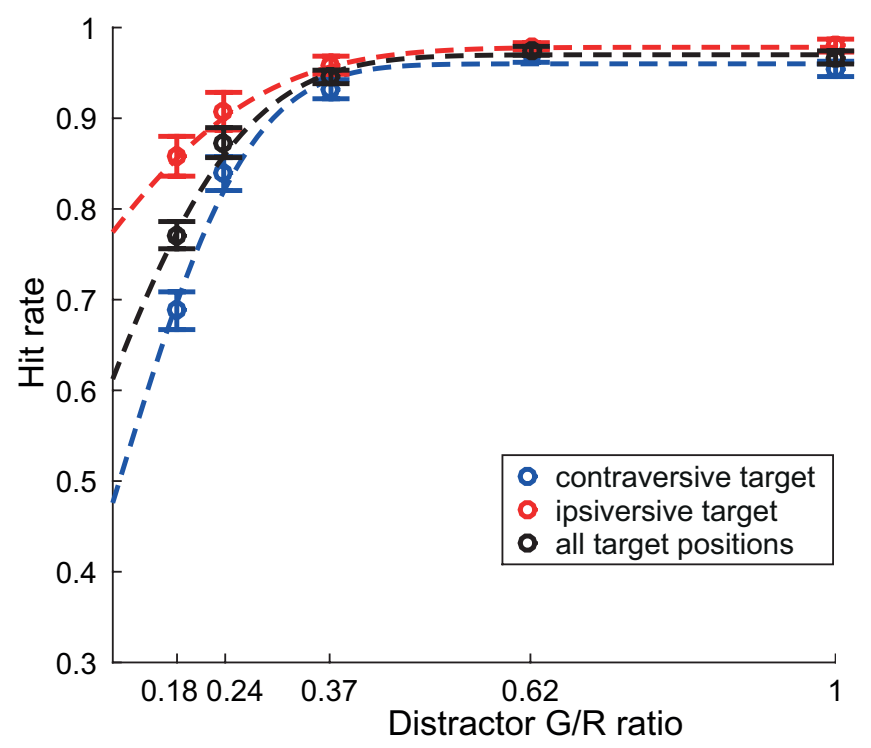

Fig. 2.4 Psychometric curves for distractor color determination in target-distractor trials. Blue curve: trials with a contraversive target, red curve: trials with an ipsiversive target, black curve: all target positions combined, hit rate: proportion of trials with correct target identification among all trials with a valid response selection. The two distractor colors represented by distractor G/R ratios of 0.18 (dark orange, difficult distractor) and 1 (yellow, easy distractor) were later used in stimulation sessions. 
when eye velocity dropped below $50 \%$ s. A trial was completed as soon as the animal either selected one of the peripheral stimuli or the fixation spot (see section 2.2.6). Hit rates were calculated as the proportion of correct trials among all completed trials. Reaction times (RTs) were measured as the time between target onset and saccade onset. Data analysis and statistical tests were performed using MATLAB R2014a (The MathWorks, Inc., USA). Trials were separated by the hemifield in which target or distractor were presented (contraversive or ipsiversive side relative to the side of stimulation) and averaged across all three contraversive and ipsiversive peripheral stimulus positions, respectively. Generally, behavioral effects were first tested using 2-way and 3-way repeated-measures ANOVAs (rmANOVAs, see below). Significant interaction effects were further investigated using paired-sample post-hoc t tests applying the False Discovery Rate (FDR) method to correct for multiple comparisons (Benjamini \& Hochberg, 1995).

\section{Response selection}

To analyze how electrical microstimulation affected response selection (saccade to contraversive stimulus, saccade to ipsiversive stimulus, or central fixation) we calculated the probability of selecting a response among all completed trials for each experimental condition: single contraversive target, single ipsiversive target, single easy contraversive distractor, single difficult contraversive distractor, single easy ipsiversive distractor, single difficult ipsiversive distractor, contraversive target/easy ipsiversive distractor, contraversive target/difficult ipsiversive distractor, ipsiversive target/easy contraversive distractor, ipsiversive target/difficult contraversive distractor, double easy distractor, double difficult distractor, and double target, each condition without stimulation (control), with stimulation before the go signal, with stimulation at the go signal, or with stimulation after the go signal, respectively. Probabilities were calculated for each session separately and means and standard errors of means were calculated across sessions $(n=16)$.

Stimulation effects on response selection were investigated using three different rmANOVA designs. (1) Response selection in double-distractor and double-target trials were analyzed using a two-way rmANOVA with factors stimulus condition (easy double distractor, difficult double distractor, double target) and stimulation (control, before go, at go, after go). (2) Response selection in target-distractor trials were analyzed using a three-way rmANOVA with factors stimulus condition (contraversive target/ipsiversive distractor, ipsiversive target/contraversive distractor), perceptual difficulty (easy, difficult), and stimulation (control, before go, at go, after go). (3) Response selection in single-distractor and single-target trials were analyzed using a three-way rmANOVA with factors stimulus condition (single target, single easy distractor, single difficult distractor), hemifield of stimulus presentation 
(contraversive, ipsiversive), and stimulation (control, before go, at go, after go). For conditions with two peripheral potential saccade options rmANOVAs were applied for each of the three possible response types separately (saccade to contraversive stimulus, saccade to ipsiversive stimulus, or central fixation). The rmANOVA for conditions with only one peripheral potential saccade option was applied on the probability of a saccade response. Note that in these conditions the two possible response types are complementary to each other. Thus, the rmANOVA on the probability of selecting the fixation response reveals the same results. Since our main interest was to investigate the effects of pulvinar stimulation starting at different time points relative to the go signal compared to the control condition without stimulation, significant interaction effects were further analyzed using paired-sample t tests for the three relevant comparisons between stimulation conditions (control vs. stimulation before go, control vs. stimulation at go, and control vs. stimulation after go) for each stimulus condition separately. Resulting $p$ values were adjusted by FDR correction.

\section{Saccade reaction times}

To investigate whether electrical microstimulation delayed or facilitated saccade initiation we analyzed RTs in correct trials with saccades to targets for all experimental conditions except for conditions where no target was presented (single-distractor and double-distractor trials). First, for each session RTs were averaged across all trials of the same experimental condition. Then, for statistical analysis mean RTs and standard errors of means were calculated across sessions. In addition, in order to correct for general differences in the execution of saccades to contraversive and ipsiversive stimuli and to further quantify the magnitude of pulvinar stimulation effects on saccade RTs we subtracted control RTs from stimulation RTs for each stimulation condition per session and calculated mean RT differences and standard errors of mean RT differences across sessions for further statistical analysis.

Stimulation effects on mean RTs and mean RT differences were investigated using two different rmANOVA designs, respectively. (1) RTs in single-target trials were analyzed using a two-way rmANOVA with factors hemifield of stimulus presentation (contraversive, ipsiversive) and stimulation (control, before go, at go, after go). (2) RTs in double-target and target-distractor trials were analyzed using a three-way rmANOVA with factors stimulus condition (double target, target-distractor), hemifield of selected target (contraversive saccade, ipsiversive saccade), and stimulation (control, before go, at go, after go). The latter rmANOVA was applied for both levels of perceptual difficulty separately. Similar to the analysis of response selection, interaction effects were further analyzed using paired-sample $t$ tests with FDR correction for the three comparisons between stimulation conditions. 


\section{Error rates}

We further investigated whether microstimulation affected task performance by calculating the proportion of fixation breaks (trials that were aborted during the inital fixation epoch) and trials with no valid response (trials that were aborted during the target acquisition epoch) among all trials. Error rates were arcsine square root transformed and analyzed using the same rmANOVA designs as were used for analysis of response selection.

\subsection{Results}

\subsubsection{Response selection}

The two-way rmANOVA with factors stimulus condition (easy double distractor, difficult double distractor, double target) and stimulation (control, before go, at go, after go) on the probability of selecting the contraversive peripheral stimulus by making a contraversive saccade in double-distractor and double-target trials revealed significant main effects of stimulus condition and stimulation and a significant stimulus condition $\times$ stimulation interaction effect (see Table 2.1, upper panel, top row). As can be seen in Fig. 2.5A, for difficult double-distractor trials further post-hoc $t$ tests showed a significant decrease in the proportion of contraversive saccades when pulvinar stimulation started before the go signal compared to control trials without stimulation $(t(15)=2.47, p<0.05)$ whereas the proportion of contraversive saccades was significantly increased when stimulation started with or after the go signal $(t(15)=-2.36, p<0.05$ and $t(15)=-2.87, p<0.05$, respectively). Similarly, in double-target trials the animal selected the contraversive target less often than in control trials when stimulation started before the go signal $(t(15)=5.11, p<0.0001)$. In contrast, no significant effects of pulvinar stimulation on contraversive saccade responses were found for easy double-distractor trials (all $t \mathrm{~s}(15) \leq 1.88$, all $p \mathrm{~s} \geq 0.12$ ) except for a decrease in the proportion of contraversive saccades for stimulation after the go signal that did not reach significance after FDR correction $(t(15)=2.22$, uncorrected $p=0.04$, corrected $p=0.12)$. The three-way rmANOVA with factors stimulus condition (contraversive target/ipsiversive distractor, ipsiversive target/ contraversive distractor), perceptual difficulty (easy, difficult), and stimulation (control, before go, at go, after go) on the probability of making a saccade to the contraversive peripheral stimulus in target-distractor trials revealed significant main effects of all three factors. In addition, all possible interaction effects reached significance (see Table 2.1, upper panel, bottom row). Similar to the response selection in difficult doubledistractor trials, post-hoc $\mathrm{t}$ tests showed that there was a lower proportion of correct saccades to contraversive targets presented with a difficult ipsiversive distractor when pulvinar stimu- 
Table 2.1 Results of repeated-measures ANOVAs on the proportions of the three different response types in double-distractor and double-target conditions and in target-distractor conditions.

\begin{tabular}{|c|c|c|c|c|c|}
\hline Response type & Stimulus conditions & Source of variation & $F$ & $d f$ & $p$ \\
\hline \multirow{10}{*}{$\begin{array}{l}\text { Contraversive } \\
\text { saccades }\end{array}$} & \multirow{3}{*}{$\begin{array}{l}\text { Easy double distractor, } \\
\text { difficult double distractor, } \\
\text { double target }\end{array}$} & Stimulus condition & 123.26 & 2,30 & $3.44 \times 10^{-15}$ \\
\hline & & Stimulation & 10.00 & 3,45 & $3.63 \times 10^{-5}$ \\
\hline & & Stimulus condition $\times$ stimulation & 7.39 & 6,90 & $1.90 \times 10^{-6}$ \\
\hline & \multirow{7}{*}{$\begin{array}{l}\text { Contraversive target/, } \\
\text { ipsiversive distractor, } \\
\text { ipsiversive target/ } \\
\text { contraversive distractor }\end{array}$} & Stimulus condition & $3.10 \times 10^{3}$ & 1,15 & 0.0000 \\
\hline & & Difficulty & 82.49 & 1,15 & $1.75 \times 10^{-7}$ \\
\hline & & Stimulation & 25.44 & 3,45 & $8.88 \times 10^{-10}$ \\
\hline & & Stimulus condition $\times$ difficulty & 822.93 & 1,15 & $1.61 \times 10^{-14}$ \\
\hline & & Stimulus condition $\times$ stimulation & 22.67 & 3,45 & $4.30 \times 10^{-9}$ \\
\hline & & Difficulty $\times$ stimulation & 16.61 & 3,45 & $2.10 \times 10^{-7}$ \\
\hline & & Stimulus condition $\times$ difficulty $\times$ stimulation & 8.63 & 3,45 & $1.23 \times 10^{-4}$ \\
\hline \multirow{10}{*}{$\begin{array}{l}\text { Ipsiversive } \\
\text { saccades }\end{array}$} & \multirow{3}{*}{$\begin{array}{l}\text { Easy double distractor, } \\
\text { difficult double distractor, } \\
\text { double target }\end{array}$} & Stimulus condition & $1.07 \times 10^{3}$ & 2,30 & 0.0000 \\
\hline & & Stimulation & 34.48 & 3,45 & $9.98 \times 10^{-12}$ \\
\hline & & Stimulus condition $\times$ stimulation & 4.14 & 6,90 & 0.0010 \\
\hline & \multirow{7}{*}{$\begin{array}{l}\text { Contraversive target/, } \\
\text { ipsiversive distractor, } \\
\text { ipsiversive target/ } \\
\text { contraversive distractor }\end{array}$} & Stimulus condition & $5.49 \times 10^{3}$ & 1,15 & 0.0000 \\
\hline & & Difficulty & 134.55 & 1,15 & $6.86 \times 10^{-9}$ \\
\hline & & Stimulation & 35.65 & 3,45 & $5.93 \times 10^{-12}$ \\
\hline & & Stimulus condition $\times$ difficulty & 692.36 & 1,15 & $5.74 \times 10^{-14}$ \\
\hline & & Stimulus condition $\times$ stimulation & 19.60 & 3,45 & $2.83 \times 10^{-8}$ \\
\hline & & Difficulty $\times$ stimulation & 14.40 & 3,45 & $1.03 \times 10^{-6}$ \\
\hline & & Stimulus condition $\times$ difficulty $\times$ stimulation & 8.71 & 3,45 & $1.15 \times 10^{-4}$ \\
\hline \multirow{10}{*}{ Fixation } & \multirow{3}{*}{$\begin{array}{l}\text { Easy double distractor, } \\
\text { difficult double distractor, } \\
\text { double target }\end{array}$} & Stimulus condition & $1.90 \times 10^{3}$ & 2,30 & 0.0000 \\
\hline & & Stimulation & 37.07 & 3,45 & $3.20 \times 10^{-12}$ \\
\hline & & Stimulus condition $\times$ stimulation & 17.32 & 6,90 & $3.21 \times 10^{-13}$ \\
\hline & \multirow{7}{*}{$\begin{array}{l}\text { Contraversive target/, } \\
\text { ipsiversive distractor, } \\
\text { ipsiversive target/ } \\
\text { contraversive distractor }\end{array}$} & Stimulus condition & 5.82 & 1,15 & 0.0291 \\
\hline & & Difficulty & 19.64 & 1,15 & $4.85 \times 10^{-4}$ \\
\hline & & Stimulation & 6.06 & 3,45 & 0.0015 \\
\hline & & Stimulus condition $\times$ difficulty & 5.94 & 1,15 & 0.0277 \\
\hline & & Stimulus condition $\times$ stimulation & 3.54 & 3,45 & 0.0220 \\
\hline & & Difficulty $\times$ stimulation & 1.98 & 3,45 & 0.1305 \\
\hline & & Stimulus condition $\times$ difficulty $\times$ stimulation & 2.06 & 3,45 & 0.1194 \\
\hline
\end{tabular}


lation started before the go signal $(t(15)=3.85, p<0.01)$ whereas the proportion of correct contraversive saccades was increased when stimulation started with or after the go signal $(t(15)=-2.70, p<0.05$ and $t(15)=-3.62, p<0.01$, respectively) (see Fig. 2.5C). Even when contraversive targets were presented with an easy ipsiversive distractor stimulation before the go signal led to a significantly decreased proportion of correct contraversive saccades $(t(15)=5.43, p<0.0001)$. Moreover, in this condition stimulation at the go signal also led to a slight decrease in the proportion of correct contraversive saccades $(t(15)=2.45, p<$ 0.05 ) resembling the effect of stimulation before the go signal (see Fig. 2.5B). Also note that in this condition the animal's performance, i.e. the proportion of correct contraversive saccades, was almost perfect in the control condition without pulvinar stimulation leaving very little room for further improvement in performance due to stimulation. There were no significant effects on the proportion of contraversive saccades in trials with ipsiversive targets and contraversive distractors (all $t \mathrm{~s}(15) \leq 2.02$, all $p \mathrm{~s} \geq 0.09$ ) except for a slight increase in contraversive saccades for trials with a difficult distractor and stimulation after the go signal and for trials with an easy distractor and stimulation at the go signal, both, however, not reaching significance after FDR correction $(t(15)=-2.42$, uncorrected $p=0.03$, corrected $p=$ 0.09 and $t(15)=-2.51$, uncorrected $p=0.02$, corrected $p=0.07$, respectively) (see Fig. 2.5B and $\mathrm{C}$ ).

Overall, analysis on the proportion of ipsiversive saccades revealed a pattern of response selection that was complementary to the results found for contraversive saccades. The two-way rmANOVA on the proportion of ipsiversive saccades in double-distractor and double-target trials also showed significant main effects of stimulus condition and stimulation and a significant two-way interaction effect (see Table 2.1, middle panel, top row). As shown in Fig. 2.6A, for all three stimulus conditions, post-hoc $t$ tests showed a significant increase in ipsiversive saccades for pulvinar stimulation starting before the go signal (all $t \mathrm{~s}(15) \geq$ 3.63, all $p s<0.01$ ). Moreover, in both easy and difficult double-distractor trials stimulation at and after the go signal led to a significant decrease in the proportion of ipsiversive saccades (all $t \mathrm{~s}(15) \geq 2.78$, all $p \mathrm{~s}<0.05$ ). Likewise, the three-way rmANOVA on the proportion of ipsiversive saccades in target-distractor trials confirmed significant main effects of stimulus condition, perceptual difficulty, and stimulation. In addition, all possible interaction effects were significant (see Table 2.1, middle panel, bottom row). Fig. 2.6B and Fig. 2.6C show the effects of pulvinar stimulation on ipsiversive saccades in target-distractor trials. Post-hoc $t$ tests revealed a significant increase in the proportion of incorrect ipsiversive saccades in trials with a contraversive target and an easy or a difficult ipsiversive distractor and stimulation starting before the go signal $(t(15)=-6.29, p<0.0001$ and $t(15)=3.69, p<0.01$, respectively) whereas stimulation at and after the go signal led to a significant decrease in the proportion of 

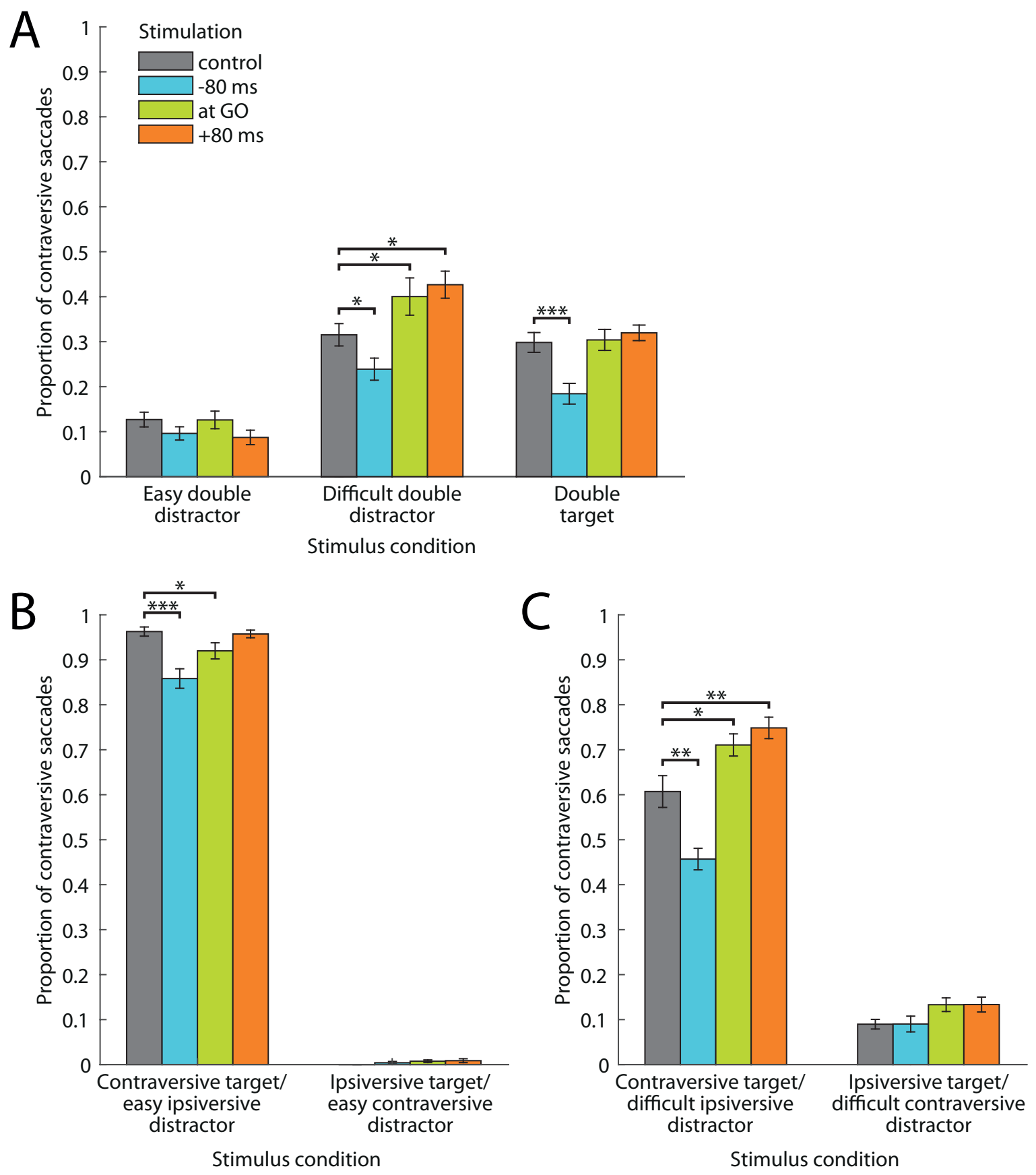

Fig. 2.5 Proportions of contraversive saccades among all trials with valid response selection in (A) double-distractor and double-target conditions, (B) target-distractor conditions with low perceptual difficulty, and (C) target-distractor conditions with high perceptual difficulty. Grey bars: no pulvinar stimulation (control), blue bars: pulvinar stimulation before the go signal $(-80 \mathrm{~ms})$, green bars: pulvinar stimulation at the go signal (at $\mathrm{GO})$, orange bars: pulvinar stimulation after the go signal $(+80 \mathrm{~ms})$. ${ }^{*} \mathrm{p}<0.05, * * \mathrm{p}<0.01, * * * \mathrm{p}<0.001$, FDR corrected. 

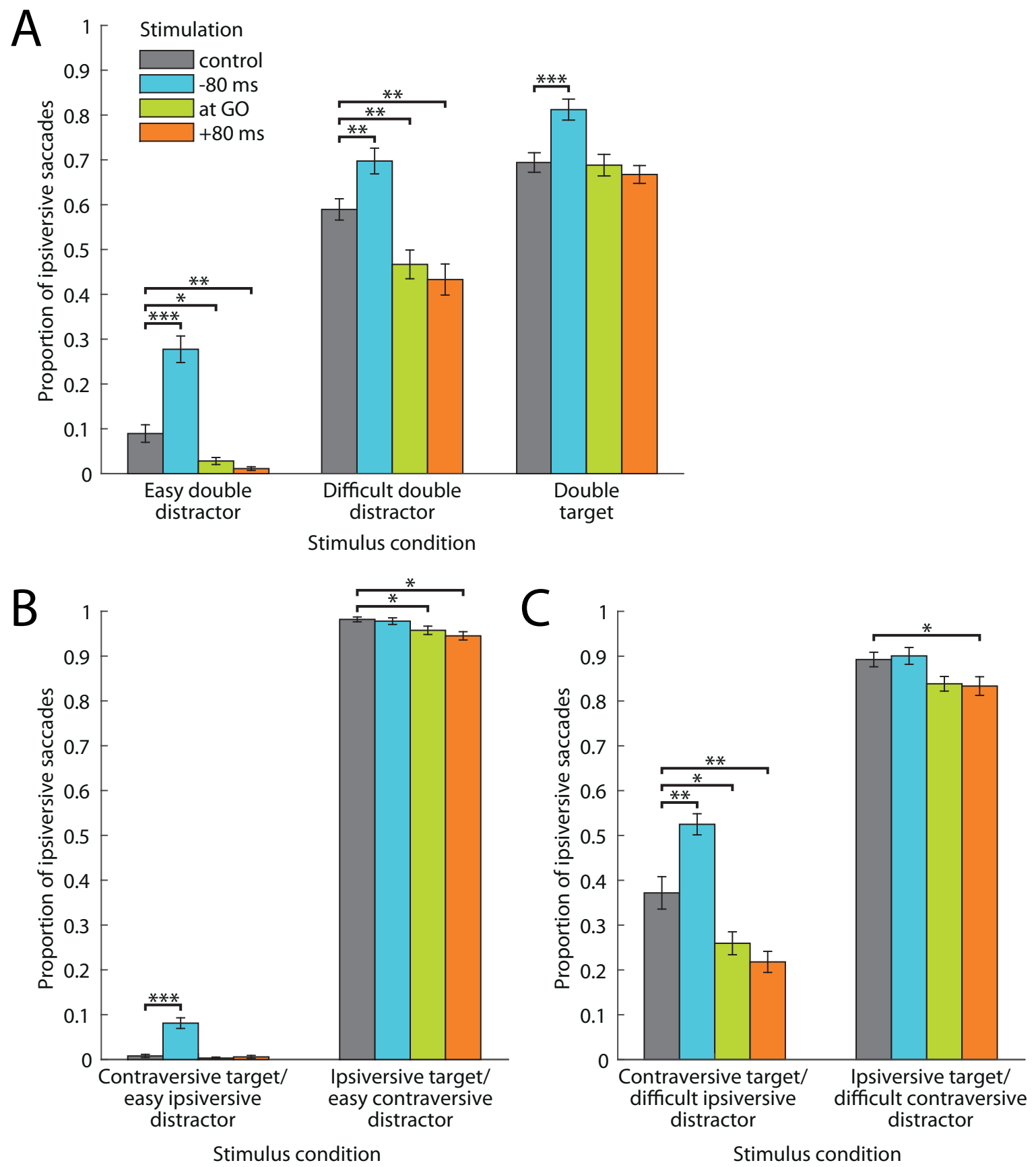

Fig. 2.6 Proportions of ipsiversive saccades among all trials with valid response selection in (A) double-distractor and double-target conditions, (B) target-distractor conditions with low perceptual difficulty, and (C) target-distractor conditions with high perceptual difficulty. Grey bars: no pulvinar stimulation (control), blue bars: pulvinar stimulation before the go signal $(-80 \mathrm{~ms})$, green bars: pulvinar stimulation at the go signal (at $\mathrm{GO})$, orange bars: pulvinar stimulation after the go signal (+80 ms). * $\mathrm{p}<0.05, * * \mathrm{p}<0.01, * * * \mathrm{p}<0.001$, FDR corrected. 
incorrect ipsiversive saccades, i.e. better performance, in trials with contraversive targets and difficult ipsiversive distractors $(t(15)=2.89, p<0.05$ and $t(15)=3.55, p<0.01$, respectively). Similarly, pulvinar stimulation at the go signal was associated with a decreased proportion of ipsiversive saccades in trials with ipsiversive targets and easy contraversive distractors $(t(15)$ $=2.87, p<0.05$ ) and stimulation after the go signal resulted in a decreased proportion of ipsiversive saccades in trials with ipsiversive targets and both easy and difficult contraversive distractors $(t(15)=3.17, p<0.05$ and $t(15)=2.74, p<0.05$, respectively). Note that in the stimulus conditions with ipsiversive targets a decrease in the proportion of ipsiversive saccades reflects a deterioration of performance.

The effects of pulvinar microstimulation on the proportion of fixation responses were rather small with the exception of the easy double-distractor stimulus condition. The twoway rmANOVA on the proportion of fixation responses in double-distractor and doubletarget trials again showed significant main effects of the two factors stimulus condition and stimulation and a significant stimulus condition $\times$ stimulation interaction effect (see Table 2.1, bottom panel, top row). As shown in Fig. 2.7A, this interaction effect was mostly driven by significant stimulation effects in easy double-distractor trials. In this stimulus condition, pulvinar stimulation before the go signal led to a significant decrease in fixation responses, i.e. worse performance $(t(15)=3.92, p<0.01)$ while there was a significant increase in the proportion of fixation responses, i.e. better performance, when stimulation started at or after the go signal $(t(15)=-2.60, p<0.05$ and $t(15)=-5.65, p<0.001$, respectively). A similar improvement in performance due to stimulation at and after the go signal was observed in difficult double-distractor trials which, however, did not reach significance after FDR correction $(t(15)=-2.37$, uncorrected $p=0.03$, corrected $p=0.06$ and $t(15)=-2.29$, uncorrected $p=0.04$, corrected $p=0.06$, respectively). The three-way rmANOVA on the proportion of fixation responses in target-distractor trials revealed significant main effects of all three factors, stimulus condition, perceptual difficulty, and stimulation. Moreover, there was a significant stimulus condition $\times$ perceptual difficulty interaction effect and a significant stimulus condition $\times$ stimulation interaction effect (see Table 2.1, bottom panel, bottom row). These effects were driven by significant effects of pulvinar stimulation on fixation responses in easy target-distractor trials (see Fig. 2.7B) where stimulation at the go signal increased the proportion of fixation responses in trials with a contraversive target and an ipsiversive distractor $(t(15)=-2.77, p<0.05)$ and stimulation after the go increased the proportion of fixation responses in trials with an ipsiversive target and a contraversive distractor $(t(15)=$ $-2.82, p<0.05)$. Note that in both cases an increased proportion of fixation responses reflects a deterioration of performance. There were no significant effects of pulvinar stimulation 

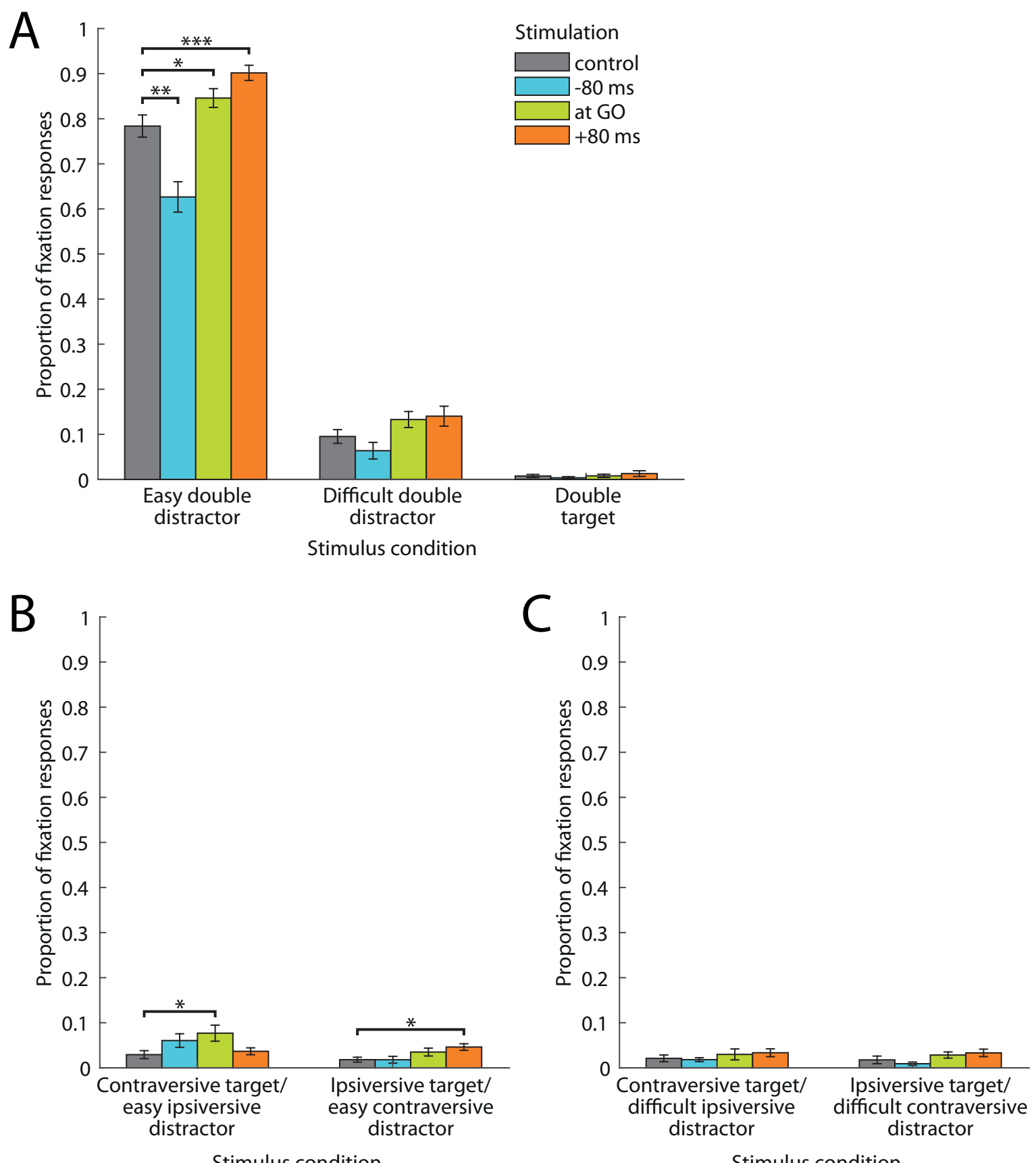

Fig. 2.7 Proportions of fixation responses among all trials with valid response selection in (A) double-distractor and double-target conditions, (B) target-distractor conditions with low perceptual difficulty, and (C) target-distractor conditions with high perceptual difficulty. Grey bars: no pulvinar stimulation (control), blue bars: pulvinar stimulation before the go signal $(-80 \mathrm{~ms})$, green bars: pulvinar stimulation at the go signal (at GO), orange bars: pulvinar stimulation after the go signal $(+80 \mathrm{~ms}) . * \mathrm{p}<0.05$, ** $\mathrm{p}<0.01$, *** $\mathrm{p}<0.001$, FDR corrected. 
Table 2.2 Results of repeated-measures ANOVAs on the proportions of saccade response in single-distractor and single-target conditions.

\begin{tabular}{llccc}
\hline Stimulus conditions & Source of variation & $F$ & $d f$ & $p$ \\
\hline & Stimulus condition & $1.88 \times 10^{3}$ & 2,30 & 0.0000 \\
Single easy distractor, & Semifield & 32.50 & 1,15 & $4.20 \times 10^{-5}$ \\
single difficult distractor, & Stimulation & 63.80 & 3,45 & $3.33 \times 10^{-16}$ \\
single target & Stimulus condition $\times$ hemifield & 25.15 & 2,30 & $3.85 \times 10^{-7}$ \\
& Hemifield $\times$ stimulation & 15.99 & 6,90 & $1.97 \times 10^{-12}$ \\
& Stimulus condition $\times$ hemifield $\times$ stimulation & 2.31 & 6,90 & 0.0407 \\
\hline
\end{tabular}

on fixation responses in difficult target-distractor trials (all $t \mathrm{~s}(15) \leq 1.59$, all $p \mathrm{~s} \geq 0.37$, see Fig. 2.7C)

For conditions with only one peripheral saccade option the three-way rmANOVA with factors stimulus condition (single target, single easy distractor, single difficult distractor), hemifield of stimulus presentation (contraversive, ipsiversive), and stimulation (control, before go, at go, after go) on the probability of making a saccade response towards the peripheral stimulus instead of central fixation revealed significant main effects of all three factors. In addition, all possible interaction effects reached significance (see Table 2.2). Fig. 2.8 shows the proportions of saccade responses for all single-stimulus conditions. Posthoc $t$ tests showed that in these conditions pulvinar stimulation mainly affected the proportion of saccades towards ipsiversive stimuli. More specifically, when an easy or a difficult distractor was presented on the ipsiversive side of space stimulation before the go signal led to a significant increase in incorrect saccade responses towards the ipsiversive distractor $(t(15)=-5.23, p<0.001$ and $t(15)=-4.22, p<0.01$, respectively) whereas stimulation at or after the go signal significantly decreased the proportion of saccades towards both easy and difficult ipsiversive distractors $(t(15)=2.19, p<0.05$ and $t(15)=3.23, p<0.01$ for easy ipsiversive distractors, $t(15)=2.99, p<0.01$ and $t(15)=4.53, p<0.01$ for difficult ipsiversive distractors, respectively). The slight decrease in saccade responses towards difficult contraversive distractors for stimulation after the go signal did not reach significance after FDR correction $(t(15)=2.69$, uncorrected $p=0.02$, corrected $p=0.05)$. When only one target was presented on the ipsiversive side of space pulvinar stimulation after the go signal significantly decreased the proportion of correct saccades towards the ipsiversive target $(t(15)=4.74, p<0.001)$. Note that in this condition the animal's hit rate, i.e. proportion of saccade responses to single ipsiversive targets was already above 0.9 in the control stimulation condition leaving no room for a further increase in the number of ipsiversive saccades. 

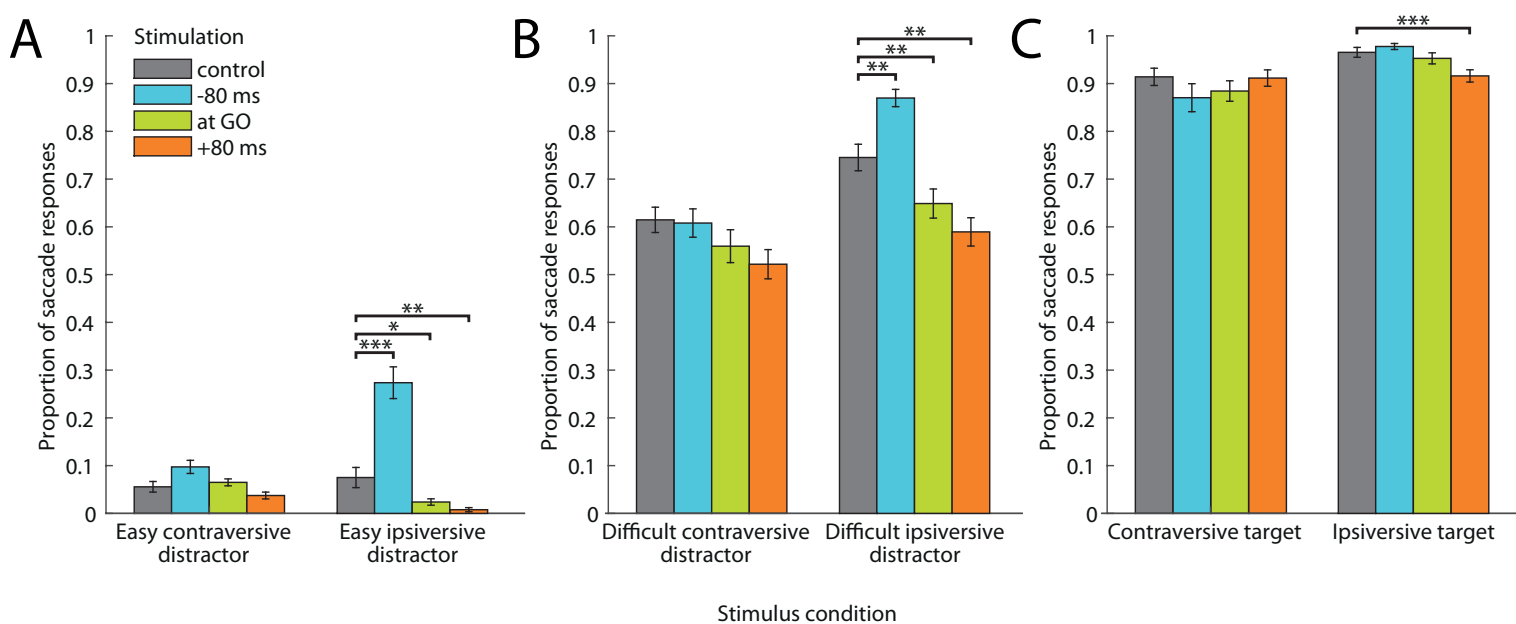

Fig. 2.8 Proportions of saccade responses towards a single peripheral stimulus among all trials with valid response selection in (A) easy single-distractor conditions, (B) difficult singledistractor, and (C) single-target conditions. Grey bars: no pulvinar stimulation (control), blue bars: pulvinar stimulation before the go signal $(-80 \mathrm{~ms})$, green bars: pulvinar stimulation at the go signal (at GO), orange bars: pulvinar stimulation after the go signal (+80 ms). ${ }^{*} \mathrm{p}<$ $0.05, * * \mathrm{p}<0.01, * * * \mathrm{p}<0.001$, FDR corrected.

Taken as a whole, the effect of pulvinar microstimulation strongly depended on the time when the stimulation was delivered relative to the go signal. In addition, these effects were partially independent of the perceptual difficulty of the discrimination between targets and distractors. More specifically, pulvinar stimulation before the go signal led to a consistent decrease in the proportion of saccades towards both contraversive targets and distractors which was accompanied by an increase in the proportion of ipsiversive saccades. In contrast, the most prominent effect of pulvinar stimulation at or after the go signal was an increase in the proportion of contraversive saccades accompanied by a decrease in the proportion of ipsiversive saccades. The effects on ipsiversive saccade responses were similar in conditions with easy and difficult distractors and even extended to single-stimulus conditions where only one saccade option was available on the ipsiversive side. Effects of pulvinar stimulation on the proportion of fixation responses were only observed for conditions with low perceptual difficulty with strongest effects in the condition where two easy distractors were presented. In this condition, stimulation before the go signal led to a decrease in fixation responses whereas stimulation at or after the go was associated with an increase in fixation responses. Note that whether a change in the proportion of one response type reflects an improvement or deterioration of performance depends on the stimulus condition. For instance, the increase in the proportion of ipsiversive saccades due to stimulation before the go signal is related to an improvement in performance only when a target was presented on the ipsiversive side 
of space. When an ipsiversive distractor was presented the increase in the proportion of ipsiversive saccades reflects a deterioration of performance. The same applies to changes in the proportion of contraversive saccades and fixation responses. In the latter case, only in single-distractor and double-distractor conditions an increased proportion of fixation responses reflects an improvement in performance. Thus, pulvinar stimulation was associated with both an improvement and a deterioration of performance.

\subsubsection{Saccade reaction times}

The two-way rmANOVA with factors hemifield of stimulus presentation (contraversive, ipsiversive) and stimulation (control, before go, at go, after go) on mean saccade RTs in single-target conditions revealed main effects of both factors and a significant hemifield $x$ stimulation interaction effect (see Table 2.3, upper panel). As shown in Fig. 2.9A, pulvinar stimulation before the go signal led to significantly shorter RTs of correct saccades to both contraversive and ipsiversive targets than in control trials $(t(15)=13.20, p<0.0001$ and $t(15)$ $=8.77, p<0.0001$, respectively) whereas stimulation after the go signal significantly delayed correct saccades to both contraversive and ipsiversive targets $(t(15)=-3.16, p<0.01$ and $t(15)$ $=-4.71, p<0.001$, respectively). In addition, reaction times of correct saccades to ipsiversive targets were significantly longer when stimulation started at the go signal compared to control trials $(t(15)=-2.91, p<0.05)$. The two-way rmANOVA on mean RT differences between stimulation before, at, or after the go signal and the control condition confirmed the main effects of hemifield of stimulus presentation and stimulation (see Table 2.4, upper panel) but the hemifield $\times$ stimulation interaction did not reach significance indicating that the magnitudes of saccade facilitation and delay were quite similar between saccades to single contraversive and ipsiversive targets.

For double-target and easy target-distractor trials the three-way rmANOVA with factors stimulus condition (double target, target-distractor), hemifield of selected target (contraversive saccade, ipsiversive saccade), and stimulation (control, before go, at go, after go) on mean RTs revealed significant main effects of stimulation and hemifield of selected target and a significant hemifield $\times$ stimulation interaction effect (see Table 2.3, middle panel). Fig. 2.9B and Fig. 2.9C show mean saccade RTs for double-target and easy target-distractor trials, respectively. In both stimulus conditions, pulvinar stimulation before the go signal led to significantly shorter ipsiversive saccade RTs (target-target condition: $t(15)=5.84, p<$ 0.0001 , target-distractor condition: $t(15)=9.31, p<0.0001$ ) whereas stimulation at or after the go signal resulted in significantly longer ipsiversive saccade RTs compared to control trials (all $t \mathrm{~s}(15) \leq-3.23$, all $p \mathrm{~s}<0.01$ ). Correct saccades to contraversive targets were also significantly delayed by stimulation after the go signal in both stimulus conditions 
Table 2.3 Results of repeated-measures ANOVAs on mean saccade reaction times.

\begin{tabular}{llccc}
\hline Stimulus conditions & Source of variation & $F$ & $d f$ & $p$ \\
\hline \multirow{3}{*}{ Single target } & Hemifield & 189.81 & 1,15 & $6.41 \times 10^{-10}$ \\
& Stimulation & 50.95 & 3,45 & $1.63 \times 10^{-14}$ \\
& Hemifield $\times$ stimulation & 4.77 & 3,45 & 0.0057 \\
\hline & Stimulus condition & 3.89 & 1,15 & 0.0672 \\
& Hemifield & 143.95 & 1,15 & 0.0000 \\
Double target, & Stimulation & 33.24 & 3,45 & 0.0000 \\
target/easy distractor & Stimulus condition $\times$ hemifield & 1.41 & 1,15 & 0.2543 \\
& Stimulus condition $\times$ stimulation & 1.77 & 3,45 & 0.1673 \\
& Hemifield $\times$ stimulation & 8.18 & 3,45 & $1.87 \times 10^{-4}$ \\
& Stimulus condition $\times$ hemifield $\times$ stimulation & 2.14 & 3,45 & 0.1081 \\
\hline \multirow{5}{*}{ Double target, } & Stimulus condition & 0.29 & 1,15 & 0.6003 \\
target/difficult distractor & Stimulus condition $\times$ hemifield & 112.80 & 1,15 & 0.0000 \\
& Stimulus condition $\times$ stimulation & 31.61 & 3,45 & 0.0000 \\
& Hemifield & 4.21 & 1,15 & 0.0580 \\
& Stimulation stimulation & 0.56 & 3,45 & 0.6460 \\
& Stimulus condition $\times$ hemifield $\times$ stimulation & 6.47 & 3,45 & $9.77 \times 10^{-4}$ \\
& & 0.66 & 3,45 & 0.5790 \\
\hline
\end{tabular}

Table 2.4 Results of repeated-measures ANOVAs on mean saccade reaction time differences between the control condition without pulvinar microstimulation and microstimulation before, at, or after the go signal.

\begin{tabular}{llccc}
\hline Stimulus conditions & Source of variation & $F$ & $d f$ & $p$ \\
\hline \multirow{3}{*}{ Single target } & Hemifield & 19.68 & 1,15 & $4.81 \times 10^{-4}$ \\
& Stimulation & 71.98 & 2,30 & $3.55 \times 10^{-12}$ \\
& Hemifield $\times$ stimulation & 0.26 & 2,30 & 0.7755 \\
\hline & Stimulus condition & 3.98 & 1,15 & 0.0645 \\
& Hemifield & 0.05 & 1,15 & 0.8330 \\
Double target, & Stimulation & 47.05 & 2,30 & 0.0000 \\
target/easy distractor & Stimulus condition $\times$ hemifield & 7.42 & 1,15 & 0.0157 \\
& Stimulus condition $\times$ stimulation & 1.15 & 2,30 & 0.3309 \\
& Hemifield $\times$ stimulation & 11.77 & 2,30 & $1.68 \times 10^{-4}$ \\
& Stimulus condition $\times$ hemifield $\times$ stimulation & 0.26 & 2,30 & 0.7699 \\
\hline & Stimulus condition & 1.29 & 1,15 & 0.2740 \\
Double target, & Hemifield & 1.66 & 1,15 & 0.2174 \\
target/difficult distractor & Stimulation & 45.31 & 2,30 & 0.0000 \\
& Stimulus condition $\times$ hemifield & 0.70 & 1,15 & 0.4148 \\
& Stimulus condition $\times$ stimulation & 0.31 & 2,30 & 0.7336 \\
& Hemifield $\times$ stimulation & 9.18 & 2,30 & $7.76 \times 10^{-4}$ \\
& Stimulus condition $\times$ hemifield $\times$ stimulation & 0.62 & 2,30 & 0.5463 \\
\hline
\end{tabular}


(target-target condition: $t(15)=-4.63, p<0.001$, target-distractor condition: $t(15)=-4.31$, $p<0.0001)$. Additionally, stimulation at the go signal led to a significant delay of correct saccades to contraversive targets in double-target trials $(t(15)=-3.23, p<0.01)$ and, similar to ipsiversive saccade RTs, stimulation before the go signal significantly reduced RTs of saccades to contraversive targets in easy target-distractor trials $(t(15)=7.88, p<0.0001)$. The three-way rmANOVA on mean RT differences confirmed the significant main effect of stimulation and the significant hemifield $\times$ stimulation interaction effect. In addition, the interaction effect between stimulus condition and hemifield reached significance whereas the main effect of hemifield was not significant (see Table 2.4, middle panel). To further investigate the hemifield $\times$ stimulation interaction effect, we applied post-hoc $t$ tests on the mean RT differences averaged across stimulus conditions which showed a significantly longer delay of ipsiversive saccades compared to contraversive saccades when pulvinar stimulation started at the go signal ( $M=18 \mathrm{~ms}, S E=5 \mathrm{~ms}$ and $M=9 \mathrm{~ms}, S E=3 \mathrm{~ms}$, respectively, $t(15)$ $=-3.90, p<0.01)$.

Results of the three-way rmANOVA on mean RTs in double-target and difficult targetdistractor trials were very similar to the results obtained for double-target and easy targetdistractor trials with significant main effects of stimulation and hemifield of selected target and a significant hemifield $\times$ stimulation interaction effect (see Table 2.3, bottom panel). As can be seen in Fig. 2.9D, similar to all other stimulus conditions, RTs of saccades to ipsiversive targets in difficult target-distractor trials were significantly shorter for stimulation before the go signal than in control trials $(t(15)=11.46, p<0.0001)$ whereas stimulation at and after the go signal led to significant delays of ipsiversvie saccade RTs $(t(15)=-3.97, p<$ 0.01 and $t(15)=-4.40, p<0.001$, respectively). Stimulation at and after the go signal also significantly delayed saccades to contraversive targets $(t(15)=-3.06, p<0.05$ and $t(15)=$ $-4.43, p<0.01$, respectively), resembling the effects of pulvinar stimulation in double-target conditions. The three-way rmANOVA on mean RT differences resembled the effects found for mean RTs for the most part and were also similar to the results obtained for double-target and easy target-distractor trials. More specifically, there was a significant main effect of stimulation and a significant hemifield $\times$ stimulation interaction effect. In contrast, the main effect of hemifield was not significant (see Table 2.4, bottom panel). However, for double-target and difficult target-distractor trials post-hoc t tests applied on the mean RT differences averaged across stimulus conditions showed a significantly stronger facilitation of ipsiversive saccades compared to contraversive saccades for stimulation before the go signal $(M=-19 \mathrm{~ms}, S E=2 \mathrm{~ms}$ and $M=-6 \mathrm{~ms}, S E=5 \mathrm{~ms}$, respectively, $t(15)=2.76, p<$ $0.05)$. 

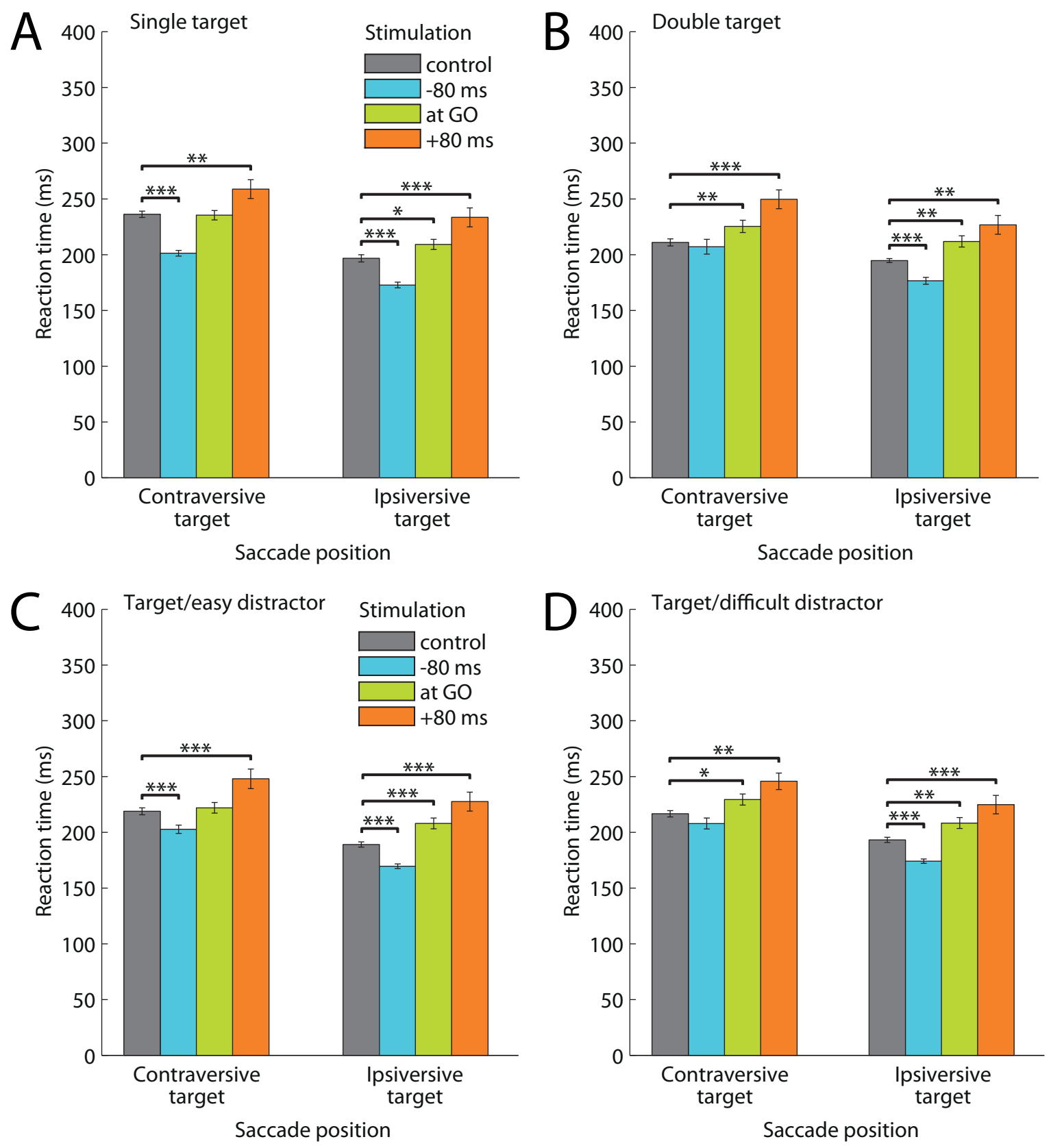

Fig. 2.9 Saccade reaction times for correct saccades to contraversive and ipsiversive targets in (A) single-target conditions, (B) double-target conditions, (C) conditions with a target and an easy distractor, and (D) conditions with a target and a difficult distractor. Grey bars: no pulvinar stimulation (control), blue bars: pulvinar stimulation before the go signal (-80 ms), green bars: pulvinar stimulation at the go signal (at GO), orange bars: pulvinar stimulation after the go signal $(+80 \mathrm{~ms}) .{ }^{*} \mathrm{p}<0.05, * * \mathrm{p}<0.01, * * * \mathrm{p}<0.001$, FDR corrected. 
Overall, similar to pulvinar stimulation effects on response selection, effects of pulvinar stimulation on saccade RTs were dependent on the time when stimulation was applied relative to the go signal. Moreover, RT effects were quite consistent across stimulus conditions, especially for saccades to ipsiversive targets. Here, stimulation before the go signal consistently reduced saccade RTs whereas stimulation at and after the go signal consistently delayed ipsiversive saccades. Also consistent across stimulus conditions and similar to the effects on ipsiversive saccade RTs, stimulation after the go signal also increased RTs of saccades to contraversive targets. In single-target and easy target-distractor trials the facilitatory effect of stimulation before the go signal was also found for contraversive saccades and stimulation at the go signal significantly delayed saccades to contraversive targets in double-target and difficult target-distractor trials. The magnitudes of saccade facilitation and delay of ipsiversive and contraversive saccades were quite similar except for a longer delay of ipsiversive saccades compared to contraversive saccades when pulvinar stimulation started at the go signal averaged across double-target and easy target-distractor trials. Moreover, a stronger facilitation of ipsiversive saccades compared to contraversive saccades was found for pulvinar stimulation before the go signal when averaging across double-target and difficult target-distractor trials.

\subsubsection{Error rates}

None of the rmANOVAs on the proportion of fixation breaks revealed significant effects of pulvinar stimulation (see Table 2.5 and Table 2.6, upper panel, respectively). However, the three-way rmANOVA on the proportion of trials with no valid response selection in targetdistractor conditions showed a significant interaction effect between the three factors stimulus condition, perceptual difficulty, and stimulation. Post-hoc $\mathrm{t}$ tests revealed significantly higher proportions of trials with no valid response selection for easy target-distractor trials with an ipsiversive target and pulvinar stimulation at the go signal or after the go signal compared to control trials without stimulation (Control: $M=0, S E=0$, Stimulation at go signal: $M=$ $0.06, S E=0.02$, Stimulation after go signal: $M=0.05, S E=0.02, t(15)=-2.96$ and $t(15)$ $=-2.59$, both $p \mathrm{~s}<0.05$ ). In addition, the three-way rmANOVA on the proportion of trials with no valid response selection in single-distractor and single-target conditions revealed a significant main effect of stimulation (see Table 2.6, bottom panel) which was driven by a higher proportion of no valid response selection in trials with a single easy distractor presented on the ipsiversive side and pulvinar stimulation after the go signal compared to control trials (Control: $M=0.05, S E=0.02$, Stimulation after go signal: $M=0.12, S E=$ $0.02, t(15)=-3.07, p<0.05)$. 
Table 2.5 Results of repeated-measures ANOVAs on the proportions of two different error types among all trials in double-distractor and double-target conditions and in target-distractor conditions.

\begin{tabular}{|c|c|c|c|c|c|}
\hline Error type & Stimulus conditions & Source of variation & $F$ & $d f$ & $p$ \\
\hline \multirow{10}{*}{$\begin{array}{l}\text { Fixation } \\
\text { breaks }\end{array}$} & \multirow{3}{*}{$\begin{array}{l}\text { Easy double distractor, } \\
\text { difficult double distractor, } \\
\text { double target }\end{array}$} & Stimulus condition & 1.80 & 2,30 & 0.1833 \\
\hline & & Stimulation & 0.21 & 3,45 & 0.8865 \\
\hline & & Stimulus condition $\times$ stimulation & 0.33 & 6,90 & 0.9215 \\
\hline & \multirow{7}{*}{$\begin{array}{l}\text { Contraversive target/, } \\
\text { ipsiversive distractor, } \\
\text { ipsiversive target/ } \\
\text { contraversive distractor }\end{array}$} & Stimulus condition & 0.02 & 1,15 & 0.8920 \\
\hline & & Difficulty & 0.03 & 1,15 & 0.8569 \\
\hline & & Stimulation & 0.43 & 3,45 & 0.7303 \\
\hline & & Stimulus condition $\times$ difficulty & 0.35 & 1,15 & 0.5610 \\
\hline & & Stimulus condition $\times$ stimulation & 1.96 & 3,45 & 0.1330 \\
\hline & & Difficulty $\times$ stimulation & 0.36 & 3,45 & 0.7808 \\
\hline & & Stimulus condition $\times$ difficulty $\times$ stimulation & 1.19 & 3,45 & 0.3248 \\
\hline \multirow{10}{*}{$\begin{array}{l}\text { No response } \\
\text { selection }\end{array}$} & \multirow{3}{*}{$\begin{array}{l}\text { Easy double distractor, } \\
\text { difficult double distractor, } \\
\text { double target }\end{array}$} & Stimulus condition & 15.22 & 2,30 & $2.74 \times 10^{-5}$ \\
\hline & & Stimulation & 1.37 & 3,45 & 0.2648 \\
\hline & & Stimulus condition $\times$ stimulation & 1.48 & 6,90 & 0.1940 \\
\hline & \multirow{7}{*}{$\begin{array}{l}\text { Contraversive target/, } \\
\text { ipsiversive distractor, } \\
\text { ipsiversive target// } \\
\text { contraversive distractor }\end{array}$} & Stimulus condition & 2.15 & 1,15 & 0.1630 \\
\hline & & Difficulty & 1.18 & 1,15 & 0.2952 \\
\hline & & Stimulation & 0.54 & 3,45 & 0.6550 \\
\hline & & Stimulus condition $\times$ difficulty & 7.10 & 1,15 & 0.0177 \\
\hline & & Stimulus condition $\times$ stimulation & 2.01 & 3,45 & 0.1254 \\
\hline & & Difficulty $\times$ stimulation & 0.78 & 3,45 & 0.5105 \\
\hline & & Stimulus condition $\times$ difficulty $\times$ stimulation & 4.49 & 3,45 & 0.0077 \\
\hline
\end{tabular}


Table 2.6 Results of repeated-measures ANOVAs on the proportions of two different error types among all trials in single-distractor and single-target conditions.

\begin{tabular}{|c|c|c|c|c|c|}
\hline Error type & Stimulus conditions & Source of variation & $F$ & $d f$ & $p$ \\
\hline \multirow{7}{*}{$\begin{array}{l}\text { Fixation } \\
\text { breaks }\end{array}$} & \multirow{7}{*}{$\begin{array}{l}\text { Single target, } \\
\text { single easy distractor, } \\
\text { single difficult distractor }\end{array}$} & Stimulus condition & 4.00 & 2,30 & 0.0289 \\
\hline & & Hemifield & 0.71 & 1,15 & 0.4124 \\
\hline & & Stimulation & 1.11 & 3,45 & 0.3566 \\
\hline & & Stimulus condition $\times$ hemifield & 0.37 & 2,30 & 0.6916 \\
\hline & & Stimulus condition $\times$ stimulation & 1.19 & 6,90 & 0.3198 \\
\hline & & Hemifield $\times$ stimulation & 2.21 & 3,45 & 0.1001 \\
\hline & & Stimulus condition $\times$ hemifield $\times$ stimulation & 0.40 & 6,90 & 0.8789 \\
\hline \multirow{7}{*}{$\begin{array}{l}\text { No response } \\
\text { selection }\end{array}$} & \multirow{7}{*}{$\begin{array}{l}\text { Single target, } \\
\text { single easy distractor, } \\
\text { single difficult distractor }\end{array}$} & Stimulus condition & 8.83 & 2,30 & $9.63 \times 10^{-4}$ \\
\hline & & Hemifield & 1.20 & 1,15 & 0.2905 \\
\hline & & Stimulation & 5.49 & 3,45 & 0.0027 \\
\hline & & Stimulus condition $\times$ hemifield & 2.87 & 2,30 & 0.0721 \\
\hline & & Stimulus condition $\times$ stimulation & 0.80 & 6,90 & 0.5734 \\
\hline & & Hemifield $\times$ stimulation & 0.44 & 3,45 & 0.7281 \\
\hline & & Stimulus condition $\times$ hemifield $\times$ stimulation & 56.00 & 6,90 & 0.7588 \\
\hline
\end{tabular}




\subsection{Discussion}

In this study we investigated the effects of unilateral electrical microstimulation of the dPul on visuomotor response selection in a visuospatial choice task in which a monkey was to discriminate potential saccade targets from distractors presented in the peripheral visual space. The animal had to make saccade responses towards targets while ignoring distractors or maintain central eye fixation when no target was present. We electrically stimulated the $\mathrm{dPul}$ at different time points relative to the go signal, which was the time the visual stimuli and thus the potential response options were presented. Pulvinar stimulation starting before the go signal resulted in a decreased number of saccades towards contraversive stimuli, i.e. stimuli presented contralateral to the stimulated hemisphere, and a higher number of saccades towards ipsiversive stimuli, i.e. stimuli presented ipsilateral to the side of stimulation. Stimulation at or after the go signal had opposite effects with a higher proportion of saccades towards contraversive stimuli and a decreased number of saccades towards ipsiversive stimuli. Stimulation only affected the proportion of fixation responses in conditions with low perceptual difficulty where targets and distractors were easy to discriminate. Strongest effects on fixation responses were observed in the condition with two easy distractors. In this condition stimulation before the go signal was associated with a decreased number of fixation responses accompanied by an increased number of saccades towards the ipsiversive distractor. Again, stimulation at or after the go signal led to opposite effects with a higher number of fixation responses and a decreased proportion of ipsiversive saccades. Due to the design of the behavioral paradigm, changes in visuomotor response selection were also associated with changes in performance depending on whether and where a target was present.

\subsubsection{Pulvinar facilitates visuomotor responses towards contralateral stimuli}

The main goal of the present study was to investigate whether the dorsal pulvinar's function is related to the filtering of visual distractors in the contralateral visual hemifield or whether it mainly serves to facilitate visuomotor responses towards contralateral visual stimuli. As mentioned above, these two functions can be considered opposing mechanisms as they lead to opposite predicted outcomes of dPul stimulation with respect to visuomotor response selection in our visuomotor choice paradigm. Assuming that stimulation potentiates the neuronal mechanisms underlying pulvinar function, if the filtering of visual distractors in the contralateral visual hemifield was the pulvinar's main function, one would expect that stimulation in our paradigm leads to an enhanced filtering of contraversive distractors. Moreover, if stimulation also activated the filtering mechanism when a target is presented on the 
contraversive side, this contraversive target would be filtered out erroneously. Additionally, the enhanced filtering of contraversive stimuli in turn may make stimuli in the opposite, ipsiversive hemifield more salient. As a result, one would predict that stimulation leads to a decreased number of both incorrect saccades towards contraversive distractors and correct saccades towards contraversive targets while the number of saccades towards ipsiversive stimuli should be increased. On the contrary, if the pulvinar was mainly involved in the facilitation of visuomotor responses towards contralateral visual stimuli, electrical stimulation of the pulvinar should generally activate responses towards contraversive stimuli resulting in an increased number of both correct saccades to contraversive targets and incorrect saccades to contraversive distractors. At the same time, stimulation should lead to a decreased number of saccades towards ipsiversive stimuli.

The effects on visuomotor response selection that we observed for dPul stimulation at or after the go signal, i.e. when the visual information and the potential response options were available, match with the predicted changes in response selection arising from the hypothesis that the $\mathrm{dPul}$ is involved in the facilitation of contralateral responses as stimulation was consistently associated with a decreased number of saccades towards both ipsiversive targets and distractors. In some conditions the decrease in the number of ipsiversive saccades was accompanied by an increase in the number of saccades towards both contraversive targets and distractors. Thus, we did not find evidence that the dPul may be involved in the filtering of contraversive distractors.

Our results resemble the effects of pulvinar stimulation on saccade choices found by Dominguez-Vargas et al. (2017). Similar to our findings in conditions with two peripheral stimuli, in their study dPul stimulation starting at and after the go signal led to an increased number of contraversive choices in a free-choice saccade task with equally-rewarded bilateral targets. In contrast, stimulation starting before the go signal was associated with an increase in the number of ipsiversive choices. The effect of stimulation starting before the go signal was also found consistently in the present study. More specifically, we found a consistent increase in the number of saccades towards ipsiversive stimuli even when the ipsiversive stimulus was a distractor that was easily distinguishable from a target. That is, unlike in the free-choice paradigm used in Dominguez-Vargas et al. (2017), in conditions with an ipsiversive distractor the execution of this ipsiversive response did not even yield a reward. Thus, our data support the authors' conclusion that unilateral pulvinar stimulation induces an overall contraversive drive activating responses to contraversive stimuli. This contraversive drive may even account for the increase in ipsiversive responses found for stimulation starting before the go signal as in this condition stimulation starts before the presentation of the visual stimuli, that is, when the animal is still required to maintain central eye fixation. In order 
to do so, the animal might need to counteract the stimulation-induced contraversive drive by activating a putative compensatory ipsiversive mechanism which might be engaged until the motor planning/execution phase of the task resulting in the observed higher number of ipsiversive choices. The effects of dPul stimulation starting before the go signal were even independent of the difficulty of the perceptual discrimination between targets and distractors. This finding may suggest that pulvinar stimulation, if delivered early enough before visual stimulus onset, leads to the activation of a compensatory signal related to an ipsiversive saccadic response that has already reached a stage close to motor execution when the visual information becomes available. Hence, this compensatory ipsiversive motor plan cannot be overridden by the signal arising from the actual ipsiversive stimulus even if that stimulus clearly indicates a different response than the response activated through the compensatory ipsiversive motor plan.

When the pulvinar is stimulated at or after the go signal, stimulation only starts when or after the visual information has become available and the animal is supposed to give a response. In this case, the stimulation-induced contraversive drive may lead to an imbalance in the competition between the two stimulus-driven signals, eventually favoring the contraversive response. However, in the current study, a decrease in the number of ipsiversive saccades was only accompanied by an increase in contraversive saccades when targets and distractors were difficult to discriminate from each other. That is, the behavioral outcome of the stimulation-induced contraversive drive might depend on the strength of the signal driven by the contraversive stimulus. In our task design, in conditions with high perceptual similarity between a contraversive distractor and an ipsiversive target, the difficult contraversive distractor may already activate a contraversive saccade response which is further enhanced by pulvinar stimulation resulting in a higher number of (erroneous) contraversive saccades. However, when the contraversive distractor and the ipsiversive target can be easily distinguished from each other, the stimulation-induced contraversive drive may still prevent the planning and execution of an ipsiversive saccade but the "stop" signal driven by the easy contraversive distractor may be strong enough to compensate for the stimulation-induced contraversive drive. As a result, the animal selects the third response option, i.e. the fixation response, more often.

\subsubsection{Pulvinar plays a general role in resolving visuomotor response competition}

Previous research on monkeys (Desimone et al., 1990; Dominguez-Vargas et al., 2017) and patients with pulvinar lesions (Danziger et al., 2004; Ward \& Danziger, 2005) suggested 
that the pulvinar is most strongly involved in contralateral response activation when the contralateral response must be activated in preference to a competing ipsilateral response, i.e. under conditions of response competition. Importantly, all studies mentioned above investigated the relevance of response competition by presenting peripheral visual stimuli in opposite hemifields, with each peripheral stimulus being linked to a particular visuomotor response. In the present study, we wanted to investigate whether the pulvinar is only involved in resolving competition between responses linked to peripheral visual stimuli or whether the pulvinar plays a more general role in resolving visuomotor response competition. To this end, our task design always included the additional response option to withhold a saccadic response and maintain central eye fixation at the cenrally presented fixation spot. Thus, even when only one peripheral stimulus was presented there was competition between two visuomotor response options. We found consistent effects of dPul stimulation on visuomotor response selection in conditions with a single ipsiversive stimulus, that is, when the animal was to choose between an ipsiversive saccade response and maintaining fixation. When the ipsiversive stimulus was a distractor, stimulation before the go signal led to an increased number of incorrect ipsiversive saccades towards the distractor whereas stimulation at or after the go signal was associated with a decreased number of ipsiversive saccades. The decrease in the number of ipsiversive saccades when stimulation started after the go signal was also found in trials with a single ipsiversive target. Note that in these conditions the saccade response and the fixation response are complementary. That is, an increase in saccade responses is always associated with a decrease in fixation responses and vice versa.

Overall, the effects of pulvinar stimulation on ipsiversive saccade responses in conditions with a single peripheral stimulus are similar to the results found for conditions with two peripheral stimuli. These findings suggest that the pulvinar plays a more general role in resolving competition between competing visuomotor response options even when there is only one potential saccade target available. Moreover, the effects may also be explained by a stimulation-induced contraversive drive. Similar to conditions with two peripheral stimuli, the increase in the number of saccades towards single, ipsiversive distractors when stimulation started before the go signal might result from a compensatory ipsiversive mechanism counteracting the stimulation-induced contraversive drive. Similarly, the decrease in the number of saccades towards single, ipsiversive stimuli found for stimulation at or after the go signal may be explained by a stimulation-induced contraversive drive preventing the planning and execution of an ipsiversive saccade. Hence, the animal selects the fixation response more frequently.

Our results on the role of the $\mathrm{dPul}$ in resolving visuomotor response competition are also in line with Findlay \& Walker's (1999) model of saccade generation proposing a competitive 
push-pull interaction between a fixate center and a move center. According to this model, saccadic movements are only generated when the activity in the fixate center falls below a certain threshold and a trigger signal opens a gate at the level of a motor command. Due to reciprocal inhibitory connections between the fixate and the move center an increase in move center activity will reduce activity in the fixate center and thus promote disengagement, i.e. saccade generation. In addition, multiple potential saccade goals compete within a salience map represented in the move center in a winner-take-all fashion through inhibitory cross-links between the various regions of the map. That is, the location of the peak in the salience map will be selected as the unique saccade goal. However, fixate center activity can also be influenced directly by fixation and peripheral visual events as well as by automated higher-level processes such as intrinsic salience and spatial selection. The various competing information signals are then integrated to decide whether and where a saccade should be made.

The current findings can be embedded in this framework of a fixate/move balance. First, the go signal, which was the dimming of the fixation spot (fixation visual event) and the onset of one or two peripheral visual stimuli (peripheral visual event), might have led to a reduction in fixate center activity changing the fixate/move equilibrium at the level of automatic direct visual influences eventually promoting saccade generation. However, there may be a difference between the competition occurring in conditions with a single peripheral visual stimulus and conditions with two peripheral stimuli. In single-stimulus conditions, there should only be competition between the fixate and the move center, that is, between the option to make a saccade towards the peripheral stimulus and the option to maintain eye fixation. In contrast, in conditions with two peripheral stimuli, additional competition should arise within the salience map of the move center because two potential saccade goals are available. As discussed before, the competition between the fixate and the move center in single-stimulus conditions was enough to engage the contralateral response facilitation mechanism of the pulvinar so that pulvinar stimulation at or after the go signal might have led to a fixate/move imbalance. In conditions with two peripheral stimuli a spatial selection had to be made in addition to the decision between fixation and movement. In this case, pulvinar stimulation might not only affect the fixate/move equilibrium but might also influence the location of the peak in the salience map, for instance, by potentiating the salience of contraversive stimuli. Therefore, the stimulation-induced contraversive drive might reflect both the promoting of disengagement and a change of the peak location in the salience map.

When stimulation starts before the go signal the compensatory ipsiversive signal activated to counteract the stimulation-induced contraversive drive and the go signal itself may be strong enough to inhibit fixate center activity to a value below threshold opening the gate to 
motor execution immediately at the time of the go signal. The idea of push-pull interactions between activations from different visual objects in multiple brain systems is also suggested by the integrated competition hypothesis of visual attention (Duncan et al., 1997). Therefore, the influence of pulvinar stimulation on activity in the sensorimotor system elicited by competing visual objects may also reflect changes in visuospatial attention such that the focus of attention is shifted towards the selected object.

\subsubsection{Pulvinar microstimulation effects on response selection do not only reflect changes in motor execution}

The lack of a three-way interaction between stimulus condition, hemifield, and stimulation in all ANOVAs applied on saccade reaction times showed that pulvinar stimulation affected the latencies of both correct ipsiversive and correct contraversive saccades in a very similar way across stimulus conditions. More specifically, stimulation before the go signal consistently facilitated correct saccades to ipsiversive targets in all stimulus conditions and, in addition, led to shorter latencies of correct contraversive saccades in single-target and easy targetdistractor trials. Stimulation at the go signal led to longer latencies of saccades to both sides in double-target and difficult target-distractor trials and also delayed ipsiversive saccades in single-target and easy target-distractor trials. Finally, stimulation after the go signal led to a consistent delay of correct saccades to both sides in all stimulus conditions. Therefore, it is unlikely that the effects of pulvinar stimulation on response selection only reflect effects on pure motor execution. This conclusion is further supported by the finding that changes in response selection were different between conditions although saccade latencies were affected in a very similar way. For instance, stimulation after the go signal led to increased latencies of correct saccades in both double-target and difficult target-distractor conditions but response selection was only affected in the latter conditions. Thus, pulvinar stimulation effects on saccade latencies cannot fully explain changes in response selection. Moreover, if stimulation effects mainly reflected motor effects, one would expect to observe a very similar pattern of response selection across all visual stimulus conditions. This was not the case in our study as the way how stimulation-induced changes in the proportion of ipsiversive saccades were compensated at the behavioral level depended on the perceptual similarity between the contraversive stimulus and the target. Additionally, at the beginning of each stimulation session it was ensured that stimulation did not evoke reflexive saccades and stimulation had no effect on the occurrence of errors that were not related to response selection but may occur due to effects on motor execution (fixation breaks and no valid response selection). Additional evidence against purely motor-related effects of modulations in pulvinar activity 
comes from a study on patients with pulvinar lesions that showed an impaired ability to make saccades towards contralesional targets in a temporal-order judgment task but were impaired in initiating saccades away from contralesional targets in an antisaccade task (Arend et al., 2008). This finding supports the conclusion that changes in pulvinar activity generally affect visuomotor actions linked to visual stimuli in the hemifield contralateral to the side of interference.

\subsubsection{Alternative explanations and limitations}

A previous study using reversible pharmacological inactivation of the macaque $\mathrm{dPul}$ in a visuomotor reward-modulation task found that the strong inactivation-induced bias in saccade choices towards the ipsilesional hemifield could be alleviated if the stimulus in the contralesional hemifield was associated with a higher reward (Wilke et al., 2013). That is, effects of changes in pulvinar activity on spatial choice can be compensated by differences in the expected reward associated with the potential choice options. Similarly, an interaction of expected reward with electrical microstimulation was found for perceptual decisions biased by stimulation of area MT (Cicmil et al., 2015). Our visuomotor choice paradigm may also be considered a reward-modulation paradigm as saccades to targets always yielded a reward whereas saccades towards distractors did not yield a reward. In the framework of competition between a fixate center and a move center in the brain (Findlay \& Walker, 1999) the different reward signals driven by targets and distractors in our task design might influence the intrinsic salience of the peripheral stimuli such that targets are highly intrinsically salient whereas distractors, particularly easy distractors have a low intrinsic salience. Hence, not only the strength of the signal directly linked to the visuomotor action driven by the contraversive stimulus (to make a saccade or not) might determine the behavioral outcome of the stimulation-induced contraversive drive but also the strength of the reward signal associated with that stimulus. For instance, in the condition with an ipsiversive target and an easy contraversive distractor the stimulation-induced contraversive drive may still hamper the planning and execution of an ipsiversive saccade towards the target but the reason why the animal does not make a saccade towards the contraversive distractor but chooses to maintain fixation more often might be that due to the low reward signal driven by the distractor the intrinsic salience of this contraversive stimulus is still too low to activate the move center to an extent that would make fixate center activity fall below the threshold to promote disengagement, that is, the execution of a contraversive saccade. However, this interpretation only applies to pulvinar stimulation starting at or after the go signal when the visual stimuli and thus reward information are available at the time stimulation starts and cannot account for the effects of stimulation starting before the go signal. 
Furthermore, the delay in saccadic reaction times when pulvinar stimulation started at or after the go signal could also be interpreted as a disruptive effect on normal pulvinar function in terms of "neural hijacking" as it was suggested for high-frequency electrical stimulation in cortex (Cheney et al., 2013; Griffin et al., 2011). However, disruption of pulvinar function can hardly explain the consistent decrease in the number of saccades towards ipsiversive stimuli and the increase in the number of contraversive responses that we observed for pulvinar stimulation at or after the go signal because one would predict the opposite behavioral outcome if the activation of contraversive responses was hampered. Moreover, execution of contraversive and ipsiversive saccades was affected in a quite similar way. That is, an imbalance between the extent of disruption of contraversive and ipsiversive responses cannot explain the effects of pulvinar stimulation on response selection in our study (Dominguez-Vargas et al., 2017). In addition, the disruption of a putative mechanism for filtering contraversive distractors may explain our results for response selection in conditions with contraversive distractors since the disrupted filtering of contraversive distractors should lead to a higher number of responses towards these distractors accompanied by a lower number of ipsiversive responses. However, the disruption of such a filtering mechanism cannot explain the effects of pulvinar stimulation on response selection in conditions with contraversive targets because target stimuli should not even activate such a filtering mechanism but rather inhibit filtering of contralateral stimuli. If pulvinar stimulation disrupted this inhibitory effect of target stimuli, one would expect an erroneous filtering of contraversive targets resulting in a decreased number of contraversive responses and an increased number of ipsiversive responses. Our data, however, show the opposite effect and were fairly consistent across conditions independent of the type of stimulus presented in the contraversive hemifield.

In contrast to Dominguez-Vargas et al. (2017), we do not find stimulation effects on visuomotor response selection in the double-target condition. If the interaction between the stimulation-induced contraversive drive and the visuomotor signal driven by the contraversive stimulus determined visuomotor response selection, one would expect an increase in contraversive saccades when the animal is presented with two peripheral targets and stimulation starts after the go signal as both stimulation and the contraversive target should drive a contraversive saccade response. Compared to Dominguez-Vargas et al. (2017) in our paradigm reaction times were generally longer shifting the beginning of stimulation relative to the time of the visuomotor decision and movement initiation. Thus, stimulation starting later than $80 \mathrm{~ms}$ after the go signal might resemble Dominguez-Vargas et al.'s (2017) condition with late stimulation starting $80 \mathrm{~ms}$ after the go signal more appropriately. Furthermore, in the current study single- and double-target trials were randomly interleaved with conditions where a distractor could be present whereas in Dominguez-Vargas et al. (2017) each visual 
stimulus represented a saccade target. Hence, the general task context might also influence how pulvinar stimulation affects visuomotor response selection in free-choice trials.

\subsubsection{Conclusions}

In our study dPul stimulation affected visuomotor response selection in a time-dependent manner: stimulation starting before the go signal, which at the same time was the time of the onset of the peripheral visual stimuli, led to a consistent increase in the number of ipsiversive saccade choices even when the ipsiversive stimulus the saccade was directed to was a distractor that was easily distinguishable from a target. In contrast, stimulation starting at or after the go signal was associated with a consistent decrease in the number of ipsiversive saccade choices. These results are consistent with the hypothesis that the dPul facilitates visuomotor responses to visual stimuli present in the contralateral hemifield and thus do not support evidence suggesting that the pulvinar is mainly involved in the filtering of contralateral visual distractors.

Interestingly, the changes in ipsiversive response selection were only accompanied by complementary changes in contraversive saccade choices when the contraversive stimulus was a target or a stimulus that was difficult to discriminate from a target. When the contraversive stimulus was a distractor that was easy to discriminate from a target the changes in ipsiversive saccade choices were accompanied by complementary changes in fixation responses suggesting that the behavioral outcome of pulvinar microstimulation is determined by an interaction of a stimulation-induced contraversive drive and the visuomotor signal driven by the contraversive stimulus. In addition, pulvinar microstimulation even affected ipsiversive saccade choices when only one peripheral stimulus indicating a potential saccade response was presented with the response option to maintain fixation indicating that the pulvinar does not only play a role in resolving visuomotor response competition driven by peripheral visual stimuli but plays a more general role in resolving competition between multiple visuomotor response options. Of course, future research should test the reliability of the current results in a second animal.

\section{Acknowledgments}

We thank Uwe Zimmermann for collecting a subset of the data and for providing analysis code, Dr. Sebastian Möller for sharing the design of the electrode drive, Lukas Schneider for support in programming the task controller, and Ira Panolias, Sina Plümer, Leonore Burchardt, Klaus Heisig, and Dirk Prüße for technical support. 



\section{Chapter 3}

\section{Effects of pulvinar and LIP microstimulation on whole-brain space representations}

\subsection{Introduction}

The dorsal pulvinar ( $\mathrm{dPul}$ ), the largest thalamic nucleus, and the lateral intraparietal area (LIP), which is located in the intraparietal sulcus (IPS) of the posterior parietal cortex (PPC), share strong reciprocal anatomical connections (Asanuma et al., 1985; Blatt et al., 1990; Hardy \& Lynch, 1992; Romanski et al., 1997). Moreover, they have a similar cortical connectivity with both regions being anatomically connected to other areas in PPC (Andersen et al., 1990a; Asanuma et al., 1985; Blatt et al., 1990), the lower and upper bank of the superior temporal sulcus (STS) (Blatt et al., 1990; Yeterian \& Pandya, 1989, 1991; Zhong \& Rockland, 2003), areas in prefrontal cortex (Asanuma et al., 1985; Blatt et al., 1990; Romanski et al., 1997; Trojanowski \& Jacobson, 1976), occipito-temporal cortex (Andersen et al., 1990a; Webster et al., 1993, 1995; Zhong \& Rockland, 2003), and extrastriate visual cortex (Andersen et al., 1990a; Blatt et al., 1990; Shipp, 2001; Ungerleider et al., 2008, 2014).

$\mathrm{dPul}$ and LIP also share functional properties of visuomotor processing. Both regions have visually responsive neurons (Andersen et al., 1990b; Colby et al., 1996; DominguezVargas et al., 2017; Platt \& Glimcher, 1998; Robinson et al., 1986) and a homogenous distribution of receptive fields located in the contralateral or ipsilateral hemifield and varying in size (Benevento \& Miller, 1981; Blatt et al., 1990; Platt \& Glimcher, 1998). However, the receptive fields of both sites appear to be usually centered in the contralateral hemifield 
(Andersen et al., 1990a; Ben Hamed et al., 2001; Benevento \& Miller, 1981; Blatt et al., 1990). Moreover, cells in both regions respond more strongly to a visual stimulus when it serves as a saccade target than when the same stimulus is presented during passive fixation (Petersen et al., 1987) or as a visual distractor (Platt \& Glimcher, 1997; Suzuki \& Gottlieb, 2013), most likely reflecting modulation of neuronal responses by visuospatial attention (Bender \& Youakim, 2001; Bisley \& Goldberg, 2003; Colby et al., 1996; Petersen et al., 1985, 1987). Additionally, both dPul and LIP neurons show saccade-related activity (Acuna et al., 1983; Andersen et al., 1990b; Dominguez-Vargas et al., 2017; Platt \& Glimcher, 1998; Premereur et al., 2011; Robinson et al., 1986) and signals related to other higher cognitive processes such as perceptual decision-making (Katz et al., 2016; Komura et al., 2013). Furthermore, pharmacological inactivation of both LIP and dPul leads to a bias in saccadic choice behavior towards the visual hemifield ipsilateral to the side of inactivation (Wilke et al., 2012, 2013, 2010) whereas electrical microstimulation of both regions affects saccadic choices in the opposite direction with higher proportions of choices towards stimuli in the hemifield contralateral to the side of stimulation (Dai et al., 2014; Dominguez-Vargas et al., 2017). In addition, both dPul and LIP microstimulation can evoke saccades into the contralateral hemifield (Dominguez-Vargas et al., 2017; Thier \& Andersen, 1998)

Given these similarities in anatomical connectivity and functional properties of dPul and LIP, we investigated the "effective connectivity" (Matsui et al., 2011) of both dPul and LIP using a combination of functional magnetic resonance imaging (fMRI) and electrical microstimulation in awake rhesus monkeys in order to identify the predominant functional pathways shared by dPul and LIP. The combination of fMRI and electrical microstimulation has been shown to be a useful method to study functional networks of cortical (Moeller et al., 2008; Premereur et al., 2016; Sultan et al., 2011; Tolias et al., 2005) and subcortical (Field et al., 2008; Logothetis et al., 2010) brain regions in both anesthetized and awake monkeys (Logothetis et al., 2010) and it is a suitable technique for the direct comparison of the functional connectivity of different brain areas (Petkov et al., 2015). Interestingly, even different stimulation sites located close to each other within an area in IPS reveal distinct networks (Premereur et al., 2015b). In order to identify potentially distinct effective connectivity patterns within brain regions in our study we stimulated two different sites in dPul and LIP, respectively.

Distinct modulations of brain networks, including activity in area LIP, could also be found in studies using fMRI in alert monkeys performing visually-guided saccades or arm movements (Premereur et al., 2015a) or covert shifts of spatial attention (Caspari et al., 2015). fMRI activity in LIP was also found to be modulated by saccade direction (Koyama et al., 2004). Moreover, when monkeys perform memory-guided saccades fMRI activation can 
be found in a functional network consisting of brain areas located in the arcuate sulcus and the principal sulcus of frontal cortex, along IPS in the parietal cortex including LIP, and areas in STS (Kagan et al., 2010), partially overlapping with a frontoparietal functional network preferentially activated by saccadic eye movements (Premereur et al., 2015a). Pharmacological inactivation of LIP during the performance of memory-guided saccades leads to a reduction in fMRI activation in frontal and temporal regions of that network. Notably, this modulation of activity is task-specific with a stronger reduction of fMRI signal in contralesional trials, that is, when the animals are presented with visual cues and remember spatial locations in the hemifield contralateral to the side of inactivation (Wilke et al., 2012). Similar effects result from reversible inactivation of dPul. Again, when monkeys perform memory-guided saccades fMRI activity in several frontoparietal and parietotemporal regions, which are part of the functional saccade network, is reduced in a task-specific manner with a more pronounced reduction of activation for contralesional trials compared to ipsilesional trials (Wilke et al., in revision). The effects of electrical microstimulation on fMRI brain signals were also shown to be dependent on task variables. Ekstrom et al. (2008) found a topographically specific pattern of enhancement and suppression of fMRI activity in early visual areas following microstimulation of the frontal eye field (FEF) only in the presence of visual input. Another study combining fMRI and FEF microstimulation found more pronounced modulations in fMRI activity with increasing task demands (Premereur et al., 2013). Given (1) the activation of a distinct functional network during the performance of visually- and memory-guided saccades, consisting of frontal, parietal, and temporal brain regions, (2) the task specificity of perturbation effects on fMRI activations of this network in alert monkeys, and (3) the high similarity between the changes in brain fMRI signals resulting from LIP and dPul inactivation, we hypothesized that electrical microstimulation of both dPul and LIP would alter fMRI activity in frontal, parietal, and temporal cortical areas in a task-specific manner. To test this hypothesis, we had two rhesus monkeys perform a memory-guided saccade task while either dPul or LIP were electrically stimulated in the memory epoch of the task in an event-related fMRI design.

\subsection{Methods}

\subsubsection{Procedures}

All experimental procedures were conducted in accordance with the European Directive 2010/63/EU, the corresponding German law governing animal welfare, and German Primate Center institutional guidelines. The procedures were approved by the responsible government 
agency (Niedersaechsisches Landesamt fuer Verbraucherschutz und Lebensmittelsicherheit (LAVES), Oldenburg, Germany).

\subsubsection{Animal preparation}

Two male rhesus monkeys (Macaca mulatta), weighing $9 \mathrm{~kg}$ and $10.5 \mathrm{~kg}$, respectively, served as subjects. Surgical procedures were similar for both animals. In an initial surgery, each animal was implanted with an MRI-compatible polyetheretherketone (PEEK) head post embedded in a bone cement head cap (Palacos with gentamicin; BioMet) anchored by ceramic screws (Rogue Research) under general anesthesia and aseptic conditions. MRvisible markers were embedded in the head cap to aid the planning of the chamber in stereotaxic space (Monkey C, right hemisphere: center at 0.5 A/14.5 R mm, tilted -11 P/27 R degrees; Monkey B, right hemisphere: center at 3.5 P/17 R mm, tilted 15 P/30 R degrees) with the MR-guided stereotaxic navigation software Planner (Ohayon \& Tsao, 2012). A separate surgery was performed in each animal to implant a PEEK MRI-compatible chamber (inside diameter $22 \mathrm{~mm}$ ) allowing access to the right pulvinar and LIP. After confirming chamber positioning with postsurgical MRI measurements, a partial craniotomy was made inside the chamber of each animal.

\subsubsection{Electrical microstimulation}

An S88X dual output square pulse stimulator (Grass Products, Natus Neurology, USA) triggered by a MATLAB-based task controller generated $200 \mathrm{~ms}$ trains of twin pulses at $300 \mathrm{~Hz}$, which in turn triggered a constant current stimulus isolator A365 (World Precision Instruments, USA) to produce 60 biphasic pulses. The current (100-250 $\mu \mathrm{A})$ was delivered to the target structure using single monopolar electrodes (platinum-iridium, $100 \mathrm{~mm}$ length, $125 \mu \mathrm{m}$ thick core, initial $2 \mathrm{~cm}$ glass coating with an exposed tip of $40 \mu \mathrm{m}$, total thickness of $230 \mu \mathrm{m}$ including polyamide tubing coating, customer part ID: UEIK1, FHC Inc., USA). A return (reference) tungsten rod was placed in the chamber filled with saline. Voltage drop was monitored as the difference between voltage measured before and after a $10 \mathrm{k} \Omega$ resistor in series with the electrode using a 4 channel 1GS/s Tektronix TDS2004C oscilloscope. The manufacturer-specified impedance of the electrodes was $300-500 \mathrm{k} \Omega$. The initial impedance measured at $1000 \mathrm{~Hz}$ before the experiment was $110-1400 \mathrm{k} \Omega$. Since the impedance dropped dramatically after a few stimulation trains were applied, before each session 20-30 pulse trains were delivered to the electrode immersed in saline using $250 \mu \mathrm{A}$ current, in order to bring the electrode impedance to a more stable regime. Following this procedure, the impedance ranged from $10 \mathrm{k} \Omega$ to $70 \mathrm{k} \Omega$ in monkey $\mathrm{C}$ and from $10 \mathrm{k} \Omega$ to $120 \mathrm{k} \Omega$ in monkey 
B. In each stimulation trial, 10 consecutive current pulse trains were applied at a frequency of $1 \mathrm{~Hz}$ (see section 3.2.6 for behavioral task).

\subsubsection{MR imaging}

Both animals were scanned in a 3 T MRI scanner (Monkey C: Magnetom Tim Trio; Siemens, Monkey B: Magnetom Prisma; Siemens). For planning of chamber and electrode positioning for each animal, high-resolution full-head T1-weighted (3D magnetization-prepared rapid gradient-echo, MPRAGE, $0.5 \mathrm{~mm}$ isometric) and additional T2-weighted (rapid acquisition with relaxation enhancement, RARE, $0.25 \mathrm{~mm}$ in plane, $1 \mathrm{~mm}$ slice thickness) images with the slice package aligned to the chamber vertical axis were acquired before and after chamber implantation in an awake state or under anesthesia using the built-in gradient body transmit coil and a custom single-loop receive coil (Windmiller Kolster Scientific). T1- and T2-weighted scans were coregistered and transformed into "chamber normal" orientation (aligned to the chamber vertical axis) and into anterior commissure-posterior commissure (AC-PC) space for electrode targeting and visualization. These images were acquired with the chamber and the grid filled with gadolinium (Magnevist; Bayer)/saline solution (proportion 1:200) with tungsten rods inserted in predefined grid locations for alignment purposes.

In each experimental session, advanced interactive 3D shimming was performed. Then, T2-weighted images with the slice package aligned to the chamber vertical axis (32 slices, see above) were acquired. The first image was acquired with the electrode inserted into the brain but before placing it in the targeted brain region. Based on that image, we measured the 2D-distance between the electrode tip and the center of the target structure and adjusted the electrode position accordingly. The second image was acquired after adjusting the electrode position to confirm proper placement within the target structure allowing full control over electrode positioning. Blood-oxygen-level dependent (BOLD) functional images were acquired with a T2*-weighted echo-planar imaging (EPI) single-shot sequence (TR: $2 \mathrm{~s}$, TE: $27 \mathrm{~ms}$, flip angle: 76 , bandwidth: $1302 \mathrm{~Hz} /$ pixel, 80x80 matrix, field of view: $96 \mathrm{~mm}$, $1.2 \mathrm{~mm}$ x $1.2 \mathrm{~mm}$ in-plane resolution, 30 slices in monkey $\mathrm{C}$ and 32 slices in monkey $\mathrm{B}$, slice thickness: $1.2 \mathrm{~mm}$, inter slice time: $66.7 \mathrm{~ms}$ for monkey $\mathrm{C}$ and $61.8 \mathrm{~ms}$ for monkey $\mathrm{B}$, data acquired in separate, consecutive runs of 454 volumes each) using a custom-made 4channel receive coil and transmit coil (Windmiller Kolster Scientific). Before functional data acquisition, the received signal was checked for noise using the same echo-planar imaging sequence with the RF pulse amplitude set to zero. In addition, an in-plane anatomical image was acquired (T2-weighted, Turbo-Spin-Echo sequence, TR: 5 s, TE: $18 \mathrm{~ms}$, flip angle: $180^{\circ}$, 
$0.75 \mathrm{~mm} \times 0.75 \mathrm{~mm}$ in-plane resolution, slice thickness: $1.2 \mathrm{~mm}$ ) for later coregistration with the functional images.

\subsubsection{Pulvinar and LIP targeting}

A custom-made MR-compatible polyetherimide (Ultem) grid ( $0.8 \mathrm{~mm}$ hole spacing, 0.45 $\mathrm{mm}$ hole diameter) and a custom-made plastic XYZ manipulator drive (design courtesy of Dr. Sebastian Moeller (Moeller et al., 2008)) were used to position platinum-iridium electrodes (FHC, see section 3.2.3 for detailed specifications) in the corresponding grid hole. Grid hole determination was based on anatomical MRI using Planner (Ohayon \& Tsao, 2012) and BrainVoyager (Version 2.4.2.2070, 64-bit; Brain Innovation BV). During penetration, the
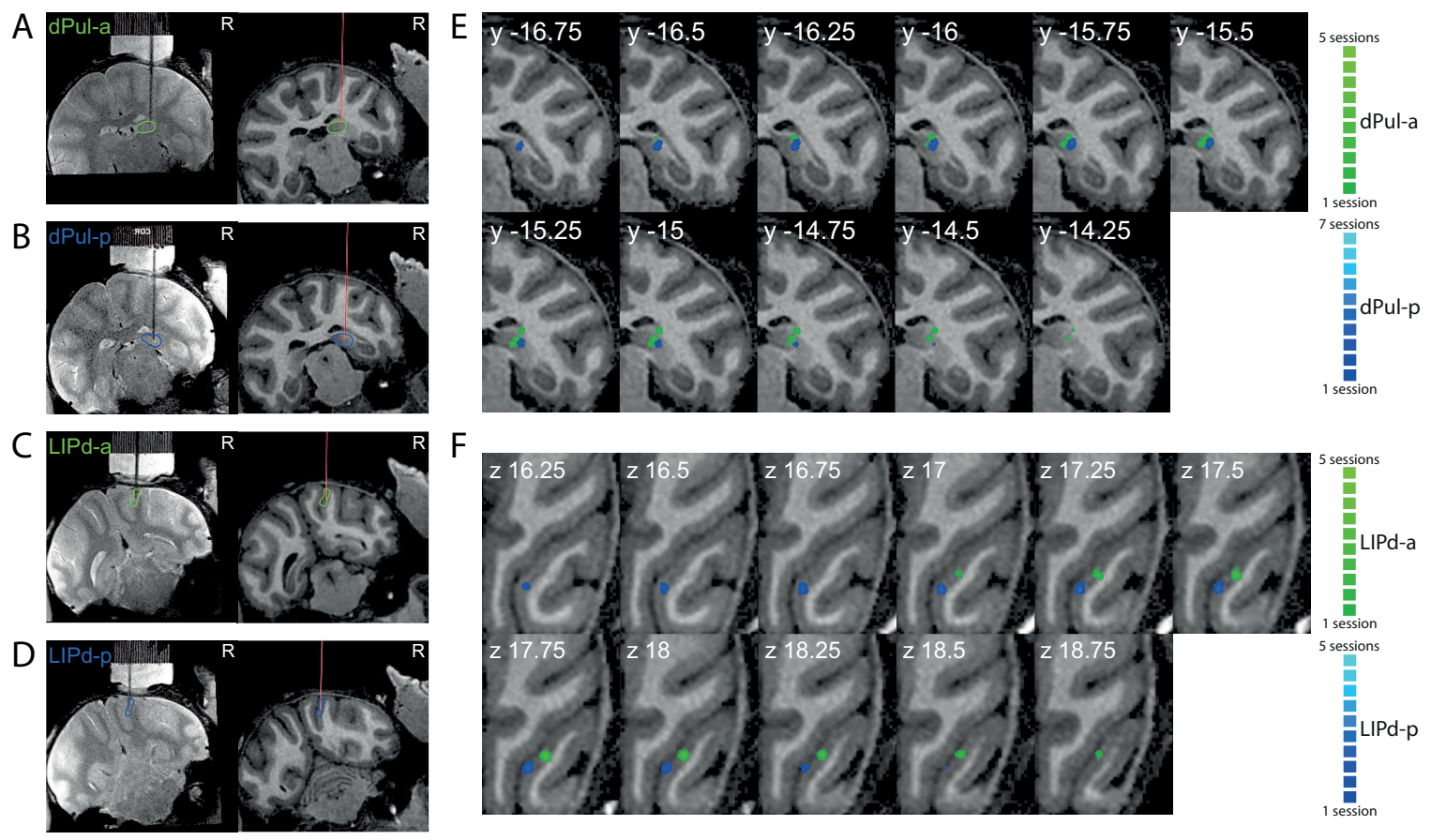

Fig. 3.1 Electrode localization in dPul and LIPd in monkey C. Electrode positions measured in T2-weighted MR images (left) and reconstructed in T1-weighted images (right), both aligned to the chamber vertical axis, for example sessions with microstimulation in (A) dPul-a,(B) dPul-p, (C) LIPd-a, and (D) LIPd-p. Green and blue outlines mark the respective target region. Probability maps of electrode positions across sessions with microstimulation in (E) dPul (green: dPul-a, blue: dPul-p) and (F) LIPd (green: LIPd-a, blue: LIPd-p) in the right hemisphere displayed on a T1-weighted MR image aligned to standard AC-PC space. dPul-a: anterior dorsal pulvinar, dPul-p: posterior dorsal pulvinar, LIPd-a: anterior dorsal lateral intraparietal area, LIPd-p: posterior dorsal lateral intraparietal area, R: right, Y: distance from AC-PC origin in the anterior/posterior plane in millimeters, $\mathrm{Z}$ : distance from $\mathrm{AC}-\mathrm{PC}$ origin in the dorsal/ventral plane in millimeters. 

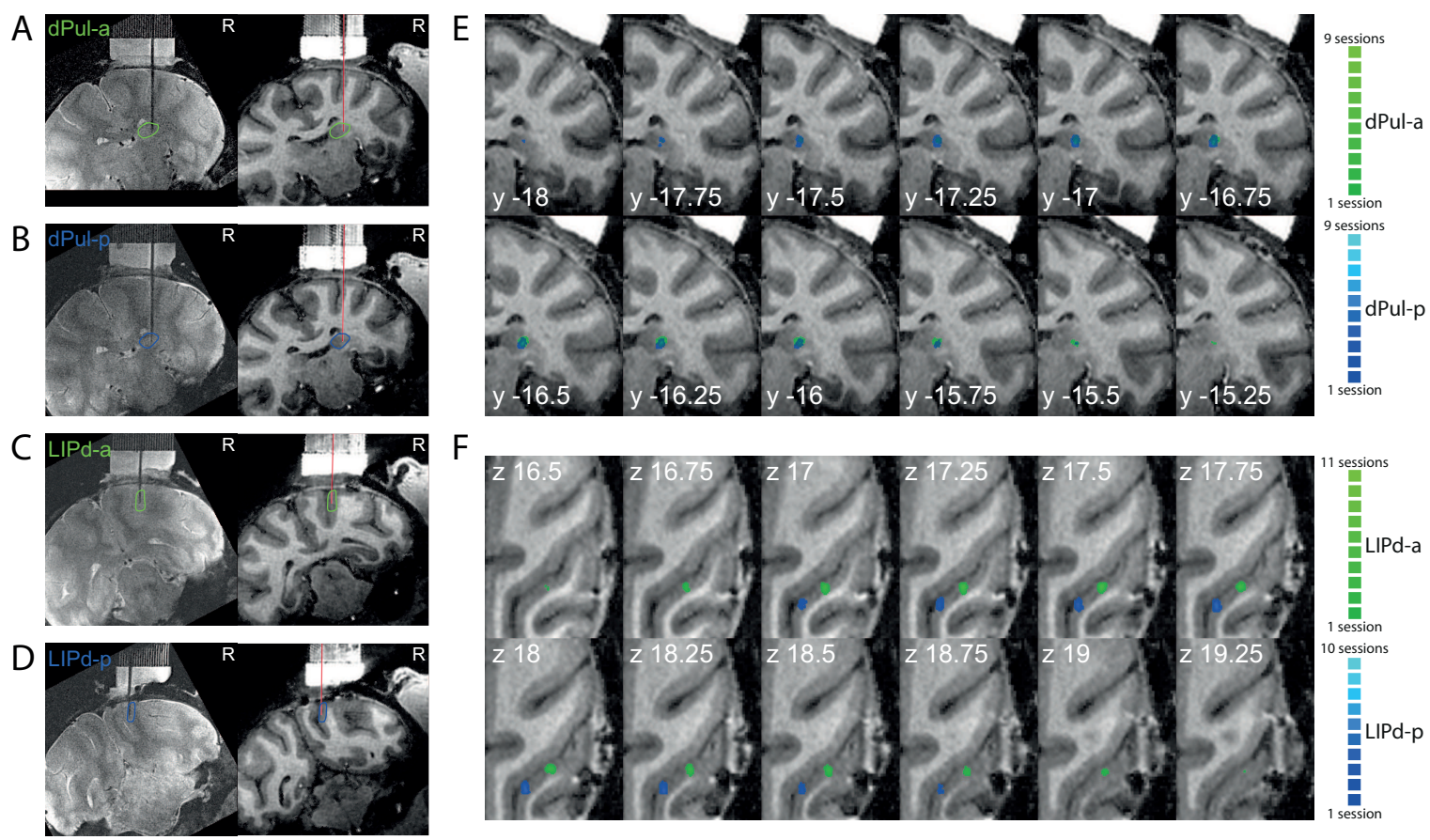

Fig. 3.2 Electrode localization in dPul and LIPd in the right hemisphere in monkey B. Same conventions as in Fig. 3.1.

electrode was protected by a custom-made MR compatible guide tube (polyimide coated fused silica, $430 \mu \mathrm{m}$ outer diameter, $320 \mu \mathrm{m}$ inner diameter; Polymicro Technologies). An MR compatible stopper (polyimide coated fused silica, $700 \mu \mathrm{m}$ outer diameter, $530 \mu \mathrm{m}$ inner diameter; Polymicro Technologies) ensured that the guide tube only penetrated the dura and minimally the cortex below. Before penetration, the electrode tip was aligned to the guide tube tip and was held in place by a drop of melted petroleum jelly. For each experimental session, the final electrode location was determined based on anatomical MRI (see section 3.2.4). In each animal, we stimulated two different sites, one located more anterior and the other located more posterior, in the right dPul and dorsal LIP, respectively, with a current strength of 250 $\mu \mathrm{A}$. For localization of the pulvinar stimulation sites we used the traditional segregation of the pulvinar nucleus into PM, PL, and PI as used in currently online available and downloadable atlases (Calabrese et al., 2015; Rohlfing et al., 2012). Fig. 3.1A-B and Fig. 3.2A-B show the electrode positions in example sessions of dPul stimulation measured in T2-weighted MR images with the slice package aligned to the chamber vertical axis and reconstructed in T1-weighted MR images for each animal, respectively (see also section 3.2.4). Fig. 3.1E and Fig. 3.2E show probability maps of the estimated electrode tip positions across dPul stimulation sessions for both animals, respectively (see section 3.2.7 for a detailed description of the generation of the displayed probability maps). As can be seen in Fig. 3.1 and Fig. 3.2, 


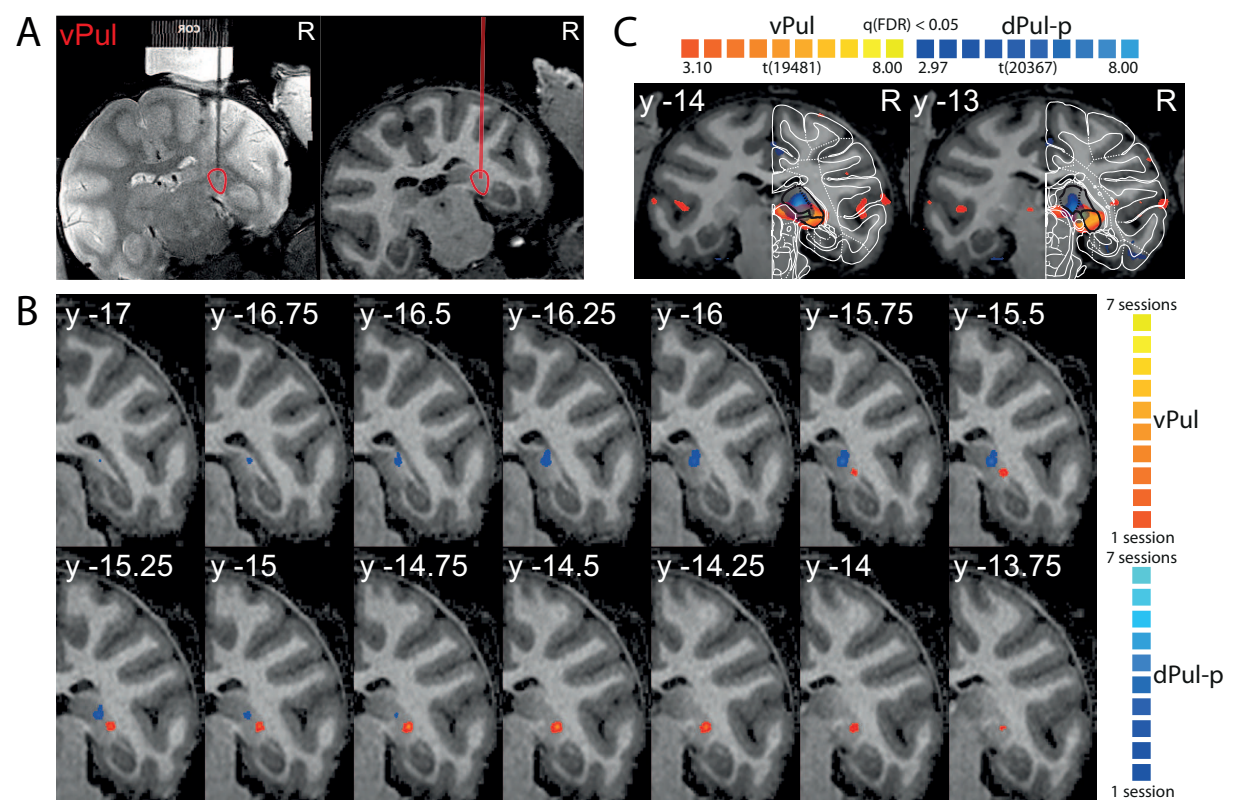

Fig. 3.3 Electrode localization for low-current stimulation in dPul-p and vPul in monkey C. (A) Electrode position measured in a T2-weighted MR image (left) and reconstructed in a T1-weighted image (right), both aligned to the chamber vertical axis, for an example session with vPul stimulation. (B) Probability maps of electrode positions across sessions with microstimulation in vPul (red) and dPul-p (blue) displayed on a T1-weighted MR image aligned to standard AC-PC space. (C) Coronal sections showing statistical $t$ maps of BOLD activation at the stimulation sites during unilateral low-current stimulation of dPul-p (blue) and vPul (red/yellow) in the right hemisphere combined across all three cognitive tasks. Schematic outlines were adapted from the NeuroMaps atlas (Rohlfing et al., 2012). dPul-p: posterior dorsal pulvinar, R: right, vPul: ventral pulvinar, Y: distance from AC-PC origin in the anterior/posterior plane in millimeters.

the dPul stimulation sites corresponded mostly to PM and the dorsal part of PL. The brachium of the SC (bsc) and other neighboring structures such as the reticular thalamic nucleus and the tail of the caudate nucleus were avoided. Moreover, in both animals anterior dPul stimulation sites (dPul-a) were indeed located more anterior in the anterior/posterior plane than posterior dPul sites (dPul-p). The dPul-a and dPul-p sites were $\sim 1 \mathrm{~mm}$ and $\sim 0.5 \mathrm{~mm}$ apart from each other in monkey $\mathrm{C}$ and monkey $\mathrm{B}$, respectively. In monkey $\mathrm{C}$ we additionally stimulated one site in vPul for direct comparison with the dPul-p stimulation site using a lower current strength $(100 \mu \mathrm{A})$. Fig. 3.3A shows the electrode position in an example session of vPul stimulation in a T2-weighted MR image with the slice package aligned to the chamber vertical axis and reconstructed in a T1-weighted MR image. As can be seen in the probability 
maps of the estimated electrode tip positions across stimulation sessions in Fig. 3.3B the vPul stimulation site mostly corresponded to the ventral part of PL whereas the dPul-p stimulation site corresponded to PM and the dorsal part of PL. For localization of the LIP stimulation sites we used the segregation of LIP into a dorsal (LIPd) and a ventral (LIPv) zone (Saleem \& Logothetis, 2007; Shipp \& Zeki, 1995). Fig. 3.1 C, D, and F and Fig. 3.2 C, $\mathrm{D}$, and $\mathrm{F}$ show examples of measured and reconstructed electrode positions and probability maps of the estimated electrode tip positions across sessions with LIPd stimulation for each animal, respectively. LIPd stimulation sites corresponded to the dorsal zone of LIP. Again, in both animals anterior stimulation sites (LIPd-a) were indeed located more anterior in the anterior/posterior plane than posterior sites (LIPd-p). In monkey C LIPd-a and LIPd-p sites were $\sim 2 \mathrm{~mm}$ apart from each other. In monkey B the two LIPd sites were separated by $\sim 3$ $\mathrm{mm}$.

\subsubsection{Behavioral paradigm}

For training and scanning, monkeys sat in custom-made horizontal MR compatible primate chairs in a sphinx position with their heads rigidly attached to the respective chair with a

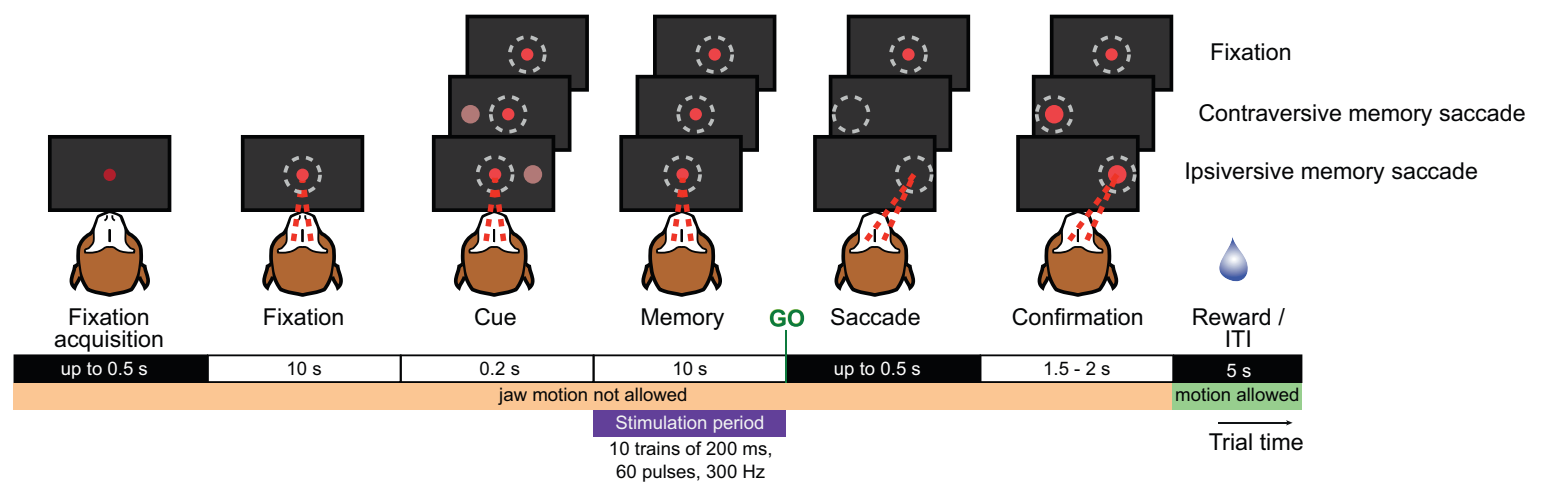

Fig. 3.4 Time course of one successful trial of the memory-saccade task and the fixation task, respectively. Gray dashed circles illustrate the animal's eye position but were not displayed on the screen. All trials started with a fixation period. Then, in memory-saccade trials a visual cue was presented in the contraversive (left) or ipsiversive (right) hemifield. The presentation of the cue was followed by a memory period in which pulvinar or LIP was electrically stimulated in half of the trials. The animals were to make a saccade towards the cued location as soon as the fixation spot disapppeared (GO) in order to see the upcoming target stimulus confirming correct saccade execution. In fixation trials the animals were to keep fixating the fixation spot until the end of the trial. In half of the trials stimulation was applied in the time window corresponding to the memory period in memory-saccade trials. Correct trials were rewarded with a fluid reward. Jaw motion was only allowed during reward delivery and the intertrial interval (ITI). 
PEEK headholder. In one third of the trials, the animals performed an eye fixation task. In the remaining two thirds of all trials, they performed memory-guided saccades. Fig. 3.4 shows a schematic of the behavioral tasks in experimental sessions. In training sessions the tasks were very similar except for a more variable timing to avoid that the animals would learn to predict the occurrence of trial events. Trials were initiated by fixating the central fixation spot (red dot, $0.25^{\circ}$ diameter). Memory-guided saccade trials continued with an eye fixation period (monkey C: $10 \mathrm{~s}$ in experimental sessions, 12 - $14 \mathrm{~s}$ in training sessions; monkey B: $10 \mathrm{~s}$ in experimental sessions, 9.25 - $10.25 \mathrm{~s}$ in training sessions). Subsequently, a light red, filled circle ( $1^{\circ}$ diameter) representing a visuospatial cue was presented for 200 $\mathrm{ms}$ either in the contraversive (left, contralateral to the side of stimulation) or the ipsiversive (right, ipsilateral to the side of stimulation) hemifield while the animals were maintaining central eye fixation. The offset of the cue determined the beginning of the memory period (monkey C: $10 \mathrm{~s}$ in experimental sessions, $12-14 \mathrm{~s}$ in training sessions; monkey B: $10 \mathrm{~s}$ in experimental sessions and $9.5-10.5 \mathrm{~s}$ in training sessions) in which the animals were to keep the cued spatial location active in memory and plan a saccadic eye movement towards this location while maintaining central eye fixation. After this memory period, the fixation spot disappeared determining the time the animals were allowed to execute the saccadic eye movement towards the remembered location. If the animals performed a saccade towards the correct location within a radius of $5^{\circ}$ to $7^{\circ}$ around the center of the cued location within $500 \mathrm{~ms}$, a target stimulus (red, filled circle, $1^{\circ}$ diameter) appeared at the saccade location to signal to the animal that the saccade had been performed correctly. After fixating the saccade location for another few seconds (monkey $\mathrm{C}$ : $2 \mathrm{~s}$ in experimental sessions, 2 - $3 \mathrm{~s}$ in training sessions; monkey B: $1.5 \mathrm{~s}$ in experimental sessions, $1-1.5 \mathrm{~s}$ in training sessions) a fluid reward was delivered. In the fixation task, the animals were required to maintain central eye fixation at the fixation spot until the end of the trial (monkey C: $22.2 \mathrm{~s}$ in experimental sessions, 26 - $31 \mathrm{~s}$ in training sessions; monkey B: $21.7 \mathrm{~s}$ in experimental sessions, 19.95 $22.45 \mathrm{~s}$ in training sessions) in order to get a fluid reward. In training sessions the fluid reward was preceded by a feedback sound. In both tasks, blink allowance time was $0.3 \mathrm{~s}$. Trials with fixation breaks exceeding an allowance window of $4^{\circ}$ to $5^{\circ}$ radius around the fixation spot and trials with incorrect or too slow saccade execution were aborted and not rewarded. In addition, video-based motion-detection systems (Pelco) were used to train the animals to minimize their jaw movements during the trials and to track jaw movements during scanning. Jaw movements were only allowed in the intertrial interval $(2 \mathrm{~s}$ after aborted trials with no reward, $5 \mathrm{~s}$ after correct trials with reward). Trials compromised by jaw motion were aborted. In half of the trials, we electrically stimulated either dPul, LIP, or vPul throughout the entire memory period in the memory-guided saccade task or in the corresponding time window 
in the fixation task starting at the time of the offset of the visual cue until the go signal (see section 3.2.3 for stimulation parameters). Table 3.1 gives an overview of the number of sessions, EPI runs, and successful trials per stimulation site, animal, and session for high-current stimulation in dPul and LIP. Table 3.2 gives a similar overview for low-current stimulation in vPul and dPul-p in monkey $\mathrm{C}$.

Visual stimuli were projected onto a custom-made MR compatible screen (800x600 pixels). Visual cues and targets were displayed at one of three locations per hemifield (six locations in total) with an eccentricity of $12^{\circ}$ of visual angle. Stimulus locations were arranged concentrically around the fixation spot at $0^{\circ}$ (mid left), $30^{\circ}$ (up left), $150^{\circ}$ (up right), $180^{\circ}$ (mid right), $210^{\circ}$ (down right), and $330^{\circ}$ (down left). Trials were presented in a pseudorandomized order to ensure a similar distribution of trial types throughout the duration of each fMRI run (see section 3.2.4 for functional data acquisition). Eye position was monitored at 60 $\mathrm{Hz}$ with an MR compatible infrared camera (Resonance Technology/Arrington Research) and was recorded simultaneously with stimulus and timing parameters and digital triggers from the scanner. Stimulus presentation, all behavioral control functions, and synchronization of the behavioral task with scanning were programmed in MATLAB (R2014a, 64-bit; The MathWorks, Inc., USA) and the Psychophysics Toolbox (Brainard, 1997).

\subsubsection{Data analysis}

\section{Behavioral analysis}

For behavioral analysis, all trials of all experimental sessions were pooled for each stimulation site separately. In order to test the effects of microstimulation on task performance, first, the overall hit rate for all control trials without stimulation and for all stimulation trials was calculated, respectively, and a Chi square test was performed to determine the effect of microstimulation on the frequency of successful trials. If this Chi square test revealed a significant difference between the frequency of successful trials in control and stimulation conditions, additional Chi square tests were performed for each task (fixation, contraversive memory saccade, ipsiversive memory saccade) separately to test whether microstimulation affected task performance in a task-specific manner. Since trials in control and stimulation conditions were only different starting from stimulation onset, similar analyses were performed on the frequency of trials aborted during and after the stimulation period, respectively, in order to detect changes in task performance that were time-locked to the delivery of current pulse trains and to assess the effect of stimulation on subsequent saccade execution.

All eye movements with a minimum velocity of $15 \%$ and a minimum duration of $10 \mathrm{~ms}$ were included in the analysis, including small saccades and eye blinks. The point in time 
Table 3.1 Number of EPI runs and successful trials per stimulation site, monkey, and session for high-current stimulation in anterior dorsal pulvinar (dPul-a), posterior dorsal pulvinar (dPul-p), anterior dorsal lateral intraparietal area (LIPd-a), and posterior dorsal lateral intraparietal area (LIPd-p).

\begin{tabular}{|c|c|c|c|c|c|}
\hline \multirow{2}{*}{$\begin{array}{l}\text { Stimulation } \\
\text { site }\end{array}$} & \multirow{2}{*}{$\begin{array}{l}\text { Session } \\
\text { number }\end{array}$} & \multicolumn{2}{|r|}{ Monkey C } & \multicolumn{2}{|r|}{ Monkey B } \\
\hline & & Runs & Successful trials & Runs & Successful trials \\
\hline \multirow{9}{*}{ dPul-a } & 1 & 3 & 69 & 7 & 133 \\
\hline & 2 & 7 & 165 & 8 & 126 \\
\hline & 3 & 6 & 132 & 7 & 124 \\
\hline & 4 & 8 & 158 & 7 & 112 \\
\hline & 5 & 7 & 140 & 7 & 124 \\
\hline & 6 & - & - & 8 & 147 \\
\hline & 7 & - & - & 6 & 106 \\
\hline & 8 & - & - & 6 & 120 \\
\hline & 9 & - & - & 7 & 136 \\
\hline \multirow{9}{*}{ dPul-p } & 1 & 3 & 62 & 7 & 142 \\
\hline & 2 & 6 & 120 & 8 & 165 \\
\hline & 3 & 6 & 137 & 7 & 132 \\
\hline & 4 & 6 & 143 & 8 & 167 \\
\hline & 5 & 5 & 90 & 6 & 128 \\
\hline & 6 & 6 & 107 & 7 & 133 \\
\hline & 7 & 9 & 163 & 9 & 168 \\
\hline & 8 & - & - & 6 & 114 \\
\hline & 9 & - & - & 8 & 120 \\
\hline \multirow{11}{*}{ LIPd-a } & 1 & 8 & 195 & 6 & 112 \\
\hline & 2 & 6 & 157 & 8 & 129 \\
\hline & 3 & 6 & 162 & 8 & 155 \\
\hline & 4 & 8 & 211 & 11 & 216 \\
\hline & 5 & 6 & 154 & 3 & 24 \\
\hline & 6 & - & - & 10 & 181 \\
\hline & 7 & - & - & 8 & 143 \\
\hline & 8 & - & - & 9 & 131 \\
\hline & 9 & - & - & 7 & 137 \\
\hline & 10 & - & - & 7 & 128 \\
\hline & 11 & - & - & 8 & 153 \\
\hline \multirow{10}{*}{ LIPd-p } & 1 & 6 & 157 & 6 & 123 \\
\hline & 2 & 8 & 199 & 8 & 156 \\
\hline & 3 & 5 & 131 & 7 & 122 \\
\hline & 4 & 9 & 246 & 8 & 138 \\
\hline & 5 & 6 & 155 & 7 & 128 \\
\hline & 6 & - & - & 6 & 121 \\
\hline & 7 & - & - & 6 & 123 \\
\hline & 8 & - & - & 6 & 111 \\
\hline & 9 & - & - & 6 & 123 \\
\hline & 10 & - & - & 7 & 139 \\
\hline
\end{tabular}


Table 3.2 Number of EPI runs and successful trials per stimulation site and session for low-current microstimulation in posterior dorsal pulvinar (dPul-p) and ventral pulvinar (vPul) in monkey C.

\begin{tabular}{lccc}
\hline Stimulation site & Session number & Runs & Successful trials \\
\hline & 1 & 7 & 129 \\
& 2 & 6 & 102 \\
dPul-p & 3 & 6 & 138 \\
& 4 & 5 & 108 \\
& 5 & 6 & 134 \\
& 6 & 6 & 150 \\
& 7 & 10 & 240 \\
\hline \multirow{4}{*}{ vPul } & 1 & 3 & 52 \\
& 2 & 9 & 239 \\
& 3 & 5 & 117 \\
& 4 & 8 & 187 \\
& 5 & 6 & 137 \\
& 6 & 7 & 174 \\
& 7 & 6 & 147 \\
\hline
\end{tabular}

when eye velocity passed the minimum velocity criterion determined eye movement onset. Movement offset was defined as the point in time when eye velocity dropped below $10 \%$ s. Using these detection criteria, the number of eye movements during the stimulation period was extracted for each trial and averaged across all trials of each experimental condition (control and stimulation for fixation, ipsiversive memory saccade, and contraversive memory saccade, respectively).

In order to test whether stimulation evoked eye movements, the number of eye movements during the stimulation period was then tested in a two-way ANOVA design with factors task (fixation, ipsiversive memory saccade, contraversive memory saccade) and stimulation (control, stimulation). Further post-hoc t tests were performed if the ANOVA revealed significant effects of stimulation to test if effects of stimulation on the number of eye movements were task-specific. To further investigate the effects of microstimulation on saccade execution, saccade latencies were extracted from all saccade trials and again tested in a two-way ANOVA design with factors task (ipsiversive memory saccade, contraversive memory saccade) and stimulation (control, stimulation). Significant effects of stimulation on saccade latencies were further tested using post-hoc $t$ tests.

\section{Estimation of electrode tip positions}

For assessment of the variability in electrode tip positions across sessions, for each experimental session the location of the electrode tip was estimated based on the respective 
T2-weighted image acquired with the slice package aligned to the chamber vertical axis (see section 3.2.4) using BrainVoyager (Version 2.4.2.2070, 64-bit; Brain Innovation BV). For each stimulation site, a sphere (radius: $0.5 \mathrm{~mm}$ ) was created around each session's estimated electrode tip position and a probability map was created based on the resulting volumes of interest showing the probability of overlapping voxels across all spherical volumes of interest. For better comparability, the resulting probability maps were then transformed into AC-PC space and overlaid onto the high-resolution, full-head T1-weighted anatomical image of each monkey.

\section{Functional data processing}

The first four EPI volumes were excluded from functional data analysis in order to eliminate transient effects of magnetic saturation. Preprocessing was performed using MATLAB (R2014a, 64-bit; The MathWorks, Inc., USA) and the NeuroElf toolbox (v1.0, http://neuroelf.net/). EPI data of each run was preprocessed using slice time correction and a high-pass temporal filter with a cut-off of three cycles per 15 min run. In addition, 3D motion correction was performed using the first functional volume included into the analysis as a reference. Coregistration and volume time course computation was done using BrainVoyager (Version 2.4.2.2070, 64-bit; Brain Innovation BV). First, the in-plane anatomical image of each session was coregistered to the high-resolution full-head T1-weighted anatomical scan in the AC-PC plane. Then, EPI runs were coregistered to the respective ACPC registered in-plane anatomical image using rigid body transformations with automated initial alignment followed by careful manual fine-tuning of the resulting alignment based on anatomical landmarks. Finally, volume time courses were computed in AC-PC space using $1 \mathrm{~mm} \times 1 \mathrm{~mm} \times 1 \mathrm{~mm}$ voxel size and intersecting a mask only including voxels within the brain with the volume time course data resulting in $\sim 89,000$ voxels in monkey $\mathrm{C}$ and $\sim$ 110,000 voxels in monkey B as the total number of voxels considered for FDR correction. Additionally, a $1.5 \mathrm{~mm}$ x $1.5 \mathrm{~mm}$ x $1.5 \mathrm{~mm}$ Gaussian kernel was applied for spatial smoothing of the volume time courses using MATLAB (R2014a, 64-bit; The MathWorks, Inc., USA) and the NeuroElf toolbox (v1.0, http://neuroelf.net/).

\section{GLM, ROI definition, and event-related averaging}

General linear models (GLMs) were computed in MATLAB (R2014a, 64-bit; The MathWorks, Inc., USA) using the NeuroElf toolbox (v1.0, http://neuroelf.net/). For successful trials, all trial events - cue/memory period contraversive/ipsiversive with and without stimulation, contraversive/ipsiversive saccade, and the corresponding time windows in fixation trials 
- were extracted and used as predictors for the GLM. In addition, there was one predictor representing reward delivery and one predictor for aborted trials. Based on these predictors, design matrices were created for convolution with the hemodynamic response function (time to positive peak: $3 \mathrm{~s}$, time to negative peak: $10 \mathrm{~s}$ ) in order to compute GLMs. For each stimulation site, data from all sessions were combined and analyzed using a fixed-effects GLM. For visualization of statistical maps, surfaces of both hemispheres and animals were reconstructed in BrainVoyager (Version 2.4.2.2070, 64-bit; Brain Innovation BV).

For each animal and stimulation site, regions of interest (ROIs) were defined based on event-related statistical t maps contrasting all stimulation conditions with the corresponding control conditions independent of the cognitive task. These statistical maps were created in BrainVoyager (Version 2.4.2.2070, 64-bit; Brain Innovation BV) using an FDR-corrected threshold of $q<0.05$. Then, the maps were imported into MATLAB (R2014a, 64-bit; The MathWorks, Inc., USA) and the NeuroElf toolbox (v1.0, http://neuroelf.net/) where a clustertable including subclusters using a cluster threshold of $\mathrm{k} \geq 20$ was created. The resulting clusters were exported as regions of interest and then revised using BrainVoyager (Version 2.4.2.2070, 64-bit; Brain Innovation BV) and a rhesus monkey atlas (Saleem \& Logothetis, 2007). That is, clusters covering more than one brain region were split accordingly by extracting a maximum volume of $2.5 \mathrm{~mm} \times 2.5 \mathrm{~mm} \times 2.5 \mathrm{~mm}$ to $3.5 \mathrm{~mm} \times 3.5 \mathrm{~mm}$ x $3.5 \mathrm{~mm}$ around the center of activity belonging to a particular brain region avoiding spatial overlap between ROIs. Clusters that could not unambiguously be assigned to gray matter were excluded from the analysis.

For each stimulation site, BOLD time courses of each ROI were extracted using MATLAB (R2014a, 64-bit; The MathWorks, Inc., USA) and the NeuroElf toolbox (v1.0, http://neuroelf.net/). For event-related averaging, first, BOLD time course data was interpolated to increase temporal resolution to one data point per second. Then, for each condition time courses were computed relative to baseline, which was defined as the mean BOLD activity of the last $3 \mathrm{~s}$ of the initial fixation period before cue presentation in memorysaccade trials or the corresponding time window in fixation trials. Then, BOLD time courses were averaged across trials per condition.

\section{Analysis across ROIs}

For analysis across ROIs, the mean BOLD response between 2 and $9 \mathrm{~s}$ of the memory period or the corresponding time window in fixation trials was calculated for each ROI. ROIs with a mean BOLD response deviating more than 2.5 standard deviations from the mean BOLD response in control conditions across all ROIs relevant for the respective ROI analysis (see below) were excluded from further analysis. 
In order to investigate whether the magnitude of stimulation effect on BOLD responses depended on the cognitive task, we calculated the stimulation effect for each task and ROI as the difference between the mean BOLD response in the respective stimulation and control condition. Stimulation effects were then analyzed across ROIs using a two-way repeated measures ANOVA (rmANOVA) with factors task (ipsiversive memory saccade, contraversive memory saccade, fixation) and stimulation (control, stimulation) for each hemisphere separately. Significant effects were further investigated using paired-sample $t$ tests.

Moreover, we were interested in testing if microstimulation added activity to the initial BOLD response of an ROI in control conditions or whether stimulation rather leads to multiplication of the BOLD response in control conditions. To this end, we fitted the relationship between BOLD responses in control conditions and BOLD responses during stimulation with an additive model $(\mathrm{f}(\mathrm{x})=\mathrm{a}+\mathrm{x})$ and a multiplicative model $(\mathrm{f}(\mathrm{x})=\mathrm{a} \cdot \mathrm{x})$ for each task and hemisphere separately. We then evaluated the resulting adjusted R-squared values as indicators of goodness of fit.

For testing whether stimulation changed the spatial tuning of ROIs, for each ROI we assessed the extent of contraversive selectivity, i.e., whether the BOLD response of an ROI was stronger to visual cues/saccade preparation presented in/towards spatial locations in the hemifield contralateral to the side of stimulation (contraversive memory saccade conditions). To quantify contraversive selectivity, we calculated a contraversive selectivity index (CSI) by subtracting the mean BOLD response in ipsiversive trials from the mean BOLD response in contraversive trials for control and stimulation conditions for each ROI. Positive CSI values reflect contraversive tuning. Negative CSI values reflect ipsiversive tuning, i.e. tuning towards the side of space that is ipsilateral to the side of stimulation (ipsiversive memory saccade conditions). CSIs for control trials and stimulation trials were then compared across ROIs for each hemisphere separately using paired-sample $t$ tests.

Finally, in order to test whether stimulation effects on BOLD responses depended on the spatial tuning of ROIs, we categorized ROIs of both hemispheres according to their contraversive selectivity as indicated by CSI values for control conditions (positive vs. negative values) and tested the differences between the mean BOLD response in the respective stimulation and control condition of each task in a two-way mixed ANOVA with betweensubjects factor contraversive selectivity (contraversive tuning, ipsiversive tuning) and withinsubjects factor task (ipsiversive memory saccade, contraversive memory saccade, fixation). Interaction effects were further investigated using paired-sample $t$ tests to compare stimulation effects between tasks for ROIs with contraversive and ipsiversive tuning, separately. 

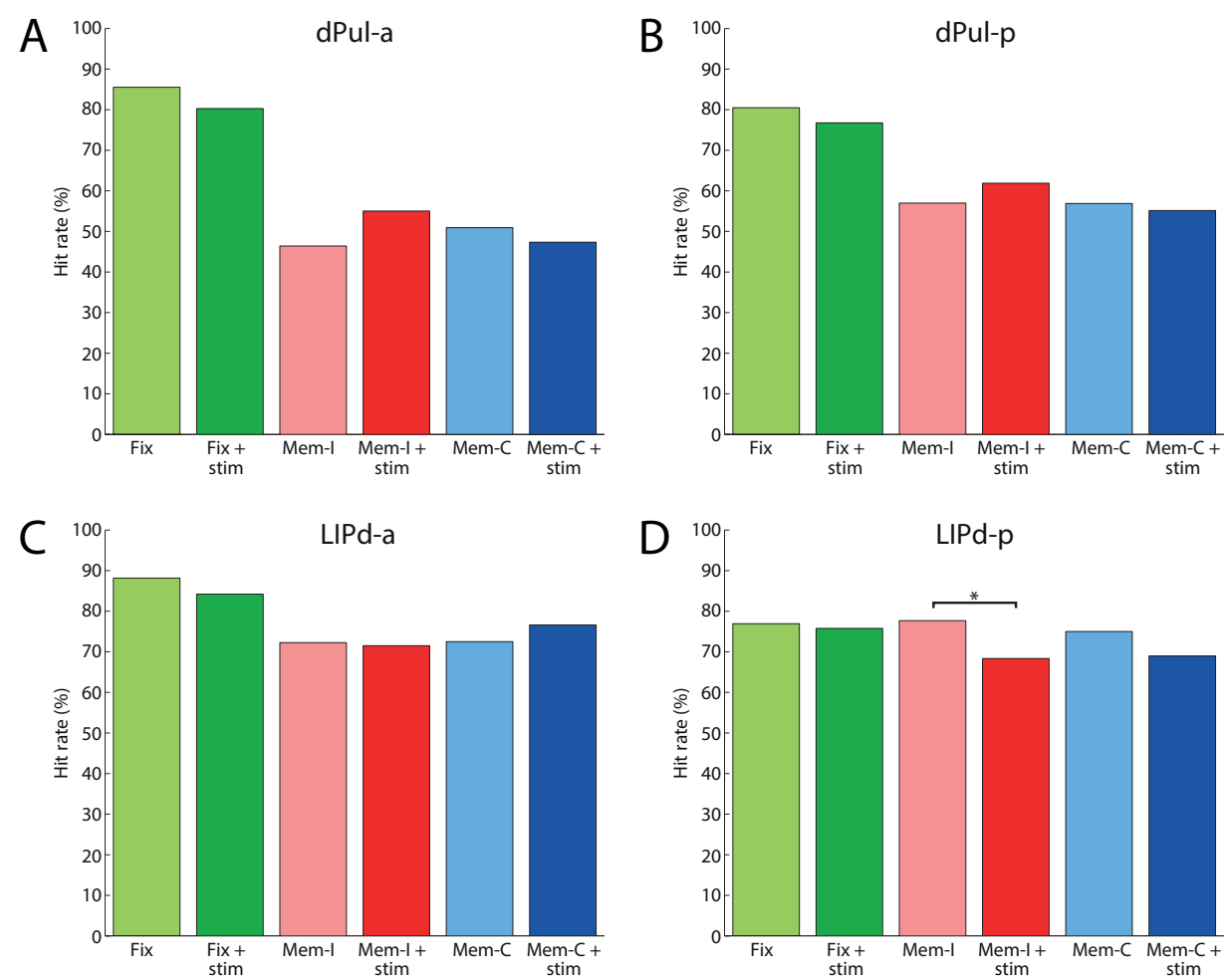

Fig. 3.5 Hit rates (proportion of successful trials) in the fixation, ipsiversive memory saccade, and contraversive memory saccade task in control (Fix, Mem-I, and Mem-C) and stimulation trials (Fix + stim, Mem-I + stim, Mem-C + stim) for stimulation in dPul-a (A), dPul-p (B), LIPd-a (C), and LIPd-p (D) in monkey C. dPul-a: anterior dorsal pulvinar, dPul-p: posterior dorsal pulvinar, LIPd-a: anterior dorsal lateral intraparietal area, LIPd-p: posterior dorsal lateral intraparietal area, $* \mathrm{p}<0.05$.

\subsection{Results}

\subsubsection{Task performance and eye movements}

In monkey $\mathrm{C}$, overall dPul-a stimulation neither affected hit rate nor the number of trials aborted in and after the stimulation period, respectively (all $\chi^{2} \mathrm{~s}(3) \leq 0.83$, all $p \mathrm{~s} \geq 0.3624$, see Fig. 3.5A, Fig. 3.7A, and Fig. 3.9A). Similar results were found for dPul-p stimulation (all $\chi^{2} \mathrm{~s}(3) \leq 1.31$, all $p \mathrm{~s} \geq 0.2523$, see Fig. 3.5B, Fig. 3.7B, and Fig. 3.9B). In monkey B, overall hit rate and the number of trials aborted in the stimulation period were not affected by dPul-a stimulation either (both $\chi^{2} \mathrm{~s}(3) \leq 2.494$, all $p \mathrm{~s} \geq 0.1143$, see Fig. 3.6A and Fig. 3.8A). However, dPul-a stimulation led to an overall increase in the number of trials aborted after the stimulation period $\left(\chi^{2}(3)=15.642, p<0.001\right)$ which was mainly driven by an impairment in making saccades to cued locations in the contraversive (left) hemifield, 

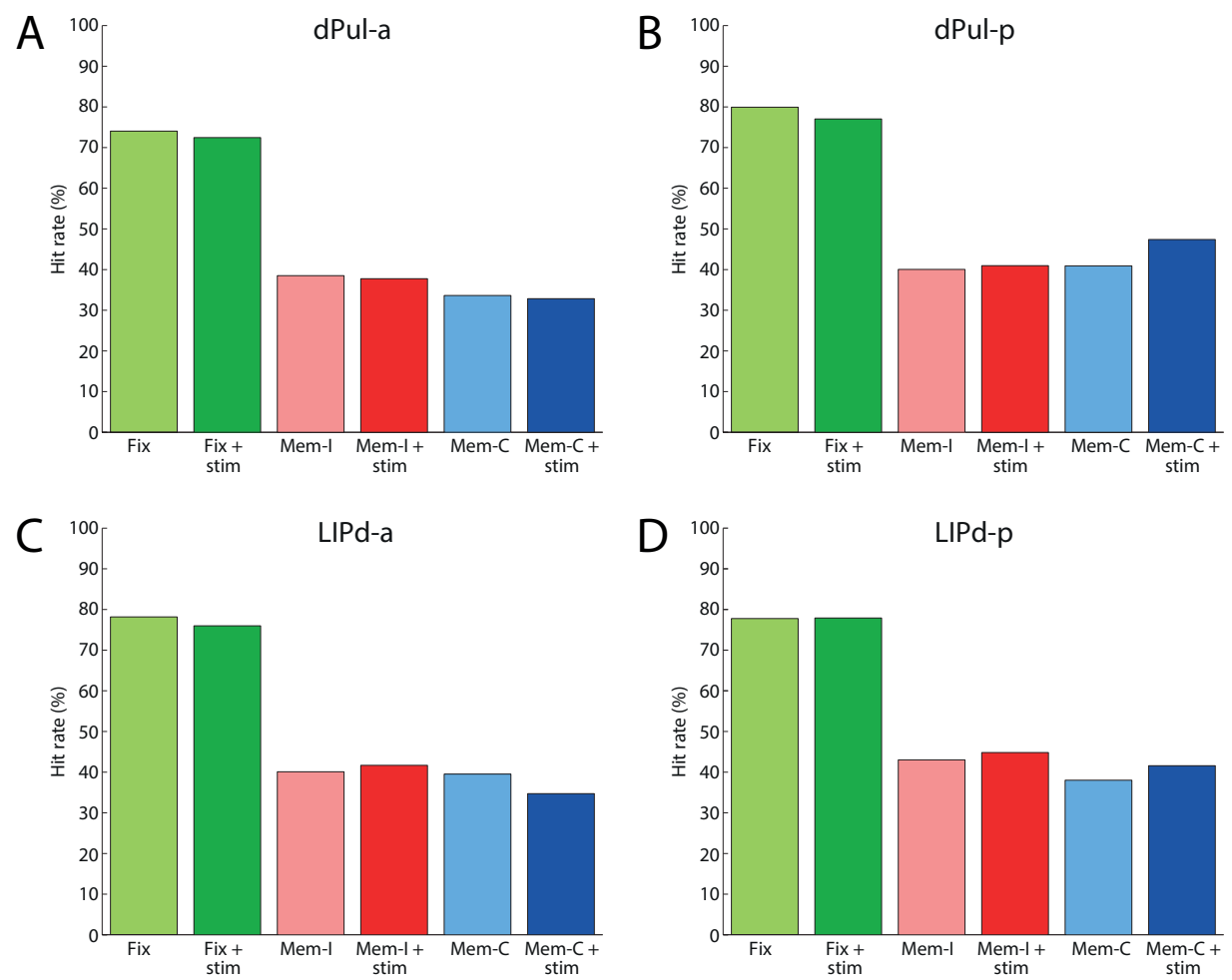

Fig. 3.6 Hit rates (proportion of successful trials) in monkey B. Same conventions as in Fig. 3.5.

i.e. the hemifield contralateral to the side of stimulation $\left(\chi^{2}(3)=29.03, p<0.001\right.$, see Fig. 3.10A). dPul-p stimulation, however, did not have an effect on the number of trials aborted after the stimulation period $\left(\chi^{2}(3)=1.48, p=0.2240\right.$, see Fig. 3.10B). Overall hit rate was not affected by dPul-p stimulation either $\left(\chi^{2}(3)=2.21, p=0.1370\right.$, see Fig. 3.6B) but there was a significant effect on the number of trials aborted in the stimulation period $\left(\chi^{2}(3)\right.$ $=3.67, p<0.05)$ with a significantly decreased number of aborted trials in the contraversive memory saccade task $\left(\chi^{2}(3)=5.37, p<0.05\right.$, see Fig. 3.8B).

LIPd-a stimulation did not have a significant effect on hit rate or the number of aborted trials (monkey $\mathrm{C}$ : all $\chi^{2} \mathrm{~s}(3) \leq 1.11$, all $p \mathrm{~s} \geq 0.2930$, see Fig. 3.5C, Fig. 3.7C, and Fig. 3.9C; monkey B: all $\chi^{2} \mathrm{~s}(3) \leq 3.63$, all $p \mathrm{~s} \geq 0.0568$, see Fig. 3.6C, Fig. 3.8C, and Fig. 3.10C). By contrast, LIPd-p stimulation led to a significantly lower overall hit rate in monkey $\mathrm{C}$ $\left(\chi^{2}(3)=5.03, p<0.05\right)$, which was mainly driven by a lower number of successful trials in the ipsiversive memory saccade task with stimulation compared to the control condition $\left(\chi^{2}(3)=4.48, p<0.05\right.$, see Fig. 3.5D). However, there was no significant increase in the number of trials aborted during or after the stimulation period $\left(\chi^{2}(3)=0.59, p=0.4416\right.$ and $\chi^{2}(3)=3.18, p=0.0747$, respectively) which might account for the decreased hit rate (see Fig. 3.7D, and Fig. 3.9D). In monkey B, LIPd-p stimulation did not affect overall hit rate 

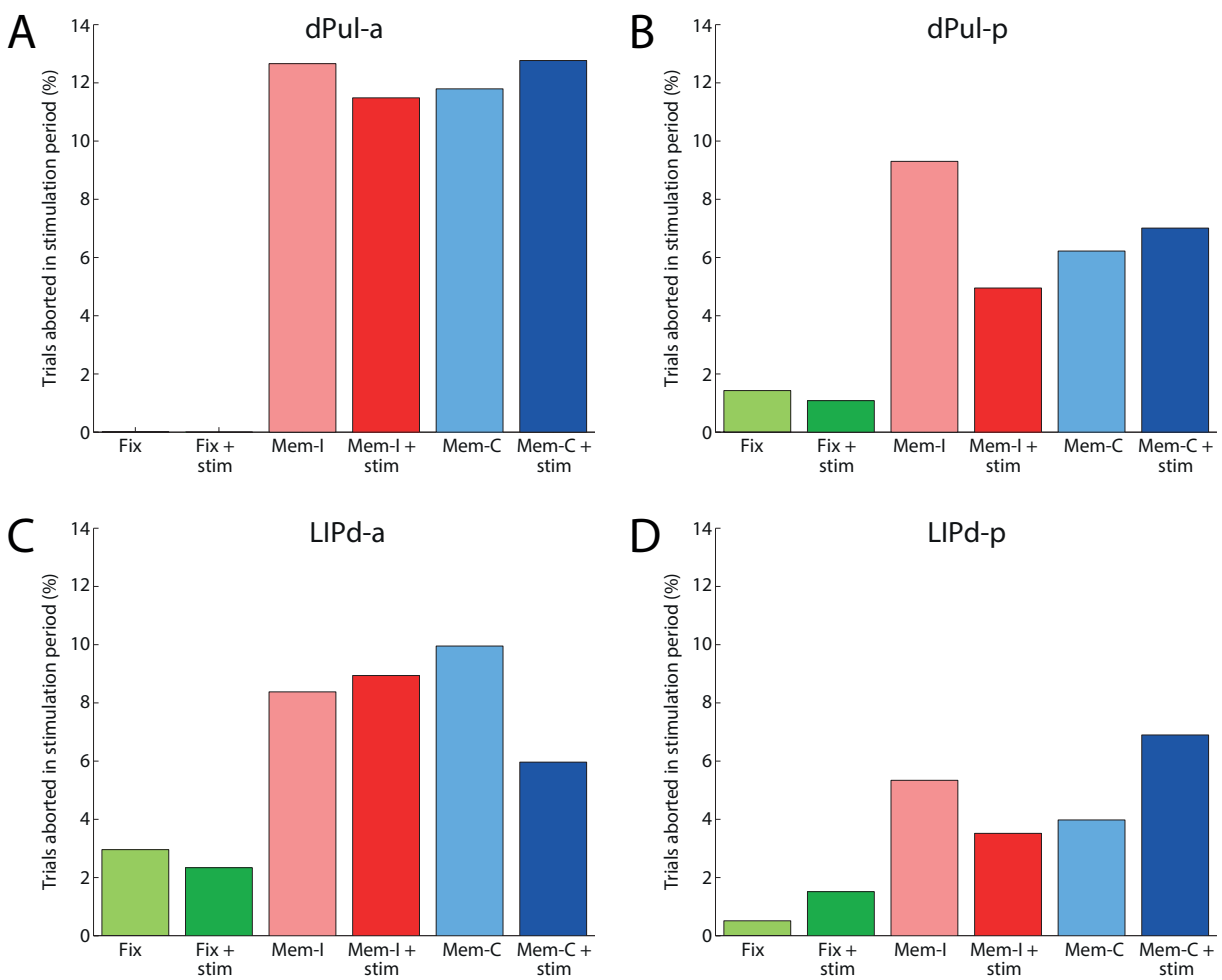

Fig. 3.7 Proportions of trials aborted in the stimulation period in the fixation, ipsiverive memory saccade, and contraversive memory saccade task in control (Fix, Mem-I, Mem-C) and stimulation trials (Fix + stim, Mem-I + stim, Mem-C + stim) for stimulation in dPul-a (A), dPul-p (B), LIPd-a (C), and LIPd-p (D) in monkey C. dPul-a: anterior dorsal pulvinar, dPul-p: posterior dorsal pulvinar, LIPd-a: anterior dorsal lateral intraparietal area, LIPd-p: posterior dorsal lateral intraparietal area, $* \mathrm{p}<$ 0.05 .

$\left(\chi^{2}(3)=1.84, p=0.1753\right.$, see Fig. 3.6D) or the number of trials aborted in the stimulation period $\left(\chi^{2}(3)=1.37, p=0.2424\right.$, see Fig. 3.8D) but led to a significant decrease in the overall number of trials aborted after the stimulation period $\left(\chi^{2}(3)=4.07, p<0.05\right)$. However, none of the comparisons between stimulation and control trials for each task separately reached significance (all $\chi^{2} \mathrm{~s}(3) \leq 2.70$, all $p \mathrm{~s} \geq 0.1005$, see Fig. 3.10D).

Even though dPul-a stimulation did not affect task performance in monkey $\mathrm{C}$, the two-way ANOVA on the number of eye movements in the stimulation period revealed a significant main effect of stimulation $(F(1,658)=127.06, p<0.001)$ with an increased number of eye movements in stimulation trials compared to the control condition in all three tasks (see Fig. 3.11A, $t(240)=6.22, t(195)=6.10$, and $t(223)=7.53$, respectively, all $p$ s $<$ 0.001). The two-way ANOVA on saccade latencies did not reveal significant effects of dPul-a stimulation (all $F \mathrm{~s}(1,418) \leq 2.30$, all $p \mathrm{~s} \geq 0.1305$, see Fig. 3.13A). In contrast, there 

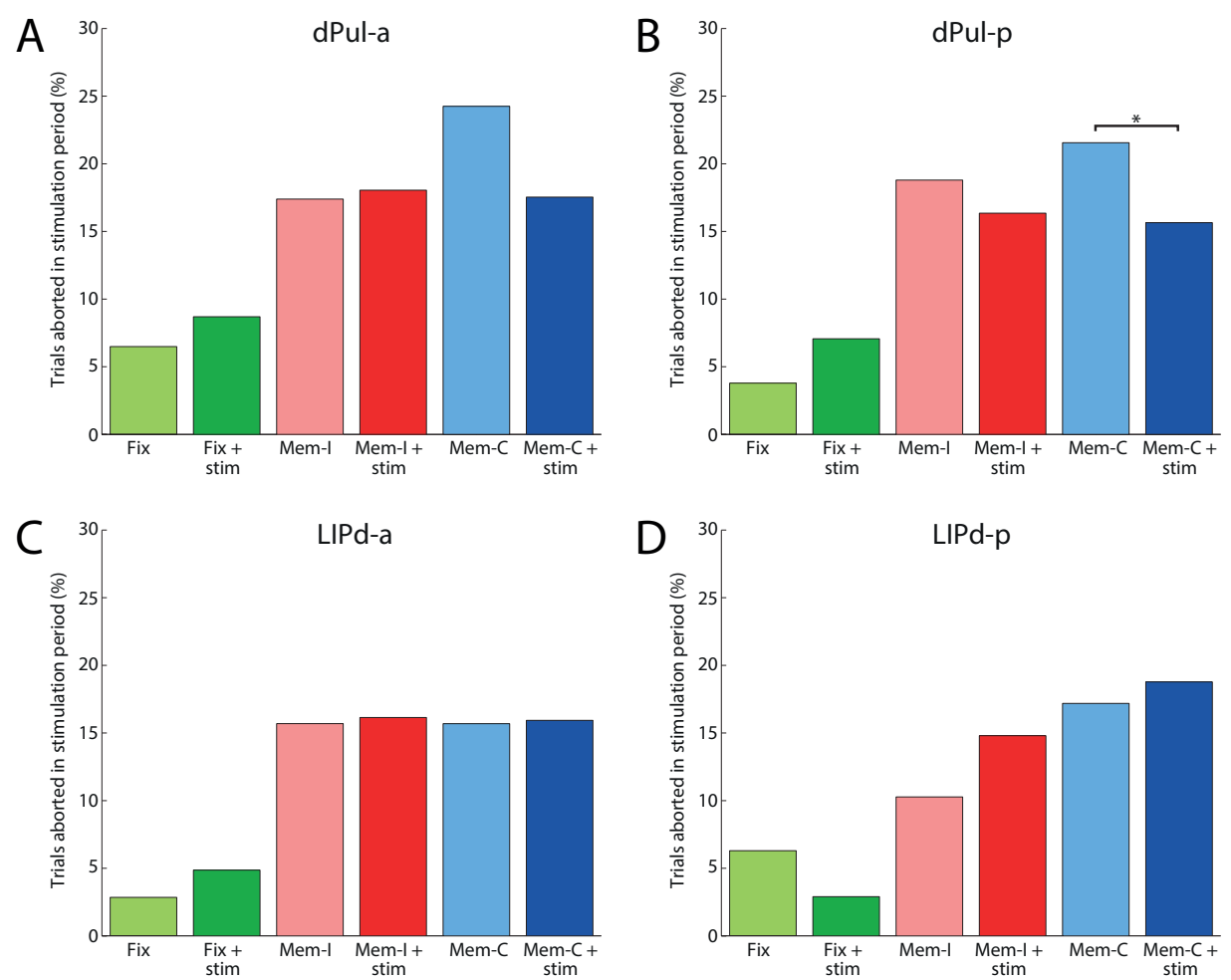

Fig. 3.8 Proportions of trials aborted in the stimulation period in monkey B. Same conventions as in Fig. 3.7.

were no significant effects of dPul-p stimulation on the number of eye movements in the stimulation period (see Fig. 3.11B, main effect stimulation: $F(1,823)=0.07, p=0.7881$, task $\times$ stimulation interaction: $F(2,823)=0.46, p=0.6324)$ but the two-way ANOVA on saccade latencies showed significant effects of dPul-p stimulation (both $F(1,514) \mathrm{s} \geq 8.04$, both $p$ s < 0.001) with significantly longer latencies for contraversive saccades following stimulation compared to the control condition $(t(244)=4.17, p<0.001$, see Fig. 3.13B). In addition to significant effects on task performance, in monkey B dPul-a stimulation also significantly affected the number of eye movements in the stimulation period (see Fig. 3.12A, main effect stimulation: $F(1,1122)=18.49$, task $\times$ stimulation interaction: $F(2,1122)=$ 9.50 , both $p$ s $<0.001$ ) but with a significantly lower number of eye movements in the fixation and the contraversive memory saccade task $(t(392)=2.80$ and $t(364)=5.09$, respectively, both $p \mathrm{~s}<0.001)$. Moreover, the two-way ANOVA on saccade latencies revealed a significant main effect of stimulation $(F(1,727)=15.70, p<0.001)$. Further post-hoc $\mathrm{t}$ tests showed that saccades to cued locations in both the ipsiversive and the contraversive hemifield were significantly delayed compared to the control conditions (see Fig. 3.14A, $t(366)=2.95$ and $t(361)=2.68$, respectively, both $p s<0.01)$. dPul-p stimulation also significantly influenced the number of eye movements in the stimulation period as shown by a significant task $x$ 

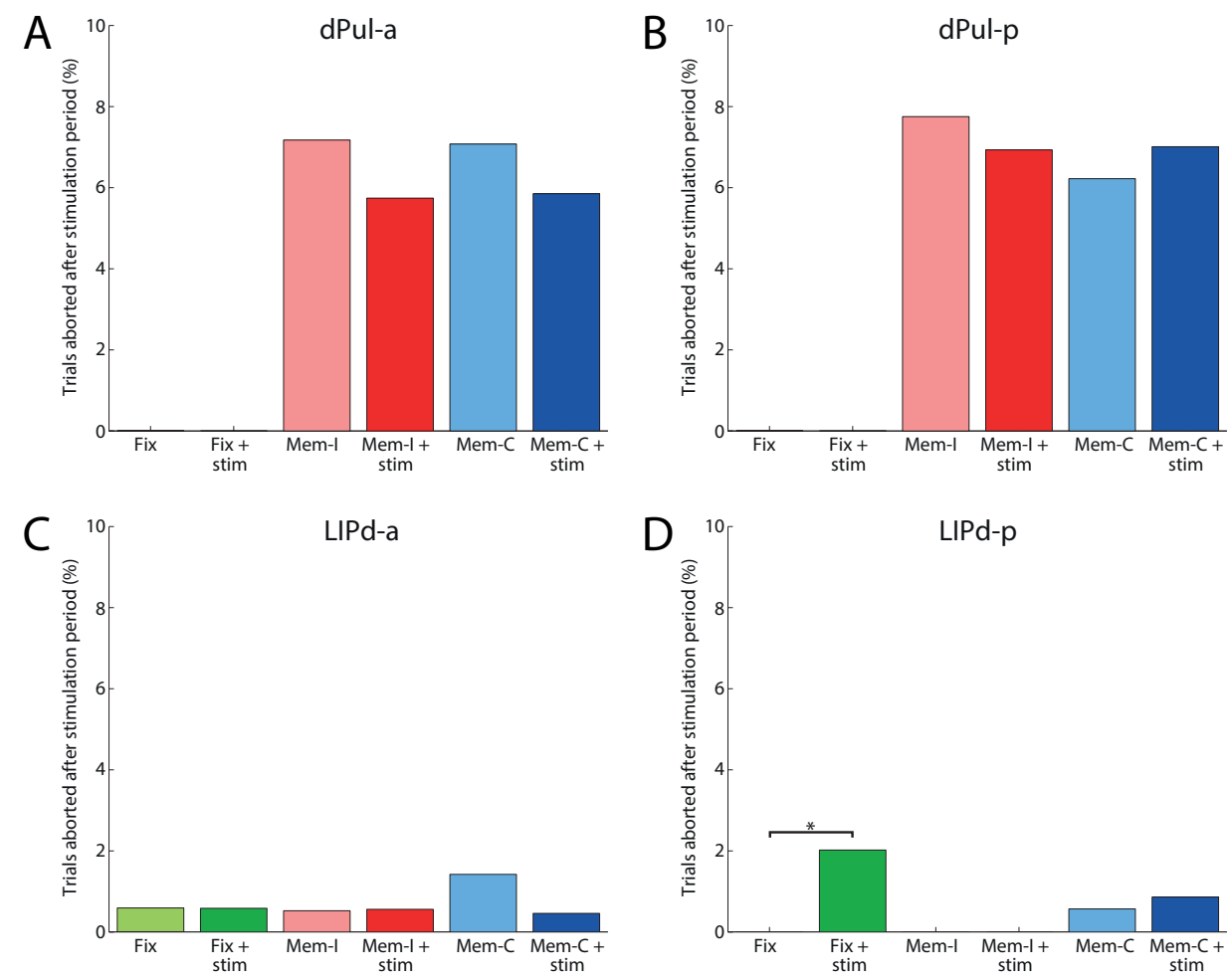

Fig. 3.9 Proportions of trials aborted after the stimulation period in the fixation, ipsiversive memory saccade, and contraversive memory saccade task in control (Fix, Mem-I, Mem-C) and stimulation trials (Fix + stim, Mem-I + stim, Mem-C + stim) for stimulation in dPul-a (A), dPul-p (B), LIPd-a (C), and LIPd-p (D) in monkey C. Note that in memory saccade tasks, the animal was to make a saccade towards the cued spatial location after the stimulation period. dPul-a: anterior dorsal pulvinar, dPul-p: posterior dorsal pulvinar, LIPd-a: anterior dorsal lateral intraparietal area, LIPd-p: posterior dorsal lateral intraparietal area, $* \mathrm{p}<0.05$.

stimulation interaction effect $(F(2,1263)=14.71, p<0.001)$. As can be seen in Fig. 3.12B, dPul-p stimulation led to a significantly higher number of eye movements in the ipsiversive memory saccade task $(t(424)=4.13, p<0.001)$ whereas the number of eye movements was decreased by dPul-p stimulation in the contraversive memory saccade task $(t(412)=$ $3.15, p<0.001)$. Similar to dPul-a stimulation, the two-way ANOVA on saccade latencies revealed a significant main effect of dPul-p stimulation with longer latencies of saccades to both the contraversive and the ipsiversive hemifield (see Fig. 3.14B, $F(1,833)=4.95, p<$ 0.05). However, for neither of the saccade tasks the difference in saccade latencies between stimulation and control trials reached significance in post-hoc $\mathrm{t}$ tests $(t(423)=1.60$ and $t(410)$ $=1.54$, both $p \mathrm{~s} \geq 0.1048$ ). 

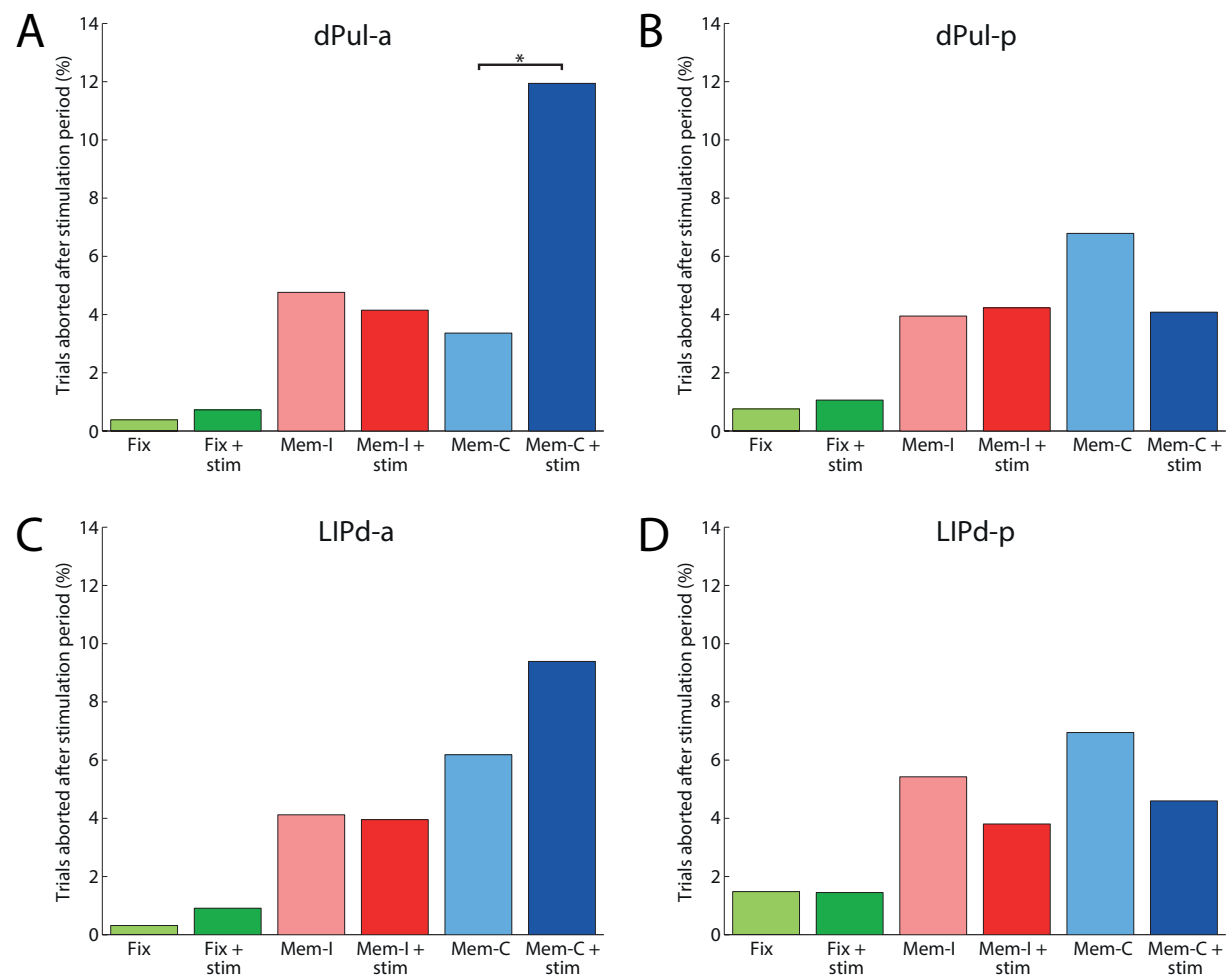

Fig. 3.10 Proportions of trials aborted after the stimulation period in monkey B. Same conventions as in Fig. 3.9.

Similar to dPul-a stimulation, the ANOVA on the number of eye movements in the stimulation period revealed a significant main effect of stimulation in LIPd-a in monkey $\mathrm{C}(F(1,873)=10.74, p<0.01)$. Further post-hoc $\mathrm{t}$ tests, however, showed that LIPd-a stimulation led to a significantly higher number of eye movements only in the contraversive memory saccade task (see Fig. 3.11C, $t(318)=3.09, p<0.01$ ). Saccade latencies, by contrast, were not affected by LIPd-a stimulation in monkey C (see Fig. 3.13C, main effect stimulation: $F(1,581)=1.05, p=0.3068$, task $\times$ stimulation interaction: $F(1,581)=0.10, p=0.7557)$. LIPd-p stimulation did not affect the number of eye movements in the stimulation period (see Fig. 3.11D, main effect stimulation: $F(1,882)=0.06, p=0.8063$, task $\times$ stimulation interaction: $F(2,882)=1.73, p=0.1773)$ but the two-way ANOVA on saccade latencies revealed a significant main effect of stimulation $(F(1,584)=10.42, p<0.01)$ mainly driven by an increased number of eye movements due to stimulation in the contraversive memory saccade task (see Fig. 3.13D, $t(290)=3.03, p<0.01$ ). In monkey B, LIPd-a stimulation also affected the number of eye movements in the stimulation period as shown by a significant main effect of stimulation $(F(1,1503)=22.89, p<0.001)$. In contrast to monkey C, LIPd-a stimulation in monkey B led to less eye movements (see Fig. 3.12C) with the difference between stimulation and control trials reaching significance in the fixation $(t(495)=3.24, p<$ 

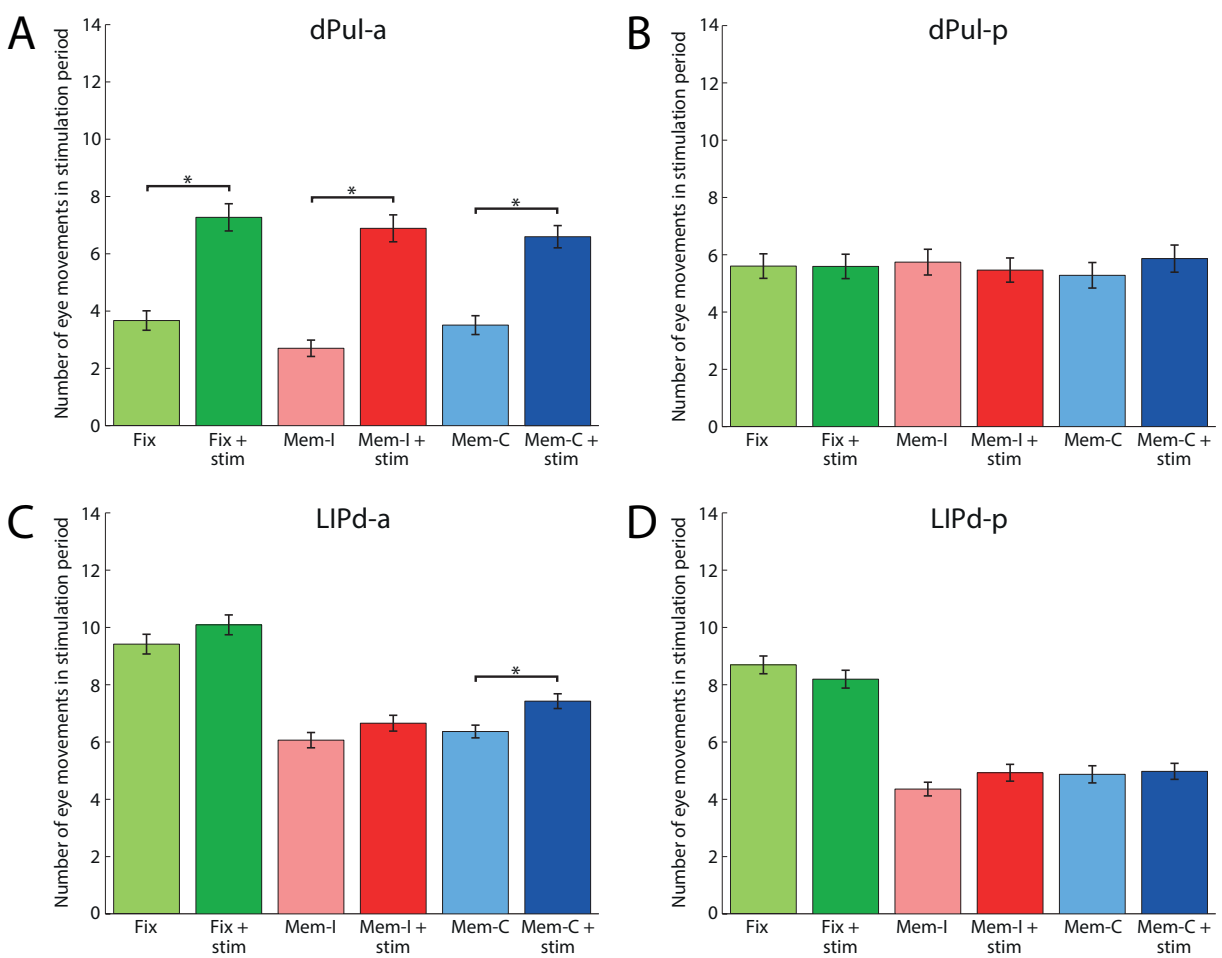

Fig. 3.11 Mean number of eye movements in the stimulation period and standard errors of means across trials in the fixation, ipsiversive memory saccade, and contraversive memory saccade task in control (Fix, MemI, Mem-C) and stimulation trials (Fix + stim, Mem-I + stim, Mem-C + stim) for stimulation in dPul-a (A), dPul-p (B), LIPd-a (C), and LIPd$\mathrm{p}(\mathrm{D})$ in monkey C. dPul-a: anterior dorsal pulvinar, dPul-p: posterior dorsal pulvinar, LIPd-a: anterior dorsal lateral intraparietal area, LIPd-p: posterior dorsal lateral intraparietal area, $* \mathrm{p}<0.05$.

$0.01)$ and the ipsiversive memory saccade task $(t(504)=3.24, p<0.01)$. Saccade latencies were also affected by LIPd-a stimulation in monkey B as shown by a significant main effect of stimulation $(F(1,1004)=11.11, p<0.001)$ and a significant task $\times$ stimulation interaction effect $(F(1,1004)=4.59, p<0.05)$. Post-hoc $\mathrm{t}$ tests showed that saccade latencies were significantly longer due to stimulation only in the contraversive memory saccade task (see Fig. 3.14C, $t(502)=3.66, p<0.001)$. In contrast to LIPd-a stimulation, stimulation in LIPd-p led to a higher number of eye movements in the stimulation period (see Fig. 3.12D) as shown by a significant main effect of stimulation $(F(1,1278)=41.94, p<0.001)$ and significant differences between stimulation and control trials for all three tasks (fixation: $t(423)=2.25, p$ $<0.05$; memory saccade right: $t(432)=4.69, p<0.001$; memory saccade left: $t(423)=4.26$, $p<0.001)$. However, LIPd-p stimulation did not have significant effects on saccade latencies 

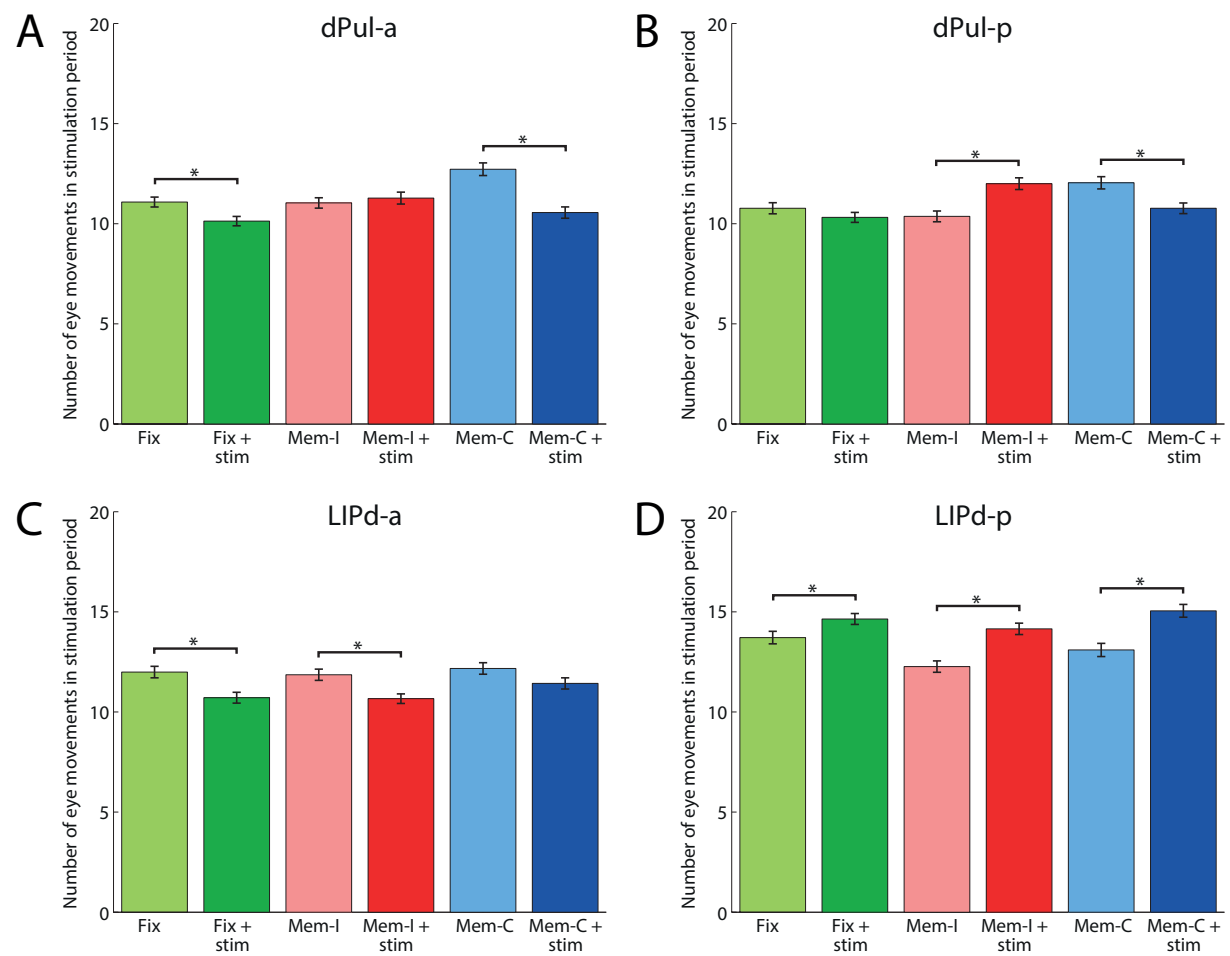

Fig. 3.12 Mean number of eye movements in the stimulation period and standard errors of means across trials in monkey B. Same conventions as in Fig. 3.11.

(see Fig. 3.14D, main effect stimulation: $F(1,854)=0.01, p=0.9190$, task $\times$ stimulation interaction: $F(1,854)=0.36, p=0.5493)$.

In summary, both dPul and LIP stimulation did not lead to major impairment in the fixation or the memory saccade task. Only LIPd-p stimulation was associated with a slightly lower hit rate for memory saccades to the ipsiversive hemifield in monkey C. However, this decrease in performance could not be explained by an increase in stimulation-related trial aborts. In monkey B, only dPul-a stimulation led to a significant impairment in the execution of memory saccades to the contraversive hemifield, i.e. the hemifield contralateral to the side of stimulation. dPul-a stimulation in monkey C and LIPd-p stimulation in monkey B led to a significant increase in the number of eye movements during the stimulation period in all three tasks suggesting that stimulation evoked eye movements. Evoked eye movements, however, did not lead to a higher number of trials aborted during the stimulation period. Both dPul and LIP stimulation led to significantly longer saccade latencies. More specifically, dPul-p and LIPd-p stimulation in monkey $\mathrm{C}$ were associated with longer latencies only for contraversive saccades. A similar effect was found for LIPd-a stimulation in monkey B. 
dPul-a stimulation in monkey B significantly increased latencies of saccades to both locations in the contraversive hemifield and locations in the ipsiversive hemifield.
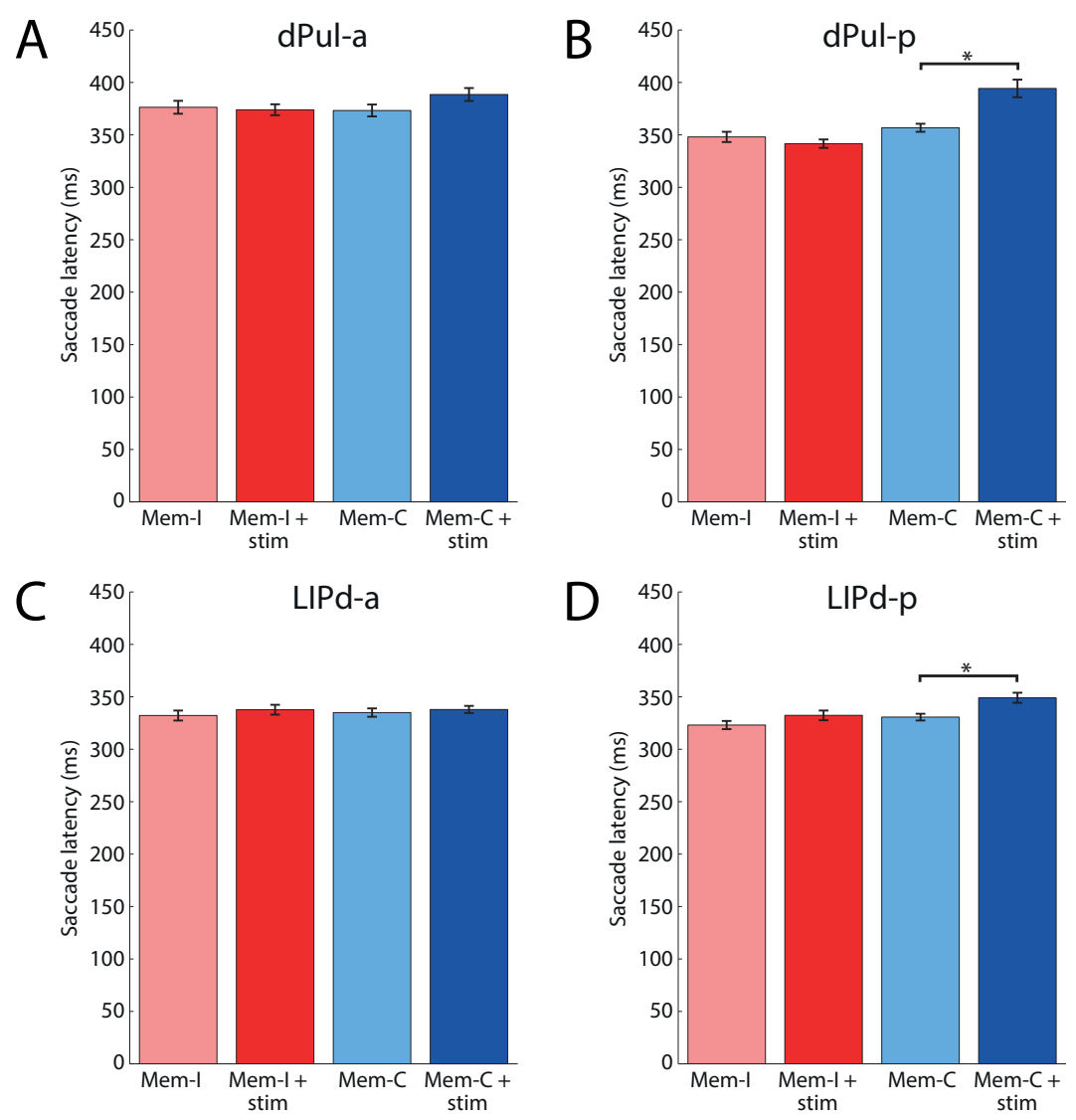

Fig. 3.13 Mean saccade latencies and standard errors of means across trials in the ipsiversive memory saccade and contraversive memory saccade task in control (Mem-I, Mem-C) and stimulation trials (Mem-I + stim, Mem-C + stim) for stimulation in dPul-a (A), dPul-p (B), LIPd-a (C), and LIPd-p (D) in monkey C. dPul-a: anterior dorsal pulvinar, dPul-p: posterior dorsal pulvinar, LIPd-a: anterior dorsal lateral intraparietal area, LIPd-p: posterior dorsal lateral intraparietal area, $* \mathrm{p}<0.05$. 

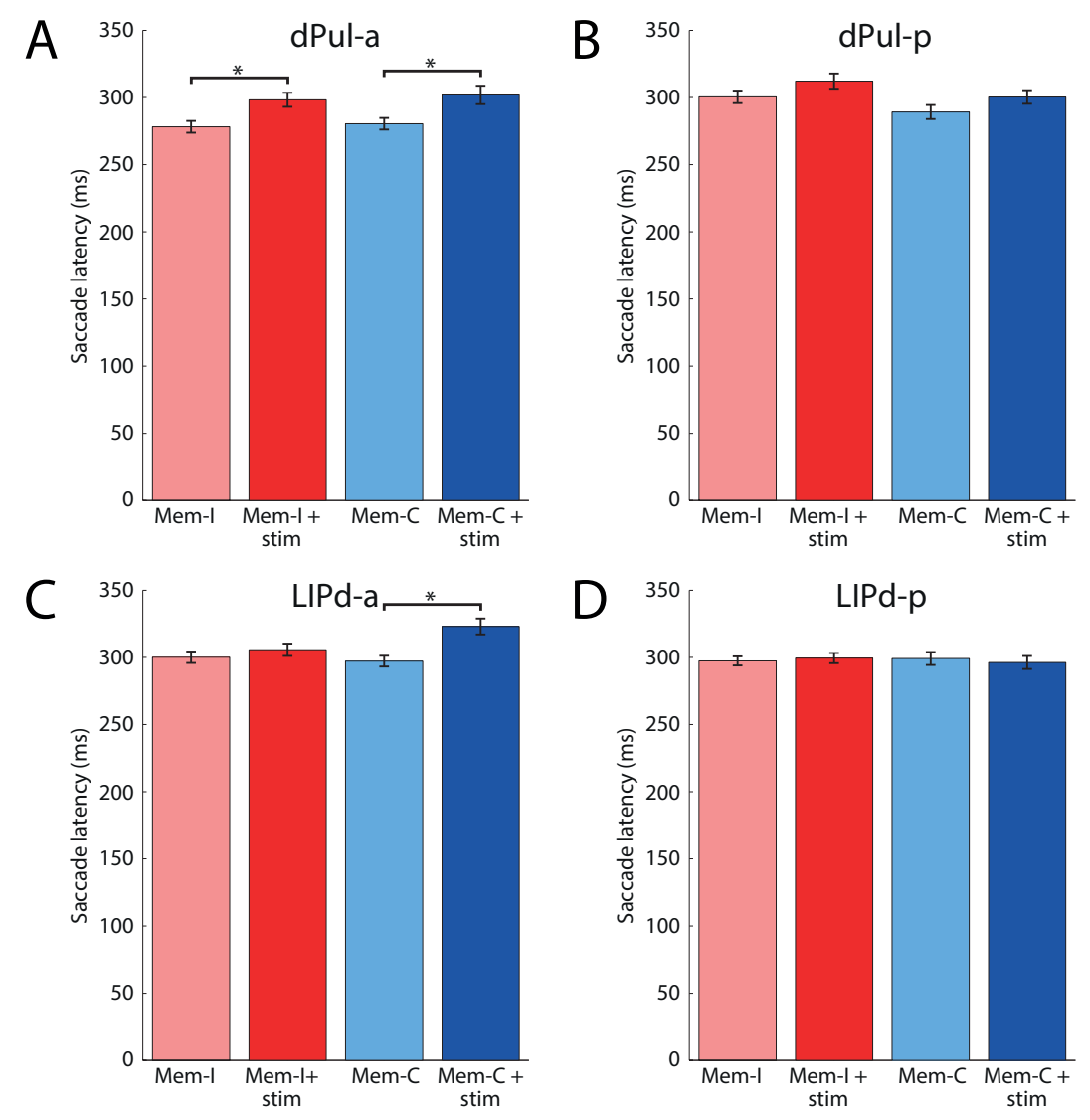

Fig. 3.14 Mean saccade latencies and standard errors of means across trials in monkey B. Same conventions as in Fig. 3.13. 

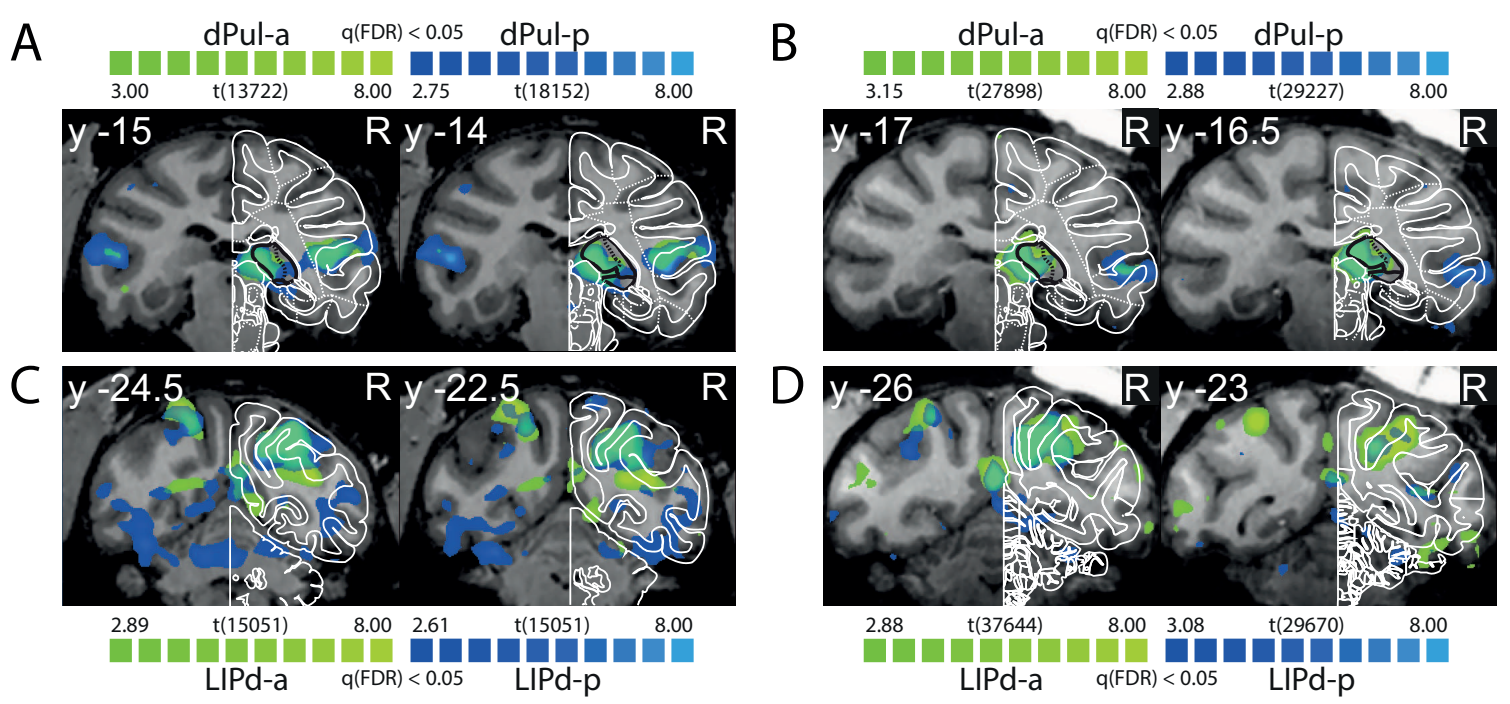

Fig. 3.15 Coronal sections showing statistical $t$ maps of BOLD activation at the stimulation sites during unilateral stimulation of $\mathrm{dPul}$ (A: monkey C, B: monkey B, green: dPul-a, blue: dPul-p) and LIP (C: monkey C, D: monkey B, green: LIPd-a, blue: LIPd-p) in the right hemisphere combined across all three cognitive tasks. Schematic outlines were adapted from the NeuroMaps atlas (Rohlfing et al., 2012). R: right, Y: distance from AC-PC origin in the anterior/posterior plane in millimeters.

\subsubsection{BOLD activity induced by dPul and LIP microstimulation}

As shown in Fig. 3.15A and B, in both animals high-current dPul stimulation elicited strong BOLD activity in dPul. dPul stimulation also induced activity in $\mathrm{SC}$ and, in monkey $\mathrm{C}$, in a small part of vPul. High-current stimulation of LIPd led to strong activation in the respective stimulated part of LIPd and in LIPv (see Fig. 3.15C and D). Note that BOLD activity in the close vicinity of the electrode cannot be measured due to signal dropouts caused by the use of metallic electrodes.

Fig. 3.16A shows statistical $\mathrm{t}$ maps reflecting BOLD activity induced by high-current dPul-a and dPul-p stimulation, respectively, combined across all three cognitive tasks in monkey $\mathrm{C}$ on the inflated brain surface based on the animal's individual anatomy. Fig. 3.18 shows the same $t$ maps on coronal sections. In the stimulated hemisphere, dPul stimulation led to significant BOLD activation in prefrontal cortex (a44, a45, a46) including FEF. dPul stimulation also activated premotor cortex (PMd/F2, PMv/F5) and led to strong activation in both the dorsal bank and the fundus of STS (IPa, FST, PGa, TPO, TAa, MST) as well as the ventral bank of STS (TEO, TEa, MT). Moreover, dPul stimulation induced BOLD activity in IPS (LIPd, LIPv, VIP, LOP, MIP), posterior cingulate cortex (PCC, see Fig. 3.18, y -23/-18/-10), insula, somatosensory cortex (areas 1,2, and 5, see Fig. 3.18, y -9/-23) and extrastriate (V2, V3, V4) and primary visual cortex (V1). Weak activation due to dPul 
stimulation could also be found in subcortical regions such as amygdala (see Fig. 3.18, y 1), caudate nucleus (see Fig. 3.18, y -3), other thalamic nuclei (see Fig. 3.18, y-11), and cerebellum (see Fig. 3.18, y -29). Although to a lesser extent, unilateral dPul stimulation also activated similar cortical brain regions in the opposite hemisphere in prefrontal cortex (a45, FEF), premotor cortex (PMd/F2, PMv/F5, see Fig. 3.18, y 0/1), STS (TEO, TPO, FST, MT, MST, 7a), IPS (AIP, LIPd, LIPv, LOP, PO), somatosensory cortex (areas 1 and 2, see Fig. 3.18, y -8), and visual cortex (V1, V2, V3, V4). Additional activation was found in the supplementary motor area (SMA, see Fig. 3.18, y -1). Subcortical activation could be found in putamen (see Fig. 3.18, y -1) and cerebellum (see Fig. 3.18, y -29). Fig. 3.17A and Fig. 3.19 show BOLD activity induced by dPul stimulation in monkey B on the inflated brain surface and on coronal sections, respectively. Similar to the activation pattern found in monkey C, dPul stimulation in monkey B activated cortical areas in prefrontal cortex (a45, a46, FEF), STS (TG, IPa, FST, TPO, TAa, TEO, TEa, TEm, MT, MST, 7a), IPS (LIPv, VIP, PIP, MIP), and somatosensory cortex (areas 1, 2, 3a/b, see Fig. 3.19, y -9.5/-0.5) as well as regions in extrastriate (V2, V3, V4) and primary visual cortex (V1, see also Fig. 3.19, y -37.5), insula, and PCC (see Fig. 3.19, y -23.5) in the stimulated hemisphere. Additionally, dPul stimulation in monkey B activated regions in orbital prefrontal cortex (areas 11, 12 , 13) and retrosplenial cortex (see Fig. 3.19, y -18.5). Again, activation could also be found in subcortical regions such as amygdala (see Fig. 3.19, y -2.5), pallidum (see Fig. 3.19, y -5.5), other thalamic nuclei (see Fig. 3.19, y -8.5/-12.5), cerebellum (see Fig. 3.19, y -31.5), and hippocampus (see Fig. 3.19, y -7.5). Unilateral dPul stimulation also induced BOLD activity in the opposite hemisphere in visual cortex (V1, V2, V3, V4) and STS (TEO) and, additionally, in orbital prefrontal cortex (area $12 \mathrm{~m}$ ) as well as in caudate nucleus (see Fig. 3.19, y 6.5).

In monkey $\mathrm{C}$ we also directly compared the BOLD activation patterns resulting from dPul-p and vPul stimulation. As mentioned above, high-current dPul-p stimulation activated a small part of vPul. In order to stimulate dPul-p more focally, we used a lower current strength $(100 \mu \mathrm{A})$ for the dPul-p-vPul comparison. As can be seen in the statistical t maps in Fig. 3.3C showing BOLD activity at the dPul-p and vPul stimulation site, respectively, low-current stimulation indeed led to activation that was locally more restricted to dPul-p than during high-current stimulation, which was spatially well-separated from stimulation-induced activity in vPul. Generally, low-current dPul-p stimulation elicited weaker BOLD responses in other brain areas than high-current stimulation (compare the blue maps in Fig. 3.16A and Fig. 3.16C). However, as can be seen in the statistical t map in Fig. 3.16C, low-current dPul-p stimulation activated similar areas in prefrontal cortex (a45, FEF), premotor cortex (PMv), STS (MT, FST, IPa), and extrastriate visual cortex (V3d) in the stimulated hemisphere as 

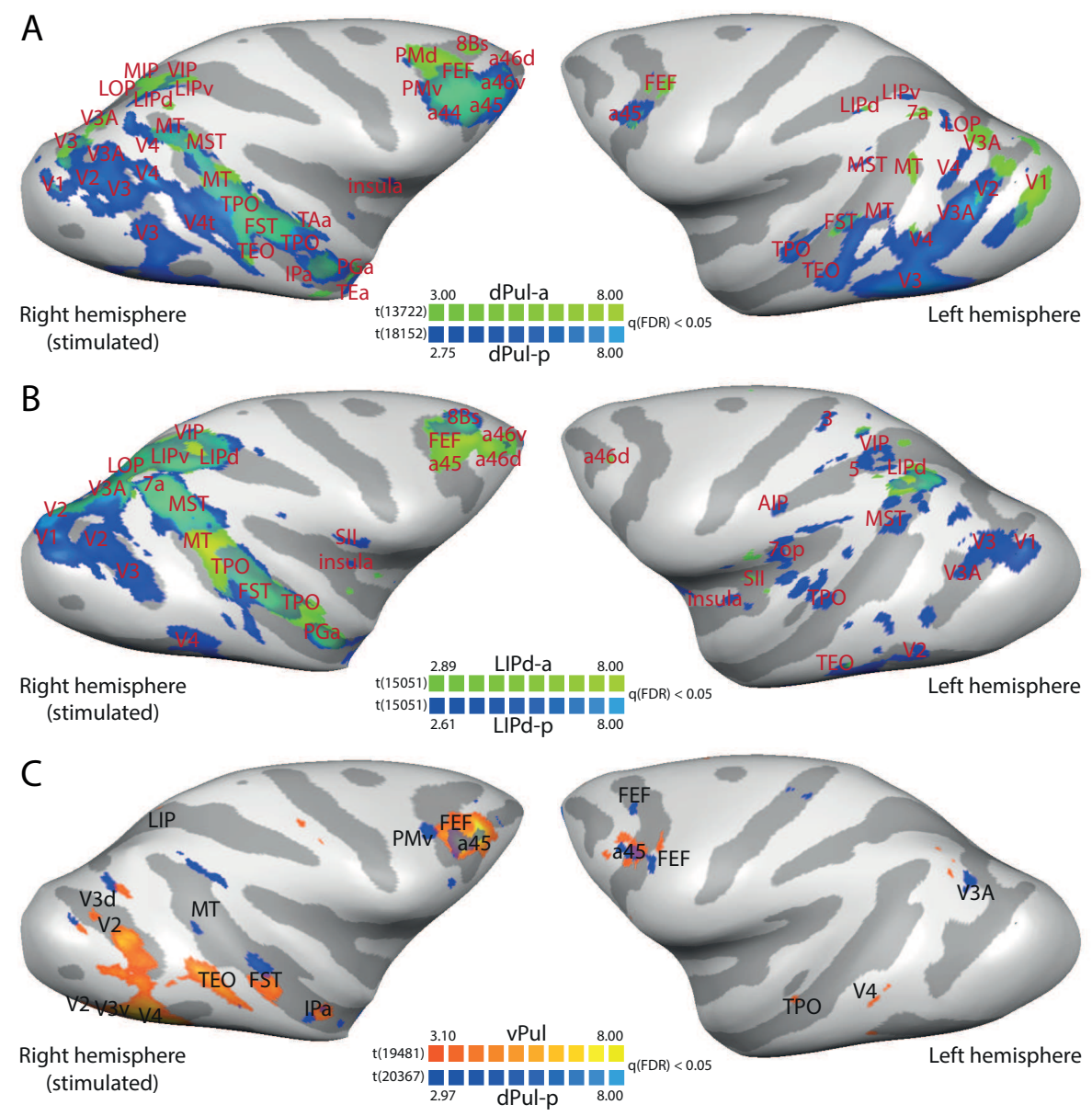

Fig. 3.16 Statistical t maps showing BOLD activation on the inflated brain surface of monkey $\mathrm{C}$ during unilateral high-current stimulation of dPul (A, green: dPul-a, blue: dPul-p) and LIP (B, green: LIPd-a, blue: LIPd-p) and during low-current stimulation (C) of dPul-p (blue) and vPul (red/yellow) in the right hemisphere combined across all three cognitive tasks.

high-current stimulation. Activation was also found in subcortical structures such as the caudate nucleus (see Fig. 3.20, y 0) and other parts of thalamus (see Fig. 3.20, y -7). As shown in Fig. 3.16C, similar to dPul-p stimulation, stimulation in vPul induced BOLD activity in STS (TEO, FST, IPa) and extrastriate visual cortex (V2, V3v, V4). Stimulation-induced activity in visual cortex was, however, more extensive than during dPul-p stimulation and also included primary visual cortex (V1, see Fig. 3.20, y -36). Moreover, vPul stimulation induced an increased BOLD response in IPS (LIPd/LIPv, see Fig. 3.20, y -25) and, interestingly, also strongly activated prefrontal cortex (a45, FEF) which might be caused by strong activation in SC (see Fig. 3.20, y -14/-13). Additional subcortical activity was found in amygdala (see Fig. 3.20, y -1). Again, both dPul-p and vPul stimulation elicited BOLD responses 

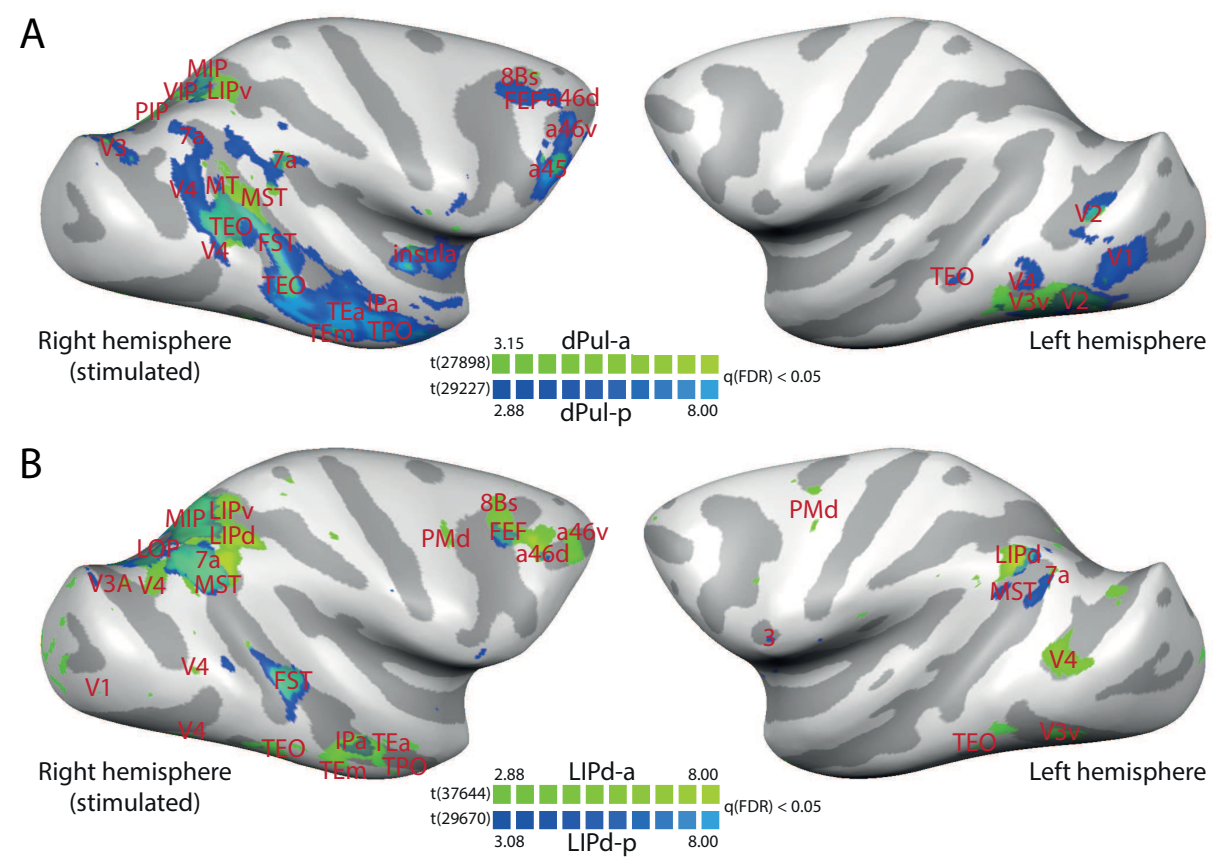

Fig. 3.17 Statistical $t$ maps showing BOLD activation on the inflated brain surface of monkey B during stimulation of dPul (A, green: dPul-a, blue: dPul-p) and LIP (B, green: LIPd-a, blue: LIPd-p) in the right hemisphere combined across all three cognitive tasks.

in the opposite hemisphere but to a lesser extent than in the stimulated hemisphere. More specifically, dPul-p stimulation-induced activation was found in prefrontal cortex (a45, FEF) and extrastriate visual cortex (V2, V3A). vPul stimulation also activated prefrontal cortex (a45, FEF) and extrastriate visual cortex (V4) in the opposite hemisphere and induced additional BOLD responses in STS (TPO, TEO, see also Fig. 3.20, y -18).

Fig. 3.16B shows statistical t maps overlaid onto the inflated brain surface reflecting BOLD activation induced by high-current LIPd-a and LIPd-p stimulation in monkey C, respectively. Fig. 3.21 shows the same statistical maps on coronal sections. Similar to dPul stimulation, LIPd stimulation activated cortical areas in the stimulated hemisphere in prefrontal cortex (a45, a46, area 8Bs, FEF) and STS (PGa, TPO, FST, MT, MST, 7a), other areas in IPS (LIPv, VIP, PIP, MIP, LOP, PO), somatosensory cortex (areas 1,2, and 5, SII, see Fig. 3.21, y -7/-14/-16/-22), PCC (see Fig. 3.21, y -14/-17/-24), insula, and primary (V1) and extrastriate visual cortex (V2, V3, V4). Interestingly, the activation patterns in prefrontal and visual cortex were different between LIPd-a and LIPd-p stimulation. LIPd-a stimulation caused strong BOLD activity in prefrontal cortex including FEF, a45, and a46d but only activated a few areas in visual cortex whereas prefrontal activation during LIPd-p stimulation was mainly restricted to area $8 \mathrm{Bs}$ and a46v but activation in visual cortex was quite extensive. 
LIPd stimulation, like dPul stimulation, also caused BOLD responses in subcortical regions including dPul and vPul (see Fig. 3.21, y -14/-15), caudate nucleus (see Fig. 3.21, y -14/-15), and amygdala (see Fig. 3.21, y -1). Unilateral LIPd stimulation also induced cortical BOLD activation in the opposite hemisphere in prefrontal cortex (a46), IPS (LIPd, LIPv, LOP, PO, VIP, AIP), STS (PGa, TPO, TEO, MST), insula, somatosensory cortex (areas 3a/b, 5, SII), PCC (see Fig. 3.21, y -17), and visual cortex (V1, V2, V3, V4). Additional activity was found in retrosplenial cortex (see Fig. 3.21, y -7) and primary motor cortex (see Fig. 3.21, y -5/-9). Moreover, LIPd stimulation activated subcortical regions in the opposite hemisphere such as dPul and vPul (see Fig. 3.21, y -15/-16), amygdala, and caudate (see Fig. 3.21, y -2). The BOLD activations found for LIPd stimulation in monkey B are shown in Fig. 3.17B and Fig. 3.22. Similar to the activation pattern resulting from LIPd stimulation in monkey C, LIPd stimulation in monkey B activated prefrontal cortex (a46, area 8Bs, FEF), STS (IPa, TEO, TE, TPO, FST, MT, MST, 7a), other areas in IPS (LIPv, VIP, MIP, LOP, PO), somatosensory cortex (area 5, see Fig. 3.22, y -29.5), PCC (see Fig. 3.22, y -21.5), and primary (V1) and extrastriate visual cortex (V2, V3, V4). Additional activation was found in premotor cortex $(\mathrm{PMd} / \mathrm{F} 2, \mathrm{PMv} / \mathrm{F} 4$, see Fig. 3.22, y 1.5) and orbital prefrontal cortex (area 111, see Fig. 3.22, y 21.5). Again, there were differences in the activation patterns caused by LIPd-a and LIPd-p stimulation. LIPd-a stimulation led to strong activation in prefrontal cortex and activated orbital prefrontal cortex and PMd. In contrast, LIPd-p stimulation-induced BOLD activity in frontal areas was generally less prominent and activation in premotor cortex was restricted to PMv. Subcortical activation induced by LIPd stimulation in monkey B was mainly restricted to cerebellum (see Fig. 3.22, y -31.5/-33.5) and hippocampus (see Fig. 3.22, y -10.5). In monkey B, LIPd stimulation also led to cortical BOLD activation in the opposite hemisphere in IPS (LIPd), STS (TEO, TEa, MST, 7a), PCC (see Fig. 3.22, y -20.5/-25.5), somatosensory cortex (area 3a/b, see Fig. 3.22, y -0.5), premotor cortex (PMd/F2, PMv/F5, see Fig. 3.22, y -2.5/1.5), orbital prefrontal cortex (area 12m, see Fig. 3.22, y 16.5), and visual cortex (V1, $\mathrm{V} 2, \mathrm{~V} 3, \mathrm{~V} 4)$. Additional cortical BOLD activation was found in anterior cingulate cortex (ACC, see Fig. 3.22, y -5.5). LIPd stimulation-induced activity in subcortical regions was found in hippocampus and hypothalamic nucleus (see Fig. 3.22, y -6.5). 


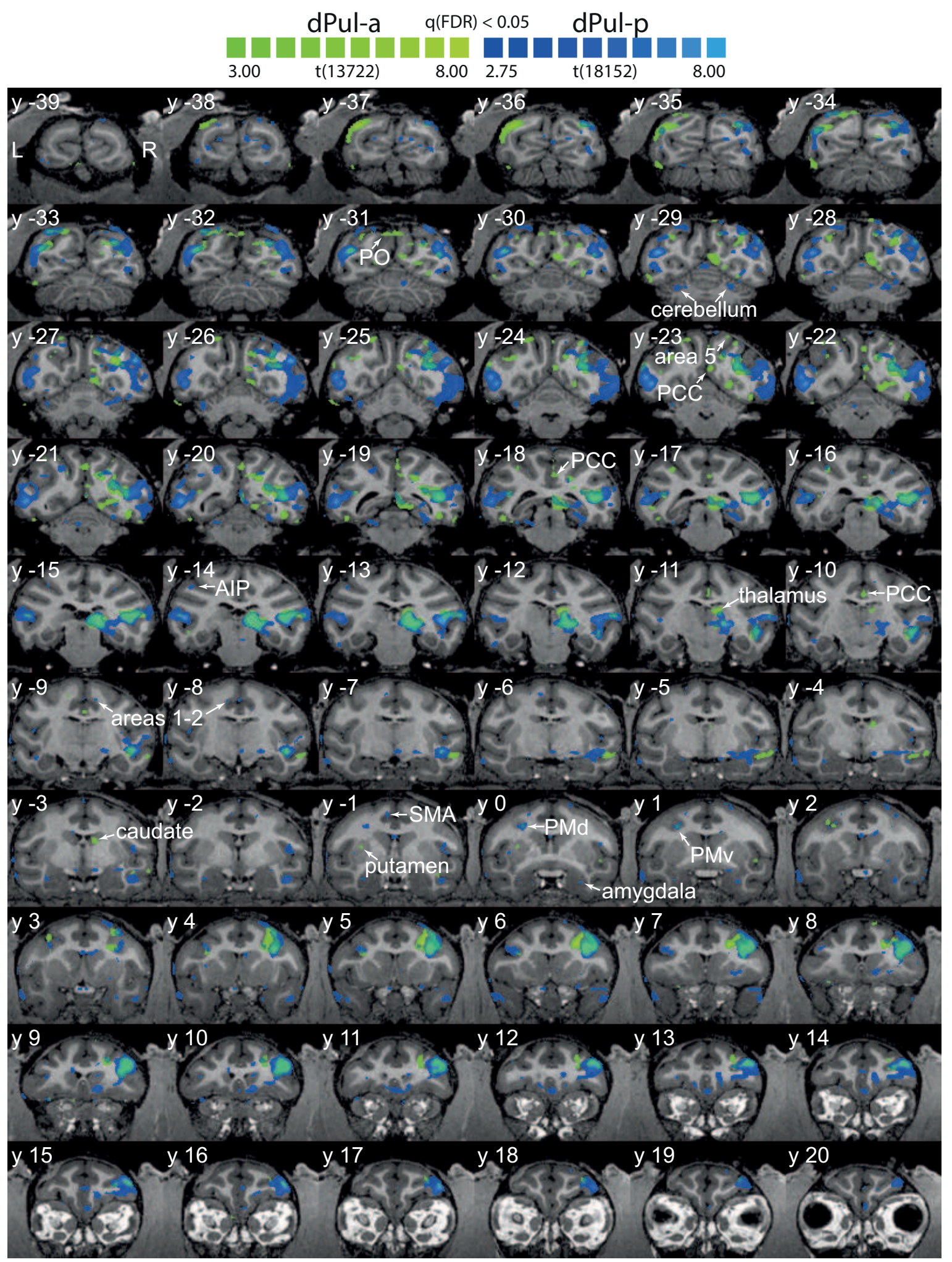

Fig. 3.18 Coronal sections showing statistical t maps of BOLD activation during unilateral high-current stimulation of dPul-a (green) and dPul-p (blue) in the right hemisphere combined across all three cognitive tasks in monkey C. L: left, R: right, Y: distance from AC-PC origin in the anterior/posterior plane in millimeters. 


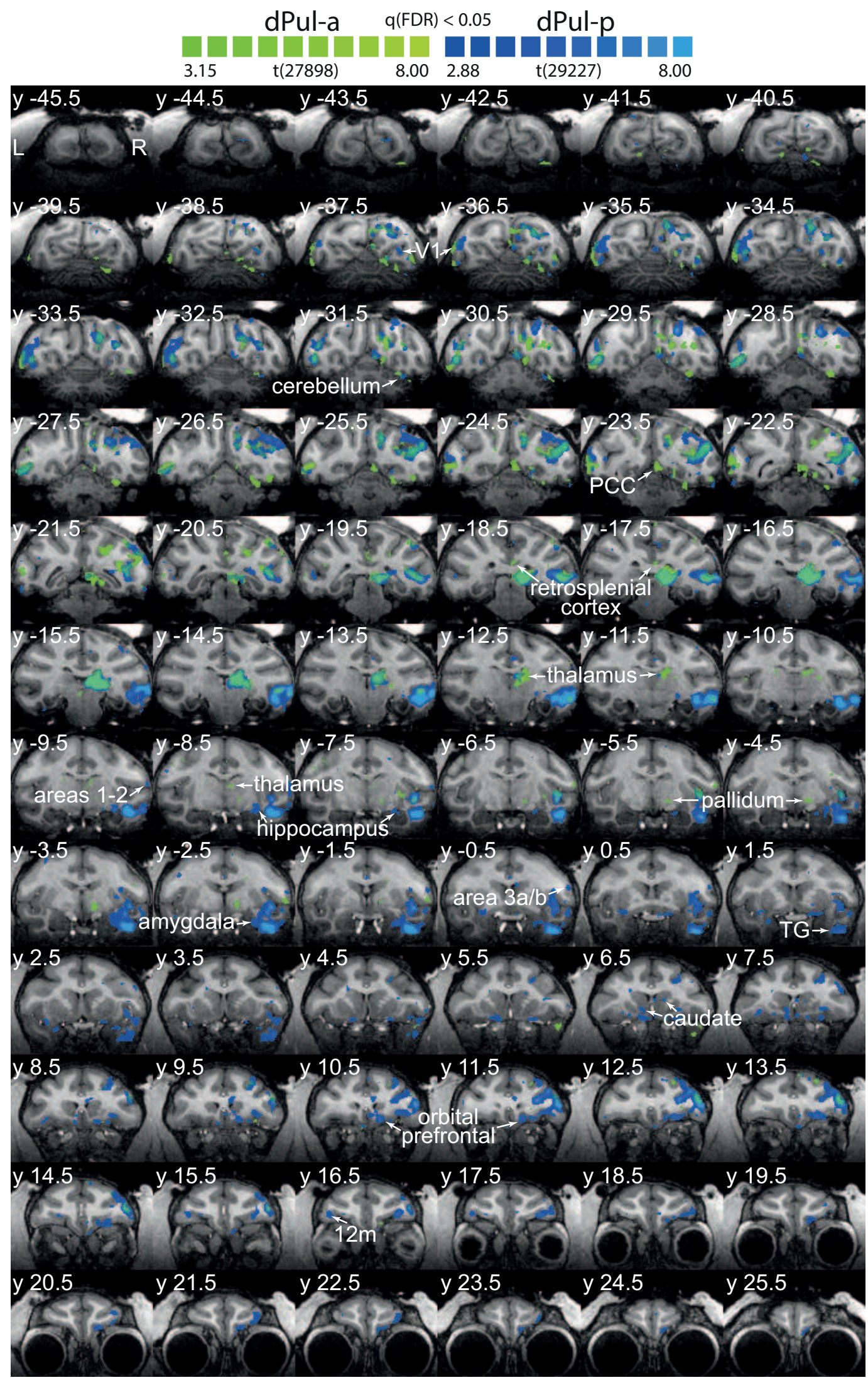

Fig. 3.19 Coronal sections showing statistical t maps of BOLD activation during unilateral high-current stimulation of dPul-a (green) and dPul-p (blue) in the right hemisphere combined across all three cognitive tasks in monkey B. L: left, R: right, $\mathrm{Y}$ : distance from AC-PC origin in the anterior/posterior plane in millimeters. 


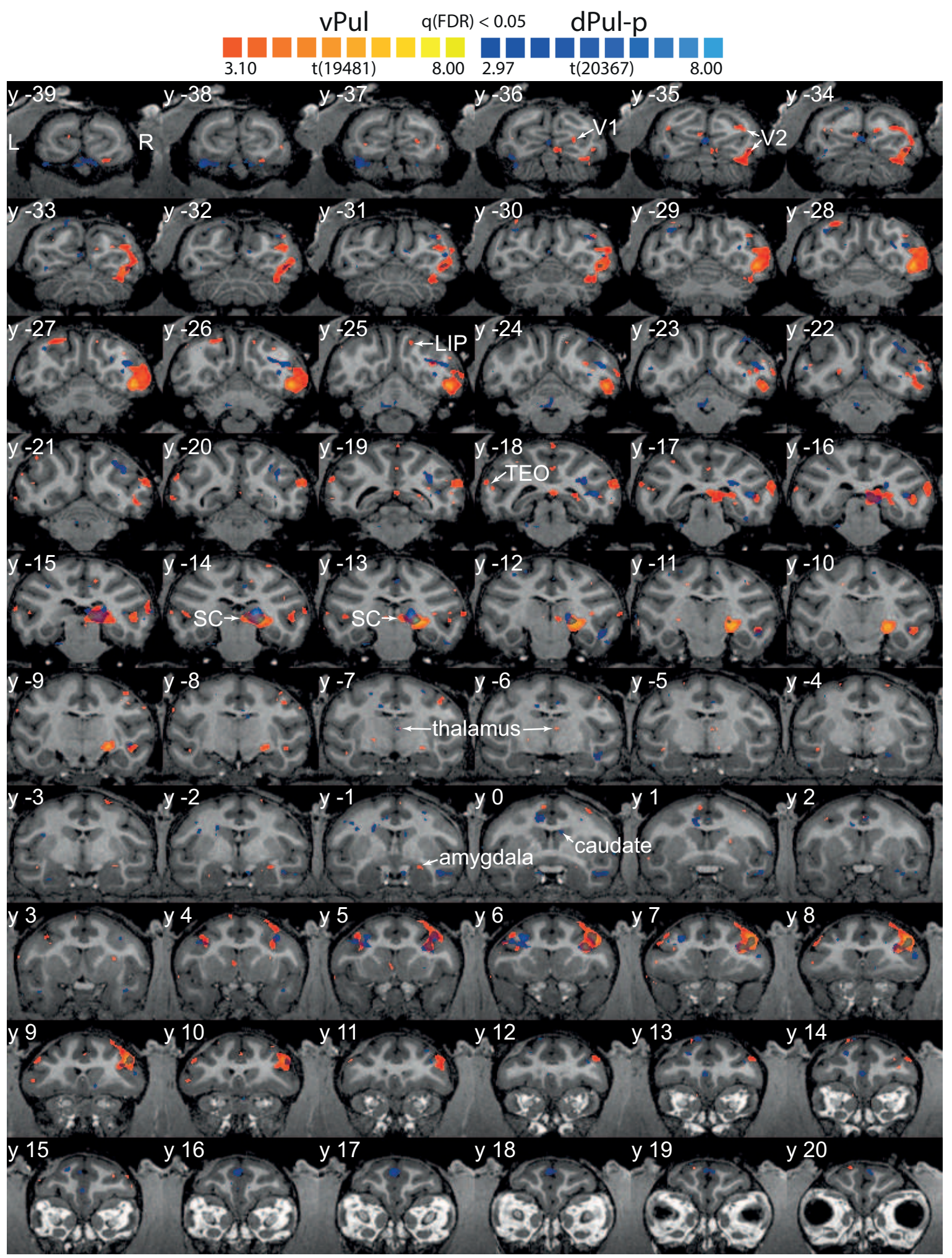

Fig. 3.20 Coronal sections showing statistical $t$ maps of BOLD activation during unilateral low-current stimulation of dPul-p (blue) and vPul (red/yellow) in the right hemisphere combined across all three cognitive tasks in monkey C. L: left, R: right, Y: distance from AC-PC origin in the anterior/posterior plane in millimeters. 


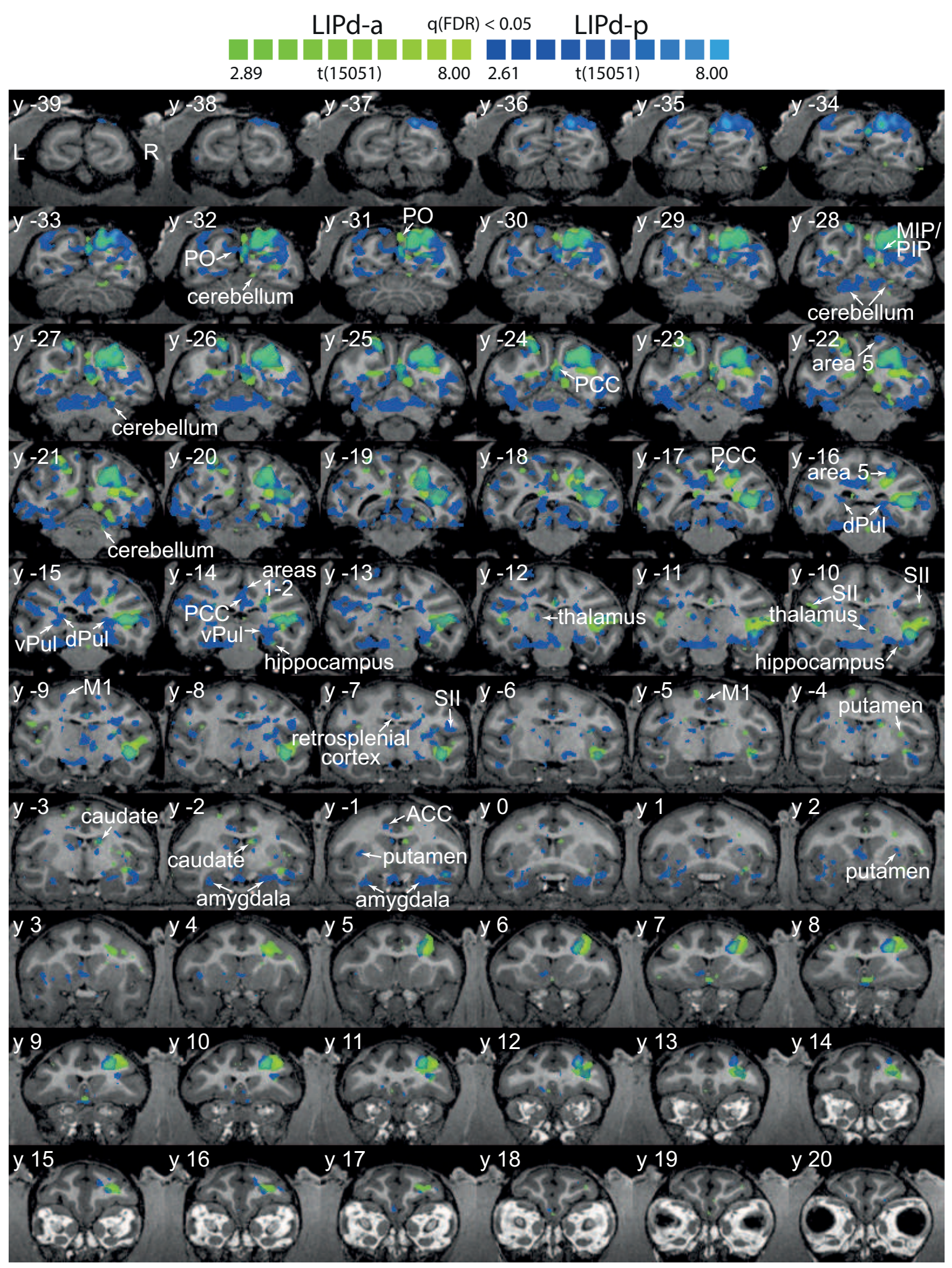

Fig. 3.21 Coronal sections showing statistical t maps of BOLD activation during unilateral high-current stimulation of LIPd-a (green) and LIPd-p (blue) in the right hemisphere combined across all three cognitive tasks in monkey C. L: left, R: right, Y: distance from AC-PC origin in the anterior/posterior plane in millimeters. 


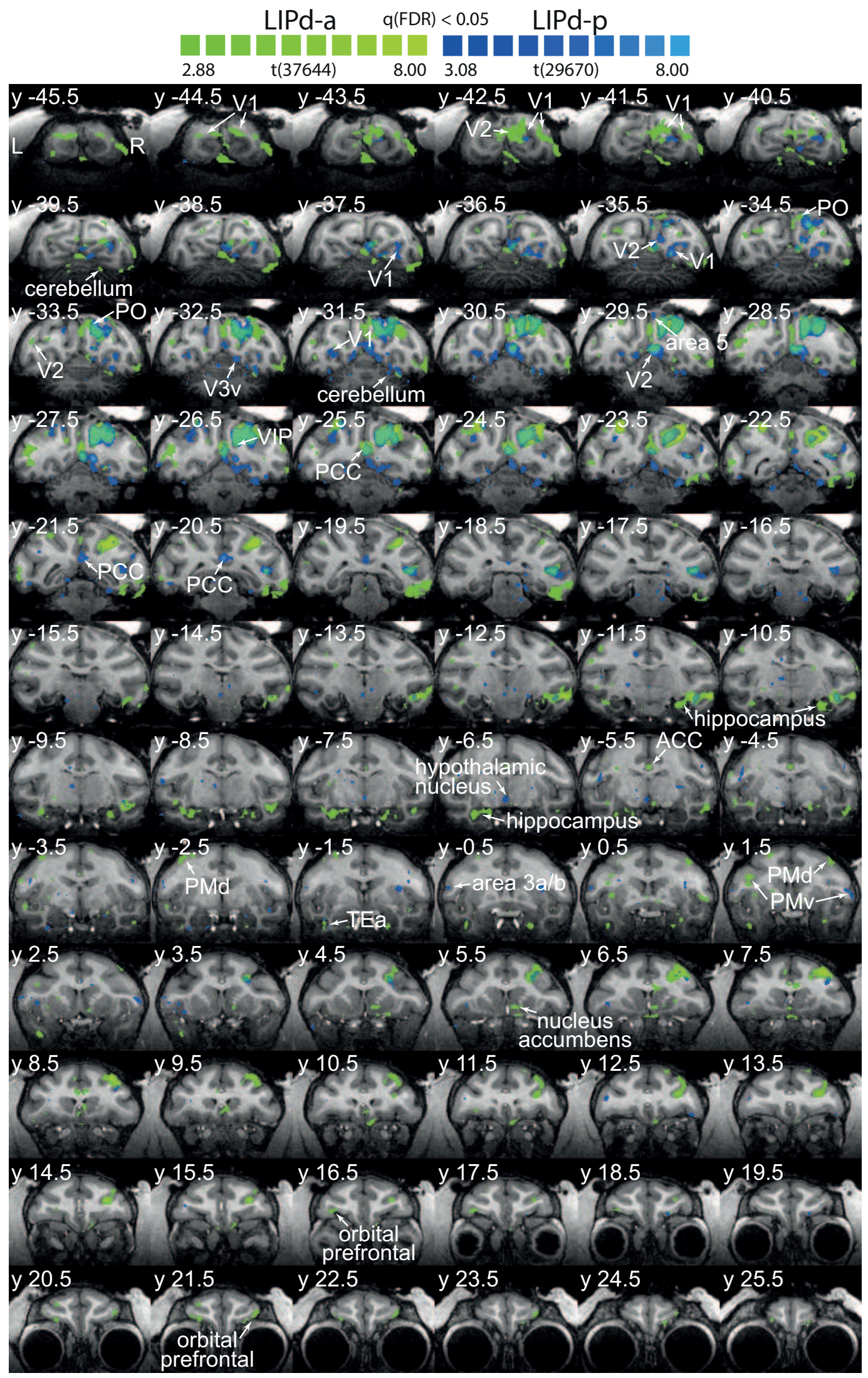

Fig. 3.22 Coronal sections showing statistical $t$ maps of BOLD activation during unilateral high-current stimulation of LIPd-a (green) and LIPd-p (blue) in the right hemisphere combined across all three cognitive tasks in monkey B. L: left, R: right, Y: distance from AC-PC origin in the anterior/posterior plane in millimeters. 
Right hemisphere (stimulated)

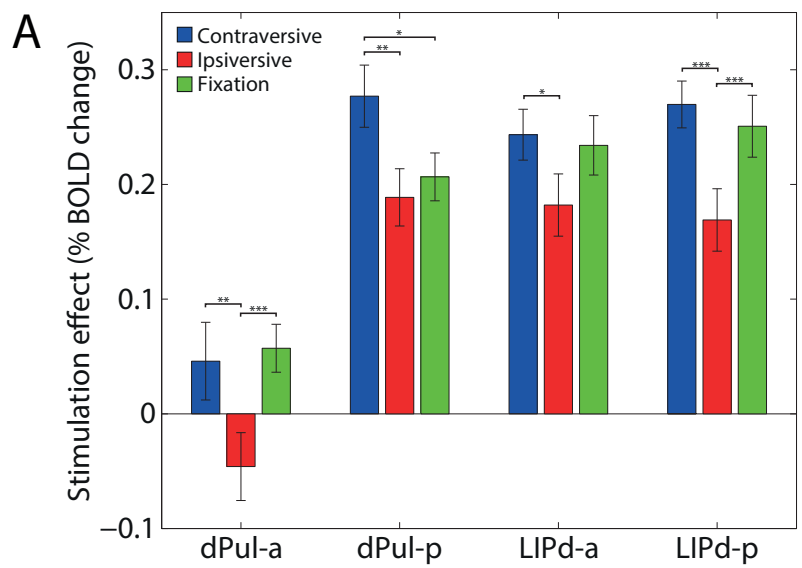

B

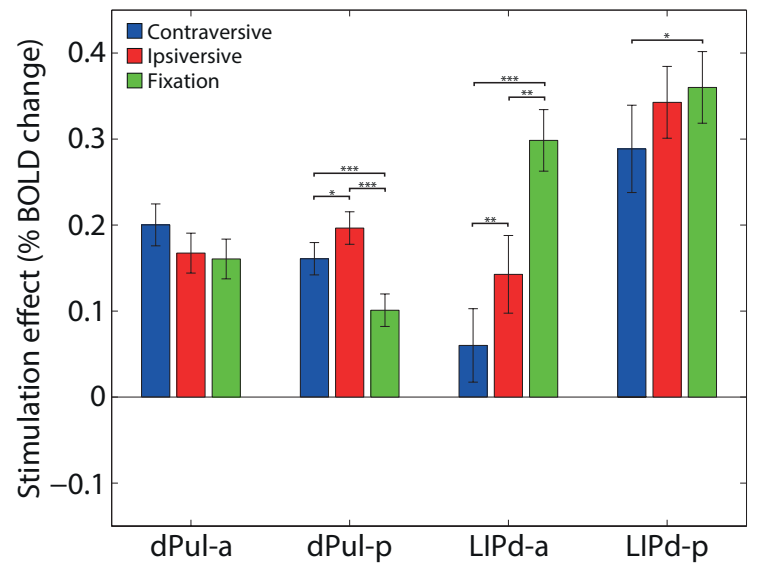

Left hemisphere
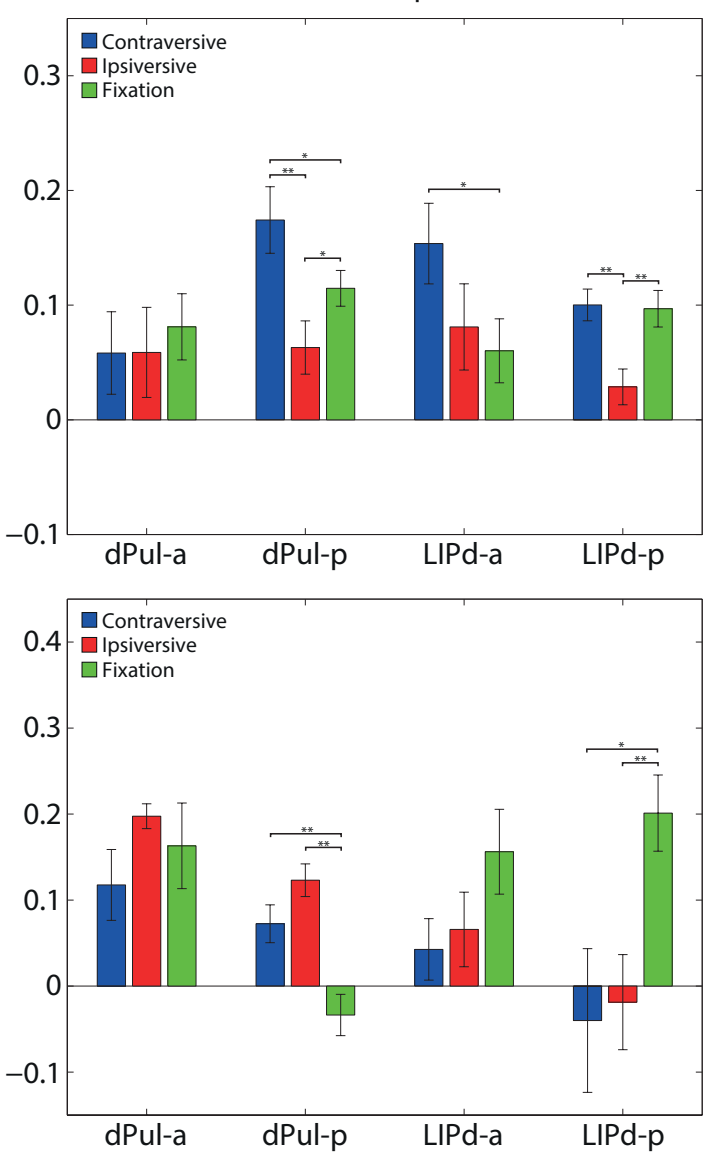

Fig. 3.23 Effects of stimulation in dPul-a, dPul-p, LIPd-a, and LIPd-p on BOLD responses in monkey $\mathrm{C}(\mathrm{A})$ and monkey $\mathrm{B}(\mathrm{B})$ in the right, stimulated hemisphere (left panel) and the left hemisphere (right panel). Stimulation effects are calculated as the difference in BOLD change between stimulation and control conditions for the contraversive (left) memory saccade task (blue bars), the ipsiversive (right) memory saccade task (red bars), and the fixation task (green bars), separately. $* \mathrm{p}<0.05, * * \mathrm{p}<0.01, * * * \mathrm{p}<0.001$.

\subsubsection{Effects of dPul and LIP microstimulation on BOLD responses during different cognitive tasks}

Table 3.3 gives an overview of ROIs defined for dPul stimulation in each animal. Fig. 3.23 shows the magnitude of the effects of microstimulation in all four stimulation sites for each task and hemisphere. In monkey $\mathrm{C}$, the rmANOVA on mean BOLD response amplitudes during dPul-a stimulation or during the corresponding time windows in control conditions across ROIs in the right, stimulated hemisphere $(n=59)$ revealed a main effect of the cognitive task $(F(2,116)=3.30, p<0.05)$ and although the task $\times$ stimulation interaction did not reach significance $(F(2,116)=0.80, p=0.4531)$, post-hoc $\mathrm{t}$ tests contrasting the stimulation 
effects, i.e. the differences between BOLD response amplitudes in stimulation and control conditions, showed that dPul-a stimulation led to a greater increase in BOLD response in the contraversive memory saccade task compared to the ipsiversive memory saccade task $(t(58)=3.35, p<0.01)$. Moreover, dPul-a stimulation increased BOLD responses more strongly in the fixation task than in the ipsiversive task $(t(58)=-4.89, p<0.001)$. For the left hemisphere $(n=23)$, the ANOVA also revealed a significant main effect of the task $(F(2$, $44)=6.46, p<0.01)$. However, neither the task $\times$ stimulation interaction effect $(F(2,44)=$ $1.37, p=0.2654$ ) nor any of the post-hoc comparisons showed significant differences in the magnitude of the stimulation effect between tasks (all $t(22) \mathrm{s} \leq 0.55$, all $p \mathrm{~s} \geq 0.5904$ ). The rmANOVA on dPul-p stimulation effects in the right, stimulated hemisphere $(n=34)$ also showed a significant main effect of task $(F(2,66)=13.51, p<0.001)$. Again, although the task $\times$ stimulation interaction did not reach significance $(F(2,66)=1.28, p=0.2840)$, t tests contrasting the stimulation effects between tasks showed that the effects of dPul-p stimulation on BOLD responses were stronger for the contraversive memory saccade task than for the ipsiversive task $(t(33)=2.94, p<0.01)$ and the fixation task $(t(33)=2.37, p<0.05)$. Similar effects were found for the opposite (left) hemisphere $(n=22)$ with a significant main effect of task revealed by the rmANOVA $(F(2,42)=29.33, p<0.001)$ and post-hoc $\mathrm{t}$ tests indicating stronger dPul-p stimulation effects on BOLD activity in the contraversive task as compared to the ipsiversive task $(t(21)=3.19, p<0.01)$ and the fixation task $(t(21)=2.20, p<0.05)$. Additionally, greater effects of dPul-p stimulation were found for the fixation task than for the ipsiversive task $(t(21)=-2.53, p<0.05)$. In monkey $\mathrm{B}$, the rmANOVA on BOLD responses in right, stimulated hemisphere ROIs $(n=40)$ during dPul-a stimulation yielded a significant task $\times$ stimulation interaction effect $(F(2,78)=21.50, p<0.001)$. For the left hemisphere $(n=4)$ a significant main effect of task found $(F(2,6)=15.27, p<0.01)$. However, further pairwise comparisons did not reveal significant differences in stimulation effects between tasks (right hemisphere: all $t(39) \mathrm{s} \leq 1.58$, all $p \mathrm{~s} \geq 0.1216$, left hemisphere: all $t(3) \mathrm{s} \leq 2.32$, all $p \mathrm{~s} \geq 0.1034)$. Note that the number of ROIs in the left hemisphere is very low. Thus, the results of the statistical tests may not be conclusive. For dPul-p stimulation in monkey B, we found a significant main effect of task $(F(2,144)=70.96, p<0.001)$ and a significant task $\times$ stimulation interaction $(F(2,144)=4.58, p<0.05)$ for BOLD responses in the right, stimulated hemisphere $(n=73)$. Further post-hoc $t$ tests revealed greater stimulation effects in the ipsiversive task than in the contraversive task $(t(72)=-2.50, p<0.05)$. Furthermore, in comparison with the fixation task dPul-p stimulation led to a stronger increase in BOLD response during the contraversive task and the ipsiversive task, respectively $(t(72)=4.17$ and $t(72)=6.83$, both $p s<0.001)$. A similar pattern was observed for ROIs in the left hemisphere $(n=12)$ with a significant main effect of task $(F(2,22)=22.10, p<0.001)$ and a significant 
task $\times$ stimulation interaction $(F(2,22)=18.16, p<0.001)$. Again, compared to the fixation task stimulation effects were stronger in the contraversive task and in the ipsiversive task $(t(11)=4.43$ and $t(11)=4.37=6.83$, both $p s<0.01)$. Stimulation effects were also greater in the contraversive task than in the ipsiversive task. However, this difference did not reach significance $(t(11)=-2.12, p=0.0576)$.

Table 3.4 gives an overview of ROIs defined for LIP stimulation in each animal. LIPd-a stimulation in monkey $\mathrm{C}$ also showed task-dependent effects on BOLD responses. For the right, stimulated hemisphere $(n=35)$, the rmANOVA revealed a significant main effect of task $(F(2,68)=14.38, p<0.001)$ and a significant task $\times$ stimulation interaction effect $(F(2,68)=19.73, p<0.001)$. Further pairwise comparisons showed that stimulation effects were stronger for the contraversive task compared to the ipsiversive task $(t(34)=2.60, p$ $<0.05)$. Greater stimulation effects were also found for the fixation task as compared to the ipsiversive task. However, this difference did not reach significance $(t(34)=-2.02, p=$ $0.0515)$. For the left hemisphere $(n=14)$, the rmANOVA also resulted in a significant main effect of task $(F(2,26)=29.17, p<0.001)$ and futher $t$ tests revealed stronger effects of LIPd-a stimulation during the contraversive than during the fixation task $(t(13)=2.23, p<$ 0.05). For LIPd-p stimulation in monkey $C$, the rmANOVA for right, stimulated hemisphere ROIs $(n=49)$ yielded a significant task $\times$ stimulation interaction effect $(F(2,96)=11.84$, $p<0.001$ ) which was driven by greater effects of LIPd-p stimulation on BOLD responses during the contraversive task than during the ipsiversive task $(t(48)=3.96, p<0.001)$ and during the fixation task compared to the ipsiversive task $(t(48)=-4.20, p<0.001)$. For left hemisphere ROIs $(n=32)$, there were significant main effects of task $(F(2,62)=15.04, p<$ $0.001)$ and stimulation $(F(1,31)=15.34, p<0.001)$ yielded by the rmANOVA. Post-hoc comparisons revealed that LIPd-p stimulation had a greater effect on BOLD responses during the contraversive task compared to the ipsiversive task $(t(31)=3.13, p<0.01)$. Moreover, there were stronger stimulation effects for the fixation task than for the ipsiversive task $(t(31)$ $=-3.45, p<0.01)$. LIPd-a stimulation in monkey $\mathrm{B}$ had significant main effects of task $(F(2$, $94)=14.12, p<0.001)$ and stimulation $(F(1,47)=16.86, p<0.001)$ and a significant task $\times$ stimulation interaction effect $(F(2,94)=17.39, p<0.001)$ in right hemisphere ROIs $(n=48)$. Further pairwise comparisons showed that LIPd-a stimulation had greater effects during the ipsiversive task than in the contraversive task $(t(47)=-3.28, p<0.01)$ and in the fixation task compared to both the contraversive and the ipsiversive task $(t(47)=-4.44, p<0.001$ and $t(47)$ $=-3.08, p<0.01$, respectively). For ROIs in the left hemisphere $(n=16)$, the rmANOVA on BOLD responses only revealed a significant main effect of task $(F(2,30)=7.17, p<0.01)$. Post-hoc $\mathrm{t}$ tests did not show significant differences in stimulation effects between tasks (all $t(15) \mathrm{s} \leq 1.78$, all $p \mathrm{~s} \geq 0.0960$ ). For LIPd-p stimulation in monkey $\mathrm{B}$, the rmANOVA on 
BOLD responses in the right, stimulated hemisphere $(n=29)$ yielded a significant main effect of task $(F(2,56)=33.43, p<0.001)$ and a significant task $\times$ stimulation interaction $(F(2,56)=3.78, p<0.05)$. Pairwise comparisons revealed that LIPd-p stimulation during the fixation task had a greater effect than during the contraversive task $(t(28)=-2.07, p<0.05)$. For left hemisphere ROIs $(n=8)$, the rmANOVA also revealed a significant main effects of task $(F(2,14)=10.76, p<0.01)$ and stimulation $(F(1,7)=17.17, p<0.01)$ and a significant task $\times$ stimulation interaction $(F(2,14)=7.94, p<0.01)$. Further pairwise comparisons showed that stimulation effects were greater in the fixation task than in the contraversive task $(t(7)=-2.94, p<0.05)$ and in the ipsiversive task $(t(7)=-4.40, p<0.01)$.

\subsubsection{Additive effects of microstimulation on BOLD responses}

In order to test whether stimulation effects on BOLD responses reflected an addition of activity to the initial BOLD responses in control conditions or rather a multiplication of that initial BOLD response by a certain factor, we fitted the stimulation effects across ROIs with an additive and a multiplicative model separately for each hemisphere. The resulting adjusted R-Squared values are shown in Fig. 3.24. In monkey C, with the only exception being BOLD responses in the ipsiversive memory saccade task during dPul-a stimulation,
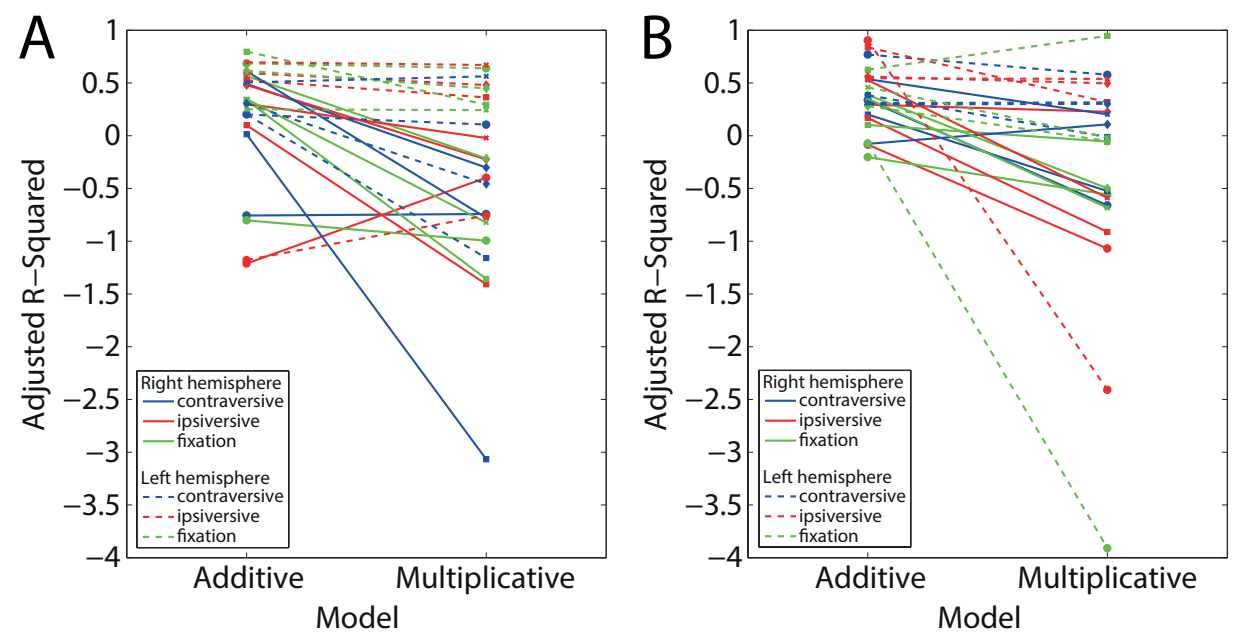

Fig. 3.24 Goodness of fit for modeling effects of microstimulation of dPula (dots), dPul-p (squares), LIPd-a (diamonds), and LIPd-p (X's) in monkey C (A) and in monkey B (B) using an additive or a multiplicative model. Adjusted R-Squared values are shown for both models applied separately on BOLD responses across ROIs in the right, stimulated hemisphere (solid lines) and in the left hemisphere (dashed lines). Blue: contraversive memory saccade task, red: ipsiversive memory saccade task, green: fixation task. 

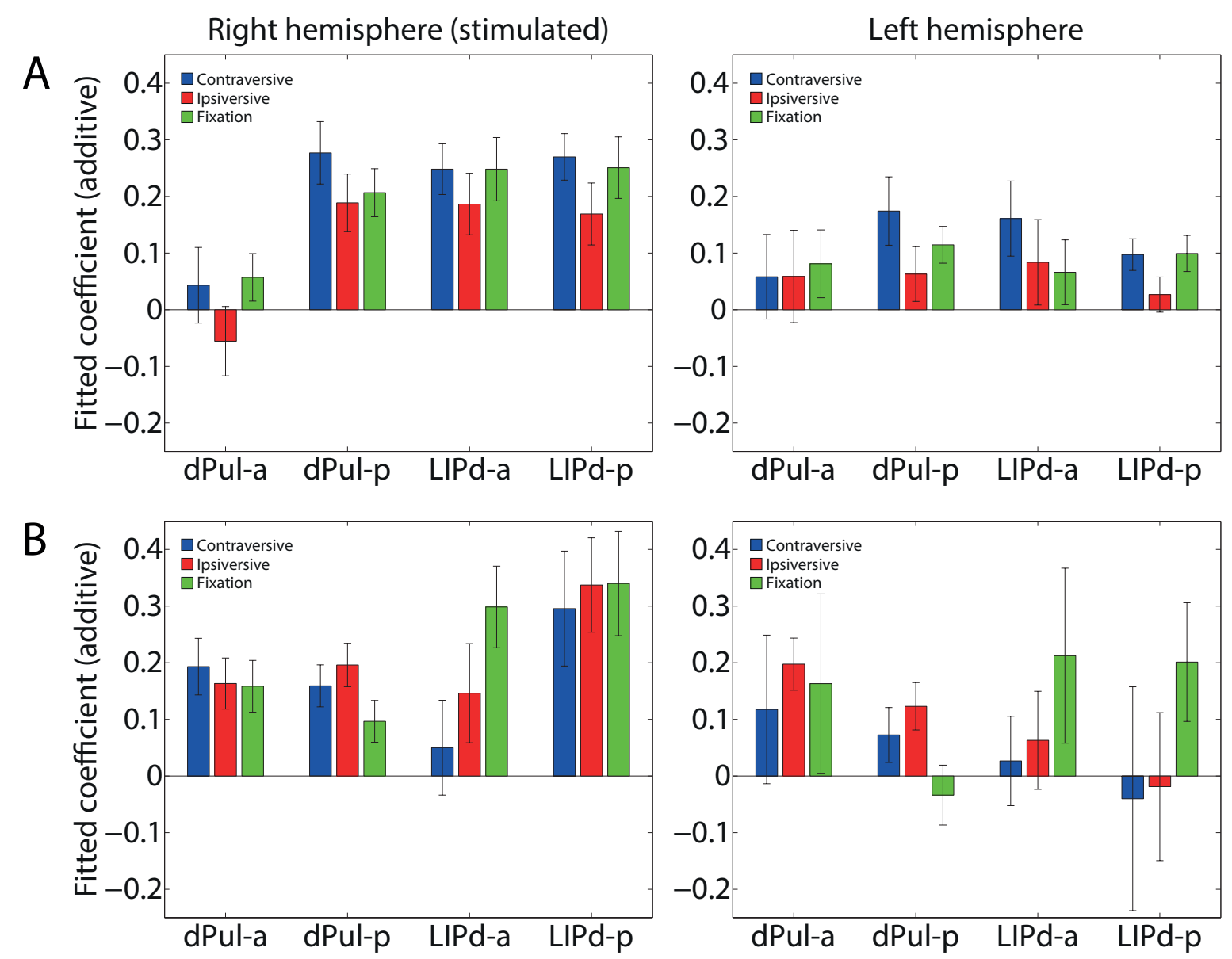

Fig. 3.25 Fitted coefficients with root-mean-square errors resulting from modeling effects of microstimulation of dPul-a, dPul-p, LIPd-a, and LIPd-p on BOLD responses across ROIs in the right, stimulated hemisphere (left panel) and in the left hemisphere (right panel) of monkey $\mathrm{C}$ (A) and monkey $\mathrm{B}$ (B) using an additive model. Blue: contraversive memory saccade task, red: ipsiversive memory saccade task, green: fixation task.

the additive model either fitted the BOLD responses as well as the multiplicative model or the additive model was superior to the multiplicative model as reflected by similar or higher adjusted R-Squared values, respectively. A similar pattern was found in monkey B with the only exceptional cases where higher adjusted R-Squared values were found for the multiplicative model being stimulation effects in the left hemisphere in the fixation task during dPul-p stimulation and stimulation effects in the right hemisphere in the contraversive memory saccade task during LIPd-a stimulation. Although variability in the fitted coefficients resulting from modeling stimulation effects with the additive model was higher than in the actual BOLD stimulation effects, the fitted coefficients nicely resemble the actual magnitude of stimulation effects on BOLD responses (compare fitted coefficients in Fig. 3.25 with actual BOLD stimulation effects shown in Fig. 3.23). 


\subsubsection{Changes in contraversive selectivity during dPul and LIP micros- timulation}

In order to investigate whether microstimulation of dPul and LIP changes space representations in the brain, we tested the contraversive selectivity across ROIs in control conditions against contraversive selectivity in stimulation conditions. In Fig. 3.26, the CSI in control conditions is plotted against the CSI in stimulation conditions for each ROI and hemisphere in monkey C. ROIs that lie above the line of equality show a higher CSI and thus stronger contraversive selectivity during stimulation than during control conditions. ROIs that lie below the line of equality show a lower CSI and thus weaker contraversive selectivity during stimulation than during control. As can be seen in Fig. 3.26A and B, both dPul-a and dPul-p stimulation in monkey $\mathrm{C}$ led to higher CSIs and thus stronger contraversive selectivity across ROIs in the right hemisphere $(t(59)=-3.55, p<0.001$ and $t(33)=-2.94, p<0.01$, respectively). dPul-p stimulation also increased contraversive selectivity in the left hemisphere $(t(21)=-3.19, p<0.01)$. In monkey B, however, dPul stimulation did not have such consistent effects on contraversive selectivity. As shown in Fig. 3.27A, dPul-a stimulation did not significantly change contraversive selectivity in either of the two hemispheres $(t(40)$ $=-1.29, p=0.2041$ and $t(3)=2.32, p=0.1030$ ). Note that the number of ROIs in the left hemisphere may be too low to yield conclusive results across ROIs. However, dPul-p stimulation significantly changed contraversive selectivity in the right, stimulated hemisphere (see Fig. 3.27B) but, in contrast to the pattern found in monkey C, lower CSIs, i.e. weaker contraversive selectivity, was found during stimulation than during control conditions $(t(73)=$ $2.43, p<0.05)$. A similar pattern was found for ROIs in the left hemisphere but the difference between CSIs did not reach significance $(t(11)=2.12, p=0.0576)$.

LIP stimulation in monkey $\mathrm{C}$ led to similar changes in contraversive selectivity as dPul stimulation (see Fig. 3.26C and D). Both LIPd-a and LIPd-p stimulation led to greater CSIs in right hemisphere ROIs in monkey $\mathrm{C}(t(34)=-2.60, p<0.05$ and $t(48)=-3.96, p<0.001)$. In the left hemisphere greater CSIs were found during LIPd-p stimulation than in control conditions $(t(31)=-3.13, p<0.01)$. For LIPd-a stimulation the difference between CSIs during stimulation and control did not reach significance $(t(14)=-1.95, p=0.0715)$. In monkey B, however, only LIPd-a stimulation led to changes in contraversive selectivity in right hemisphere ROIs. Unlike LIP stimulation effects in monkey $\mathrm{C}$, in monkey B CSIs were greater in control conditions than during LIPd-a stimulation $(t(49)=3.70, p<0.001)$. Contraversive selectivity in the left hemisphere was not significantly changed during LIPd-a stimulation $(t(16)=0.92, p=0.3721)$. LIPd-p stimulation did not significantly affect CSIs in either of the two hemispheres $(t(28)=1.24, p=0.2258$ and $t(7)=0.27, p=0.7941$, respectively). 
Right hemisphere (stimulated)
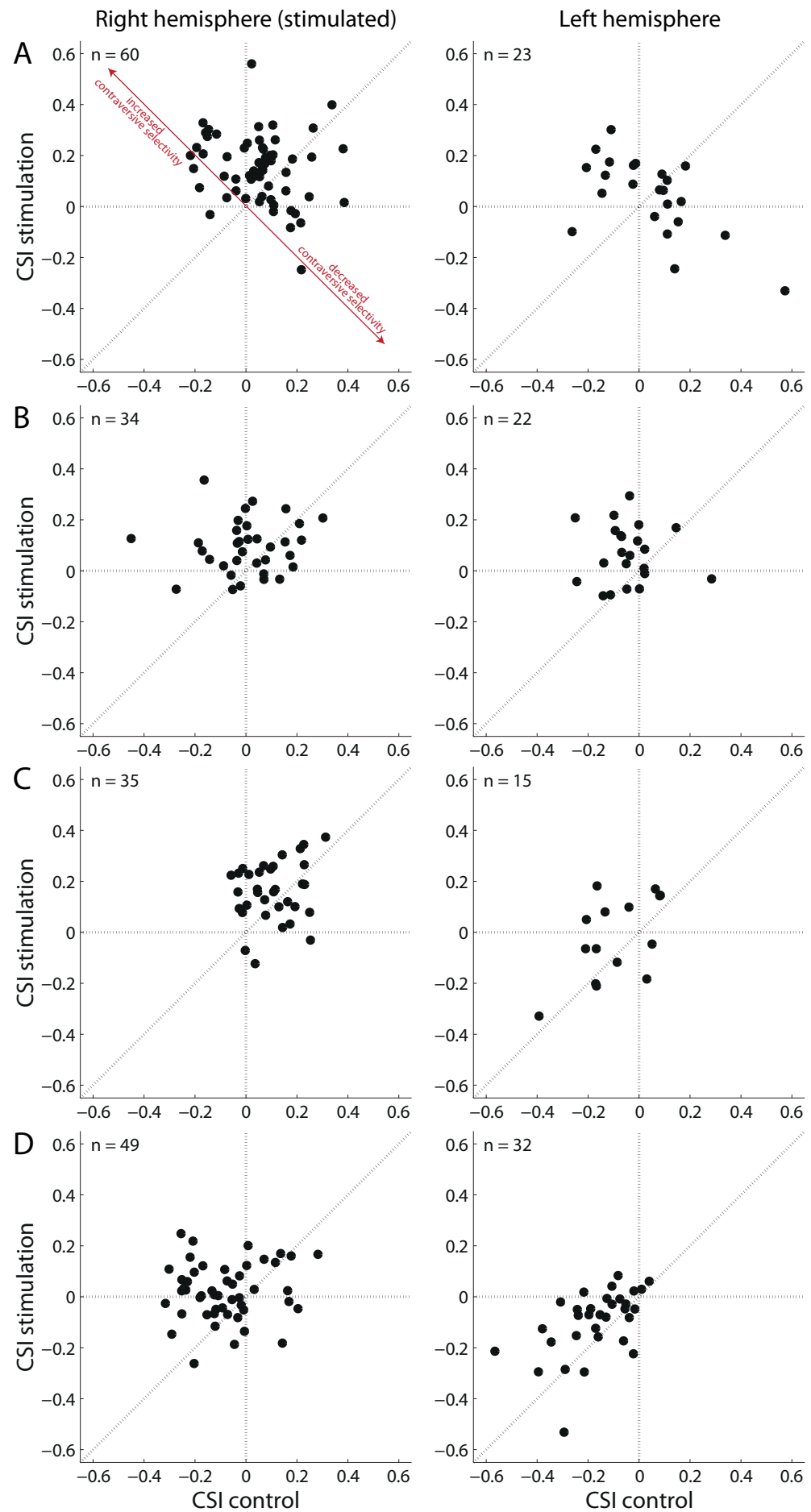

Fig. 3.26 Effects of microstimulation in (A) dPul-a, (B) dPul-p, (C) LIPd-a, (D) LIPd-p on contraversive selectivity in the right, stimulated hemisphere (left panel) and the left hemisphere (right panel) in monkey C. For each ROI, the contraversive selectivity index (CSI) in control trials is plotted against the CSI in stimulation trials. ROIs above the line of equality show an increase in contraversive tuning in stimulation trials. ROIs below the line of equality show a decrease in contraversive tuning in stimulation trials. 

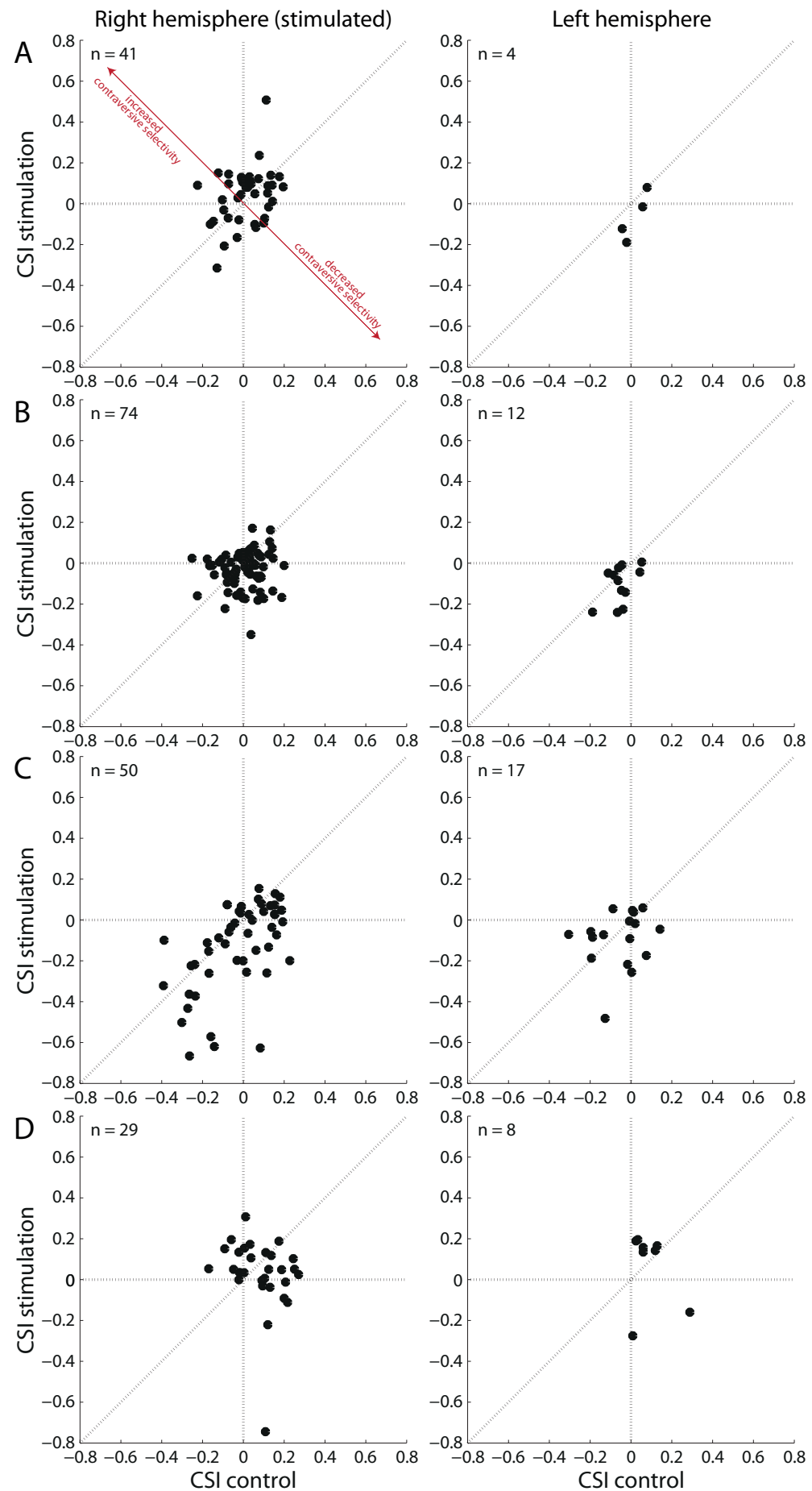

Fig. 3.27 Effects of microstimulation in (A) dPul-a, (B) dPul-p, (C) LIPd-a, (D) LIPd-p on contraversive selectivity in the right, stimulated hemisphere (left panel) and the left hemisphere (right panel) in monkey B. Same conventions as in Fig. 3.26. 


\subsubsection{Relationship between contraversive selectivity and the magni- tude of stimulation effects on BOLD responses}

To assess whether the magnitude of stimulation effects on BOLD responses in the three cognitive tasks depended on the contraversive selectivity of the activated ROIs, we first correlated the CSI values of all ROIs in control conditions with the differences between BOLD responses in stimulation and control conditions for each task separately. The resulting correlations for $\mathrm{dPul}$ stimulation in monkey $\mathrm{C}$ are shown as scatterplots with least-square lines in Fig. 3.28A and B. Effects of both dPul-a and dPul-p stimulation were significantly correlated with control CSI values. More specifically, we found a negative correlation for stimulation effects in the contraversive memory saccade task $(r=-0.53, p<0.001$ and $r=$ $-0.49, p<0.001)$ and a positive correlation for the ipsiversive memory saccade task $(r=0.35, p$ $<0.01$ and $r=0.38, p<0.01$ ). That is, lower CSI values, i.e. weaker contraversive selectivity was associated with a stronger enhancement of BOLD responses in the contraversive task. In contrast, in the ipsiversive task BOLD responses were more strongly enhanced with higher CSI values, i.e. stronger contraversive selectivity. Additionally, dPul-p stimulation effects in the fixation task were also positively correlated with CSI values $(r=0.31, p<$ $0.05)$. In order to confirm that the magnitude of stimulation effects was different depending on the strength of contraversive selectivity of an ROI, we grouped ROIs into ROIs with contraversive tuning (CSI values greater than 0 ) an ROIs with ipsiversive tuning (CSI values lower than 0) and tested whether the magnitude of stimulation effects differed between tasks within each group of ROIs using a mixed ANOVA. For both dPul-a and dPul-p stimulation significant main effects of task $(F(2,162)=16.16$ and $F(2,108)=11.42$, both $p$ s $<0.001)$ and significant contraversive selectivity $\times$ task interaction effects $(F(2,162)=25.06$ and $F(2,108)=23.65$, both $p$ s $<0.001)$ were found. Further post-hoc tests showed that, for contraversively tuned ROIs, dPul-a stimulation had a stronger effect during the fixation task than during the contraversive memory saccade task $(n=55, t(54)=-3.30, p>0.01)$ whereas effects of both dPul-a and dPul-p stimulation were stronger during the contraversive task than during the fixation task in ipsiversively tuned ROIs $(n=28, t(27)=4.59, p>0.001$ and $n=33, t(32)=6.42, p<0.001)$. Furthermore, in ROIs with ipsiversive tuning both dPul-a and dPul-p stimulation had greater effects in the contraversive task than in the ipsiversive task $(t(27)=11.10, p>0.001$ and $t(32)=7.27, p<0.001)$ and stimulation effects in the fixation task were stronger than in the ipsiversive task $(t(27)=-4.63, p>0.001$ and $t(32)=$ $-2.06, p<0.05)$.

In monkey $\mathrm{B}, \mathrm{dPul}-\mathrm{a}$ stimulation effects did not significantly correlate with either of the three tasks $(-0.10 \leq$ all $r \mathrm{~s} \leq 0.26$, all $p \mathrm{~s} \geq 0.1036)$. For dPul-p stimulation, however, there were positive correlations between the stimulation effects and the CSI values in the 
ipsiversive and in the fixation task $(r=0.52, p<0.001$ and $r=0.27, p<0.05)$. Moreover, the ANOVA on BOLD responses during dPul-p stimulation revealed significant main effects of contraversive selectivity and task and a significant contraversive selectivity $\times$ task interaction $(F(1,84)=12.49, F(2,168)=32.96$, and $F(2,168)=7.62$, all $p$ s $<0.001)$. Pairwise comparisons showed that, for ROIs with contraversive tuning $(n=43)$, stimulation effects were stronger in the ipsiversive task compared to the contraversive task $(t(42)=-4.98, p<$ $0.001)$ and the fixation task $(t(42)=6.29, p<0.001)$. Additionally, stimulation enhanced BOLD responses more strongly in the contraversive task than in the fixation task $(t(42)=$ $2.29, p<0.05)$. For ROIs with ipsiversive tuning $(n=43)$, stimulation effects in both the contraversive and the ipsiversive task were greater than in the fixation task $(t(42)=4.68$ and $t(42)=4.28$, both $p s<0.001)$.

In monkey $\mathrm{C}$, LIP stimulation effects were similarly related to contraversive selectivity as the effects of dPul stimulation. LIPd-a stimulation effects were positively correlated with CSI values in the ipsiversive task $(r=0.36, p<0.05)$. A positive but not statistically significant correlation was also found for the fixation task $(r=0.26, p=0.0672)$. The mixed ANOVA on LIPd-a stimulation effects revealed a significant main effect of task $(F(2,96)=$ $7.43, p<0.01)$ and a significant contraversive selectivity $\times$ task interaction $(F(2,96)=3.29$, $p<0.05)$. Post-hoc comparisons between stimulation effects in ROIs with contraversive tuning ( $n=33$ ) did not show significant differences between tasks (all $t(32) \mathrm{s} \leq 1.22$, all $p \mathrm{~s}$ $\geq 0.2327)$. For ipsiversively tuned ROIs $(n=17)$, however, effects of LIPd-a stimulation were stronger in the contraversive task than in the ipsiversive task $(t(16)=4.38, p<0.001)$. Stimulation effects were also stronger in the fixation task than in the ipsiversive task but this difference did not reach significance $(t(16)=-2.01, p=0.0615)$. Like dPul-p stimulation effects, LIPd-p stimulation effects were positively correlated with CSI values in both the ipsiversive and the fixation task $(r=0.58$ and $r=0.37$, both $p \mathrm{~s}<0.001)$. Moreover, the ANOVA on stimulation effects yielded significant main effects of contraversive selectivity and task $(F(1,79)=9.37$ and $F(2,158)=6.52$, both $p$ s $<0.01)$ and a significant contraversive selectivity $\times$ task interaction effect $(F(2,158)=9.23, p<0.001)$. Similar to the pattern found for dPul stimulation, in contraversively tuned ROIs $(n=14)$, LIPd-p stimulation had stronger effects in the fixation task than in the contraversive task $(t(13)=-2.54, p<0.05)$. Although the difference did not reach significance, stimulation effects in the fixation task also tended to be stronger than in the ipsiversive task $(t(13)=-1.79, p=0.0975)$. In contrast, in ROIs with ipsiversive tuning $(n=67)$ LIPd-p stimulation effects in the contraversive task were stronger than in the fixation task $(t(66)=2.32, p<0.05)$. Moreover, stimulation led to greater enhancement of BOLD responses in the contraversive task compared to the ipsiversive task 
and stimulation effects in the fixation task were greater than in the ipsiversive task $(t(66)=$ 6.30 and $t(66)=-5.17$, both $p s<0.001)$.

In monkey B, there were positive correlations between LIPd-a stimulation effects and CSI values in the contraversive as well as in the ipsiversive memory saccade task $(r=0.50$ and $r$ $=0.67$, both $p \mathrm{~s}<0.001)$. A positive but not statistically significant correlation was also found for the fixation task ( $r=0.24, p=0.0534)$. The ANOVA on LIPd-a stimulation effects yielded significant main effects of contraversive selectivity $(F(1,65)=8.41, p<0.01)$ and task $(F(2$, $130)=20.81, p<0.001)$ and a significant contraversive selectivity $\times$ task interaction effect $(F(2,130)=12.29, p<0.001)$. Further post-hoc $t$ tests on stimulation effects in ROIs with contraversive tuning $(n=30)$ revealed greater effects of LIPd-a stimulation in both the ipsiversive task and the fixation task as compared to the contraversive task $(t(29)=-4.72, p<$ 0.001 and $t(29)=-2.29, p<0.05)$. In ipsiversively tuned ROIs $(n=37)$, stimulation effects in the fixation task were stronger than in both the contraversive and the ipsiversive task $(t(36)$ $=-4.93$ and $t(36)=-5.27$, both $p \mathrm{~s}<0.001)$. For LIPd-p stimulation, we found a positive correlation between stimulation effects and the CSI values for the ipsiversive task $(r=0.43, p$ $<0.01)$ and the ANOVA on stimulation effects revealed a significant contraversive selectivity $\times$ task interaction effect $(F(2,70)=5.17, p<0.01)$. For contraversively tuned ROIs $(n=30)$, subsequent post-hoc analysis showed that stimulation effects in both the ipsiversive task and the fixation task were stronger as compared to the contraversive task $(t(29)=-2.22, p<0.05$ and $t(29)=-3.83, p<0.001)$. By contrast, in ROIs with ipsiversive tuning $(n=7)$ LIPd-p stimulation effects were stronger in the contraversive task than in the ipsiversive task $(t(6)$ $=4.20, p<0.01)$. Moreover, greater stimulation effects were found in the fixation task as compared to the ipsiversive task $(t(6)=-4.88, p<0.01)$.

In summary, we consistently found positive correlations between the effects of microstimulation of both $\mathrm{dPul}$ and LIP and the extent of contraversive selectivity across ROIs in the ipsiversive memory saccade task. Positive correlations were also found for the fixation task, especially for the effects of dPul stimulation. However, a similar trend could be observed for stimulation in LIP. That is, stronger contraversive tuning of the respective ROIs was associated with a stronger enhancement of BOLD responses to visual cues and during motor preparation directed towards locations in the ipsiversive hemifield. Similarly, the enhancement of BOLD responses in the fixation task tended to be stronger with increasing contraversive tuning. Correlations between the magnitude of stimulation effects and the CSI values were weaker for the contraversive task, particularly in monkey B. In monkey C, however, we found negative correlations between stimulation effects and CSI values in the contraversive task for both dPul-a and dPul-p stimulation. That is, in contrast to the pattern 
found for the ipsiversive and the fixation task, dPul stimulation in the contraversive task led to stronger enhancement of BOLD responses in ROIs with weaker contraversive tuning.

We also found consistent interaction effects on stimulation effects between the extent of contraversive tuning in the respective ROIs and the task, with the only exception being dPul-a stimulation in monkey $\mathrm{B}$, which further indicates that the stimulation effects in all three cognitive tasks were different between ROIs with contraversive tuning and ROIs with ipsiversive tuning. More specifically, in ROIs with ipsiversive tuning in monkey $\mathrm{C}$, both dPul and LIP stimulation most strongly enhanced BOLD responses in the contraversive task and exhibited weakest effects in the ipsiversive task. Additionally, stimulation effects in the contraversive task were stronger than in the fixation task. In monkey B, however, the relationship between stimulation effects in the different tasks and contraversive selectivity of the respective ROIs was less consistent across stimulation sites with similarly strong effects of dPul-p stimulation in the contraversive and the ipsiversive task in ROIs with ipsiversive tuning. For LIPd-a stimulation the strongest effects in ROIs with ipsiversive tuning were found for the fixation task with, again, similar effects in the contraversive and the ipsiversive task. For ROIs with contraversive tuning in monkey $\mathrm{C}$, differences between the stimulation effects in the different tasks were smaller but yielded stronger effects of both dPul and LIP stimulation in the fixation task as compared to the contraversive task. In contraversively tuned ROIs in monkey B, however, both $\mathrm{dPul}$ and LIP stimulation effects were stronger in the ipsiversive task than in the contraversive task. Taken together, our results indicate reversed patterns of task-dependent effects of dPul and LIP stimulation for ROIs with contraversive tuning and ROIs with ipsiversive tuning. 

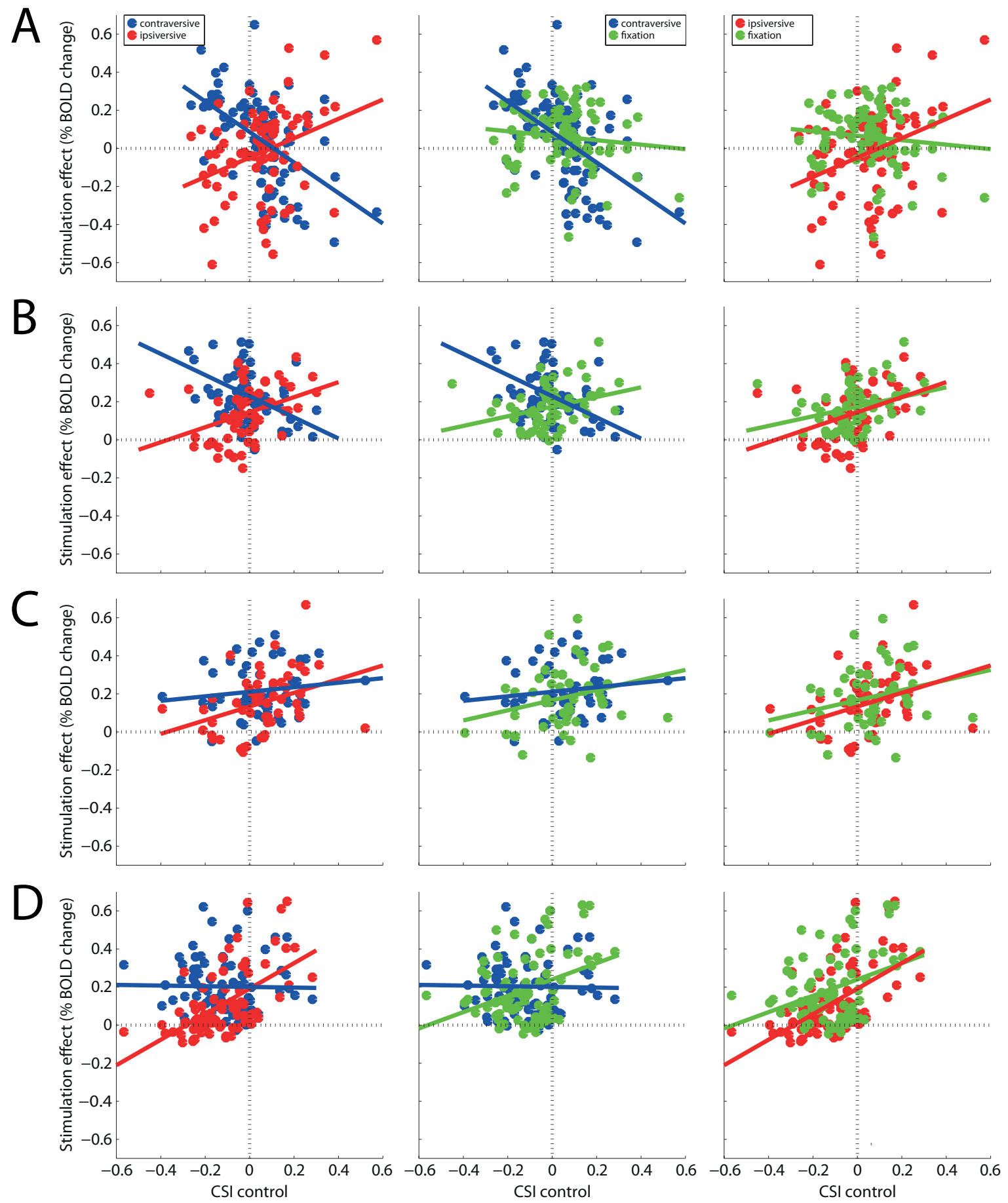

Fig. 3.28 Effects of microstimulation in (A) dPul-a, (B) dPul-p, (C) LIPd-a, (D) LIPd-p on BOLD response per ROI (both hemispheres) in monkey C. For each ROI, the effect of stimulation, defined as the difference between the BOLD response in stimulation and control conditions, is plotted against its contraversive selectivity index (CSI) in control conditions for each cognitive task. Colored dots represent ROIs, colored solid lines show linear fits of stimulation effects across ROIs. Blue: contraversive (left) memory saccade task, red: ipsiversive (right) memory saccade task, green: fixation task. 

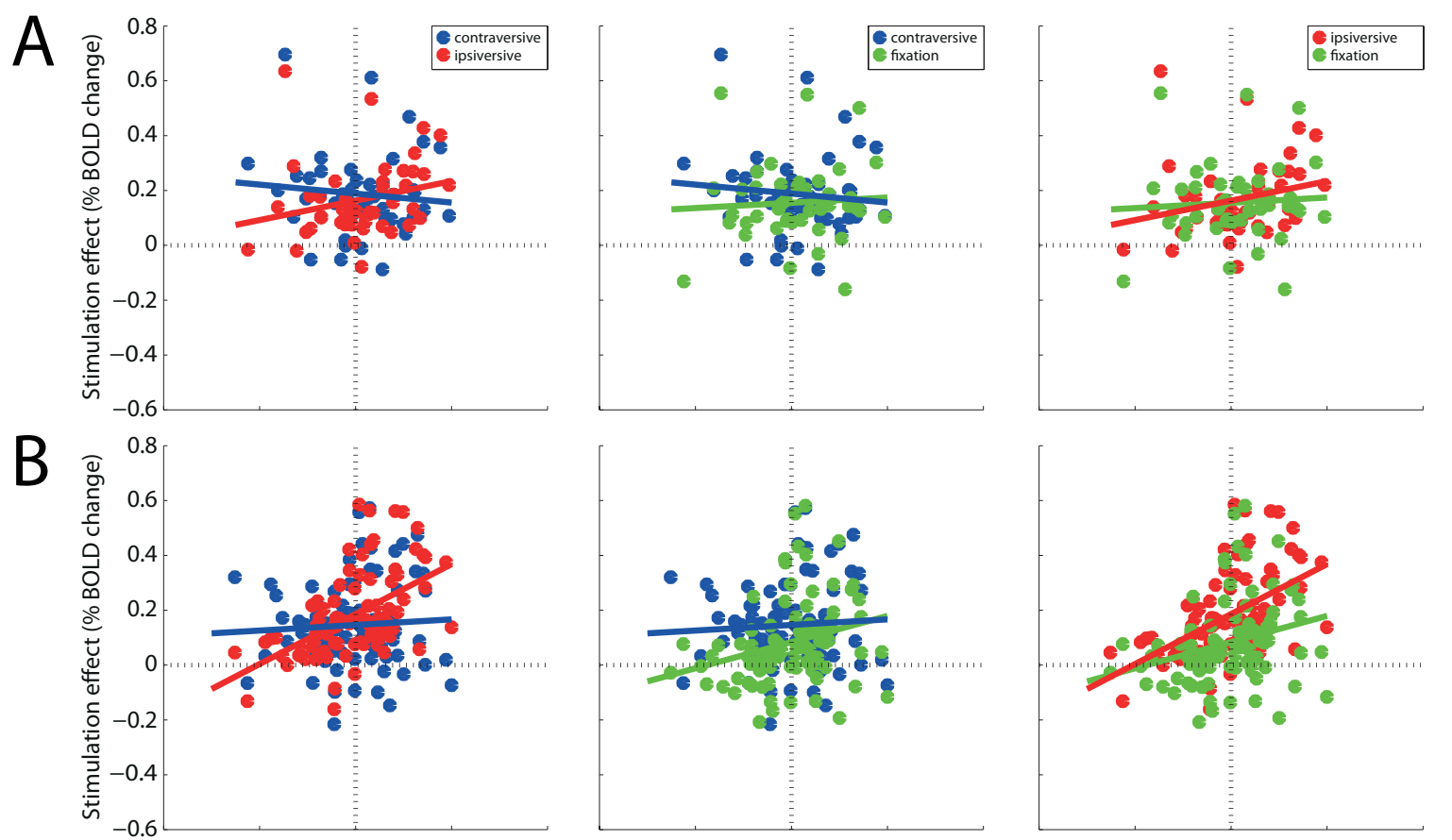

C
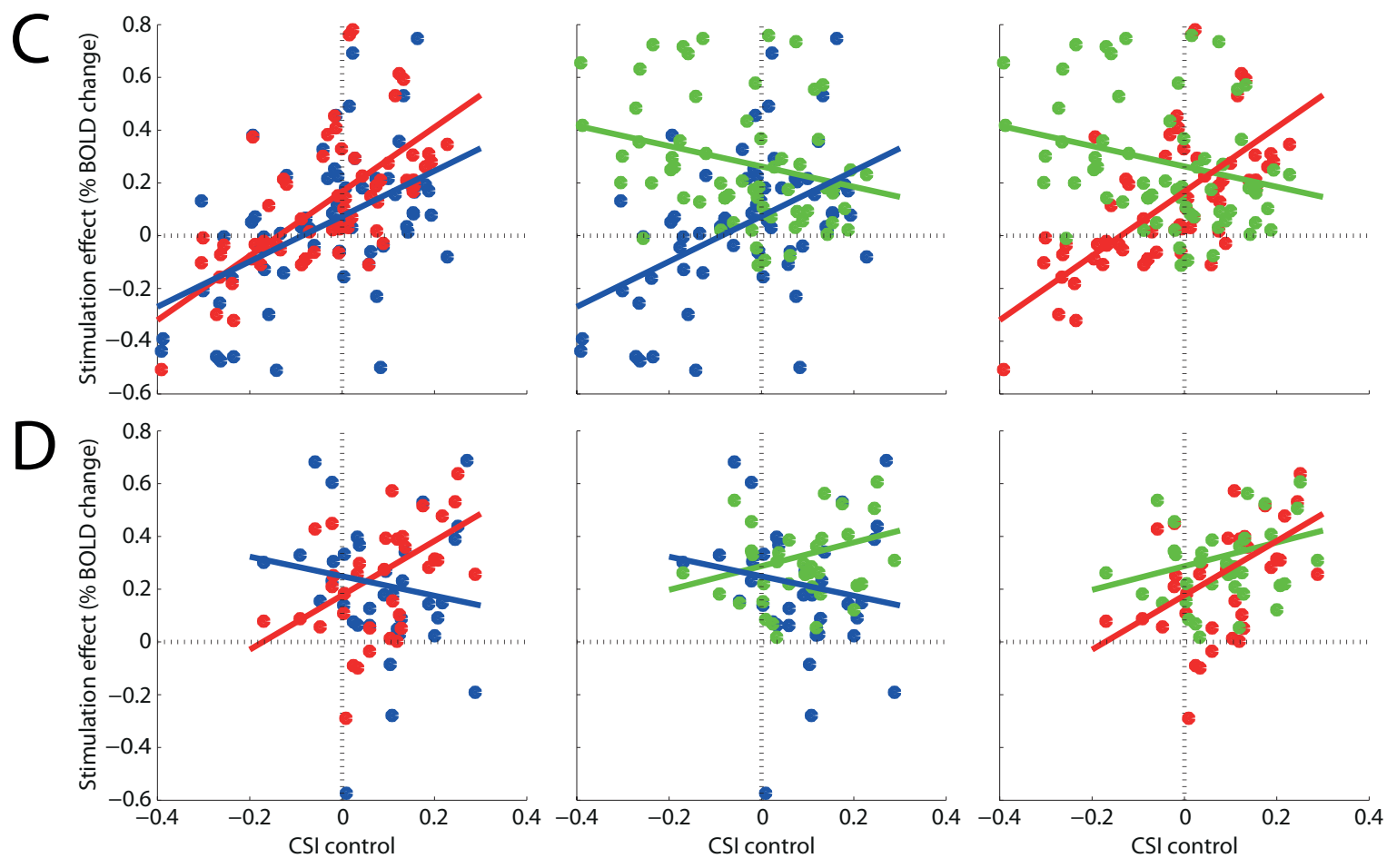

Fig. 3.29 Effects of microstimulation in (A) dPul-a, (B) dPul-p, (C) LIPd-a, (D) LIPd-p on BOLD response per ROI (both hemispheres) in monkey B. Same conventions as in Fig. 3.28. 
Table 3.3 Regions of interest (ROIs) defined for high-current stimulation in dPul with coordinates of ROI peak voxels ([x, $\mathrm{y}, \mathrm{z}]$ ) as distance from AC-PC origin in millimeters and total number of voxels per ROI.

\begin{tabular}{|c|c|c|c|c|c|c|c|c|}
\hline \multirow{3}{*}{ ROI } & \multicolumn{4}{|c|}{ dPul-a } & \multicolumn{4}{|c|}{ dPul-p } \\
\hline & \multicolumn{2}{|c|}{ Monkey C } & \multicolumn{2}{|c|}{ Monkey B } & \multicolumn{2}{|c|}{ Monkey C } & \multicolumn{2}{|c|}{ Monkey B } \\
\hline & Peak voxel & Nr. of voxels & Peak voxel & Nr. of voxels & Peak voxel & Nr. of voxels & Peak voxel & Nr. of voxels \\
\hline \multicolumn{9}{|c|}{ Right hemisphere } \\
\hline Frontal lobe & & & & & & & & \\
\hline 8B & & & {$[10,13,16]$} & 75 & & & & \\
\hline $8 \mathrm{Bs}$ & {$[10,12,15]$} & 165 & & & & & {$[12,8,14]$} & 282 \\
\hline 111 & & & & & & & {$[14,22,6]$} & 201 \\
\hline $11 \mathrm{~m} / 13 \mathrm{~b}$ & & & & & & & {$[5,23,1]$} & 149 \\
\hline $12 \mathrm{~m}$ & & & & & & & {$[15,17,5]$} & 245 \\
\hline 120 & & & & & & & {$[19,12,2]$} & 165 \\
\hline $12 \mathrm{r}$ & & & & & & & {$[15,22,8]$} & 204 \\
\hline $13 b$ & & & & & & & {$[5,11,-1]$} & 244 \\
\hline 131 & & & & & & & {$[15,10,2]$} & 196 \\
\hline 131-a & & & & & & & {$[11,13,1]$} & 258 \\
\hline a44 & {$[15,9,7]$} & 170 & & & {$[14,5,10]$} & 288 & & \\
\hline a44-d & {$[14,5,9]$} & 165 & & & & & & \\
\hline a45 & & & & & {$[19,10,10]$} & 315 & & \\
\hline $\mathrm{a} 45-\mathrm{a}$ & & & {$[20,14,8]$} & 223 & & & {$[19,15,7]$} & 264 \\
\hline$a 45-p$ & & & {$[22,9,8]$} & 76 & & & {$[21,12,6]$} & 336 \\
\hline a45-1 & {$[19,10,10]$} & 256 & & & & & & \\
\hline a45-m & {$[15,10,10]$} & 242 & & & & & & \\
\hline a45/FEF & & & & & & & {$[21,8,9]$} & 173 \\
\hline a46d & {$[13,18,12]$} & 87 & & & & & {$[15,14,14]$} & 266 \\
\hline$a 46 v$ & {$[17,14,13]$} & 190 & & & {$[17,13,12]$} & 343 & & \\
\hline a46v-1 & & & & & & & {$[17,13,11]$} & 333 \\
\hline a46v-m & & & & & & & {$[12,12,8]$} & 299 \\
\hline a46v (ps) & {$[12,14,10]$} & 94 & & & & & & \\
\hline F2 (PMd) & & & & & {$[15,6,17]$} & 279 & & \\
\hline F2-a (PMd) & {$[11,3,18]$} & 149 & & & & & & \\
\hline F2-1 (PMd) & {$[5,8,22]$} & 35 & & & & & & \\
\hline F2-m (PMd) & {$[10,5,15]$} & 205 & & & & & & \\
\hline
\end{tabular}




\begin{tabular}{|c|c|c|c|c|c|c|c|c|}
\hline \multirow{3}{*}{ ROI } & \multicolumn{4}{|c|}{ dPul-a } & \multicolumn{4}{|c|}{ dPul-p } \\
\hline & \multicolumn{2}{|c|}{ Monkey C } & \multicolumn{2}{|c|}{ Monkey B } & \multicolumn{2}{|c|}{ Monkey C } & \multicolumn{2}{|c|}{ Monkey B } \\
\hline & Peak voxel & Nr. of voxels & Peak voxel & Nr. of voxels & Peak voxel & Nr. of voxels & Peak voxel & Nr. of voxels \\
\hline \multicolumn{9}{|l|}{ Right hemisphere } \\
\hline Frontal lobe & & & & & & & & \\
\hline F5 (PMv) & {$[14,4,12]$} & 215 & & & & & & \\
\hline FEF & {$[16,7,13]$} & 318 & {$[12,10,16]$} & 41 & & & {$[13,11,16]$} & 265 \\
\hline FEF-d & {$[15,6,17]$} & 226 & & & & & & \\
\hline FEF-1 & & & & & {$[17,7,12]$} & 343 & & \\
\hline FEF-m & {$[13,7,14]$} & 256 & & & {$[16,7,16]$} & 330 & & \\
\hline \multicolumn{9}{|l|}{ Parietal lobe } \\
\hline 5 (somatosensory area) & {$[8,-24,22]$} & 29 & & & & & & \\
\hline $7 \mathrm{op}$ & & & & & & & {$[21,-20,14]$} & 142 \\
\hline $\mathrm{LIPd} / \mathrm{v}$ & & & & & {$[7,-25,16]$} & 167 & & \\
\hline LIPd & {$[11,-24,19]$} & 90 & & & & & & \\
\hline LIPv & {$[7,-23,14]$} & 162 & {$[10,-23,13]$} & 221 & {$[9,-21,15]$} & 116 & {$[8,-25,15]$} & 149 \\
\hline LIP/LOP & {$[7,-26,15]$} & 56 & & & & & & \\
\hline LOP & & & & & {$[7,-29,17]$} & 171 & & \\
\hline MIP & {$[4,-27,15]$} & 61 & {$[6,-25,16]$} & 196 & & & {$[5,-26,15]$} & 147 \\
\hline PIP & & & {$[5,-32,12]$} & 185 & & & & \\
\hline PIP/PO/MIP & & & {$[8,-32,10]$} & 301 & & & & \\
\hline PO/MIP & & & {$[7,-34,12]$} & 244 & & & & \\
\hline PO/MIP-d & & & & & & & {$[5,-33,17]$} & 62 \\
\hline PO/MIP-v & & & & & & & {$[6,-34,13]$} & 313 \\
\hline VIP & {$[7,-20,12]$} & 225 & {$[5,-27,12]$} & 151 & {$[6,-21,13]$} & 73 & {$[5,-27,11]$} & 151 \\
\hline \multicolumn{9}{|l|}{ Temporal lobe } \\
\hline $7 \mathrm{a}$ & & & {$[19,-21,17]$} & 180 & & & & \\
\hline $7 \mathrm{a}-1$ & & & & & & & {$[19,-25,16]$} & 231 \\
\hline $7 \mathrm{a}-\mathrm{m}$ & & & & & & & {$[13,-31,19]$} & 243 \\
\hline FST & {$[16,-18,3]$} & 263 & & & {$[17,-18,3]$} & 265 & & \\
\hline FST-d & & & {$[19,-21,4]$} & 257 & & & {$[19,-24,6]$} & 277 \\
\hline FST-v & & & {$[24,-18,0]$} & 221 & & & {$[20,-20,2]$} & 339 \\
\hline $\mathrm{IPa}$ & {$[18,-11,-5]$} & 282 & & & {$[19,-12,-6]$} & 214 & {$[18,-2,-11]$} & 330 \\
\hline
\end{tabular}




\begin{tabular}{|c|c|c|c|c|}
\hline \multirow{3}{*}{ ROI } & \multicolumn{4}{|c|}{ dPul-a } \\
\hline & \multicolumn{2}{|c|}{ Monkey C } & \multicolumn{2}{|c|}{ Monkey B } \\
\hline & Peak voxel & Nr. of voxels & Peak voxel & Nr. of voxe \\
\hline \multicolumn{5}{|c|}{ Right hemisphere } \\
\hline \multicolumn{5}{|c|}{ Temporal lobe } \\
\hline \multicolumn{5}{|c|}{$\mathrm{IPa}-\mathrm{d}$} \\
\hline \multicolumn{5}{|l|}{ IPa-v } \\
\hline MST & {$[13,-20,8]$} & 229 & & \\
\hline \multicolumn{5}{|l|}{ MST-1 } \\
\hline \multicolumn{5}{|l|}{ MST-m } \\
\hline MT & {$[15,-23,7]$} & 236 & & \\
\hline MT-d & {$[13,-26,15]$} & 200 & & \\
\hline MT-1 & & & {$[21,-25,8]$} & 302 \\
\hline MT-m & & & {$[14,-27,10]$} & 133 \\
\hline \multicolumn{5}{|l|}{ MT-v } \\
\hline MT/MST & {$[11,-23,11]$} & 266 & {$[17,-23,7]$} & 277 \\
\hline $\mathrm{PGa}$ & {$[17,-2,-8]$} & 26 & & \\
\hline \multirow{2}{*}{\multicolumn{5}{|c|}{ TAa }} \\
\hline & & & & \\
\hline TAa-d & {$[25,-12,3]$} & 74 & & \\
\hline TAa-v & {$[26,-4,-5]$} & 86 & & \\
\hline TEa & {$[24,-7,-7]$} & 214 & & \\
\hline \multicolumn{5}{|l|}{ TEa-d } \\
\hline \multicolumn{5}{|l|}{ TEa-v } \\
\hline \multicolumn{5}{|l|}{$\mathrm{TEa} / \mathrm{m}$} \\
\hline \multicolumn{5}{|l|}{ TEm-d } \\
\hline \multicolumn{5}{|l|}{ TEm-v } \\
\hline \multicolumn{5}{|l|}{ TE/TEO } \\
\hline \multirow{2}{*}{\multicolumn{5}{|c|}{ TEO }} \\
\hline \multicolumn{4}{|l|}{ TEO-d } & \\
\hline TEO-1 & {$[25,-15,4]$} & 88 & & \\
\hline TEO-m & {$[21,-17,3]$} & 189 & & \\
\hline \multicolumn{5}{|l|}{ TG-1 (sts) } \\
\hline \multicolumn{5}{|c|}{ TG-m (sts) } \\
\hline TG/RT & & & & \\
\hline
\end{tabular}

dPul-p

\begin{tabular}{ccc}
\multicolumn{2}{c}{ Monkey C } & \multicolumn{2}{c}{ Monkey B } \\
Peak voxel & Nr. of voxels & Peak voxel $\quad$ Nr. of voxels
\end{tabular}

Right hemisphere

(21, $-12,-7]$

$[19,-7,-9] \quad 240$

$[16,-25,15] \quad 258$

$[22,-23,12] \quad 182$

$[12,-29,14] \quad 311$

MT $\quad[15,-23,7] \quad 236$

MT-d [13, -26, 15] 200

137

$[19,-27,14] \quad 233$

$[22,-25,9] \quad 315$

$[15,-27,9] \quad 174$

$[19,-21,5] \quad 343$

$[19,-8,-5] \quad 325$

$[26,-4,-9] \quad 81$

$\begin{array}{cccc}{[19,-3,-10]} & 245 & & \\ & & {[25,-12,-8]} & 312 \\ & {[23,-7,-11]} & 320 \\ & {[22,-2,-12]} & 328 \\ & {[29,-15,-2]} & 342 \\ & {[29,-12,-6]} & 305 \\ & {[25,-18,0]} & 338 \\ & & \\ & & {[22,-24,6]} & 279 \\ & & {[29,-18,1]} & 273 \\ & {[23,-16,-3]} & 257 \\ & & {[22,3,-11]} & 257 \\ & {[18,2,-13]} & 294 \\ & {[24,3,-5]} & 248\end{array}$




\begin{tabular}{|c|c|c|c|c|c|c|c|c|}
\hline \multirow{3}{*}{ ROI } & \multicolumn{4}{|c|}{$\mathrm{dPul}-\mathrm{a}$} & \multicolumn{4}{|c|}{ dPul-p } \\
\hline & \multicolumn{2}{|c|}{ Monkey C } & \multicolumn{2}{|c|}{ Monkey B } & \multicolumn{2}{|c|}{ Monkey C } & \multicolumn{2}{|c|}{ Monkey B } \\
\hline & Peak voxel & Nr. of voxels & Peak voxel & Nr. of voxels & Peak voxel & Nr. of voxels & Peak voxel & Nr. of voxels \\
\hline \multicolumn{9}{|c|}{ Right hemisphere } \\
\hline \multicolumn{9}{|c|}{ Temporal lobe } \\
\hline TPO & {$[20,-14,4]$} & 218 & & & & & & \\
\hline TPO-a & & & & & {$[20,-12,0]$} & 347 & & \\
\hline TPO-d & {$[18,-18,6]$} & 235 & & & & & {$[24,-13,-2]$} & 258 \\
\hline TPO-p & & & & & {$[19,-15,4]$} & 311 & & \\
\hline TPO-v & & & & & & & {$[23,-7,-7]$} & 269 \\
\hline Tpt & & & & & & & {$[24,-23,14]$} & 248 \\
\hline \multicolumn{9}{|c|}{ Cingulate cortex } \\
\hline 23a (PCC) & {$[1,-10,12]$} & 81 & & & & & & \\
\hline $23 b(\mathrm{PCC})$ & {$[2,-19,14]$} & 102 & & & & & & \\
\hline $23 \mathrm{a} / \mathrm{b}(\mathrm{PCC})$ & {$[2,-23,9]$} & 159 & & & & & & \\
\hline v23b (PCC) & & & {$[3,-24,3]$} & 221 & & & & \\
\hline \multicolumn{9}{|c|}{ Somatosensory cortex } \\
\hline $1-2$ & & & & & {$[5,-9,17]$} & 16 & {$[29,-9,4]$} & 116 \\
\hline $3 a / b$ & & & & & & & {$[28,-1,6]$} & 92 \\
\hline \multicolumn{9}{|l|}{ Visual cortex } \\
\hline V1 & & & & & {$[11,-38,4]$} & 54 & {$[14,-38,3]$} & 128 \\
\hline V1 (cas) & {$[8,-31,4]$} & 61 & & & & & & \\
\hline V1-p & {$[16,-36,13]$} & 45 & & & & & & \\
\hline $\mathrm{V} 1 / \mathrm{V} 2$ (cas) & {$[8,-22,1]$} & 202 & & & & & & \\
\hline $\mathrm{V} 2$ & {$[7,-29,1]$} & 53 & & & {$[19,-31,10]$} & 72 & {$[3,-38,9]$} & 76 \\
\hline $\mathrm{V} 2-\mathrm{V}$ & & & {$[12,-39,-6]$} & 110 & & & & \\
\hline V2 (apos) & & & & & & & {$[4,-32,4]$} & 237 \\
\hline V2 (cas) & & & {$[9,-23,-2]$} & 128 & & & {$[12,-35,2]$} & 64 \\
\hline V2 (lus) & {$[16,-32,8]$} & 152 & & & & & {$[9,-39,14]$} & 87 \\
\hline V2-d (lus) & {$[13,-35,12]$} & 224 & & & & & & \\
\hline V2-1 (lus) & & & {$[7,-37,13]$} & 142 & & & & \\
\hline V2-m (lus) & & & {$[2,-37,11]$} & 97 & & & & \\
\hline
\end{tabular}




\begin{tabular}{|c|c|c|c|c|c|c|c|c|}
\hline \multirow{3}{*}{ ROI } & \multicolumn{4}{|c|}{ dPul-a } & \multicolumn{4}{|c|}{ dPul-p } \\
\hline & \multicolumn{2}{|c|}{ Monkey C } & \multicolumn{2}{|c|}{ Monkey B } & \multicolumn{2}{|c|}{ Monkey C } & \multicolumn{2}{|c|}{ Monkey B } \\
\hline & Peak voxel & Nr. of voxels & Peak voxel & Nr. of voxels & Peak voxel & Nr. of voxels & Peak voxel & Nr. of voxels \\
\hline \multicolumn{9}{|c|}{ Right hemisphere } \\
\hline \multicolumn{9}{|c|}{ Visual cortex } \\
\hline V2 (ots) & & & {$[13,-35,-4]$} & 62 & & & & \\
\hline V2 (pos) & {$[3,-29,7]$} & 216 & {$[3,-38,4]$} & 37 & & & & \\
\hline V2-p (pos) & {$[7,-34,8]$} & 42 & & & & & & \\
\hline V2-v (pos) & {$[5,-29,4]$} & 169 & & & & & & \\
\hline V3 & & & & & {$[20,-27,8]$} & 102 & & \\
\hline V3A & {$[13,-28,12]$} & 238 & & & {$[17,-30,11]$} & 75 & {$[11,-32,9]$} & 71 \\
\hline V3d & {$[12,-32,12]$} & 145 & & & & & {$[10,-38,12]$} & 150 \\
\hline V3d-a & & & & & & & {$[15,-34,9]$} & 292 \\
\hline V3d-a (lus) & & & {$[15,-35,9]$} & 137 & & & & \\
\hline V3d-1 & & & & & & & {$[12,-37,10]$} & 198 \\
\hline V3d-m & & & & & & & {$[6,-37,13]$} & 97 \\
\hline V3d-p (lus) & & & {$[11,-37,11]$} & 137 & & & & \\
\hline $\mathrm{V} 3 \mathrm{v}$ & & & {$[12,-27,-3]$} & 67 & {$[26,-24,4]$} & 87 & & \\
\hline $\mathrm{V} 3 \mathrm{v} / \mathrm{V} 2$ & & & {$[20,-37,-1]$} & 124 & & & & \\
\hline $\mathrm{V} 3 \mathrm{v} / \mathrm{V} 4 \mathrm{v}$ & & & {$[15,-24,-6]$} & 68 & & & & \\
\hline V4 & & & & & {$[21,-27,12]$} & 125 & & \\
\hline V4-a & & & & & & & {$[29,-22,8]$} & 119 \\
\hline V4-d & & & & & & & {$[21,-30,15]$} & 230 \\
\hline V4-p & & & & & & & {$[26,-27,12]$} & 258 \\
\hline V4/DP & & & & & {$[15,-26,17]$} & 245 & & \\
\hline $\mathrm{V} 4 / \mathrm{V} 4 \mathrm{t}$ & & & & & & & {$[25,-24,12]$} & 337 \\
\hline V4t & & & {$[25,-24,11]$} & 317 & {$[24,-20,9]$} & 262 & & \\
\hline V4v & {$[18,-21,-4]$} & 235 & & & & & & \\
\hline V4v-1 (ots) & & & {$[21,-26,-3]$} & 119 & & & & \\
\hline V4v-m (ots) & & & {$[17,-29,-4]$} & 113 & & & & \\
\hline \multicolumn{9}{|c|}{ Other cortical areas } \\
\hline Id (insula) & & & & & & & {$[21,-1,-2]$} & 324 \\
\hline Id (insula)/G & & & & & & & {$[20,-1,4]$} & 124 \\
\hline Id/Ia (insula) & & & & & & & {$[20,3,-2]$} & 136 \\
\hline
\end{tabular}




\begin{tabular}{|c|c|c|c|c|c|c|c|c|}
\hline \multirow{3}{*}{ ROI } & \multicolumn{4}{|c|}{ dPul-a } & \multicolumn{4}{|c|}{ dPul-p } \\
\hline & \multicolumn{2}{|c|}{ Monkey C } & \multicolumn{2}{|c|}{ Monkey B } & \multicolumn{2}{|c|}{ Monkey C } & \multicolumn{2}{|c|}{ Monkey B } \\
\hline & Peak voxel & Nr. of voxels & Peak voxel & Nr. of voxels & Peak voxel & Nr. of voxels & Peak voxel & Nr. of voxels \\
\hline \multicolumn{9}{|l|}{ Right hemisphere } \\
\hline \multicolumn{9}{|l|}{ Other cortical areas } \\
\hline $\mathrm{Id} / \mathrm{Pi}$ (insula) & & & & & & & {$[20,-8,-2]$} & 223 \\
\hline insula & {$[19,1,-2]$} & 36 & {$[21,-6,0]$} & 187 & {$[20,-3,3]$} & 173 & & \\
\hline retrosplenial cortex & & & {$[2,-19,8]$} & 69 & & & & \\
\hline \multicolumn{9}{|l|}{ Subcortical regions } \\
\hline amygdala & & & & & {$[13,2,-10]$} & 90 & {$[14,-3,-10]$} & 299 \\
\hline bsc & {$[10,-13,1]$} & 114 & {$[9,-17,0]$} & 122 & & & {$[11,-17,1]$} & 122 \\
\hline caudate & {$[4,-4,8]$} & 101 & & & {$[4,3,9]$} & 85 & & \\
\hline cerebellum & & & & & {$[9,-29,-7]$} & 151 & {$[16,-32,-6]$} & 89 \\
\hline dPul & {$[5,-14,4]$} & 292 & {$[7,-16,4]$} & 343 & {$[6,-14,2]$} & 343 & {$[7,-16,4]$} & 338 \\
\hline hippocampus & & & & & & & {$[12,-8,-8]$} & 123 \\
\hline LGN (thalamus) & & & {$[11,-14,1]$} & 74 & & & & \\
\hline MD (thalamus) & & & & & & & {$[4,-12,5]$} & 135 \\
\hline MD-a (thalamus) & & & {$[2,-9,4]$} & 31 & & & & \\
\hline MD-p (thalamus) & & & {$[4,-10,6]$} & 73 & & & & \\
\hline pallidum & & & {$[7,-6,-3]$} & 94 & & & & \\
\hline SC & & & {$[4,-18,-1]$} & 292 & {$[3,-14,-1]$} & 284 & {$[5,-18,-1]$} & 300 \\
\hline SC-a & {$[4,-14,0]$} & 308 & & & & & & \\
\hline SC-p & {$[3,-18,-1]$} & 290 & & & & & & \\
\hline thalamus & & & {$[6,-12,5]$} & 254 & & & & \\
\hline thalamus-a & {$[2,-5,6]$} & 26 & & & & & & \\
\hline thalamus-p & {$[4,-11,6]$} & 139 & & & & & & \\
\hline vPul & {$[8,-13,-2]$} & 256 & & & {$[9,-14,-3]$} & 286 & & \\
\hline \multicolumn{9}{|l|}{ Left hemisphere } \\
\hline \multicolumn{9}{|l|}{ Frontal lobe } \\
\hline $12 \mathrm{~m}$ & & & & & & & {$[-18,17,5]$} & 152 \\
\hline 31 & {$[-1,-21,17]$} & 90 & & & & & & \\
\hline $\mathrm{a} 44 / \mathrm{a} 45$ & {$[-13,5,9]$} & 89 & & & & & & \\
\hline a45 & & & & & {$[-15,6,11]$} & 201 & & \\
\hline
\end{tabular}




\begin{tabular}{|c|c|c|c|c|c|c|c|}
\hline \multirow{3}{*}{ ROI } & \multicolumn{3}{|c|}{ dPul-a } & \multicolumn{4}{|c|}{ dPul-p } \\
\hline & \multicolumn{2}{|c|}{ Monkey C } & Monkey B & \multicolumn{2}{|c|}{ Monkey C } & \multicolumn{2}{|c|}{ Monkey B } \\
\hline & Peak voxel & Nr. of voxels & Peak voxel Nr. of voxels & Peak voxel & Nr. of voxels & Peak voxel & Nr. of voxels \\
\hline \multicolumn{8}{|c|}{ Left hemisphere } \\
\hline \multicolumn{8}{|c|}{ Frontal lobe } \\
\hline $\mathrm{F} 2(\mathrm{PMd})$ & & & & {$[-12,-1,13]$} & 154 & & \\
\hline F5 (PMv) & {$[-11,1,14]$} & 60 & & & & & \\
\hline FEF & {$[-14,3,16]$} & 84 & & & & & \\
\hline SMA & & & & {$[-4,-1,18]$} & 40 & & \\
\hline \multicolumn{8}{|c|}{ Parietal lobe } \\
\hline AIP & & & & {$[-20,-14,15]$} & 56 & & \\
\hline LIPd & & & & {$[-15,-16,16]$} & 37 & & \\
\hline LIPd-p & {$[-8,-24,21]$} & 103 & & & & & \\
\hline $\mathrm{LIPd} / \mathrm{v}$ & {$[-14,-18,16]$} & 100 & & & & & \\
\hline $\mathrm{LIPv}$ & & & & {$[-9,-21,16]$} & 220 & & \\
\hline LOP/V3A & {$[-14,-30,15]$} & 122 & & & & & \\
\hline LOP & & & & {$[-8,-28,19]$} & 131 & & \\
\hline $\mathrm{PO}$ & {$[-4,-31,16]$} & 102 & & & & & \\
\hline \multicolumn{8}{|c|}{ Temporal lobe } \\
\hline $7 \mathrm{a}$ & & & & {$[-16,-23,16]$} & 37 & & \\
\hline $7 a-a$ & {$[-11,-25,21]$} & 86 & & & & & \\
\hline $7 a-1$ & {$[-16,-22,20]$} & 57 & & & & & \\
\hline $7 a-p$ & {$[-9,-27,22]$} & 109 & & & & & \\
\hline FST & {$[-16,-19,5]$} & 108 & & {$[-18,-20,5]$} & 192 & & \\
\hline $\mathrm{FST} / \mathrm{IPa}$ & {$[-22,-18,-1]$} & 54 & & & & & \\
\hline MST & & & & {$[-16,-21,13]$} & 70 & & \\
\hline MT & & & & {$[-19,-22,8]$} & 90 & & \\
\hline MT-d & {$[-16,-24,13]$} & 126 & & & & & \\
\hline MT-v & {$[-18,-23,7]$} & 26 & & & & & \\
\hline TE/TEO & {$[-23,-16,4]$} & 42 & & & & & \\
\hline TEO & & & & {$[-20,-17,4]$} & 376 & {$[-25,-20,1]$} & 78 \\
\hline TPO-a & & & & {$[-22,-14,3]$} & 297 & & \\
\hline TPO-p & & & & {$[-23,-20,11]$} & 15 & & \\
\hline
\end{tabular}




\begin{tabular}{|c|c|c|c|c|c|c|c|c|}
\hline \multirow{3}{*}{ ROI } & \multicolumn{4}{|c|}{$\mathrm{dPul}-\mathrm{a}$} & \multicolumn{4}{|c|}{ dPul-p } \\
\hline & \multicolumn{2}{|c|}{ Monkey C } & \multicolumn{2}{|c|}{ Monkey B } & \multicolumn{2}{|c|}{ Monkey C } & \multicolumn{2}{|c|}{ Monkey B } \\
\hline & Peak voxel & Nr. of voxels & Peak voxel & Nr. of voxels & Peak voxel & Nr. of voxels & Peak voxel & Nr. of voxels \\
\hline \multicolumn{9}{|c|}{ Left hemisphere } \\
\hline \multicolumn{9}{|c|}{ Somatosensory cortex } \\
\hline $1-2$ & & & & & {$[-5,-9,16]$} & 10 & & \\
\hline \multicolumn{9}{|l|}{ Visual cortex } \\
\hline V1 & & & & & {$[-12,-38,3]$} & 48 & & \\
\hline V1-a & & & & & & & {$[-26,-34,5]$} & 314 \\
\hline V1-a (cas) & {$[-8,-31,4]$} & 35 & & & & & & \\
\hline V1-d & {$[-13,-36,17]$} & 89 & & & & & & \\
\hline V1-dm & {$[-14,-37,13]$} & 291 & & & & & & \\
\hline V1-p & & & & & & & {$[-23,-36,7]$} & 258 \\
\hline $\mathrm{V} 2$ & & & & & {$[-18,-32,9]$} & 314 & & \\
\hline V2 (ios) & & & {$[-26,-30,3]$} & 294 & & & & \\
\hline V2-a (ios) & & & & & & & {$[-26,-31,2]$} & 329 \\
\hline V2-p (ios) & & & & & & & {$[-24,-35,3]$} & 288 \\
\hline V2 (lus) & & & {$[-23,-31,9]$} & 110 & & & & \\
\hline V2-a (lus) & & & & & & & {$[-25,-30,8]$} & 244 \\
\hline V2-m (lus) & {$[-7,-34,18]$} & 76 & & & & & & \\
\hline V2-p (lus) & & & & & & & {$[-23,-33,10]$} & 250 \\
\hline V2-v (lus) & {$[-16,-32,10]$} & 173 & & & & & & \\
\hline V3A & & & & & {$[-20,-28,9]$} & 212 & & \\
\hline $\mathrm{V} 3 \mathrm{v}$ & {$[-22,-30,-3]$} & 79 & {$[-27,-25,2]$} & 279 & {$[-20,-25,3]$} & 159 & & \\
\hline$V 3 v-a$ & & & & & & & {$[-27,-28,-1]$} & 226 \\
\hline V3v-p & & & & & & & {$[-23,-35,-2]$} & 161 \\
\hline V4 & {$[-25,-24,10]$} & 120 & {$[-29,-23,5]$} & 183 & {$[-25,-25,6]$} & 119 & {$[-28,-25,6]$} & 155 \\
\hline V4/DP & & & & & {$[-18,-29,14]$} & 141 & & \\
\hline \multicolumn{9}{|c|}{ Subcortical regions } \\
\hline caudate & & & & & & & {$[-4,7,0]$} & 108 \\
\hline cerebellum-d & & & & & {$[-2,-30,2]$} & 93 & & \\
\hline cerebellum-v & & & & & {$[-12,-29,-7]$} & 92 & & \\
\hline putamen & {$[-13,-1,5]$} & 29 & & & & & & \\
\hline
\end{tabular}


Table 3.4 Regions of interest (ROIs) defined for high-current stimulation in LIPd with coordinates of ROI peak voxels ([x, y, z]) as distance from AC-PC origin in millimeters and total number of voxels per ROI.

\begin{tabular}{|c|c|c|c|c|c|c|c|c|}
\hline \multirow{3}{*}{ ROI } & \multicolumn{4}{|c|}{ LIPd-a } & \multicolumn{4}{|c|}{ LIPd-p } \\
\hline & \multicolumn{2}{|c|}{ Monkey C } & \multicolumn{2}{|c|}{ Monkey B } & \multicolumn{2}{|c|}{ Monkey C } & \multicolumn{2}{|c|}{ Monkey B } \\
\hline & Peak voxel & Nr. of voxels & Peak voxel & Nr. of voxels & Peak voxel & Nr. of voxels & Peak voxel & Nr. of voxels \\
\hline \multicolumn{9}{|l|}{ Right hemisphere } \\
\hline Frontal lobe & & & & & & & & \\
\hline $8 \mathrm{Bs}$ & {$[9,9,13]$} & 337 & & & & & & \\
\hline 8Bs-a & & & & & {$[10,11,16]$} & 235 & & \\
\hline 8Bs-p & & & & & {$[9,9,13]$} & 337 & & \\
\hline $8 \mathrm{Bs} / \mathrm{FEF}$ & & & {$[11,6,13]$} & 315 & & & & \\
\hline 111 & & & {$[14,22,7]$} & 251 & & & & \\
\hline a44/a45 & {$[11,5,11]$} & 307 & & & & & & \\
\hline a46d-a & & & {$[16,12,13]$} & 191 & & & & \\
\hline a46d-p & & & {$[17,8,14]$} & 276 & & & & \\
\hline $\mathrm{a} 46 \mathrm{~d} / \mathrm{v}$ & {$[14,10,14]$} & 328 & & & & & & \\
\hline a46v & {$[11,15,10]$} & 309 & {$[13,14,9]$} & 310 & {$[12,12,10]$} & 209 & & \\
\hline F2 (PMd) & & & {$[18,1,17]$} & 157 & & & & \\
\hline F4 (PMv) & & & & & & & {$[27,2,3]$} & 77 \\
\hline FEF & & & & & & & {$[12,5,12]$} & 156 \\
\hline FEF-a & & & {$[14,12,16]$} & 171 & & & & \\
\hline FEF-1 & {$[14,9,17]$} & 246 & & & & & & \\
\hline FEF-m & {$[13,6,15]$} & 337 & & & & & & \\
\hline FEF-p & & & {$[14,8,16]$} & 332 & & & & \\
\hline \multicolumn{9}{|l|}{ Parietal lobe } \\
\hline 5 (somatosensory area) & & & & & & & {$[2,-30,21]$} & 83 \\
\hline 5 (somatosensory area, cis) & & & & & {$[5,-23,22]$} & 147 & & \\
\hline 5 (somatosensory area, ips) & & & & & {$[10,-16,16]$} & 176 & & \\
\hline $7 \mathrm{~m}$ & & & & & {$[2,-26,10]$} & 281 & & \\
\hline 7m (apos) & {$[3,-27,6]$} & 295 & & & & & & \\
\hline $7 \mathrm{~m} / \mathrm{PO}$ & {$[1,-28,16]$} & 164 & & & & & & \\
\hline LIPd & & & {$[11,-25,18]$} & 335 & & & {$[7,-30,19]$} & 324 \\
\hline LIPd-a & {$[10,-20,16]$} & 323 & {$[15,-22,16]$} & 341 & & & {$[10,-24,15]$} & 274 \\
\hline
\end{tabular}




\begin{tabular}{|c|c|c|c|c|c|c|c|c|}
\hline \multirow{3}{*}{ ROI } & \multicolumn{4}{|c|}{ LIPd-a } & \multicolumn{4}{|c|}{ LIPd-p } \\
\hline & \multicolumn{2}{|c|}{ Monkey C } & \multicolumn{2}{|c|}{ Monkey B } & \multicolumn{2}{|c|}{ Monkey C } & \multicolumn{2}{|c|}{ Monkey B } \\
\hline & Peak voxel & Nr. of voxels & Peak voxel & Nr. of voxels & Peak voxel & Nr. of voxels & Peak voxel & Nr. of voxels \\
\hline \multicolumn{9}{|c|}{ Right hemisphere } \\
\hline \multicolumn{9}{|c|}{ Parietal lobe } \\
\hline LIPd-p & {$[6,-26,15]$} & 317 & {$[7,-29,18]$} & 343 & {$[6,-26,15]$} & 317 & {$[7,-26,15]$} & 343 \\
\hline $\mathrm{LIPd} / \mathrm{v}$ & & & & & {$[12,-18,15]$} & 177 & & \\
\hline LIPv & & & {$[8,-24,14]$} & 343 & & & & \\
\hline LOP & {$[7,-29,16]$} & 343 & {$[9,-33,13]$} & 310 & {$[7,-29,15]$} & 343 & {$[9,-34,15]$} & 288 \\
\hline MIP & & & {$[4,-29,15]$} & 203 & & & {$[5,-28,15]$} & 245 \\
\hline MIP/PIP & & & & & {$[6,-28,12]$} & 337 & & \\
\hline $\mathrm{PO}$ & {$[2,-31,16]$} & 237 & {$[4,-35,18]$} & 245 & & & {$[5,-34,16]$} & 177 \\
\hline PO-1 & & & & & {$[6,-31,14]$} & 343 & & \\
\hline PO-m & & & & & {$[2,-31,10]$} & 324 & & \\
\hline VIP & {$[6,-20,11]$} & 280 & & & {$[6,-22,12]$} & 282 & {$[7,-26,11]$} & 163 \\
\hline \multicolumn{9}{|c|}{ Temporal lobe } \\
\hline $7 \mathrm{a}$ & {$[12,-26,18]$} & 300 & & & {$[12,-25,17]$} & 342 & {$[12,-28,19]$} & 343 \\
\hline $7 a-a$ & & & {$[15,-26,18]$} & 333 & & & & \\
\hline $7 a-p$ & & & {$[13,-30,19]$} & 323 & & & & \\
\hline FST & {$[16,-17,3]$} & 343 & & & {$[16,-17,3]$} & 343 & & \\
\hline FST-d & & & {$[18,-24,6]$} & 85 & & & {$[20,-20,2]$} & 252 \\
\hline FST-v & & & {$[20,-19,2]$} & 267 & & & {$[17,-24,6]$} & 172 \\
\hline $\mathrm{IPa}$ & & & & & & & {$[21,-12,-7]$} & 212 \\
\hline IPa-a & & & {$[21,-10,-8]$} & 308 & & & & \\
\hline IPa-p & & & {$[23,-14,-7]$} & 287 & & & & \\
\hline MST & & & & & & & {$[13,-27,17]$} & 317 \\
\hline MST-a & & & {$[15,-25,14]$} & 309 & & & & \\
\hline MST-d & {$[12,-23,14]$} & 343 & & & {$[12,-23,14]$} & 343 & & \\
\hline MST-p & & & {$[13,-29,15]$} & 343 & & & & \\
\hline MST-v & {$[13,-20,7]$} & 322 & & & {$[14,-22,12]$} & 322 & & \\
\hline MT & {$[16,-24,8]$} & 249 & & & {$[14,-25,11]$} & 208 & {$[14,-28,13]$} & 284 \\
\hline PGa & {$[18,-10,-4]$} & 325 & & & {$[18,-9,-5]$} & 336 & & \\
\hline TEm & & & & & & & {$[28,-12,-5]$} & 277 \\
\hline TEO (pmts) & & & {$[29,-20,-4]$} & 283 & & & & \\
\hline
\end{tabular}




\begin{tabular}{l}
\hline \\
ROI \\
\hline Right hemisphe \\
Temporal lobe \\
TEO-1 \\
TEO-v \\
TEpd \\
TPO \\
TPO-a \\
TPO-1 \\
TPO-m \\
Cingulate cortex \\
23b (PCC) \\
23c (PCC) \\
PCC (cis) \\
v23b (PCC) \\
v23b (PCC)/V2 \\
Somatosensory cort \\
1-2 \\
SII \\
Visual cortex \\
V1 \\
V1-dm \\
V1-1 \\
V1-1 (cas) \\
V1-m \\
V1-m (cas) \\
V1-p \\
V1-p (cas) \\
V1-v \\
V1/V2 \\
\end{tabular}

\section{LIPd-a}

Monkey

LIPd-p

Monkey C

Monkey B

Peak voxel Nr. of voxels Peak voxel Nr. of voxels Peak voxel Nr. of voxels Peak voxel Nr. of voxels

hemisphere

[30,

$[30,-15,-2] \quad 159$

$[26,-21,-8]$

149

$[26,-5,-8]$

114

$[22,-12,2] \quad 287$

$[20,-15,4] \quad 292$

$[18,-19,7]$

292

$[20,-15,4]$

292

$[2,-18,14] \quad 168$

$[5,-18,17] \quad 39$

$[2,-24,9] \quad 263$

$[2,-25,10]$

$[2,-21,9]$

$[7,-26,0]$

108

$\begin{array}{ll}{[1,-14,13]} & 176 \\ {[6,-18,18]} & 160\end{array}$

285

$[21,-11,8] \quad 14$

$[2,-14,17]$

84

$[21,-8,6]$

155

$[13,-33,3]$

134

$\begin{array}{cc}{[11,-40,5]} & 130 \\ {[3,-43,8]} & 259 \\ {[25,-39,2]} & 185 \\ {[6,-42,3]} & 129 \\ {[7,-44,6]} & 245 \\ {[12,-29,1]} & 79\end{array}$

$[12,-36,15]$

337

$[16,-34,15]$

274

$[7,-38,15] \quad 275$

$[16,-35,3] \quad 166$

$[9,-36,1] \quad 94$

$[15,-38,1] \quad 122$

$[1,-35,11] \quad 267$

$[13,-40,4]$

218 


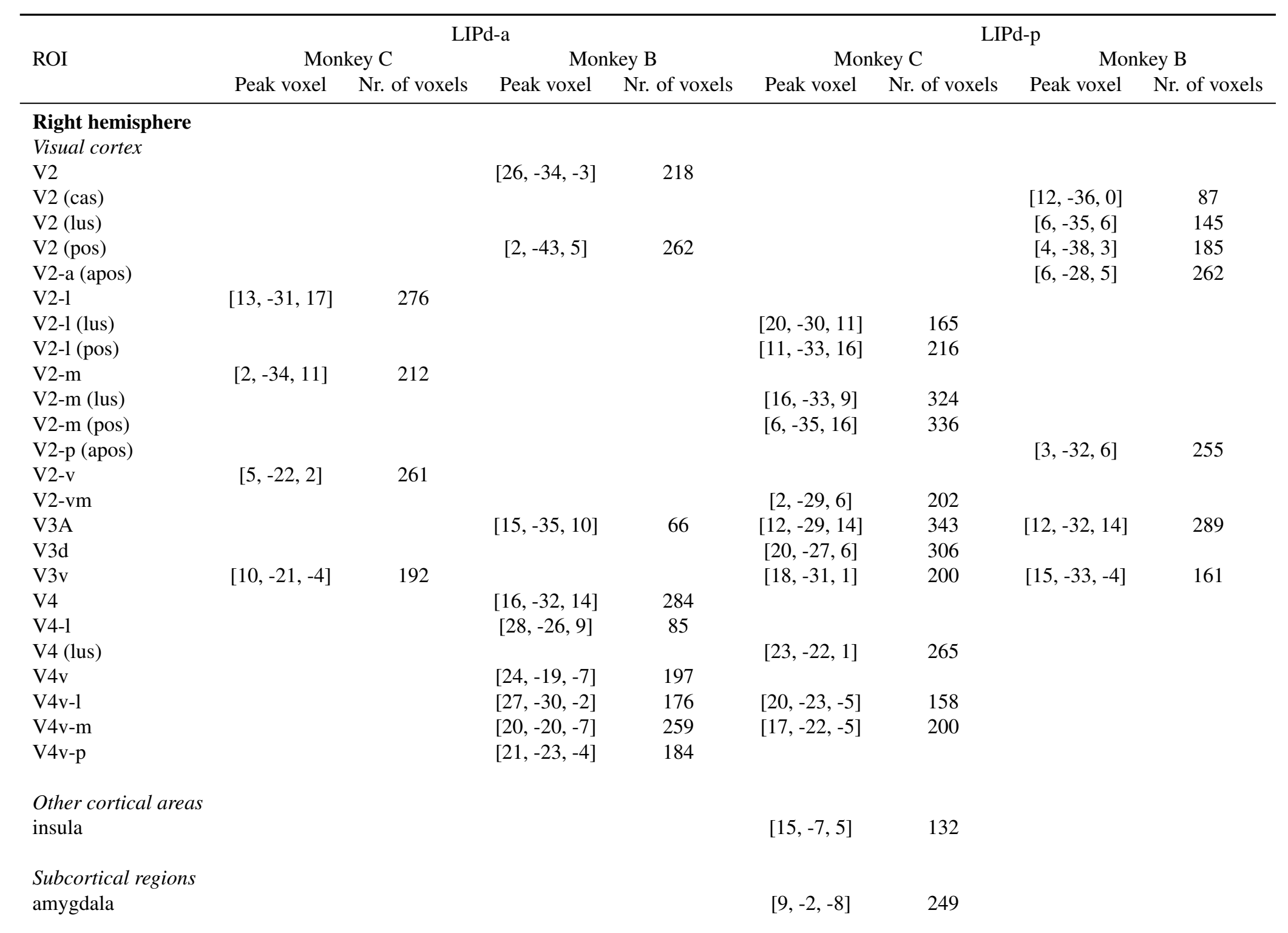




\begin{tabular}{|c|c|c|c|c|c|c|c|c|}
\hline \multirow{3}{*}{ ROI } & \multicolumn{4}{|c|}{ LIPd-a } & \multicolumn{4}{|c|}{ LIPd-p } \\
\hline & \multicolumn{2}{|c|}{ Monkey C } & \multicolumn{2}{|c|}{ Monkey B } & \multicolumn{2}{|c|}{ Monkey C } & \multicolumn{2}{|c|}{ Monkey B } \\
\hline & Peak voxel & Nr. of voxels & Peak voxel & Nr. of voxels & Peak voxel & Nr. of voxels & Peak voxel & Nr. of voxels \\
\hline \multicolumn{9}{|l|}{ Right hemisphere } \\
\hline Subcortical regions & & & & & & & & \\
\hline brainstem & & & & & & & {$[6,-18,-11]$} & 116 \\
\hline caudate & {$[5,-3,8]$} & 129 & & & {$[6,-4,9]$} & 100 & & \\
\hline cerebellum & & & {$[11,-35,-6]$} & 37 & {$[7,-27,-3]$} & 253 & {$[11,-32,-5]$} & 84 \\
\hline cerebellum-a & {$[6,-21,-7]$} & 37 & & & & & & \\
\hline cerebellum-d & {$[5,-32,-1]$} & 62 & {$[4,-38,-4]$} & 97 & & & & \\
\hline cerebellum-1 & & & {$[15,-32,-6]$} & 72 & & & & \\
\hline cerebellum-p & {$[9,-28,-8]$} & 24 & {$[9,-41,-8]$} & 108 & & & & \\
\hline $\mathrm{dPul}$ & & & & & {$[8,-16,3]$} & 143 & & \\
\hline hippocampus & {$[13,-14,-8]$} & 60 & {$[15,-12,-10]$} & 167 & {$[15,-11,-8]$} & 141 & & \\
\hline nucleus accumbens & & & {$[4,6,-1]$} & 104 & & & & \\
\hline putamen & {$[13,-4,4]$} & 62 & & & {$[12,1,5]$} & 45 & & \\
\hline STN/thalamus & {$[5,-10,-2]$} & 38 & & & & & & \\
\hline $\mathrm{vPul}$ & & & & & {$[10,-14,-2]$} & 266 & & \\
\hline \multicolumn{9}{|l|}{ Left hemisphere } \\
\hline Frontal lobe & & & & & & & & \\
\hline $12 \mathrm{~m}$ & & & {$[-16,17,5]$} & 94 & & & & \\
\hline $\mathrm{a} 46 \mathrm{~d} / \mathrm{v}$ & {$[-12,7,13]$} & 51 & & & & & & \\
\hline F1 (M1, cis) & & & & & {$[-3,-6,18]$} & 34 & & \\
\hline F1 (M1, cs) & & & & & {$[-9,-9,20]$} & 88 & & \\
\hline $\mathrm{F} 1 / 3 \mathrm{a} / \mathrm{b}$ & & & & & {$[-15,-5,12]$} & 143 & & \\
\hline F2-1 (PMd) & & & {$[-19,-3,19]$} & 90 & & & & \\
\hline F2-m (PMd) & & & {$[-13,-2,20]$} & 77 & & & & \\
\hline F5 (PMv) & & & {$[-20,2,10]$} & 81 & & & & \\
\hline \multicolumn{9}{|l|}{ Parietal lobe } \\
\hline 5 (somatosensory area, cis) & & & & & {$[-4,-21,22]$} & 122 & & \\
\hline 5-1 (somatosensory area, ips) & & & & & {$[-14,-14,17]$} & 63 & & \\
\hline 5-m (somatosensory area, ips) & & & & & {$[-9,-17,15]$} & 160 & & \\
\hline 7 op-d & & & & & {$[-20,-17,14]$} & 105 & & \\
\hline
\end{tabular}




\begin{tabular}{|c|c|c|c|c|c|c|c|c|}
\hline \multirow{3}{*}{ ROI } & \multicolumn{4}{|c|}{ LIPd-a } & \multicolumn{4}{|c|}{ LIPd-p } \\
\hline & \multicolumn{2}{|c|}{ Monkey C } & \multicolumn{2}{|c|}{ Monkey B } & \multicolumn{2}{|c|}{ Monkey C } & \multicolumn{2}{|c|}{ Monkey B } \\
\hline & Peak voxel & Nr. of voxels & Peak voxel & Nr. of voxels & Peak voxel & Nr. of voxels & Peak voxel & Nr. of voxels \\
\hline \multicolumn{9}{|c|}{ Left hemisphere } \\
\hline \multicolumn{9}{|c|}{ Parietal lobe } \\
\hline $7 \mathrm{op}-\mathrm{v}$ & & & & & {$[-18,-12,10]$} & 241 & & \\
\hline AIP & & & & & {$[-21,-8,13]$} & 89 & & \\
\hline LIPd & & & {$[-12,-24,20]$} & 269 & {$[-7,-23,19]$} & 226 & & \\
\hline LIPd-a & {$[-13,-19,16]$} & 111 & & & & & {$[-11,-26,20]$} & 222 \\
\hline LIPd-p & {$[-7,-23,19]$} & 226 & & & & & {$[-9,-28,18]$} & 67 \\
\hline $\mathrm{LIPd} / \mathrm{v}$ & {$[-7,-22,16]$} & 248 & & & & & & \\
\hline $\mathrm{LIP} / 7 \mathrm{a}$ & {$[-10,-23,21]$} & 321 & & & & & & \\
\hline LOP & {$[-7,-28,20]$} & 223 & & & {$[-7,-30,19]$} & 250 & & \\
\hline $\mathrm{PO}$ & & & & & {$[-3,-32,10]$} & 218 & & \\
\hline VIP & & & & & {$[-7,-17,12]$} & 192 & & \\
\hline \multicolumn{9}{|c|}{ Temporal lobe } \\
\hline $7 \mathrm{a}$ & & & {$[-13,-29,18]$} & 115 & & & & \\
\hline MST & {$[-12,-23,15]$} & 8 & & & & & {$[-16,-25,14]$} & 216 \\
\hline MST-d & & & & & {$[-12,-22,13]$} & 136 & & \\
\hline MST-v & & & & & {$[-11,-19,10]$} & 89 & & \\
\hline $\mathrm{PGa}$ & & & & & {$[-18,-13,1]$} & 202 & & \\
\hline $\mathrm{PGa} / \mathrm{TPO}$ & {$[-20,-12,2]$} & 219 & & & & & & \\
\hline $\mathrm{TEa}$ & & & {$[-19,-1,-13]$} & 107 & & & & \\
\hline TEO & {$[-28,-17,1]$} & 101 & & & & & & \\
\hline TEO (pmts) & & & {$[-28,-21,-1]$} & 94 & & & & \\
\hline ТPO & & & & & {$[-19,-16,6]$} & 257 & & \\
\hline \multicolumn{9}{|c|}{ Cingulate cortex } \\
\hline $23 b(\mathrm{PCC})$ & & & & & {$[-2,-17,12]$} & 183 & & \\
\hline $24 \mathrm{a}(\mathrm{ACC})$ & & & {$[-1,-5,12]$} & 108 & & & & \\
\hline $24 \mathrm{c}(\mathrm{ACC})$ & & & & & {$[-4,-1,15]$} & 101 & & \\
\hline $\mathrm{PCC}$ & {$[-2,-18,16]$} & 64 & & & & & & \\
\hline v23b (PCC) & & & {$[-2,-25,11]$} & 212 & & & {$[-1,-21,8]$} & 91 \\
\hline
\end{tabular}




\begin{tabular}{|c|c|c|c|c|c|c|c|c|}
\hline \multirow{3}{*}{ ROI } & \multicolumn{4}{|c|}{ LIPd-a } & \multicolumn{4}{|c|}{ LIPd-p } \\
\hline & \multicolumn{2}{|c|}{ Monkey C } & \multicolumn{2}{|c|}{ Monkey B } & \multicolumn{2}{|c|}{ Monkey C } & \multicolumn{2}{|c|}{ Monkey B } \\
\hline & Peak voxel & Nr. of voxels & Peak voxel & Nr. of voxels & Peak voxel & Nr. of voxels & Peak voxel & Nr. of voxels \\
\hline \multicolumn{9}{|l|}{ Left hemisphere } \\
\hline \multicolumn{9}{|l|}{ Somatosensory cortex } \\
\hline $3 \mathrm{a} / \mathrm{b}$ & & & & & {$[-10,-13,22]$} & 127 & {$[-27,-1,5]$} & 54 \\
\hline SII & {$[-21,-10,8]$} & 114 & & & & & & \\
\hline \multicolumn{9}{|l|}{ Visual cortex } \\
\hline V1 & & & {$[-5,-45,6]$} & 205 & {$[-15,-35,16]$} & 295 & {$[-16,-32,4]$} & 134 \\
\hline V2 & {$[-6,-29,4]$} & 46 & {$[-23,-33,9]$} & 162 & {$[-21,-25,4]$} & 148 & {$[-13,-31,4]$} & 140 \\
\hline $\mathrm{V} 2 / \mathrm{V} 3 \mathrm{v}$ & & & {$[-23,-29,3]$} & 165 & & & & \\
\hline V3A & & & & & {$[-18,-28,10]$} & 135 & & \\
\hline V3d & & & {$[-15,-35,11]$} & 118 & {$[-15,-31,11]$} & 190 & & \\
\hline V4 & & & {$[-25,-28,8]$} & 282 & & & & \\
\hline V4-1 & & & {$[-28,-23,3]$} & 177 & & & & \\
\hline $\mathrm{V} 4 \mathrm{v}$ & & & & & {$[-16,-24,-4]$} & 275 & & \\
\hline \multicolumn{9}{|l|}{ Other cortical areas } \\
\hline AI & {$[-19,-12,5]$} & 115 & & & & & & \\
\hline insula & {$[-16,-9,7]$} & 44 & & & {$[-19,-7,2]$} & 173 & & \\
\hline retrosplenial cortex & & & & & {$[-1,-7,12]$} & 153 & & \\
\hline \multicolumn{9}{|l|}{ Subcortical regions } \\
\hline amygdala & & & & & {$[-12,-2,-8]$} & 153 & & \\
\hline caudate & & & & & {$[-2,-2,9]$} & 74 & & \\
\hline cerebellum & & & & & {$[-8,-28,-4]$} & 207 & & \\
\hline $\mathrm{dPul}$ & {$[-7,-15,3]$} & 17 & & & {$[-8,-15,4]$} & 40 & & \\
\hline hippocampus-1 & & & {$[-15,-8,-11]$} & 267 & & & & \\
\hline hippocampus-m & & & {$[-10,-7,-10]$} & 137 & & & & \\
\hline hypothalamic nucleus & & & & & & & & \\
\hline putamen & {$[-14,-2,4]$} & 57 & & & & & & \\
\hline thalamus & {$[-8,-12,5]$} & 45 & & & {$[-7,-11,4]$} & 80 & & \\
\hline vPul & & & & & {$[-11,-14,1]$} & 125 & & \\
\hline
\end{tabular}

Somatosensory cortex

$3 \mathrm{a} / \mathrm{b}$

$[-21,-10,8$

$[-15,-35,16]$

295

$\mathrm{V} 2 / \mathrm{V} 3 \mathrm{v}$

V3d

$[-19,-12,5]$

$[-16,-9,7]$

$[-11,-14,1]$ 


\subsection{Discussion}

Electrical microstimulation of $\mathrm{dPul}$ consistently activated a cortical functional network comprising dorsolateral and ventrolateral prefrontal cortex (vlPFC), dorsal and ventral premotor cortex, the dorsal and the ventral bank and the fundus of STS as well as PPC, PCC, insular cortex, somatosensory cortex, and primary and extrastriate visual cortex. However, dPul was also found to have consistent functional connections with subcortical structures such as amygdala, cerebellum, and other thalamic nuclei. A similar cortical effective connectivity pattern was found for LIPd with consistent activation in dorsolateral prefrontal cortex (dlPFC), the dorsal bank and the fundus of STS, widespread activity in IPS/PPC, and additional functional connections with PCC, somatosensory cortex, and primary and extrastriate visual cortex. The great similarity between the effective connectivity patterns of $\mathrm{dPul}$ and LIPd provides evidence that dPul plays a functional role in the brain network involved in visuospatial attention and visuomotor processing (Corbetta, 1998; Kagan et al., 2010). Importantly, dPul stimulation-induced BOLD activation was not restricted to the stimulated hemisphere but unilateral $\mathrm{dPul}$ stimulation also activated various brain regions in the opposite hemisphere. Since, to our knowledge, monosynaptic anatomical connections between pulvinar and regions in the opposite hemisphere have not been reported in the literature, our findings strongly indicate polysynaptic transmission of the stimulation-induced neuronal signals. Moreover, electrical microstimulation of dPul and LIPd changed space representations within the activated brain network in a task-dependent manner and the strength of microstimulation effects was further influenced by the spatial tuning of the activated brain regions.

\subsubsection{Effective connectivity of dPul and LIP}

The diverse effective connectivity pattern of dPul nicely corresponds to its extensive anatomical connections found in various histological studies. More specifically, using the combination of fMRI and electrical microstimulation we replicated findings of anatomical studies showing that $\mathrm{dPul}$ has connections with cortical areas in dorsolateral and vlPFC (Asanuma et al., 1985; Barbas et al., 1991; Bos \& Benevento, 1975; Contini et al., 2010; Romanski et al., 1997), the dorsal (Burton \& Jones, 1976; Yeterian \& Pandya, 1989, 1991) and the ventral bank (Ungerleider et al., 1984; Webster et al., 1993; Yeterian \& Pandya, 1989, 1991) and the fundus of STS (Shipp, 2001). Moreover, in accordance with previous anatomical studies we found functional connections of dPul with PPC (Blatt et al., 1990; Hardy \& Lynch, 1992; Romanski et al., 1997), PCC (Baleydier \& Mauguiere, 1987; Buckwalter et al., 2008; Shibata \& Yukie, 2003), insular cortex (Friedman \& Murray, 1986; Mufson \& Mesulam, 1984), and 
extrastriate visual cortex (Shipp, 2001; Ungerleider et al., 2014; Yeterian \& Pandya, 1997). dPul stimulation-induced BOLD activity was also found in premotor cortex which may be driven by direct projections from pulvinar to both PMd and PMv (Cappe et al., 2009; Morel et al., 2005). As mentioned above, stimulation-induced BOLD activation may also be propagated polysynaptically (Matsui et al., 2012). Therefore, premotor activity in our study might further be driven indirectly via dPul stimulation-induced activation in PPC (Tanne-Gariepy et al., 2002), STS (Luppino et al., 2001), SC (Fries, 1984), and via monosynaptic and multisynaptic projections from dorsolateral and vlPFC, respectively (Luppino et al., 2003; Takahara et al., 2012). Although direct anatomical connections of dPul and somatosensory cortex have rarely been reported (Acuna et al., 1990), we also found consistent effective connectivity of dPul with somatosensory cortex. Pulvinar connections with somatosensory cortex were most consistently found for PA (Acuna et al., 1990; Darian-Smith \& Darian-Smith, 1993; Grieve et al., 2000; Jones et al., 1979; Pons \& Kaas, 1985), which lies anterior and adjacent to PM (Calabrese et al., 2015). Hence, the activation in somatosensory cortex resulting from dPul stimulation might be caused by coactivation of PA. This explanation, however, seems unlikely because we found consistent and even stronger activation in somatosensory cortex when stimulating the posterior part of dPul compared to anterior dPul sites. A more likely explanation might be that the dPul stimulation-induced BOLD activity in somatosensory cortex was caused by polysynaptic propagation of BOLD activation. Although projections from SC to dPul have rarely been reported (Benevento \& Fallon, 1975), dPul stimulation consistently coactivated SC. Moreover, electrical microstimulation of SC was shown to enhance BOLD signal in the somatosensory cortex (Field et al., 2008). Hence, the enhanced BOLD activity observed in somatosensory cortex in our study might result from polysynaptic activation through SC and, additionally, prefrontal cortex (Yeterian et al., 2012). Similarly, $\mathrm{dPul}$ stimulation-induced activation in primary visual cortex might result from coactivation of SC as collicular projections from primary visual cortex have been reported previously (Fries, 1984) and SC microstimulation leads to BOLD activation in V1 (Field et al., 2008). Moreover, BOLD activation in primary visual cortex might also be the result of polysynaptic transmission of dPul stimulation-induced activation in extrastriate visual areas (Felleman \& Van Essen, 1991) including area MT (Lewis \& Van Essen, 2000). In one animal dPul stimulation additionally activated areas in orbital prefrontal cortex which is in line with histological studies identifying anatomical connections between the two regions (Morecraft et al., 1992; Romanski et al., 1997; Trojanowski \& Jacobson, 1976).

Low-current dPul stimulation in one animal confirmed dorsolateral (FEF) and vlPFC (a45), premotor cortex (PMv), the ventral bank (MT) and the fundus of STS (IPa, FST), and extrastriate visual cortex (V3) as the main hubs of the pulvino-cortical network underlining 
the pulvinar's role in visuomotor behavior and attention (Desimone et al., 1990; Petersen et al., 1987; Ward \& Danziger, 2005; Wilke et al., 2010). Moreover, in accordance with histological studies reporting reciprocal projections between dPul and amygdala (Jones \& Burton, 1976; Price \& Amaral, 1981), amygdala was found to be consistently activated during dPul stimulation supporting the involvement of the pulvinar in the processing of emotional visual stimuli (Hakamata et al., 2016; Le et al., 2016; Van Le et al., 2013; Ward et al., 2005). Low-current stimulation in monkey $\mathrm{C}$ also allowed for the direct comparison of the effective connectivity patterns of dPul and vPul. vPul stimulation also activated dorsolateral (FEF) and vlPFC (a45), the ventral bank (TEO) and the fundus of STS and led to extensive activation in primary and extrastriate visual cortex. Histological studies have also identified robust vPul anatomical connections with visual cortex (Adams et al., 2000; Rockland et al., 1999; Shipp, 2001) and STS (Benevento \& Rezak, 1976; Shipp, 2001; Yeterian \& Pandya, 1991). However, projections from vPul to prefrontal cortex have not been reported. Thus, vPul stimulation might have activated prefrontal areas indirectly through multiple synapses. Since vPul stimulation led to strong activation in SC it seems likely that FEF was activated via SC-FEF connections (Sommer \& Wurtz, 1998) while vPul stimulation-induced activity in vlPFC may be explained by polysynaptic signal transmission via the fundus of STS (Yeterian et al., 2012). Again, the finding that even unilateral low-current stimulation of both dPul and vPul led to BOLD activation in the opposite hemisphere strongly supports the hypothesis that microstimulation-induced BOLD signals cannot only be propagated monosynaptically but also via multiple synapses.

LIPd stimulation activated a brain network comprising dlPFC, the dorsal bank and the fundus of STS, and various areas on the lateral and medial bank of IPS including the medial intraparietal area (MIP) and somatosensory area 5. Stimulation of LIPd also induced BOLD activation in PCC and striate and extrastriate visual cortex. Comparing the effective connectivity patterns of dPul and LIPd revealed dIPFC (FEF, a46), the dorsal bank (TPO, MST) and the fundus of STS (FST), the lateral bank (LIPd, LIPv), the fundus (VIP) and the medial bank of IPS (MIP), PCC, and striate and extrastriate visual cortex (V1, V2, V3, V4) as the common nodes of the functional networks of dPul and LIP. The similarity between the effective connectivity of dPul and LIP suggests dPul as an important hub in the functional brain network involved in visuomotor processing and execution (Kagan et al., 2010). This finding is further supported by previous studies showing a reduction in BOLD signal in a very similar brain network following pharmacological inactivation of both dPul (Wilke et al., sion) and LIP (Wilke et al., 2012). Interestingly, the common network of dPul and LIPd includes MIP, an area that plays an important role in eye-hand coordination (Hwang et al., 2014) and spatial decision making related to arm movements (Christopoulos et al., 2015; 
Kubanek et al., 2015) and that receives thalamocortical inputs from the dorsal and lateral portions of PL (Prevosto et al., 2009). These findings suggest that dPul might be part of a thalamocortical pathway involved in the coordination of eye and arm movements.

The general effective connectivity pattern of LIPd in our study matches with the LIP effective connectivity found recently with activation in IPS, the fundus of STS, FEF, and extrastriate visual cortex (Premereur et al., 2015b). In our study, however, we additionally compared the connectivity of a more anterior site in LIPd to that of a more posterior LIPd site and observed an interesting difference between the two sites: BOLD activation in dorsolateral prefrontal cortex was more extensive when stimulating an anterior site in LIPd compared to a more posterior LIPd location. Differences in anterior and posterior effective connectivity patterns have already been found in the anterior intraparietal area, which is located on the lateral bank of IPS, anterior and adjacent to area LIP (Premereur et al., 2015b). These findings suggest that the current parcellation of PPC areas, particularly area LIP, may not be sufficient to account for their functional heterogeneity (Premereur et al., 2011). According to our data, a segregation of LIPd into an anterior and a posterior portion may help to further describe functional and connectional properties of area LIP, as supported by neuroimaging studies on the topographic organizatio of LIP (Patel et al., 2014).

\subsubsection{Task-dependent effects of microstimulation of dPul and LIP on BOLD activity}

The magnitude of the effects of microstimulation of both dPul and LIPd on BOLD responses in the activated brain areas was found to be different between the three cognitive tasks the animals were performing. In both animals, effects of electrical microstimulation on BOLD activity were approximately additive and the coefficients resulting from fitting BOLD responses in stimulation and control trials very well resembled the actual pattern of taskdependent stimulation effects on BOLD activation. Although in most cases an additive model fitted BOLD responses as well as or better than a multiplicative model, in some cases the additive model still yielded only poor goodness of fit. This indicates that a simple additive model might not account for more complex microstimulation effects on BOLD signals.

In monkey $\mathrm{C}$, both $\mathrm{dPul}$ and LIPd stimulation led to a consistently stronger enhancement of BOLD responses in both hemispheres when the animal was processing a visual cue and preparing a saccade towards a cued location in the contraversive hemifield, i.e. the hemifield contralateral to the side of stimulation, than when a spatial location in the ipsiversive hemifield was cued. Thus, both dPul and LIPd stimulation may lead to a change in the strength of whole-brain space representations in the activated network with an overrepresentation of the 
contraversive side of visual space. This further suggests that neuronal activity induced by electrical microstimulation is not passively transmitted to brain regions that are mono- or polysynaptically connected to the stimulated structure but stimulation-induced activity may be modulated depending on extent of task responsiveness of the stimulated area. Both dPul and LIP were shown to have stronger representations of visual stimuli and visuomotor actions directed towards visual stimuli in the contralateral hemifield as compared to the ipsilateral side of space (Benevento \& Miller, 1981; Dominguez-Vargas et al., 2017; Patel et al., 2010). Hence, stronger stimulation effects in both hemispheres in the contraversive memory saccade task in our study might result from the stronger functional involvement of the stimulated regions in this specific task.

In monkey B, we also found task-dependent stimulation effects but the pattern of differences in the magnitude of stimulation effects between tasks was less consistent between stimulation in dPul and in LIPd. The magnitude of effects of stimulation in an anterior site in dPul was very similar between tasks while stimulation in a posterior site in dPul showed the weakest effects in the fixation task and led to stronger enhancement of BOLD activity in the ipsiversive memory saccade task than in the contraversive task. Although dPul neurons exhibit stronger firing in visuomotor tasks related to the contralateral side of space at the population level, a substantial portion of cells does not show clear spatial tuning and a smaller subset of neurons even shows ipsilateral tuning (Dominguez-Vargas et al., 2017). Therefore, only small differences between the stimulation effects during different tasks related to different parts of the visual space are not quite surprising. Although dPul cells are known to have a very poor topographic organization (Dominguez-Vargas et al., 2017; Petersen et al., 1985), our data further suggest that the precise location of the stimulation electrode inside $\mathrm{dPul}$ might still determine the strength of dPul stimulation effects on neuronal activity in the activated network in different tasks. Thus, pulvinar cells might show a certain extent of topographic organization but, as previously stated for area LIP, a standard model of topographic organization as used to describe the topography of early sensory and late motor areas might not be suitable to capture the more complex organization of dPul cells (Patel et al., 2014).

Task-dependent effects of LIPd stimulation in monkey B were not congruent with the pattern found in monkey C either. Stimulation in an anterior site in LIPd in monkey B exhibited strongest effects during the fixation task. This finding also supports the hypothesis that the involvement of the stimulated area in a specific task might modulate the strength of stimulation effects in activated brain regions as there is evidence for a foveal representation in anterior LIP (Patel et al., 2014). Hence, the strong effects of stimulation in anterior LIPd found in monkey B may suggest that we stimulated a part of LIP representing the fovea 
which might result in an overrepresentation of the fovea across the whole brain network activated by stimulation. However, strong stimulation effects during fixation were also found for stimulation in posterior LIP so that differences between the effects of LIP stimulation in the two animals might also reflect the functional heterogeneity of area LIP as implicated by electrophysiological recordings from LIP neurons using alternative selection criteria (Premereur et al., 2011). An alternative explanation might be that the control conditions of the contraversive and the ipsiversive memory saccade task already induced very strong neuronal responses in the areas activated by stimulation leaving not much room for further modulation of the response by microstimulation before reaching saturation of the local neuronal activity. Since the ROIs in our study were not defined based on their task responsiveness but based on overall stimulation effects on BOLD responses irrespective of the task, the activated ROIs in our study might not necessarily show similar task responsiveness. This may especially be relevant for areas with topographic organization such as occipitotemporal cortex and FEF (Vanduffel et al., 2014). Future work investigating the relationship between task responsiveness and responsiveness to stimulation could help to further elaborate on this hypothesis and to determine the extent of overlap between task-responsive and stimulationresponsive areas.

\subsubsection{Effects of microstimulation of dPul and LIP on spatial tuning}

To further investigate how dPul and LIP microstimulation affect space representations in the brain, we compared the contraversive selectivity across ROIs in control and stimulation conditions. Consistent with the overrepresentation of the contraversive side of visual space in monkey $\mathrm{C}$, we found a consistent increase in contraversive selectivity, i.e. stronger contraversive tuning during both $\mathrm{dPul}$ and LIPd stimulation in both hemispheres in the same animal. In monkey $\mathrm{B}$, changes in contraversive selectivity were not as consistent across stimulation sites. However, in contrast to changes in contraversive selectivity found in monkey $\mathrm{C}$, stimulation in both posterior $\mathrm{dPul}$ and anterior LIPd led to a significant decrease in contraversive selectivity, i.e. stronger ipsiversive tuning in the right, stimulated hemisphere, the former also including the left hemisphere. Interestingly, stimulation of these two sites also yielded stronger effects on BOLD activity in the ipsiversive memory saccade task than in the contraversive task. Thus, unilateral microstimulation of dPul and LIP does not only differentially enhance neuronal responses during different tasks related to different parts of visual space but, at the same time, it changes the spatial tuning of the activated areas. This may result in a further overrepresentation of one side of the peripheral visual space within the whole activated brain network. 
Finally, we wanted to study the relationship between the spatial tuning of the activated areas and the magnitude of stimulation effects during the different cognitive tasks. In both animals, we found consistent effects of the extent of contraversive selectivity on the strength of the effects of both dPul and LIPd stimulation. In the ipsiversive memory saccade task and in the fixation task greater effects of dPul and LIPd stimulation were found with stronger contraversive tuning of the activated areas. In contrast, in the contraversive task we found stronger effects of dPul stimulation with weaker contraversive tuning of the activated areas in monkey C. Overall, our data may suggest reversed patterns of task-dependent effects of dPul and LIP stimulation for activated areas with contraversive tuning and areas with ipsiversive tuning. More specifically, in areas with contraversive tuning, stimulation of dPul and LIP may strongly enhance neuronal responses to visual cues and motor preparation related to spatial locations in the ipsiversive hemifield. In areas with ipsiversive tuning, by contrast, $\mathrm{dPul}$ and LIP stimulation may lead to strong enhancement of neuronal responses related to visuomotor actions towards the contraversive hemifield. These results speak in favor of the hypothesis that a strong task-related neuronal response in an area of the activated brain network, which may be reflected in a strong spatial tuning, leaves less room for modulation of neuronal activity by stimulation before saturation is approached. As a result, cortical and subcortical microstimulation might lead to the preferential activation of areas that are not strongly involved in the current cognitive task. This hypothesis is supported by another fMRI study finding fMRI activation in visual cortex induced by FEF microstimulation mostly in voxels which were not activated by the presentation of visual stimuli (Premereur et al., 2013). However, additional analysis of the current data is needed to further test this hypothesis.

\subsubsection{Limitations and future directions}

Although BOLD activation patterns resulting from both $\mathrm{dPul}$ and LIP stimulation were consistent between the two animals, further analysis based on BOLD response amplitudes within each ROI should be used to test the robustness of effects revealed by the statistical t maps (Premereur et al., 2015b). Furthermore, our analyses were restricted to positive activation induced by high-frequency electrical microstimulation. However, microstimulation of a brain area can also lead to activity suppression in connected regions, especially when using a lower frequency of current pulses (Logothetis et al., 2010). Thus, the pattern of brain activity, including suppression, resulting from dPul and LIP stimulation at different frequencies could be the objective of future work. Finally, our ROI analyses were based on average BOLD responses within the entire memory period starting with the offset of the visual cue in the memory saccade task or the corresponding time window in the fixation task. Due to the delay of the hemodynamic response, one would expect to find responses related 
to both the presentation of the visual stimulus and working memory and motor preparation processes (Kagan et al., 2010). Hence, further analyses are needed to investigate whether $\mathrm{dPul}$ and LIP stimulation have differential effects on purely visual BOLD responses and BOLD signals related to higher cognitive functions.

\subsubsection{Conclusions}

dPul and LIPd share a functional network comprising dlPFC, the dorsal bank and the fundus of STS, PPC, PCC, somatosensory areas, and primary and extrastriate visual cortex providing evidence that $\mathrm{dPul}$ plays a functional role in the brain network involved in visuospatial attention and visuomotor processing. Importantly, neuronal activity induced by electrical microstimulation was transmitted monosynaptically as well as polysynaptically. Moreover, electrical microstimulation of $\mathrm{dPul}$ and LIPd may change space representations within the activated brain network dependent on the current cognitive task and the spatial tuning of the activated areas. Task-dependent effects of microstimulation may further lead to preferential recruitment of brain regions which are not strongly involved in the current cognitive task.

\section{Acknowledgments}

We thank Elena Spanou for collecting a subset of the MRI data from monkey C, Dr. Sebastian Moeller for sharing the design of the MR-compatible electrode drive, helping with the microstimulation setup, and for valuable suggestions related to fMRI measurements, Jochen Weber for providing support for NeuroElf, and Ira Panolias, Sina Plümer, Leonore Burchardt, Klaus Heisig, and Dirk Prüße for technical support. 



\section{Chapter 4}

\section{General discussion}

In this $\mathrm{PhD}$ thesis I presented data showing that unilateral electrical microstimulation of $\mathrm{dPul}$ biases visuomotor response selection towards responses to contralateral visual stimuli even if the selected response does not yield a reward. This implicates that dPul is involved in the facilitation of visuomotor responses towards contralateral stimuli rather than in filtering contralateral visual distractors. Interestingly, the behavioral outcome of the enhanced contralateral response facilitation due to microstimulation depended on the strength of the signal driven by the contraversive stimulus, i.e. the stimulus contralateral to the side of stimulation. Moreover, using the combination of fMRI and electrical microstimulation in monkeys performing three different cognitive tasks (contraversive memory saccades, ipsiversive memory saccades, or fixation) we were able to identify dPul as a subcortical node of the functional brain network involved in visual attention and visuomotor processing typically described as comprising cortical areas in frontal, parietal, and temporal lobe. More specifically, we found great overlap between the effective connectivity patterns of dPul and LIPd. Importantly, stimulation-induced BOLD activity was not only transmitted to brain regions which are directly connected with pulvinar but also to regions connected through multiple synapses such as areas in the opposite hemisphere. Moreover, the magnitude of BOLD activity enhancement due to stimulation differed between cognitive tasks suggesting that the stimulation-induced activation might be modulated depending on the extent of task responsiveness of the stimulated area. Task-dependent effects of stimulation may also lead to differential changes in space presentations in the whole activated brain network. However, spatial tuning of the activated areas may also determine the strength of stimulation effects on neuronal activity in the different tasks which might result in the preferential recruitment of areas not showing strong involvement in the respective task in the first place.

Electrical microstimulation has often been used in behavioral paradigms to study the causal role of cortical areas such as LIP as well as subcortical regions including dPul and the 
behavioral outcomes of $\mathrm{dPul}$ and LIP stimulation are strikingly similar. LIP microstimulation facilitates saccade responses towards visual stimuli in the receptive field of the stimulated neurons, typically located in the contralateral hemifield, only under conditions of response competition between potential saccade goals presented in opposite hemifields (Dai et al., 2014; Hanks et al., 2006). Previous research (Dominguez-Vargas et al., 2017) and the behavioral study presented in the first chapter of this $\mathrm{PhD}$ thesis yielded very similar findings for dPul stimulation. The great similarity between the causal role of $\mathrm{dPul}$ and LIP is further supported by studies using pharmacological inactivation. Again, inactivation of both LIP (Wardak et al., 2002; Wilke et al., 2012) and dPul (Wilke et al., 2013, 2010) bias saccade responses towards visual stimuli in the ipsilesional hemifield only under conditions of behavioral choice between saccade options presented in opposite hemifields.

However, the combination of microstimulation with neuronal measures to study neuronal correlates of stimulation effects on visuomotor behavior is quite rare (Premereur et al., 2014). The combination of fMRI and electrical microstimulation may help to understand the neuronal mechanisms underlying stimulation-induced changes in behavior. For instance, in monkey $\mathrm{C}$, who served as a subject in both studies presented in this $\mathrm{PhD}$ thesis, unilateral dPul stimulation facilitated saccade responses towards contraversive visual stimuli even when the contraversive stimulus was a visual distractor. In the same subject, dPul stimulation led to a stronger representation of the contraversive side of space in the visuomotor brain network which may be the neuronal mechanism underlying the specific changes in saccade behavior. Similarly, changes in space representations due to LIP microstimulation as indicated in the current fMRI study may be a neuronal correlate of behavioral changes observed in the studies described above. However, our fMRI study did not include experimental conditions resembling competition between multiple response options. Future studies could use the combination of fMRI and electrical microstimulation to study the effects of dPul stimulation on the representation of saccade targets and visual distractors in the visuomotor brain network under conditions of response competition.

It is important to note that it is not possible to infer the directionality of information flow from effective connectivity patterns because electrical stimulation creates both orthodromically and antidromically propagating action potentials (Grill et al., 2008). Moreover, high-frequency stimulation as was used in the two studies presented in this thesis may lead to "neural hijacking", i.e. the stimulation-evoked firing of cortical neurons might block and replace the natural neuronal activity (Griffin et al., 2011). On the other hand, our finding that the strength of stimulation effects in activated areas depended on the current cognitive task supports the hypothesis that the stimulation-induced activity interacts with the existing natural activity. Another limitation that should be taken into account is the observation that 
the typical current strength used in fMRI studies in awake monkeys, ranging from 200 to 300 $\mu \mathrm{A}$ (Moeller et al., 2008; Premereur et al., 2016) is higher than the current intensities used in behavioral studies, which typically do not exceed $75 \mu \mathrm{A}$ (Dai et al., 2014; Fetsch et al., 2014; Pezaris \& Reid, 2007). It is noteworthy, however, that robust effective connectivity patterns were also found using current strengths below $50 \mu \mathrm{A}$ (Ekstrom et al., 2008) and, importantly, in the two studies presented in this $\mathrm{PhD}$ thesis current strengths were matched.

Taken together, our findings suggest that behavioral changes in visuomotor behavior resulting from electrical microstimulation of both $\mathrm{dPul}$ and nodes of the cortical frontotemporoparietal network may result from changes in space representations in the same functional brain network through both corticocortical interactions as well as interactions between $\mathrm{dPul}$ and cortical areas. 



\section{References}

Acuna, C., Cudeiro, J., Gonzalez, F., Alonso, J. M., and Perez, R. (1990). Lateral-posterior and pulvinar reaching cells - comparison with parietal area 5a: a study in behaving macaca nemestrina monkeys. Exp Brain Res, 82:158-166.

Acuna, C., Gonzalez, F., and Dominguez, R. (1983). Sensorimotor unit activity related to intention in the pulvinar of behaving cebus apella monkeys. Exp Brain Res, 52:411-422.

Adams, M. M., Hof, P. R., Gattass, R., Webster, M. J., and Ungerleider, L. G. (2000). Visual cortical projections and chemoarchitecture of macaque monkey pulvinar. J Comp Neurol, 419(3):377-393.

Andersen, R. A., Asanuma, C., Essick, G., and Siegel, R. M. (1990a). Corticocortical connections of anatomically and physiologically defined subdivisions within the inferior parietal lobule. J Comp Neurol, 296(1):65-113.

Andersen, R. A., Bracewell, R. M., Barash, S., Gnadt, J. W., and Fogassi, L. (1990b). Eye position effects on visual, memory, and saccade-related activity in areas lip and 7a of macaque. J Neurosci, 10(4):1176-1196.

Arend, I., Machado, L., Ward, R., McGrath, M., Ro, T., and Rafal, R. D. (2008). The role of the human pulvinar in visual attention and action: evidence from temporal-order judgment, saccade decision, and antisaccade tasks. Prog Brain Res, 171:475-83.

Asanuma, C., Andersen, R. A., and Cowan, W. M. (1985). The thalamic relations of the caudal inferior parietal lobule and the lateral prefrontal cortex in monkeys: divergent cortical projections from cell clusters in the medial pulvinar nucleus. J Comp Neurol, 241(3):357-81.

Baleydier, C. and Mauguiere, F. (1987). Network organization of the connectivity between parietal area 7, posterior cingulate cortex and medial pulvinar nucleus: a double fluorescent tracer study in monkey. Exp Brain Res, 66(2).

Barbas, H., Henion, T. H., and Dermon, C. R. (1991). Diverse thalamic projections to the prefrontal cortex in the rhesus monkey. J Comp Neurol, 313(1):65-94.

Ben Hamed, S., Duhamel, J. R., Bremmer, F., and Graf, W. (2001). Representation of the visual field in the lateral intraparietal area of macaque monkeys: a quantitative receptive field analysis. Exp Brain Res, 140(2):127-44.

Bender, D. B. (1981). Retinotopic organization of macaque pulvinar. J Neurophysiol, 46(3):672-693. 
Bender, D. B. (1982). Receptive-field properties of neurons in the macaque inferior pulvinar. J Neurophysiol, 48(1):1-17.

Bender, D. B. and Baizer, J. S. (1990). Saccadic eye movements following kainic acid lesions of the pulvinar in monkeys. Exp Brain Res, 79:467-478.

Bender, D. B. and Butter, C. M. (1987). Comparison of the effects of superior colliculus and pulvinar lesions on visual search and tachistoscopic pattern discrimination in monkeys. Exp Brain Res, 69:140-154.

Bender, D. B. and Youakim, M. (2001). Effect of attentive fixation in macaque thalamus and cortex. J Neurophysiol, 85:219-234.

Benevento, L. A. and Fallon, J. H. (1975). The ascending projections of the superior colliculus in the rhesus monkey (macaca mulatta). J Comp Neurol, 160(3):339-61.

Benevento, L. A. and Miller, J. (1981). Visual responses of single neurons in the caudal lateral pulvinar of the macaque monkey. J Neurosci, 1(11):1268-1278.

Benevento, L. A. and Port, J. D. (1995). Single neurons with both form/color differential responses and saccade-related responses in the nonretinotopic pulvinar of the behaving macaque monkey. Visual Neuroscience, 12:523-544.

Benevento, L. A. and Rezak, M. (1976). The cortical projections of the inferior pulvinar and adjacent lateral pulvinar in the rhesus monkey (macaca mulatta): An autoradiographic study. Brain Res, 108(1):1-24.

Benjamini, Y. and Hochberg, Y. (1995). Controlling the false discovery rate: A practical and powerful approach to multiple testing. Journal of the Royal Statistical Society. Series B (Methodological), 57(1):289-300.

Berman, R. A., Cavanaugh, J., McAlonan, K., and Wurtz, R. H. (2016). A circuit for saccadic suppression in the primate brain. J Neurophysiol, page jn 006792016.

Berman, R. A. and Wurtz, R. H. (2010). Functional identification of a pulvinar path from superior colliculus to cortical area mt. J Neurosci, 30(18):6342-54.

Bisley, J. W. and Goldberg, M. E. (2003). Neuronal activity in the lateral intraparietal area and spatial attention. Science, 299(5603):81-6.

Blatt, G. J., Andersen, R. A., and Stoner, G. R. (1990). Visual receptive field organization and cortico-cortical connections of the lateral intraparietal area (area lip) in the macaque. J Comp Neurol, 299(4):421-45.

Blum, B. (1984). Specific and non-specific inhibition induced by lp-pulvinar inputs to area 7 of the rhesus monkey. Archives Italiennes de Biologie, 122:237-248.

Blum, B. (1985). Enhancement of visual responses of area 7 neurons by electrical preconditioning stimulation of lp-pulvinar nuclei of the monkey. Exp Brain Res, 59:434-440.

Bos, J. and Benevento, L. A. (1975). Projections of the medial pulvinar to orbital cortex and frontal eye fields in the rhesus monkey (macaca mulatta). Experimental Neurology, 49(2):487-496. 
Bourne, J. A. and Morrone, M. C. (2017). Plasticity of visual pathways and function in the developing brain: Is the pulvinar a crucial player? Front Syst Neurosci, 11:3.

Brainard, D. H. (1997). The psychophysics toolbox. Spatial Vision, 10(4):433-436.

Buckwalter, J. A., Parvizi, J., Morecraft, R. J., and van Hoesen, G. W. (2008). Thalamic projections to the posteromedial cortex in the macaque. J Comp Neurol, 507(5):1709-33.

Burton, H. and Jones, E. G. (1976). The posterior thalamic region and its cortical projection in new world and old world monkeys. J Comp Neurol, 168(2):249-301.

Calabrese, E., Badea, A., Coe, C. L., Lubach, G. R., Shi, Y., Styner, M. A., and Johnson, G. A. (2015). A diffusion tensor mri atlas of the postmortem rhesus macaque brain. Neuroimage, 117:408-16.

Cappe, C., Morel, A., Barone, P., and Rouiller, E. M. (2009). The thalamocortical projection systems in primate: an anatomical support for multisensory and sensorimotor interplay. Cereb Cortex, 19(9):2025-37.

Cappe, C., Morel, A., and Rouiller, E. M. (2007). Thalamocortical and the dual pattern of corticothalamic projections of the posterior parietal cortex in macaque monkeys. Neuroscience, $146(3): 1371-87$.

Caspari, N., Janssens, T., Mantini, D., Vandenberghe, R., and Vanduffel, W. (2015). Covert shifts of spatial attention in the macaque monkey. J Neurosci, 35(20):7695-714.

Chalupa, L. M., Coyle, R. S., and Lindsley, D. B. (1976). Effect of pulvinar lesions on visual pattern discrimination in monkeys. J Neurophysiol, 39(2):354-369.

Cheney, P. D., Griffin, D. M., and Van Acker III, G. M. (2013). Neural hijacking: action of high-frequency electrical stimulation on cortical circuits. Neuroscientist, 19(5):434-41.

Christopoulos, V. N., Bonaiuto, J., Kagan, I., and Andersen, R. A. (2015). Inactivation of parietal reach region affects reaching but not saccade choices in internally guided decisions. J Neurosci, 35(33):11719-28.

Cicmil, N., Cumming, B. G., Parker, A. J., and Krug, K. (2015). Reward modulates the effect of visual cortical microstimulation on perceptual decisions. Elife, 4:e07832.

Cohen, A., Ivry, R. B., Rafal, R. D., and Kohn, C. (1995). Activating response codes by stimuli in the neglected visual field. Neuropsychology, 9(2):165-173.

Colby, C. L., Duhamel, J. R., and Goldberg, M. E. (1996). Visual, presaccadic, and cognitive activation of single neurons in monkey lateral intraparietal area. $J$ Neurophysiol, 76(5):2841.

Contini, M., Baccarini, M., Borra, E., Gerbella, M., Rozzi, S., and Luppino, G. (2010). Thalamic projections to the macaque caudal ventrolateral prefrontal areas $45 \mathrm{a}$ and $45 \mathrm{~b}$. Eur J Neurosci, 32(8):1337-53.

Corbetta, M. (1998). Frontoparietal cortical networks for directing attention and the eye to visual locations: Identical, independent, or overlapping neural systems? Proc Natl Acad Sci U S A, 95:831-838. 
Dai, J., Brooks, D. I., and Sheinberg, D. L. (2014). Optogenetic and electrical microstimulation systematically bias visuospatial choice in primates. Curr Biol, 24(1):63-9.

Danziger, S., Ward, R., Owen, V., and Rafal, R. (2002). The effects of unilateral pulvinar damage in humans on reflexive orienting and filtering of irrelevant information. Behavioural Neurology, 13(3-4):95-104.

Danziger, S., Ward, R., Owen, V., and Rafal, R. (2004). Contributions of the human pulvinar to linking vision and action. Cognitive, Affective, and Behavioral Neuroscience, 4(1):89-99.

Darian-Smith, C. and Darian-Smith, I. (1993). Thalamic projections to areas 3a, 3b, and 4 in the sensorimotor cortex of the mature and infant macaque monkey. J Comp Neurol, 335(2):173-99.

Desimone, R., Wessinger, M., Thomas, L., and Schneider, W. (1990). Attentional control of visual perception: Cortical and subcortical mechanisms. Cold Spring Harbor Symposia on Quantitative Biology, 55(0):963-971.

Dominguez-Vargas, A. U., Schneider, L., Wilke, M., and Kagan, I. (2017). Electrical microstimulation of the pulvinar biases saccade choices and reaction times in a timedependent manner. J Neurosci, 37(8):2234-2257.

Duncan, J., Humphreys, G., and Ward, R. (1997). Competitive brain activity in visual attention. Current Opinion in Neurobiology, 7:255-261.

Ekstrom, L. B., Roelfsema, P. R., Arsenault, J. T., Bonmassar, G., and Vanduffel, W. (2008). Bottom-up dependent gating of frontal signals in early visual cortex. Science, 321(5887):414-7.

Eriksen, B. A. and Eriksen, C. W. (1974). Effects of noise letters upon the identification of a target letter in a nonsearch task. Perception and Psychophysics, 16(1):143-149.

Felleman, D. J. and Van Essen, D. C. (1991). Distributed hierarchical processing in the primate cerebral cortex. Cereb Cortex, 1:1-47.

Fetsch, C. R., Kiani, R., Newsome, W. T., and Shadlen, M. N. (2014). Effects of cortical microstimulation on confidence in a perceptual decision. Neuron, 83(4):797-804.

Field, C. B., Johnston, K., Gati, J. S., Menon, R. S., and Everling, S. (2008). Connectivity of the primate superior colliculus mapped by concurrent microstimulation and event-related fmri. PLoS One, 3(12):e3928.

Findlay, J. M. and Walker, R. (1999). A model of saccade generation based on parallel processing and competitive inhibition. Behavioral and Brain Sciences, 22:661-721.

Fischer, J. and Whitney, D. (2012). Attention gates visual coding in the human pulvinar. Nat Commun, 3:1051.

Friedman, D. P. and Murray, E. A. (1986). Thalamic connectivity of the second somatosensory area and neighboring somatosensory fields of the lateral sulcus of the macaque. J Comp Neurol, 252(3):348-73. 
Fries, W. (1984). Cortical projections to the superior colliculus in the macaque monkey: a retrograde study using horseradish peroxidase. J Comp Neurol, 230(1):55-76.

Grieve, K. L., Acuna, C., and Cudeiro, J. (2000). The primate pulvinar nuclei:vision and action. Trends Neurosci, 23:35-39.

Griffin, D. M., Hudson, H. M., Belhaj-Saif, A., and Cheney, P. D. (2011). Hijacking cortical motor output with repetitive microstimulation. J Neurosci, 31(37):13088-96.

Grill, W. M., Cantrell, M. B., and Robertson, M. S. (2008). Antidromic propagation of action potentials in branched axons: implications for the mechanisms of action of deep brain stimulation. J Comput Neurosci, 24(1):81-93.

Hakamata, Y., Sato, E., Komi, S., Moriguchi, Y., Izawa, S., Murayama, N., Hanakawa, T., Inoue, Y., and Tagaya, H. (2016). The functional activity and effective connectivity of pulvinar are modulated by individual differences in threat-related attentional bias. Sci Rep, 6:34777.

Hanks, T. D., Ditterich, J., and Shadlen, M. N. (2006). Microstimulation of macaque area lip affects decision-making in a motion discrimination task. Nat Neurosci, 9(5):682-9.

Hardy, S. G. P. and Lynch, J. C. (1992). The spatial distribution of pulvinar neurons that project to two subregions of the inferior parietal lobule in the macaque. Cereb Cortex, $2(3): 217-230$.

Harting, J. K., Hall, W. C., and Diamond, I. T. (1972). Evolution of the pulvinar (part 1 of 2). Brain, Behavior and Evolution, 6(1-6):424-438.

Hwang, E. J., Hauschild, M., Wilke, M., and Andersen, R. A. (2014). Spatial and temporal eye-hand coordination relies on the parietal reach region. J Neurosci, 34(38):12884-92.

Jones, E. G. and Burton, H. (1976). A projection from the medial pulvinar to the amygdala in primates. Brain Res, 104:142-147.

Jones, E. G., Wise, S. P., and Coulter, J. D. (1979). Differential thalamic relationships of sensory-motor and parietal cortical fields in monkeys. J Comp Neurol, 183(4):833-81.

Kaas, J. H. and Lyon, D. C. (2007). Pulvinar contributions to the dorsal and ventral streams of visual processing in primates. Brain Res Rev, 55(2):285-96.

Kagan, I., Iyer, A., Lindner, A., and Andersen, R. A. (2010). Space representation for eye movements is more contralateral in monkeys than in humans. Proc Natl Acad Sci U S A, 107(17):7933-8.

Katz, L. N., Yates, J. L., Pillow, J. W., and Huk, A. C. (2016). Dissociated functional significance of decision-related activity in the primate dorsal stream. Nature, 535(7611):285-8.

Kievit, J. and Kuypers, H. G. J. M. (1977). Organization of the thalamo-cortical connexions to the frontal lobe in the rhesus monkey. Exp Brain Res, 29-29(3-4).

Komura, Y., Nikkuni, A., Hirashima, N., Uetake, T., and Miyamoto, A. (2013). Responses of pulvinar neurons reflect a subject's confidence in visual categorization. Nat Neurosci, 16(6):749-55. 
Koyama, M., Hasegawa, I., Osada, T., Adachi, Y., Nakahara, K., and Miyashita, Y. (2004). Functional magnetic resonance imaging of macaque monkeys performing visually guided saccade tasks: Comparison of cortical eye fields with humans. Neuron, 41:795-807.

Kubanek, J., Li, J. M., and Snyder, L. H. (2015). Motor role of parietal cortex in a monkey model of hemispatial neglect. Proc Natl Acad Sci U S A, 112(16):E2067-72.

Kuenzle, H. and Akert, K. (1977). Efferent connections of cortical, area 8 (frontal eye field) in macaca fascicularis. a reinvestigation using the autoradiographic technique. J Comp Neurol, 173(1):147-64.

LaBerge, D. and Buchsbaum, M. S. (1990). Positron emission tomographic measurements of pulvinar activity during an attention task. J Neurosci, 10(2):613-619.

Le, Q. V., Isbell, L. A., Matsumoto, J., Le, V. Q., Nishimaru, H., Hori, E., Maior, R. S., Tomaz, C., Ono, T., and Nishijo, H. (2016). Snakes elicit earlier, and monkey faces, later, gamma oscillations in macaque pulvinar neurons. Sci Rep, 6:20595.

Leiby III, C. C., Bender, D. B., and Butter, C. M. (1982). Localization and detection of visual stimuli in monkeys with pulvinar lesions. Exp Brain Res, 48:449-454.

Lewis, J. W. and Van Essen, D. C. (2000). Corticocortical connections of visual, sensorimotor, and multimodal processing areas in the parietal lobe of the macaque monkey. J Comp Neurol, 428(1):112-137.

Logothetis, N. K., Augath, M., Murayama, Y., Rauch, A., Sultan, F., Goense, J., Oeltermann, A., and Merkle, H. (2010). The effects of electrical microstimulation on cortical signal propagation. Nat Neurosci, 13(10):1283-91.

Luppino, G., Calzavara, R., Rozzi, S., and Matelli, M. (2001). Projections from the superior temporal sulcus to the agranular frontal cortex in the macaque. Eur J Neurosci, 14(6):10351040 .

Luppino, G., Rozzi, S., Calzavara, R., and Matelli, M. (2003). Prefrontal and agranular cingulate projections to the dorsal premotor areas $\mathrm{f} 2$ and $\mathrm{f} 7$ in the macaque monkey. Eur $J$ Neurosci, 17(3):559-578.

Matsui, T., Koyano, K. W., Tamura, K., Osada, T., Adachi, Y., Miyamoto, K., Chikazoe, J., Kamigaki, T., and Miyashita, Y. (2012). Fmri activity in the macaque cerebellum evoked by intracortical microstimulation of the primary somatosensory cortex: evidence for polysynaptic propagation. PLoS One, 7(10):e47515.

Matsui, T., Tamura, K., Koyano, K. W., Takeuchi, D., Adachi, Y., Osada, T., and Miyashita, Y. (2011). Direct comparison of spontaneous functional connectivity and effective connectivity measured by intracortical microstimulation: an fmri study in macaque monkeys. Cereb Cortex, 21(10):2348-56.

Michael, G. A. and Buron, V. (2005). The human pulvinar and stimulus-driven attentional control. Behav Neurosci, 119(5):1353-67.

Moeller, S., Freiwald, W. A., and Tsao, D. Y. (2008). Patches with links: a unified system for processing faces in the macaque temporal lobe. Science, 320(5881):1355-9. 
Morecraft, R. J., Geula, C., and Mesulam, M. M. (1992). Cytoarchitecture and neural afferents of orbitofrontal cortex in the brain of the monkey. J Comp Neurol, 323(3):34158.

Morel, A., Liu, J., Wannier, T., Jeanmonod, D., and Rouiller, E. M. (2005). Divergence and convergence of thalamocortical projections to premotor and supplementary motor cortex: a multiple tracing study in the macaque monkey. Eur J Neurosci, 21(4):1007-29.

Mufson, E. J. and Mesulam, M. M. (1984). Thalamic connections of the insula in the rhesus monkey and comments on the paralimbic connectivity of the medial pulvinar nucleus. $J$ Comp Neurol, 227(1):109-20.

O'Brien, B. J., Abel, P. L., and Olavarria, J. F. (2001). The retinal input to calbindin-d28kdefined subdivisions in macaque inferior pulvinar. Neuroscience Letters, 312(3):145-148.

Ohayon, S. and Tsao, D. Y. (2012). Mr-guided stereotactic navigation. J Neurosci Methods, 204(2):389-97.

Patel, G. H., Kaplan, D. M., and Snyder, L. H. (2014). Topographic organization in the brain: searching for general principles. Trends Cogn Sci, 18(7):351-63.

Patel, G. H., Shulman, G. L., Baker, J. T., Akbudak, E., Snyder, A. Z., Snyder, L. H., and Corbetta, M. (2010). Topographic organization of macaque area lip. Proc Natl Acad Sci U $S$ A, 107(10):4728-33.

Petersen, S. E., Robinson, D. L., and Keys, W. (1985). Pulvinar nuclei of the behaving rhesus monkey: Visual responses and their modulation. J Neurophysiol, 54(4):867-886.

Petersen, S. E., Robinson, D. L., and Morris, J. D. (1987). Contributions of the pulvinar to visual spatial attention. Neuropsychologia, 25(1A):97-105.

Petkov, C. I., Kikuchi, Y., Milne, A. E., Mishkin, M., Rauschecker, J. P., and Logothetis, N. K. (2015). Different forms of effective connectivity in primate frontotemporal pathways. Nat Commun, 6:6000.

Pezaris, J. S. and Reid, R. C. (2007). Demonstration of artificial visual percepts generated through thalamic microstimulation. Proc Natl Acad Sci U S A, 104(18):7670-5.

Platt, M. L. and Glimcher, P. W. (1997). Responses of intraparietal neurons to saccadic targets and visual distractors. J Neurophysiol, 78:1574-1589.

Platt, M. L. and Glimcher, P. W. (1998). Response fields of intraparietal neurons quantified with multiple saccadic targets. Exp Brain Res, 121(1):65-75.

Pons, T. P. and Kaas, J. H. (1985). Connections of area 2 of somatosensory cortex with the anterior pulvinar and subdivisions of the ventroposterior complex in macaque monkeys. $J$ Comp Neurol, 240(1):16-36.

Premereur, E., Janssen, P., and Vanduffel, W. (2013). Fef-microstimulation causes taskdependent modulation of occipital fmri activity. Neuroimage, 67:42-50.

Premereur, E., Janssen, P., and Vanduffel, W. (2015a). Effector specificity in macaque frontal and parietal cortex. $J$ Neurosci, 35(8):3446-59. 
Premereur, E., Taubert, J., Janssen, P., Vogels, R., and Vanduffel, W. (2016). Effective connectivity reveals largely independent parallel networks of face and body patches. Curr Biol.

Premereur, E., Van Dromme, I. C., Romero, M. C., Vanduffel, W., and Janssen, P. (2015b). Effective connectivity of depth-structure-selective patches in the lateral bank of the macaque intraparietal sulcus. PLoS Biol, 13(2):e1002072.

Premereur, E., Vanduffel, W., and Janssen, P. (2011). Functional heterogeneity of macaque lateral intraparietal neurons. J Neurosci, 31(34):12307-17.

Premereur, E., Vanduffel, W., and Janssen, P. (2014). The effect of fef microstimulation on the responses of neurons in the lateral intraparietal area. J Cogn Neurosci, 26(8):1672-84.

Prevosto, V., Graf, W., and Ugolini, G. (2009). Posterior parietal cortex areas mip and lipv receive eye position and velocity inputs via ascending preposito-thalamo-cortical pathways. Eur J Neurosci, 30(6):1151-61.

Price, J. L. and Amaral, D. G. (1981). An autoradiographic study of the projections of the central nucleus of the monkey amygdala. J Neurosci, 1(11):1242-1259.

Prins, N. and Kingdom, F. A. A. (2009). Palamedes: Matlab routines for analyzing psychophysical data.

Purushothaman, G., Marion, R., Li, K., and Casagrande, V. A. (2012). Gating and control of primary visual cortex by pulvinar. Nat Neurosci, 15(6):905-12.

Rafal, R., Gershberg, F., Egly, R., Ivry, R., Kingstone, A., and Ro, T. (1996). Response channel activation and the lateral prefrontal cortex. Neuropsychologia, 34(12):1197-1202.

Rafal, R. D. and Posner, M. I. (1987). Deficits in human visual spatial attention following thalamic lesions. Proc Natl Acad Sci U S A, 84:7349-7353.

Ro, T., Cohen, A., Ivry, R. B., and Rafal, R. D. (1998). Response channel activation and the temporoparietal junction. Brain Cogn, 37(3):461-76.

Robinson, D. L., Petersen, S. E., and Keys, W. (1986). Saccade-related and visual activities in the pulvinar nuclei of the behaving rhesus monkey. Exp Brain Res, 62:625-634.

Rockland, K. S., Andresen, J., Cowie, R. J., and Robinson, D. L. (1999). Single axon analysis of pulvinocortical connections to several visual areas in the macaque. J Comp Neurol, 406(2):221-250.

Rohlfing, T., Kroenke, C. D., Sullivan, E. V., Dubach, M. F., Bowden, D. M., Grant, K. A., and Pfefferbaum, A. (2012). The inia19 template and neuromaps atlas for primate brain image parcellation and spatial normalization. Front Neuroinform, 6:27.

Romanski, L. M., Giguere, M., Bates, J. F., and Goldman-Rakic, P. S. (1997). Topographic organization of medial pulvinar connections with the prefrontal cortex in the rhesus monkey. J Comp Neurol, 379(3):313-332. 
Saalmann, Y. B., Pinsk, M. A., Wang, L., Li, X., and Kastner, S. (2012). The pulvinar regulates information transmission between cortical areas based on attention demands. Science, 337(6095):753-6.

Saleem, K. S. and Logothetis, N. (2007). A combined MRI and histology atlas of the rhesus monkey brain in stereotaxic coordinates. Elsevier/Academic Press, UK, 1st edition.

Schmahmann, J. D. and Pandya, D. N. (1990). Anatomical investigation of projections from thalamus to posterior parietal cortex in the rhesus monkey: a wga-hrp and fluorescent tracer study. J Comp Neurol, 295(2):299-326.

Shibata, H. and Yukie, M. (2003). Differential thalamic connections of the posteroventral and dorsal posterior cingulate gyrus in the monkey. Eur J Neurosci, 18(6):1615-1626.

Shipp, S. (2001). Corticopulvinar connections of areas v5, v4, and v3 in the macaque monkey: a dual model of retinal and cortical topographies. J Comp Neurol, 439(4):469-90.

Shipp, S. (2003). The functional logic of cortico-pulvinar connections. Philos Trans R Soc Lond B Biol Sci, 358(1438):1605-24.

Shipp, S. and Zeki, S. (1995). Segregation and convergence of specialised pathways in macaque monkey visual cortex. J Anat, 187:547-562.

Snow, J. C., Allen, H. A., Rafal, R. D., and Humphreys, G. W. (2009). Impaired attentional selection following lesions to human pulvinar: evidence for homology between human and monkey. Proc Natl Acad Sci U S A, 106(10):4054-9.

Soares, J. G., Diogo, A. C., Fiorani, M., Souza, A. P., and Gattass, R. (2004). Effects of inactivation of the lateral pulvinar on response properties of second visual area cells in cebus monkeys. Clin Exp Pharmacol Physiol, 31(9):580-90.

Sommer, M. A. and Wurtz, R. H. (1998). Frontal eye field neurons orthodromically activated from the superior colliculus. J Neurophysiol, 80:3331-3333.

Stepniewska, I., Qi, H.-X., and Kaas, J. H. (2000). Projections of the superior colliculus to subdivisions of the inferior pulvinar in new world and old world monkeys. Visual Neuroscience, 17:529-549.

Strumpf, H., Mangun, G. R., Boehler, C. N., Stoppel, C., Schoenfeld, M. A., Heinze, H. J., and Hopf, J. M. (2013). The role of the pulvinar in distractor processing and visual search. Hum Brain Mapp, 34(5):1115-32.

Sultan, F., Augath, M., Murayama, Y., Tolias, A. S., and Logothetis, N. (2011). esfmri of the upper sts: further evidence for the lack of electrically induced polysynaptic propagation of activity in the neocortex. Magn Reson Imaging, 29(10):1374-81.

Suzuki, M. and Gottlieb, J. (2013). Distinct neural mechanisms of distractor suppression in the frontal and parietal lobe. Nat Neurosci, 16(1):98-104.

Takahara, D., Inoue, K., Hirata, Y., Miyachi, S., Nambu, A., Takada, M., and Hoshi, E. (2012). Multisynaptic projections from the ventrolateral prefrontal cortex to the dorsal premotor cortex in macaques - anatomical substrate for conditional visuomotor behavior. Eur J Neurosci, 36(10):3365-75. 
Takayama, Y., Sugishita, M., Kido, T., Ogawa, M., Fukuyama, H., and Akiguchi, I. (1994). Impaired stereoacuity due to a lesion in the left pulvinar. Journal of Neurology, Neurosurgery and Psychiatry, 57(5):652-654.

Tanne-Gariepy, J., Rouiller, E. M., and Boussaoud, D. (2002). Parietal inputs to dorsal versus ventral premotor areas in the macaque monkey: evidence for largely segregated visuomotor pathways. Exp Brain Res, 145(1):91-103.

Thier, P. and Andersen, R. A. (1998). Electrical microstimulation distinguishes distinct saccade-related areas in the posterior parietal cortex. J Neurophysiol, 80:1713-1735.

Tolias, A. S., Sultan, F., Augath, M., Oeltermann, A., Tehovnik, E. J., Schiller, P. H., and Logothetis, N. K. (2005). Mapping cortical activity elicited with electrical microstimulation using fmri in the macaque. Neuron, 48(6):901-11.

Trojanowski, J. Q. and Jacobson, S. (1976). Areal and laminar distribution of some pulvinar cortical efferents in rhesus monkey. J Comp Neurol, 169(3):371-92.

Ungerleider, L. G., Desimone, R., Galkin, T. W., and Mishkin, M. (1984). Subcortical projections of area mt in the macaque. J Comp Neurol, 223(3):368-86.

Ungerleider, L. G., Galkin, T. W., Desimone, R., and Gattass, R. (2008). Cortical connections of area v4 in the macaque. Cereb Cortex, 18(3):477-99.

Ungerleider, L. G., Galkin, T. W., Desimone, R., and Gattass, R. (2014). Subcortical projections of area v2 in the macaque. J Cogn Neurosci, 26(6):1220-33.

Ungerleider, L. G., Ganz, L., and Pribram, K. H. (1977). Size constancy in rhesus monkeys: Effects of pulvinar, prestriate, and inferotemporal lesions. Exp Brain Res, 27-27(3-4).

Van der Stigchel, S., Arend, I., van Koningsbruggen, M. G., and Rafal, R. D. (2010). Oculomotor integration in patients with a pulvinar lesion. Neuropsychologia, 48(12):3497504.

Van Le, Q., Isbell, L. A., Matsumoto, J., Nguyen, M., Hori, E., Maior, R. S., Tomaz, C., Tran, A. H., Ono, T., and Nishijo, H. (2013). Pulvinar neurons reveal neurobiological evidence of past selection for rapid detection of snakes. Proc Natl Acad Sci U S A, 110(47):19000-5.

Vanduffel, W., Zhu, Q., and Orban, G. A. (2014). Monkey cortex through fmri glasses. Neuron, 83(3):533-50.

Vanni, M. P., Thomas, S., Petry, H. M., Bickford, M. E., and Casanova, C. (2015). Spatiotemporal profile of voltage-sensitive dye responses in the visual cortex of tree shrews evoked by electric microstimulation of the dorsal lateral geniculate and pulvinar nuclei. $J$ Neurosci, 35(34):11891-6.

Villeneuve, M. Y., Kupers, R., Gjedde, A., Ptito, M., and Casanova, C. (2005). Pattern-motion selectivity in the human pulvinar. Neuroimage, 28(2):474-80.

Ward, R. and Danziger, S. (2005). Selective attention and response control following damage to the human pulvinar, book section 14, pages 325-350. Psychology Press, New York. 
Ward, R., Danziger, S., and Bamford, S. (2005). Response to visual threat following damage to the pulvinar. Curr Biol, 15(6):571-3.

Ward, R., Danziger, S., Owen, V., and Rafal, R. (2002). Deficits in spatial coding and feature binding following damage to spatiotopic maps in the human pulvinar. Nat Neurosci, 5(2):99-100.

Wardak, C., Olivier, E., and Duhamel, J.-R. (2002). Saccadic target selection deficits after lateral intraparietal area inactivation in monkeys. J Neurosci, 22(22):9877-9884.

Webster, M. J., Bachevalier, J., and Ungerleider, L. G. (1993). Subcortical connections of inferior temporal areas te and teo in macaque monkeys. J Comp Neurol, 335(1):73-91.

Webster, M. J., Bachevalier, J., and Ungerleider, L. G. (1995). Transient subcortical connections of inferior temporal areas te and teo in infant macaque monkeys. J Comp Neurol, 352(2):213-26.

Wilke, M., Kagan, I., and Andersen, R. A. (2012). Functional imaging reveals rapid reorganization of cortical activity after parietal inactivation in monkeys. Proc Natl Acad Sci U S A, 109(21):8274-9.

Wilke, M., Kagan, I., and Andersen, R. A. (2013). Effects of pulvinar inactivation on spatial decision-making between equal and asymmetric reward options. J Cogn Neurosci, 25(8):1270-83.

Wilke, M., Kagan, I., and Andersen, R. A. (in revision). Context-specific effects of pulvinar inactivation on fmri signals during spatial decision making.

Wilke, M., Turchi, J., Smith, K., Mishkin, M., and Leopold, D. A. (2010). Pulvinar inactivation disrupts selection of movement plans. J Neurosci, 30(25):8650-9.

Yeterian, E. H. and Pandya, D. N. (1985). Corticothalamic connections of the posterior parietal cortex in the rhesus monkey. J Comp Neurol, 237(3):408-26.

Yeterian, E. H. and Pandya, D. N. (1989). Thalamic connections of the cortex of the superior temporal sulcus in the rhesus monkey. J Comp Neurol, 282(1):80-97.

Yeterian, E. H. and Pandya, D. N. (1991). Corticothalamic connections of the superior temporal sulcus in rhesus monkeys. Exp Brain Res, 83(2).

Yeterian, E. H. and Pandya, D. N. (1997). Corticothalamic connections of extrastriate visual areas in rhesus monkeys. J Comp Neurol, 378(4):562-585.

Yeterian, E. H., Pandya, D. N., Tomaiuolo, F., and Petrides, M. (2012). The cortical connectivity of the prefrontal cortex in the monkey brain. Cortex, 48(1):58-81.

Zhong, Y. M. and Rockland, K. S. (2003). Inferior parietal lobule projections to anterior inferotemporal cortex (area te) in macaque monkey. Cereb Cortex, 13(5):527-40.

Zhou, H., Schafer, R. J., and Desimone, R. (2016). Pulvinar-cortex interactions in vision and attention. Neuron, 89(1):209-20. 

Appendix

Abbreviations 


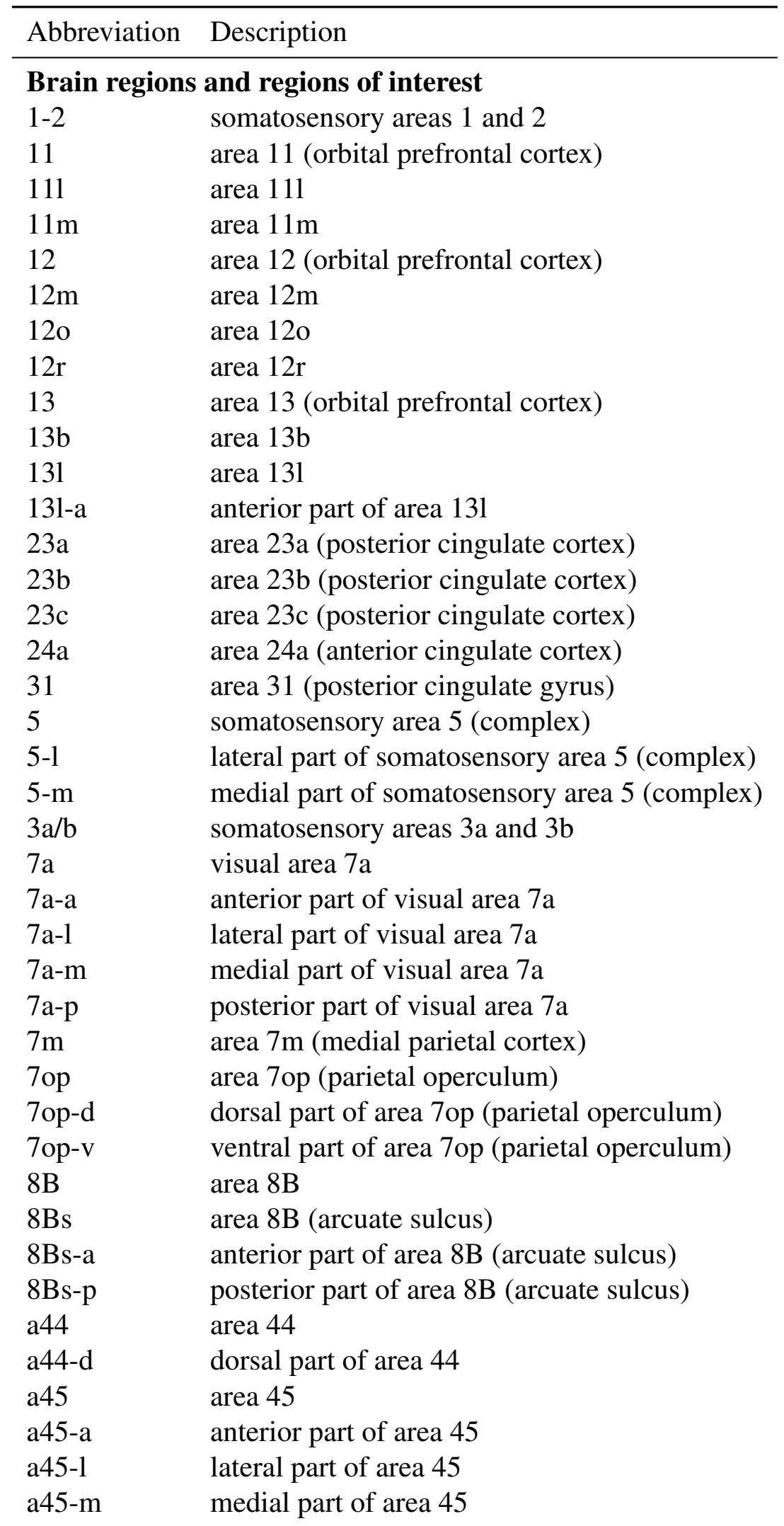




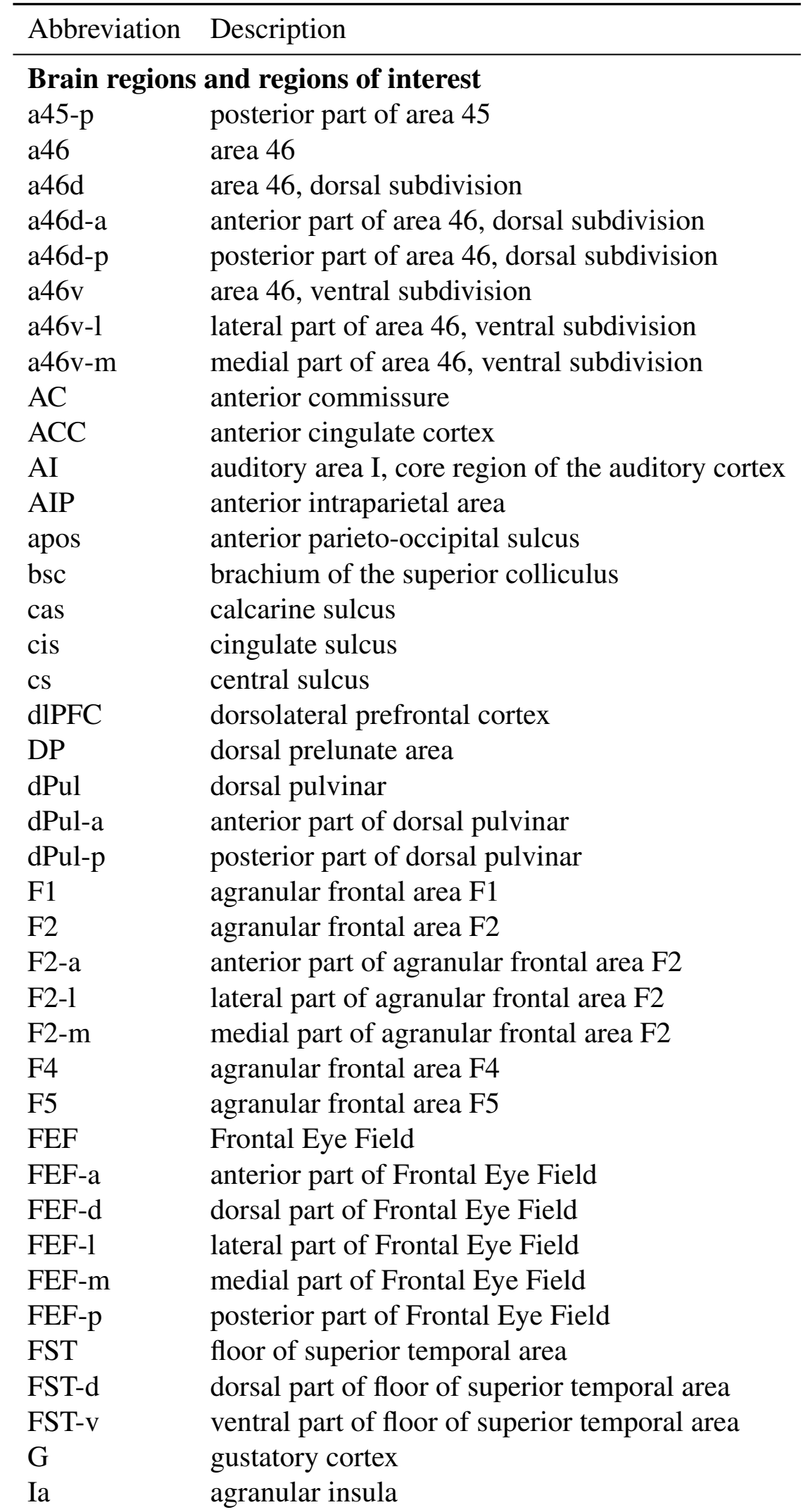




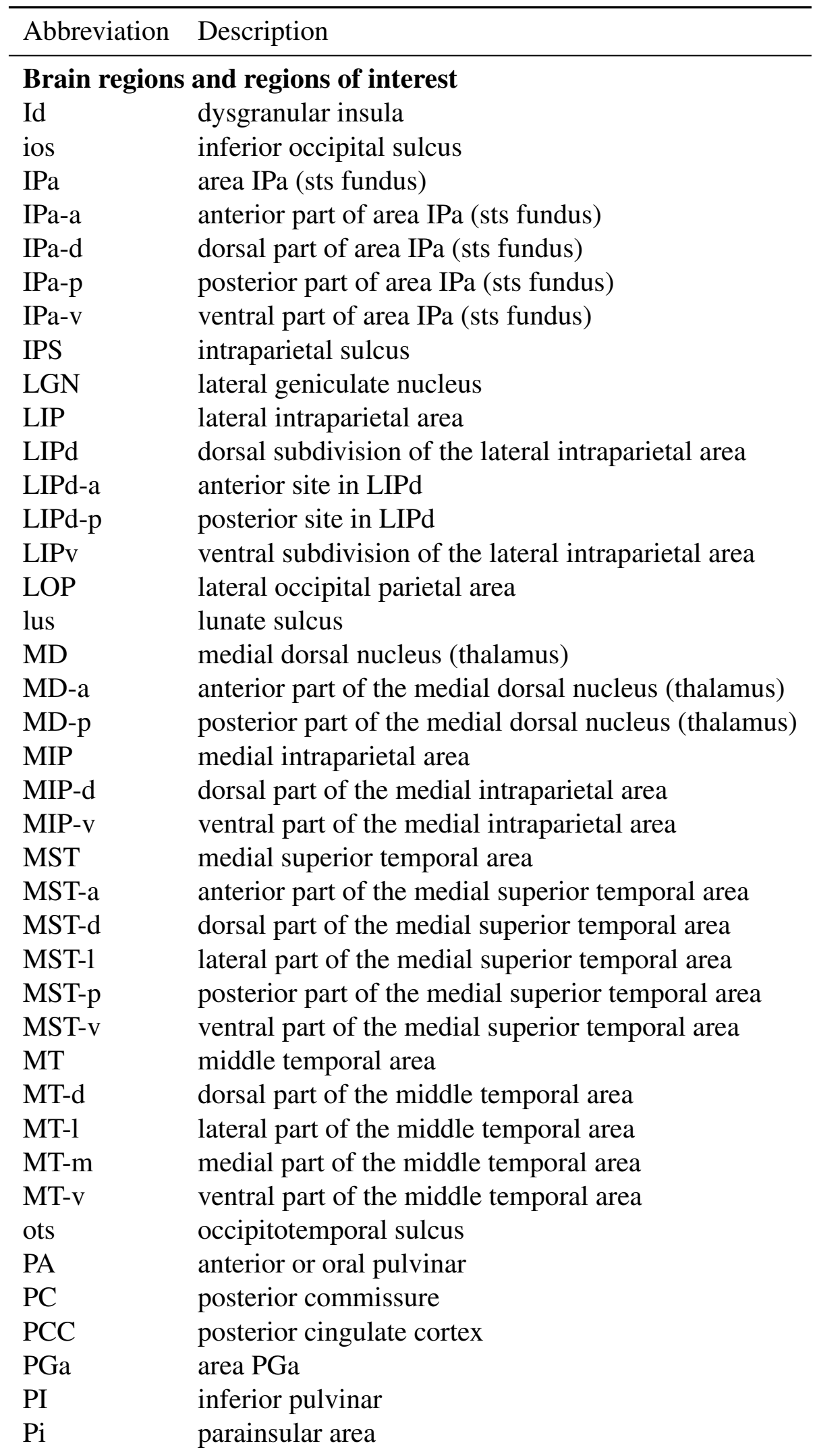




\begin{tabular}{|c|c|}
\hline Abbreviation & Description \\
\hline \multicolumn{2}{|c|}{ Brain regions and regions of interest } \\
\hline PIP & posterior intraparietal area \\
\hline PL & lateral pulvinar \\
\hline PM & medial pulvinar \\
\hline PMd & dorsal premotor cortex \\
\hline pmts & posterior middle temporal sulcus \\
\hline PMv & ventral premotor cortex \\
\hline PO & parieto-occipital area \\
\hline PO-1 & lateral part of the parieto-occipital area \\
\hline PO-m & medial part of the parieto-occipital area \\
\hline pos & parieto-occipital sulcus \\
\hline PPC & posterior parietal cortex \\
\hline ps & principal sulcus \\
\hline RT & rostrotemporal, core region of the auditory cortex \\
\hline $\mathrm{SC}$ & superior colliculus \\
\hline SC-a & anterior part of SC \\
\hline SC-p & posterior part of SC \\
\hline SII & secondary somatosensory area (S2) \\
\hline SMA & supplementary motor area \\
\hline STN & subthalamic nucleus \\
\hline STS/sts & superior temporal sulcus \\
\hline TAa & area TAa (sts dorsal bank) \\
\hline TAa-d & dorsal part of area TAa (sts dorsal bank) \\
\hline TAa-v & ventral part of area TAa (sts dorsal bank) \\
\hline $\mathrm{TE}$ & echo time; area TE (sts ventral bank) \\
\hline TEa & area TEa (sts ventral bank) \\
\hline TEa-d & dorsal subregion of anterior TE \\
\hline TEa-v & ventral subregion of anterior TE \\
\hline TEm & area TEm (sts ventral bank) \\
\hline TEm-d & dorsal part of area TEm (sts ventral bank) \\
\hline TEm-v & ventral part of area TEm (sts ventral bank) \\
\hline TEO & area TEO \\
\hline TEO-d & dorsal part of area TEO \\
\hline TEO-1 & lateral part of area TEO \\
\hline TEO-m & medial part of area TEO \\
\hline TEO-v & ventral part of area TEO \\
\hline TEpd & dorsal subregion of posterior TE \\
\hline TG & temporal pole \\
\hline TG-1 & lateral part of the temporal pole \\
\hline TG-m & medial part of the temporal pole \\
\hline TPO & area TPO (sts, dorsal bank) \\
\hline
\end{tabular}




\begin{tabular}{ll}
\hline Abbreviation & Description \\
\hline Brain regions and regions of interest \\
TPO-a & anterior part of area TPO (sts, dorsal bank) \\
TPO-d & dorsal part of area TPO (sts, dorsal bank) \\
TPO-1 & lateral part of area TPO (sts, dorsal bank) \\
TPO-m & medial part of area TPO (sts, dorsal bank) \\
TPO-p & posterior part of area TPO (sts, dorsal bank) \\
TPO-v & ventral part of area TPO (sts, dorsal bank) \\
Tpt & temporo-parietal area \\
V1 & visual area 1 (primary visual cortex) \\
V1-a & anterior visual area 1, anterior \\
V1-d & dorsal visual area 1 \\
V1-dm & dorsomedial visual area 1 \\
V1-1 & lateral visual area 1 \\
V1-m & medial visual area 1 \\
V1-p & posterior visual area 1 \\
V1-v & ventral visual area 1 \\
V2 & visual area 2 \\
V2-a & dorsal visual area 2 \\
V2-d & dorsal visual area 2 \\
V2-1 & lateral visual area 2 \\
V2-m & medial visual area 2 \\
V2-p & posterior visual area 2 \\
V2-v & ventral visual area 2 \\
V2-vm & ventromedial visual area 2 \\
v23b & area v23b (posterior cingulate cortex) \\
V3 & visual area 3, \\
V3A & visual area V3A \\
V3d & visual area 3, dorsal part \\
V3d-a & anterior visual area 3, dorsal part \\
V3d-1 & lateral visual area 3, dorsal part \\
V3d-m & medial visual area 3, dorsal part \\
V3d-p & posterior visual area 3, dorsal part \\
V3v & visual area 3, ventral part \\
V3v-a & anterior visual area 3, ventral part \\
V3v-p & posterior visual area 3, ventral part \\
V4 & visual area 4 (dorsal part) \\
V4-a & dorsal visual area 4 (dorsal part) \\
V4-d & lateral visual area 4 (dorsal part) \\
V4-1 & V4-p \\
V4t & Vosterior visual area 4 (dorsal part) \\
&
\end{tabular}




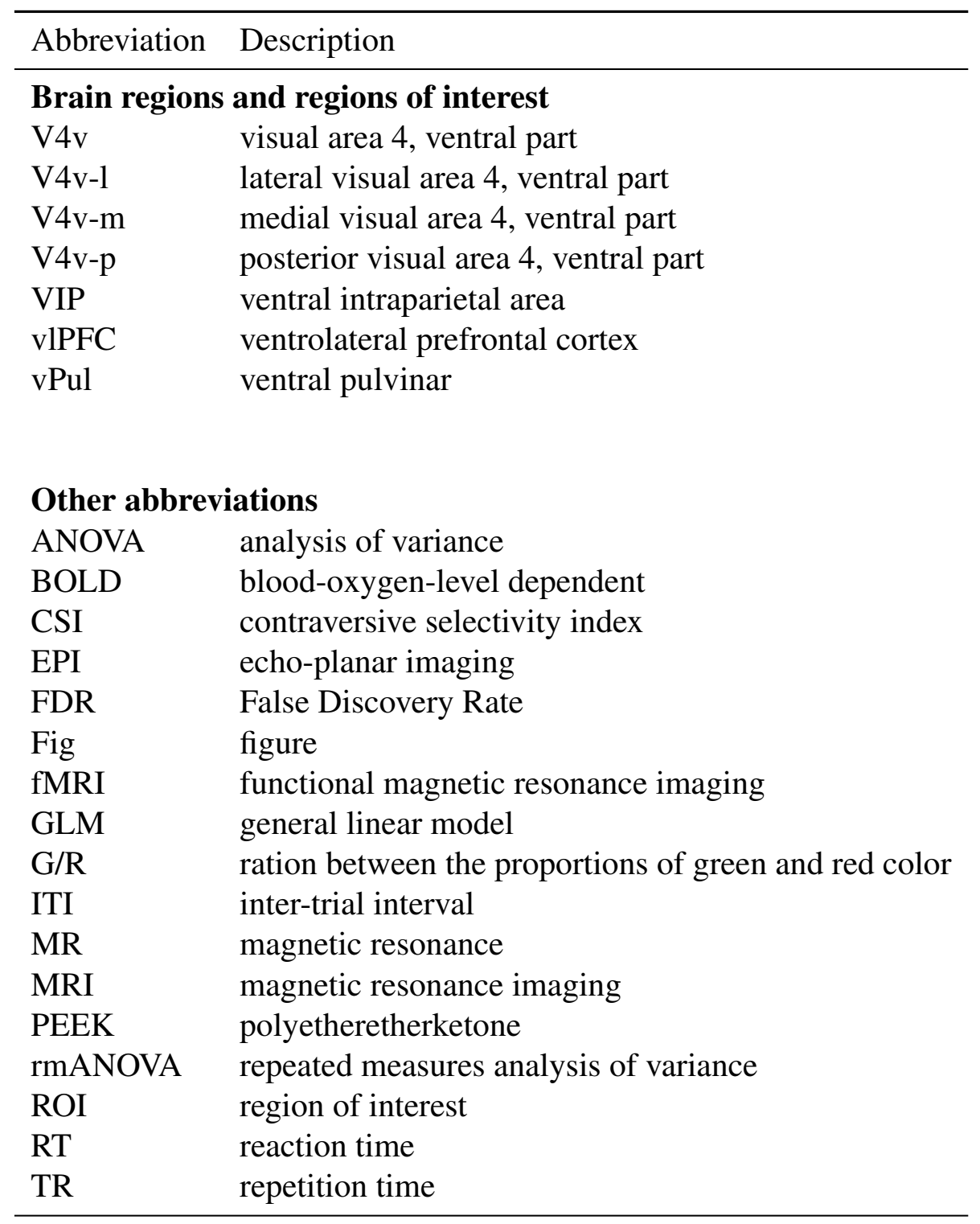





\section{Acknowledgements}

I would like to express my sincere gratitude to my supervisor Dr. Igor Kagan for the continuous support of my doctoral study, for his patience, motivation, and for sharing his knowledge in valuable and fruitful discussions. My sincere thanks also goes to my cosupervisor Prof. Dr. Melanie Wilke for her support and for providing inspiring conceptual input. I also want to thank Prof. Dr. Annekathrin Schacht for insightful comments, valuable advice and for continuous encouragement. I thank all previous and current members of the Cognitive Neuroscience Laboratory for creating such a pleasant and inspiring work environment, for great scientific and intercultural exchange, and for all the fun we had on our business trips. Special thanks go to Lukas Schneider for valuable advice on programming and continuous help with technical issues, Dr. Caio Moreira for the good cooperation in solving issues related to fMRI in behaving monkeys, Ira Panolias and Dr. Daniela Trinca Bertazzi Lazzarini for tremendous technical support, Uwe Zimmermann for his high commitment and friendly personality, and Danial Arabali and Kristin Kaduk for inspiring conversations about science and love. I would also like to thank all members of the Institute of Cognitive Neurology and the Functional Imaging Laboratory for great cooperativeness and valuable technical support, and Curius and Bacchus for being such lovely and cute companions. Last but not least, I want to thank my family, my partner, and close friends for their untiring moral and emotional support in my life and throughout writing this thesis. 



\section{LYDIA GIBSON}

18/03/1989, Ilmenau

Herrenhäuser Str. 108, 30419 Hannover, Germany

lydia.gibson.lg@gmail.com

+4917678019035

\section{EDUCATION AND QUALIFICATIONS}

11/2013 - 12/2017 Doctor rerum naturalium in Systems Neuroscience at Georg-AugustUniversity of Goettingen

Graduation date: 17 December 2017

10/2010 - 11/2012 MSc in Psychology at Georg-August-University of Goettingen

Final mark: 1.0 (very good)

Major subjects: Cognitive Neuroscience, Clinical Psychology, Social

Psychology

10/2007 - 09/2010 BSc in Psychology at Georg-August-University of Goettingen

Final mark: 1.4 (very good)

$2005-2007$

Abitur (equivalent to A level), Goetheschule, Ilmenau

Final grade: 1.4 (very good)

Major subjects: Mathematics, Biology

PROFESSIONAL EXPERIENCE

05/2018-12/2018 Guest researcher, Cognitive Neuroscience Laboratory, Decision and Awareness Group (Dr. Igor Kagan), German Primate Center, Goettingen

11/2013 - 04/2018 PhD student, Cognitive Neuroscience Laboratory, Decision and Awareness Group (Dr. Igor Kagan), German Primate Center, Goettingen

11/2012 - 10/2013 Research assistant, Clinical Neuroscience Group (Prof. Dr. Dr. Hannelore Ehrenreich), Max Planck Institute of Experimental Medicine, Goettingen

04/2009-09/2012 Student assistant at the Department of Experimental Psychology, GeorgAugust-University of Goettingen

HONORS AND AWARDS

$11 / 2012$

Georg-August-University of Goettingen, Master of Science degree awarded "with distinction"

$12 / 2010$

Stipend of the federal state of Lower Saxony, Stipend for outstanding study success 